\title{
TIME
}

\section{TRF1: It Was the Best of Time(s)...}

\author{
Anne Giersch ${ }^{1}$ and Jennifer T. Coull ${ }^{2}$ \\ ${ }^{1}$ INSERM, France \\ ${ }^{2}$ Laboratoire des Neurosciences Cognitives (UMR 7291), Aix-Marseille University \& \\ CNRS, Marseille, France
}

The Timing Research Forum (TRF; http://timingforum.org/) is an international (Fig. 1), gender-inclusive, and open academic society for timing research, founded in 2016 by Argiro Vatakis and Sundeep Teki (Teki, 2016). Following a call to its Committee members in May 2016, we agreed to host the 1st Conference of the Timing Research Forum in Strasbourg, France (TRF1; http://trf-strasbourg.sciencesconf.org). First on the agenda was to decide on eminent keynote speakers to lend credibility to this very first TRF conference. We wanted one talk from the field of psychology and the other from the neurosciences, and so were delighted that both Lera Boroditsky and Warren Meck accepted our invitations immediately. Lera kicked off the conference for us, with an extremely entertaining talk about the spatial representation of time in different societies and cultures and how the linguistic metaphors we use to describe time influence our conception of time. Warren highlighted the key role of the striatal dopaminergic system for timing, illustrating his talk with data from an impressive variety of methodological techniques from the clinical level (performance in patients with Parkinson's Disease) right down to the cellular (optogenetic studies in mice). Coincidentally, the role of dopamine in timing was also the subject of our third keynote talk. One of the mission statements of TRF is to promote the work of young researchers, and Argiro and Sundeep had the great idea to invite an early-career researcher to give a keynote talk. The TRF committee members were invited to submit recommendations of key timing papers published in 2016, and Sofia Soares was the unanimous choice to present her PhD work with Joseph Paton on the timing functions of midbrain dopamine neurons, published in Science (Soares et al., 2016). She 


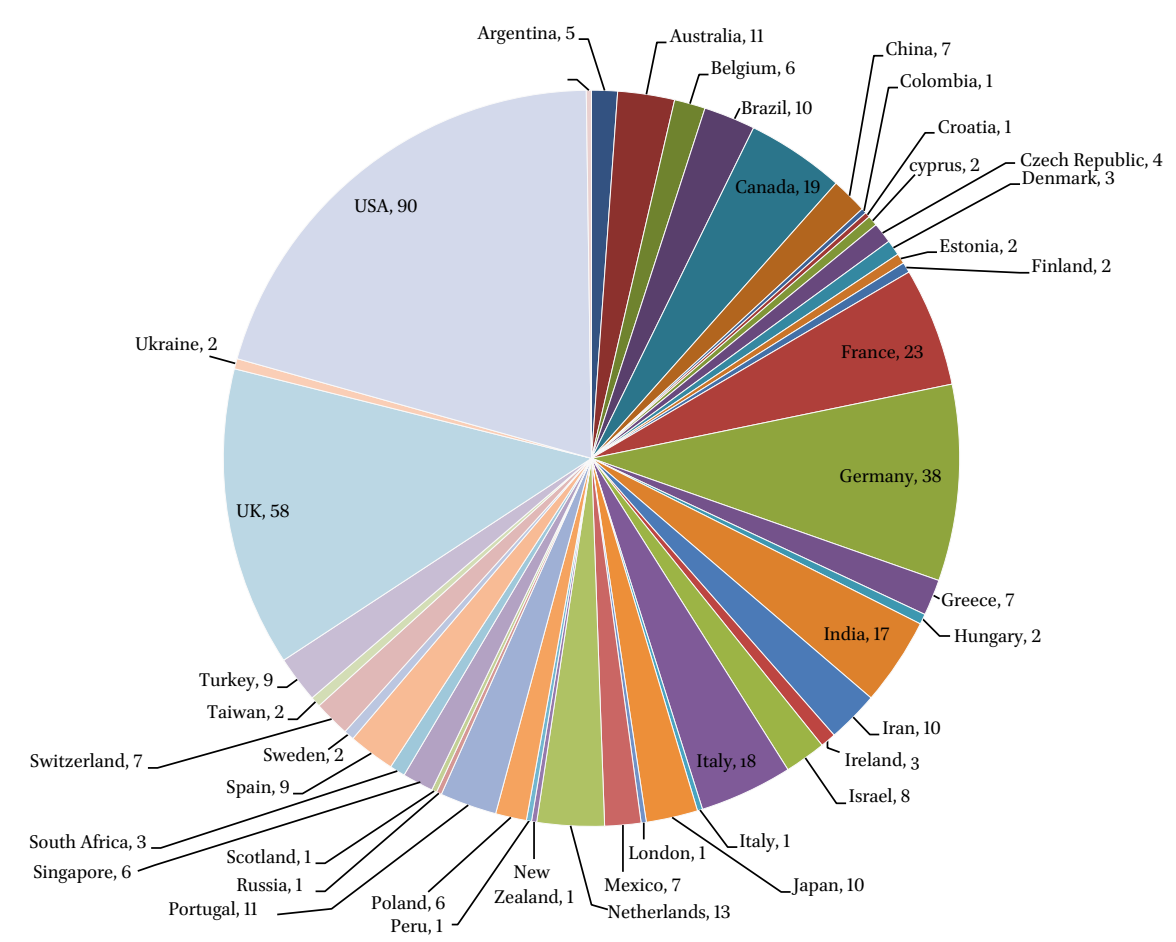

Figure 1. The TRF membership across the world as of October 2017.

delivered an incredibly assured talk, demonstrating that the activity of dopaminergic neurons in the substantia nigra, though not of those in the ventral tegmental area, causally reflects and controls time-based judgments.

We knew TRF had a large membership with more than 650 members and that the timing community was growing. But for a specialized conference, we judged three days of single-track sessions would be sufficient. We therefore planned and announced the dates (October 23-25, 2017) accordingly. However, the incredible response to our call for abstracts $(>200$ submissions) meant that we had far more abstracts than time available. We therefore had to make the difficult decision to program parallel sessions. Some tough scheduling choices would have to be made! We were particularly overwhelmed by the excellent quality of submissions for symposia and wish we could have accepted them all. In the end, we chose a balanced selection of neuroscientific, psychological and computational symposia that spanned timing from the milliseconds range right up to circadian rhythms (see Fig. 2). The 15 symposia (encompassing 52 individual talks, $35 \%$ female presenters) covered a wide spectrum of timing phenomena: how duration is processed (e.g., spatial, motor or embodied representations 


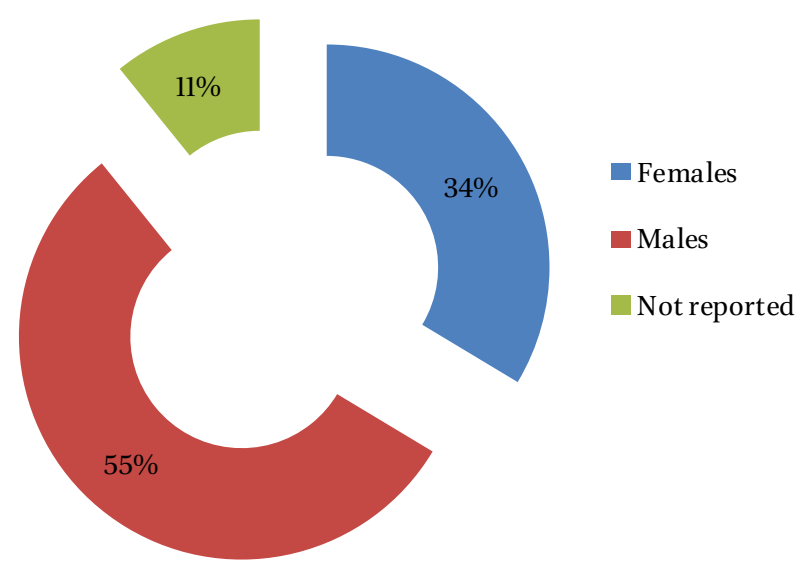

Figure 2. Frequency of time scales associated with TRF1 abstract submissions.

of time; scalar properties of time) and how it can be used for learning or making predictions, how the order of events in time impacts our conscious experience (e.g., intentional binding; the continuous nature of consciousness), and how sensory and neural rhythms guide our sense of time in both humans and animals. Actually, very similar themes were reflected in the submissions for short oral presentations. And once the selected abstracts (36 talks, $42 \%$ female presenters) had been organized into seven themed sessions, the high quality of the presentations made each session actually feel like a premeditated symposium!

We also had a large number of posters (120 posters, $53 \%$ female presenters), which were presented in the beautiful main hall of Strasbourg's UNESCO heritage University Palace over two days. The hall was also the location for the lunch breaks, meaning researchers could gather together and discuss the displayed posters outside of the designated poster sessions. Posters covered an even more diverse range of topics than the talks or symposia. On the first day, we had posters describing how the perception of duration can be modulated by a variety of distinct factors (memory, emotion, motivation, meditation, cognitive effort, salience, visual characteristics, magnitude) and how it is impaired in a number of psychiatric and neurological disorders (e.g., focal lesions, dementia, Parkinson's Disease, schizophrenia, bipolar disorder, depression, ADHD, autism). On the second day, we had neuroscientific investigations of duration processing encompassing several different methodological techniques (electrophysiology, neuroimaging, lesions, pharmacology) and a large number of posters on rhythm processing and temporal predictions. This session also included 


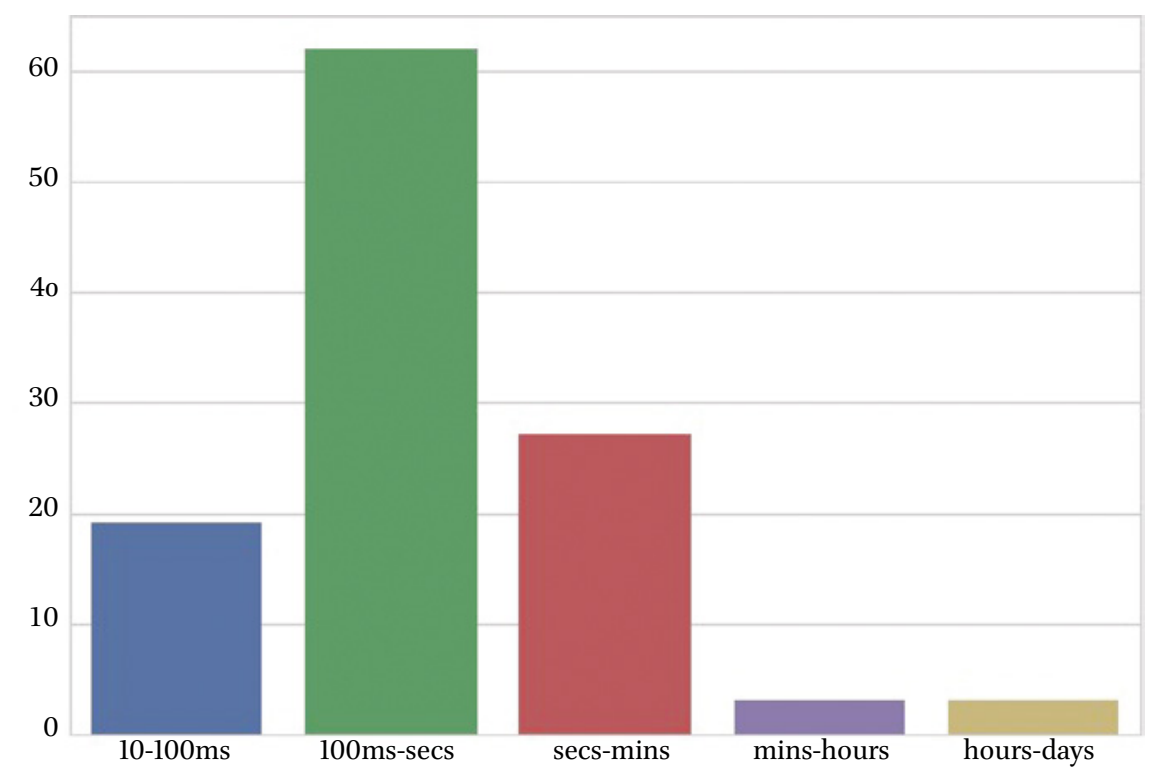

Figure 3. A word cloud featuring keywords from all abstracts presented at TRF1 where the size of a keyword is proportional to its frequency of occurrence across all submissions.

posters on computational models of timing and the role of time perception in computer science and robotics. Finally, we had several posters on multisensory timing, the processing of order and intentional binding. The variety of topics meant there was something for everyone (see Fig. 3), as well as providing the opportunity to discover new ways in which timing can be seen to play a fundamental role in behaviour. Eleven highly rated posters were selected for the poster blitz session, in which junior researchers gave a five-minute talk to publicise their work.

TRF fosters an open research community, evidenced by their collaboration with the Open Science Foundation to host electronic versions of the posters and talks presented at the conference (http://osf.io/view/trf2017). To date, more than 100 submissions have been shared openly with the wider scientific community, demonstrating the commitment of TRF and its members to open science and open access. Additionally, TRF has built a very active online social media presence on Twitter, Facebook, and ResearchGate (more than 1000 followers) and the power of these networks was leveraged before and during the conference to engage with the participants and the wider timing research community. A series of Q\&A interview columns featuring distinguished speakers at the TRF1 conference has been openly shared, highlighting the thoughts and opinions of leading scientists 


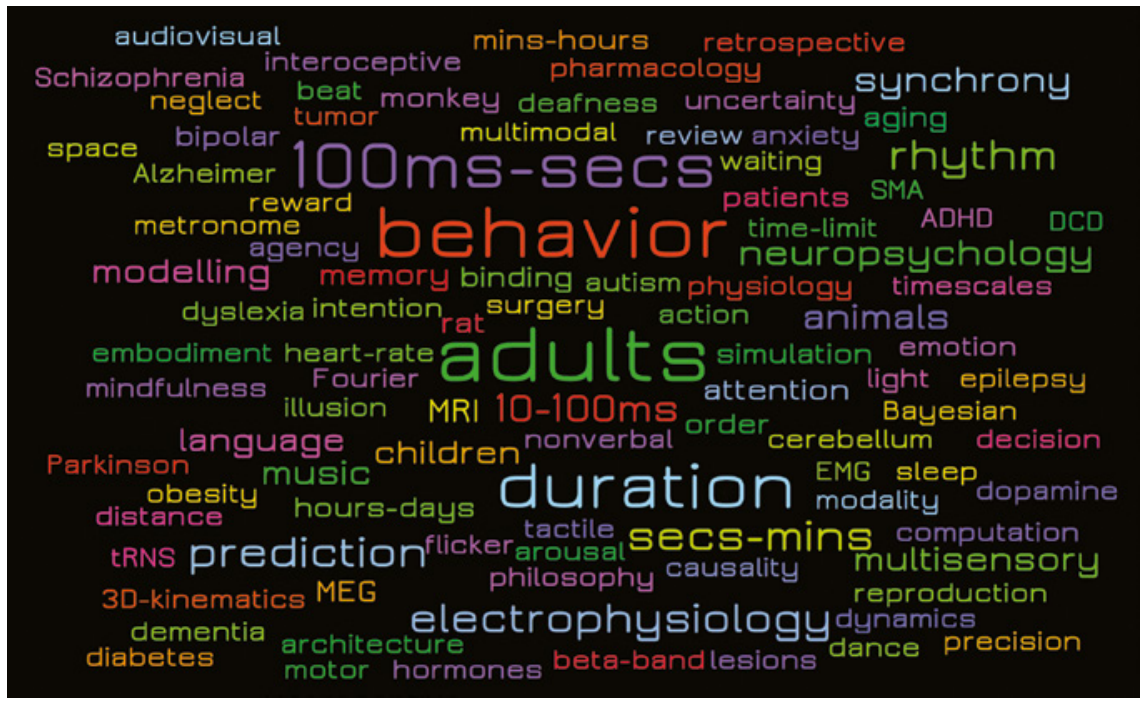

Figure 4. TRF1 participants came to Strasbourg from across the globe. The vast majority $(\sim 70 \%)$ were from Europe with, unsurprisingly, the largest proportion $(30 \%)$ being from the host country France. Other well-represented countries included the UK (11\%), Germany (8\%), USA (7\%), Italy (6\%), the Netherlands (5\%), Japan (5\%) and Brazil (4\%).

on the current state of timing research as well as advice for early-career researchers.

We would like to finish by thanking Argiro and Sundeep, gratefully acknowledging their generosity and dedication in setting up TRF from scratch. Without them, we would not have had the platform that allowed us to bring together 270 people from 28 different countries and five continents (Fig. 4), to describe such diverse aspects of timing research. Thanks to both Sundeep and Argiro for providing a virtual home for the timing research community. But even more importantly, we would like to thank all of the participants for their support and enthusiasm for TRF1. The impressive quality and quantity of the presentations (see this issue), and the stimulating and positive atmosphere during the conference itself, exceeded all our expectations. The huge success of this very first TRF conference attests to the undeniable need for a regular meeting for our international timing community.

\section{References}

Soares, S., Atallah, B. V., \& Paton, J. J. (2016). Midbrain dopamine neurons control judgment of time. Science, 354, 1273-1277.

Teki, S. (2016). A citation-based analysis and review of significant papers on timing and time perception. Front Neurosci, 10, 330. doi: 10.3389/fnins.2016.00330. 


\title{
SYMPOSIA
}

(in order of presentation)

\section{Neural Entrainment as a Mechanism of Efficient Stimulus Processing}

\author{
Benedikt Zoefel' ${ }^{1}$, Alan Archer-Boyd ${ }^{1}$, Matthew H. Davis', \\ Monica N. O'Connell², Annamaria Barczak ${ }^{2}$, Tammy McGinnis ${ }^{2}$, \\ Deborah Ross ${ }^{2}$, Peter Lakatos ${ }^{2}$, Molly J. Henry ${ }^{3}$ and Sanne Ten Oever ${ }^{4}$ \\ ${ }^{1}$ MRC Cognition and Brain Sciences Unit, Cambridge, UK \\ ${ }^{2}$ Nathan Kline Institute for Psychiatric Research, Orangeburg, NY, USA \\ ${ }^{3}$ Brain and Mind Institute, University of Western Ontario, Canada \\ ${ }^{4}$ Department of Cognitive Neuroscience, Faculty of Psychology and Neuroscience, \\ Maastricht University, The Netherlands
}

\section{Symposium organizer: Benedikt Zoefel}

Neural entrainment, the alignment between neural oscillations and rhythmic stimulus input, is often assigned a critical role for stimulus processing, selection, and predictions: By aligning the high-excitability oscillatory phase with the timing of expected events, important stimulus input can be selectively amplified (e.g., Schroeder \& Lakatos, 2009). Nevertheless, studies are often criticized, due to an apparent failure to distinguish neural entrainment from other processes that can potentially produce similar data, or due to a lack of understanding of the underlying neural mechanisms. In this symposium, we use state-of-the-art methods to address these issues in several studies. In the first talk (O'Connell), we demonstrate in intracranial non-human primate recordings that a network comprising non-specific thalamic and cortical regions is critically involved in the phase-reset of neural oscillations, a fundamental mechanism for an efficient adjustment to stimulus input and an often hypothesized process underlying neural entrainment (e.g., Lakatos et al., 2009). In two subsequent talks, we show that neural entrainment is not merely a superposition of regular evoked responses, an issue raised previously (e.g., Capilla et al., 2011): In electroencephalographic (EEG) data (Henry), we show that neural entrainment can be dissociated from evoked responses by independently manipulating the perceived beat and acoustic properties of a rhythmic musical stimulus. In magnetoencephalographic (MEG) recordings (Ten Oever), we show that neural entrainment persists when participants do not consciously perceive the entraining stimulus (which strongly reduces evoked neural activity). In the last talk of the symposium (Zoefel), we use combined transcranial alternating current stimulation (tACS) and functional magnetic resonance imaging (fMRI) recordings to demonstrate that neural entrainment is not only an epiphenomenon but causally involved in speech processing: Manipulating the phase relation between neural oscillations and speech rhythm affects the blood-oxygen-level dependent 
(BOLD) response to intelligible (but not unintelligible) speech. Together, our symposium provides important and complementary evidence that neural entrainment is more than a simple repetition of evoked responses: Instead, it reflects an efficient mechanism for the processing of rhythmic stimulus input, including speech sounds, and involving thalamocortical connections as one of the underlying neural circuitries.

\section{Keywords}

Rhythms, synchrony, electrophysiology, adult, non-human primates, 100s of ms-secs

\section{Mapping the Circuitry of Oscillatory Phase Reset (M. N. O'Connell,}

\section{A. Barczak, T. McGinnis, D. Ross and P. Lakatos)}

To be able to align internal to external rhythms, the brain utilizes a mechanism called phase reset to modulate the phase of neuronal oscillations. It is speculated that inputs originating in the non-specific thalamus are responsible for this mechanism. We tested this hypothesis by recording A1 and MGN neuroelectric activity simultaneously, in awake macaques who were presented with a rhythmic stream of LED flashes. We found that some single neurons in MGN responded to LED flashes, but only on a subset of trials. During trials where the visually-responsive MGN neurons fired, concurrently recorded A1 ensemble activity showed signatures of phase reset, accompanied by significant phase alignment to stimuli. Subsequent to LED related firing in the MGN, a subpopulation of A1 units also fired. Firing of both MGN and Al units was accompanied by stimulus locked high-gamma bursts, indicating likely relevance for the mechanism of phase reset.

\section{Separating Stimulus-Driven and Entrained Neural Responses Using Musical Rhythms (M. J. Henry)}

Interest in the role of neural entrainment in perception of rhythmic environmental stimu$l i$ is on the rise. However, the nature of the neural signals observed in response to rhythmic stimuli is still a matter of debate. Specifically, it is unclear the extent to which these neural responses reflect entrainment of neural oscillations, or may instead reflect superposition of transient responses evoked by stimulus onsets. I will present electroencephalography (EEG) data collected during listening to musical rhythms, where we independently manipulated the strength of the perceived beat and acoustic properties of the rhythms in order to systematically change the energy present in the spectrum of the stimulus envelope at beat- related frequencies. We quantified the contributions of stimulus energy and perceived beat strength to the neural signal. Our results suggest that neural responses to musical rhythms reflect more than a superposition of transient evoked responses, and thus indicate entrainment of neural oscillations.

\section{Temporal Expectations Influence Entrainment Presence and Strength (S. Ten Oever)}

Neuronal oscillations reflect fluctuations in membrane potentials between higher and lower excitable states. It has been proposed that high excitable phases of ongoing oscillations align to rhythmic input streams to optimize sensory processing, so-called entrainment. With MEG recordings we show that entrainment optimizes perception for rhythmic 
streams that are still below perceptual detection thresholds. Interestingly, it was primarily phase alignment, as measured with inter-trial coherence, and not power, that increased prior to detection. These results suggest that the measured entrainment was not solely a consequence of sensory evoked responses and that pre-detection top-down temporal expectations can induce this entrainment. In an EEG study we corroborate on the topdown influences on entrainment by identifying the influence of temporal expectations on entrainment strength. Both studies point to a relevant role of entrainment for behavior and not a mere byproduct of the responses to the sensory input.

\title{
4. Phase Entrainment of Neural Oscillations Is Causally Relevant for Neural Responses to Intelligible Speech (B. Zoefel, A. Archer-Boyd and M. H. Davis)
}

The entrainment of neural oscillations is often considered critical for speech processing. Nevertheless, only if we manipulate entrainment as a dependent variable and observe consequences for speech processing, can we can conclude that there is a causal relation between the two. We manipulated entrainment using transcranial alternating current stimulation (tACS) and systematically varied the phase relation between tACS and an intelligible or unintelligible rhythmic speech stimulus. Recording fMRI data simultaneously, we tested whether this manipulation of entrainment affects neural activity (reflected in the BOLD response). We found that, for intelligible speech, the relation between tACS phase and speech rhythm significantly modulates the magnitude of the BOLD response in the Superior Temporal Gyrus. Importantly, a significant interaction showed that the effect was reduced and absent for unintelligible speech and during sham stimulation. Our results therefore suggest that neural entrainment has a specific, causal influence on neural responses to intelligible speech.

\section{References}

Capilla, A., Pazo-Alvarez, P., Darriba, A., Campo, P., \& Gross, J. (2011). Steady-state visual evoked potentials can be explained by temporal superposition of transient eventrelated responses. PloS One, 6, e14543.

Lakatos, P., O’Connell, M. N., Barczak, A., Mills, A., Javitt, D. C., \& Schroeder, C. E. (2009). The leading sense: supramodal control of neurophysiological context by attention. Neuron, 64, 419-430.

Schroeder, C. E., \& Lakatos, P. (2009). Low-frequency neuronal oscillations as instruments of sensory selection. Trends in Neurosciences, 32, 9-18.

\section{Interrelations Between the Representation of Time and Space}

\author{
Martin Riemer', Esther Kühn', Jonathan Shine', Thomas Wolbers, ${ }^{1,2}$, \\ Roberto Bottini ${ }^{3}$, Baptiste Gauthier ${ }^{4,5}$, Karin Pestke ${ }^{4}$ and Virginie van \\ Wassenhove $^{4}$
}


${ }^{1}$ Aging \& Cognition Research Group, German Center for Neurodegenerative Diseases (DZNE), Magdeburg, Germany

${ }^{2}$ Center for Behavioral Brain Sciences, Magdeburg, Germany

${ }^{3}$ Center for Mind/Brain Sciences, University of Trento, Italy

${ }^{4}$ Cognitive Neuroimaging Unit, CEA DRF/I2BM, INSERM, Université Paris-Sud,

Université Paris-Saclay, NeuroSpin center, F-91191 Gif/Yvette, France

${ }^{5}$ Laboratory of Cognitive Neuroscience, Faculty of Life Science, Brain Mind Institute,

Ecole Polytechnique Federale de Lausanne, Geneva, Switzerland

\section{Symposium organizer: Martin Riemer}

The concepts of space and time are highly intertwined in the human mind. We all are familiar with graphical illustrations of temporal sequences, in which successive events are ordered next to each other along a spatial continuum. The localization of events in time (before/after) and the magnitude of durations (short/long) are often coded with a left-toright spatial order on a 'mental time line' (Bonato et al., 2012). Linguistic approaches point out that we often use spatial metaphors to describe relations in time. The future lies in front of us and past events might be far away. Spatial metaphors for time are accompanied by body gestures, e.g., pointing backwards to indicate that something happened in the past. All these examples demonstrate the general tendency of the human mind to conceptualize in space what is perceived in time (Casasanto \& Boroditsky, 2008). In support of this view, neuroimaging studies have discovered neural populations in the parietal cortex that code both for temporal and spatial magnitudes, and damages in these areas can impair time perception as well as spatial cognition. More recently, the discovery of place and grid cells in the hippocampus and the entorhinal cortex, and the observation that these cells exhibit spatial and temporal tuning curves provide new insights to the neuronal substrates of interrelations between time and space (Kraus et al., 2015). Yet, this wealth of empirical observations is still lacking a coherent theoretical framework that could explain (i) universal and culture-specific aspects of space-time interactions, (ii) whether the cognitive systems for time and space interact in a symmetric or an asymmetric manner, (iii) and whether different forms of space-time interactions (from magnitude judgments to mental time travel) can be traced back to one core neural system or are based on largely different mechanisms. In our symposium, we will present and discuss the results from recent fMRI and MEG/EEG studies, directly comparing neuroanatomical contributions to time and space representations in healthy participants (Riemer). As vision is a pertinent factor for the formation and maintenance of spatial representations, the impact of visual experience of space for time-space interactions will be addressed by comparing sighted and visually impaired participants (Bottini). Interrelations between time and space are not confined to direct perception. They also affect our ability to imagine and mentally represent these dimensions. The second part of our symposium will therefore focus on the question of how mental travel through time is influenced by spatial factors and vice versa. Again, we will discuss the ability for mental travel through time and space in healthy participants (Gauthier). Evaluation of competing hypotheses can produce a common working framework to investigate the links between time and space that are fundamental aspects of human cognition. 


\section{Keywords}

Duration, behavior, MRI, blind patients, neglect patients, secs-mins

\section{Integration Processes of Travel Time and Traveled Distance (M. Riemer, E. Kühn, J. Shine and T. Wolbers)}

The compelling interdependency between the perception of time and space becomes especially evident in the domain of spatial navigation, because moving in space requires time. Knowledge about the length of a covered distance contains valuable information about the corresponding travel time and vice versa. We used fMRI to identify the overlapping and diverging neuronal networks underlying the processing of traveled distance and travel time. In a virtual environment, participants walked along a straight path, while their attention was directed either to travel time or to the distance covered. The results demonstrate lateralization differences in the prefrontal cortex (PFC) for temporal and spatial processing. While attention to travel time is associated with bilateral activation, processing of traveled distance recruits only the right PFC. The presented study extends the research field to the integration of travel time and traveled distance in a navigational context.

\section{The Role of Vision in Space-Time Interactions (R. Bottini)}

Space and time are highly intertwined in the human mind. For instance, things that are longer in space also appear to last longer, and temporal succession is represented along Mental Time Lines that run from left to right or vice versa. It is still unclear how these Spatial-Temporal Associations (STAs) are established, and what is the role of experience in their development. For instance, does the way space and time interact in our mind change across sensory modalities (vision and audition)? Is visual experience necessary to establish space-time conceptual mappings? The study of visually deprived individuals may shed light on these issues. I will present behavioral and psychophysical experiments with sighted and early blind individuals suggesting that, although vision is not strictly necessary to develop STAs, space-time mappings may change as a function of the dominant modality in which we experience the world.

\section{Ordering Events in Time and Space in Mental Travels (B. Gauthier, K. Pestke and V. van Wassenhove)}

How the human brain represents time and space is essential to understand the conscious mind. When moving, mapping the environment yields a topological relationship between the traveled distance and the order of events. Nonetheless, in the absence of movement, e.g., while thinking about future plans, the ordering of mental events may dissociate from their spatial dimension. In this M/EEG neuroimaging study, participants imagined themselves at different times and places (self-projection) and ordered memorized historical events from their mental standpoint. Early parametric changes of evoked responses amplitude, localized in medial temporal region, reflected past-to-future events succession while late spatial ordering evoked components were localized in parietal and frontal cortices. Overall, we report dedicated cortical signatures for the representation of conscious spatial and temporal distance and ordinality in the human brain. Crucially, the directionality of 
the psychological time arrow relies on neural mechanisms that are fundamentally dissociable from spatial directionalities.

\title{
References
}

Bonato, M., Zorzi, M., \& Umilta, C. (2012). When time is space: Evidence for a mental time line. Neurosci. Biobehav. Rev., 36, 2257-2273.

Casasanto, D., \& Boroditsky, L. (2008). Time in the mind: Using space to think about time. Cognition, 106, 579-593.

Kraus, B. J., Brandon, M. P., Robinson, R. J., 2nd, Connerney, M. A., Hasselmo, M. E., \& Eichenbaum, H. (2015). During running in place, grid cells integrate elapsed time and distance run. Neuron, 88, 578-589.

\section{Musical Rhythm: Evolutionary and Cross-Cultural Perspectives}

\author{
Andrea Ravignani ${ }^{12,3}$, Tania Delgado ${ }^{4}$, Simon Kirby ${ }^{5}$, \\ Nori Jacoby ${ }^{6}$, Josh McDermott ${ }^{7}$, Jessica A. Grahn ${ }^{8}$, Daniel J. Cameron ${ }^{6}$, \\ Aniruddh D. Patel ${ }^{9,10}$, Ryuji Takeya ${ }^{11}$, Masashi Kameda ${ }^{11}$ and \\ Masaki Tanaka ${ }^{11}$ \\ ${ }^{1}$ Veterinary and Research Dept., Seal Centre Pieterburen, The Netherlands \\ ${ }^{2}$ Max Planck Institute for Psycholinguistics, The Netherlands \\ ${ }^{3}$ AI-Lab, Vrije Universiteit Brussel, Belgium \\ ${ }^{4}$ Department of Cognitive Science, University of California, San Diego, USA \\ ${ }^{5}$ Centre for Language Evolution, School of Philosophy, Psychology and Language \\ Sciences, University of Edinburgh, UK \\ ${ }^{6}$ The Center for Science and Society, Columbia University, USA \\ ${ }^{7}$ Department of Brain and Cognitive Sciences, Massachusetts Institute of Technology, \\ USA \\ ${ }^{8}$ Brain and Mind Institute and Department of Psychology, Western University, Canada \\ ${ }^{9}$ Department of Psychology, Tufts University, USA \\ ${ }^{10}$ Azrieli Program in Brain, Mind, and Consciousness, Canadian Institute for Advanced \\ Research (CIFAR), Canada \\ ${ }^{11}$ Department of Physiology, Hokkaido University School of Medicine, Japan
}

\section{Symposium organizer: Andrea Ravignani}

Musical rhythm is a ubiquitous feature of the human species (Patel, 2010). Around the world, rich rhythmic and musical structures originate from complex interactions between biological and culturally determined mechanisms (Fitch, 2017). Cross-cultural and cross-species research offer complementary roles in the understanding of the origin of musical rhythm: the former can provide evidence for features that differ from culture to 
culture, whereas the latter point to biologically determined mechanisms that are shared between humans and other animals. In the past few years, paradigms from psychology and music cognition that were previously run predominantly on North American and European subjects have been increasingly incorporated within cross-cultural settings. At the same time, music cognition paradigms have also been increasingly adapted to animal research, providing striking evidence that, given appropriate training, some animals can successfully complete tasks previously considered specific to humans. The crucial bottleneck limiting progress in both fields is the relative lack of paradigms that can be applied in wider experimental contexts (Trehub, 2015). Moreover, three approaches in research on timing have been historically separated. First, the psychology of timing and time perception have been studied in meticulously controlled laboratory experiments. Second, temporal patterns in animal behavior have been investigated by pure behavioral observation, with lack of interest to connect their findings to human cognition. Third, study of the temporal dimension of music, i.e., rhythm, has fallen in the domain of the humanities (classical musicology) and social sciences (cultural anthropology). This symposium showcases work that traverses these domains. Two of the papers describe implementations of a recent experimental technique known as "iterated learning" to rhythm. In iterated learning, participants are asked to reproduce random rhythms; their reproductions are fed back as the stimulus, forming a chain of stimuli that are increasingly dominated by cognitive and productive biases. Ravignani and colleagues use the technique to simulate cultural transmission in the lab, featuring miniature societies of interacting individuals in a controlled environment (and hinting at possible connections with natural animal timing). Jacoby and McDermott use the same technique to extract internal representation of rhythms, showing that the technique can be successfully applied on individuals without musical experience and from a wide range of cultural backgrounds. Grahn and Cameron show how EEG- based music cognition paradigms can be adapted to reveal implicit culturally dependent rhythm processing in individuals from Canada and Africa, while Patel and colleagues show how eye movements can produce predictive and tempo-flexible synchronization in monkeys, behaviors that were recently regarded as human specific. Taken together, these works reveal that challenges and promise of empirically studying the origin of rhythm from both cross-cultural and cross-species perspectives.

\section{Keywords}

Rhythm, music, beat, comparative, cross-cultural, cross-species

\section{Links Predictive and Tempo-Flexible Synchronization to a Visual Metronome in Monkeys (A. D. Patel, R. Takeya, M. Kameda and M. Tanaka)}

Predictive and tempo-flexible synchronization to an auditory beat is a fundamental component of human music. Prior research training macaque monkeys to tap to an auditory or visual metronome has found their movements to be largely reactive rather than predictive. Does this reflect the lack of a capacity for predictive synchronization in monkeys, or a lack of motivation to exhibit this behaviour? To discriminate these possibilities, we trained monkeys to make synchronized eye movements to a visual metronome. We found that 
the monkeys could generate predictive saccades synchronized to periodically alternating visual stimuli when an immediate reward was given for every predictive response. This behaviour generalized to novel untrained tempi, and the monkeys could maintain the tempo internally (i.e., even when the target was triggered by saccades). These results suggest that monkeys have the capacity for predictive synchronization to a visual beat, but are not intrinsically motivated to do it.

\section{Cross-Cultural Comparisons of Neural and Motor Entrainment to the} Beat (J. A. Grahn and D. J. Cameron)

Although all human cultures have rhythm, the rhythmic structures, and perception of those structures, differ across cultures. The current study investigates how culture influences behavioural and neural processing of auditory rhythms. Participants from two cultural groups (San and Hambukushu) from different regions of Botswana, and a group from North America, listened passively to San, Hambukushu, and Western rhythms during encephalographic (EEG) recording. All groups also tapped the beat of the rhythms at a separate time. We predicted that neural and behavioural measures would differ between the cultural groups depending on rhythmic familiarity, with a positive relationship between beat tapping and neural entrainment to particular beat rates. Preliminary analysis suggests cultural differences in both neural and motor entrainment to the beat of musical rhythms, with effects of cultural familiarity present. These novel data provide evidence of how musical rhythm is perceived and entrained to in distinct cultures.

\section{Rhythmic Perceptual Priors Revealed Cross-Culturally by Iterated Reproduction (N. Jacoby and J. McDermott)}

Probability distributions over external states (priors) are essential to the interpretation of sensory signals. We propose a novel method to characterize perceptual priors and apply it to the study of simple rhythms. Simple rhythms have been previously studied using discrimination, categorization or production paradigms. We argue that results obtained using these methods can be re-interpreted as manifesting subjects' reliance on perceptual priors. We developed a method based on iterated reproduction of random temporal sequences. Listeners were asked to reproduce random "seed" rhythms; their reproductions were fed back as the stimulus, and eventually became dominated by internal biases, such that priors could be estimated by applying the procedure multiple times. By testing the paradigm amongst US participants with different levels of musical expertise as well as members of the Tsimane, a native Amazonian society with limited exposure to Western music, we show that priors are strongly modulated by culturally-dependent musical exposure.

\section{The Evolution of Rhythm between Biology and Culture}

\section{(A. Ravignani, T. Delgado and S. Kirby)}

Musical rhythm, beyond its variety, exhibits cross-cultural similarities and statistical universals. Testing the mechanisms underlying these universals, I will show human experiments where musical rhythm is created and evolves culturally due to cognitive and motoric biases. I will also suggest how comparative animal research can help reconstruct early hominid musicality. 


\title{
References
}

Fitch, W. T. (2017). Cultural evolution: Lab-cultured musical universals. Nature Human Behaviour, 1, 0018.

Patel, A. D. (2010). Music, language, and the brain. Oxford: Oxford University Press.

Trehub, S. E. (2015). Cross-cultural convergence of musical features. PNAS, 112, 8809-8810.

\section{Timing and Conditioning: A Contemporary Overview}

\author{
Domhnall Jennings ${ }^{1}$, Charlotte Bonardi ${ }^{2}$, David J. Sanderson ${ }^{3}$, Joseph M. \\ Austen $^{3}$, Rolf Sprengel ${ }^{4}$, and Charles Randy Gallistel ${ }^{\mathbf{5}}$ \\ ${ }^{1}$ CEA1Institute of Neuroscience, Newcastle University, Newcastle upon Tyne, UK \\ ${ }^{2}$ School of Psychology, University of Nottingham, University Park, Nottingham, UK \\ ${ }^{3}$ Department of Psychology, Durham University, Durham, UK \\ ${ }^{4}$ Max-Planck Institute of Medical Research, Department of Molecular Neurobiology, \\ Germany \\ ${ }^{5}$ Rutgers Centre for Cognitive Science, Psych Bldg Addition, Busch Campus, NJ, USA
}

\section{Symposium organizers: Domhnall Jennings and Charlotte Bonardi}

Timing has always occupied a central place in conditioning theory: the conditioning process is engaged by events that are temporally contiguous, and conditioning speed is profoundly affected by temporal factors. Moreover, conditioning tasks result in timing: animals will learn not only that the conditioned stimulus (CS) predicts the unconditioned stimulus (US), but also when that US will occur. But the close relationship between these two phenomena is not mirrored in the theories proposed to explain them. Theories of conditioning and timing are focussed on explaining different aspects of the learned behaviour, often using different behavioural measures. As a consequence most conditioning theories typically offer little explanation of timing, while established timing theories often struggle to account for the broad range of phenomena revealed in conditioning studies (Kirkpatrick, 2014). More recent work has begun to bridge this theoretical chasm, and both timing and conditioning theories have been adapted to encompass a much broader range of effects. In addition more effort has been devoted to exploring the interaction between conditioning and timing manipulations on behaviour. The objective of this symposium is to provide a brief contemporary overview of this research. Bonardi and Jennings' work aims to explore the effect of temporal factors on conditioning, and of conditioning manipulations on timing. Jennings and Bonardi report the results of experiments exploring the effect of latent inhibition training - preexposure of the CS prior to CS-US pairings, which typically retards acquisition of conditioned responding - on timing ability. Their findings suggest that CS preexposure both retards conditioning but enhances timing accuracy. Bonardi and Jennings describe the results of a trace conditioning task, in which a fixed-duration CS is followed by the US after a trace interval which could either be of fixed 
or variable duration. The results suggest that the CS acquired greater associative strength when the trace interval was variable. Sanderson and Austin's presentation focusses on the neural bases of conditioning and timing behaviour, in a report of work with mice lacking the GluAl subunit of the AMPA receptor. These animals fail to show the normal attenuation of learning that is seen with longer-duration CSs. In an elegant series of experiments they demonstrate that this is due to a lack of sensitivity to rate of reinforcement, accompanied by normal sensitivity to number of CS/US pairings. Gallistel's contribution represents the information-processing approach to explaining both conditioning and timing effects. This eschews conditioning theory's usual focus on the trial (a CS-US pairing) in favour of a more global and mathematical analysis of the prevailing environmental conditions. In this presentation Gallistel focusses on the phenomenon of inhibitory learning, in which a CS signals the omission of an otherwise expected US (No-US). The trial-based approach of associative theories struggles to explain events that have not occurred, and Gallistel explores the possibility that this uncertainty might be more fruitfully examined by applying a hazard function to the probability that a reinforcing event will occur.

\section{Keywords}

Conditioning, timing, information, animals

\section{Temporal Control of the Conditioned Response Following CS-Preexposure: Retarded Learning Does Not Mean Retarded Timing (D. Jennings and C. Bonardi)}

When a conditioned stimulus (CS) is paired with an unconditioned stimulus (US), subjects learn not only that the US will be delivered (conditioning), but also when (timing). This learning can be attenuated in some paradigms, such as latent inhibition (LI) in which preexposure of the CS retards learning when CS and US are subsequently paired. However, little is known about the effect of CS preexposure on timing. In a series of experiments using rats in an appetitive procedure, we investigated whether latent inhibition was evident in timing behaviour. Results showed that LI was evident as a lower rate of conditioned responding to the preexposed CS than to a novel cue. Paradoxically, response slopes calculated across the duration of the CS were higher for the preexposed CS: thus, temporal control was better following preexposure. These results indicate that although CS preexposure retards conditioning, it may enhance timing.

\section{Rate-Sensitive Learning Requires the GluA1 Subunit of the AMPA Receptor (D. J. Sanderson, J. M. Austen and R. Sprengel)}

Conditioning occurs more readily with cues of short duration than of long duration. This effect is abolished by deletion of the GluA1 subunit of the AMPA glutamate receptor in mice. The cue duration effect can also be abolished by reinforcing the short duration cue at the same rate as the long duration cue (e.g., a 10 s cue reinforced on $25 \%$ of trials and a 40 s cue reinforced on $100 \%$ of trials), suggesting that sensitivity to reinforcement rate is the cause of the cue duration effect in control mice. GluAl knockout mice are insensitive to reinforcement rate and instead are sensitive to the number of times that a cue is paired with reinforcement, independent of whether rate is manipulated by cue duration 
or probability of reinforcement per trial. These results suggest that GluAl is necessary for weighting numeric information by temporal information in order for rate-sensitivity to be achieved.

\title{
3. The Bernoulli-Gauss and the No-US (C. R. Gallistel)
}

A fundamental problem in the theory of associative learning is the specification of the time at which No-USs "occur". No-USs are events that fail to occur. How can an event that doesn't occur have a time of occurrence? Wilkes and Gallistel propose that the brain represents event times with one of two maximum entropy stochastic models: the exponential and the Bernoulli-Gauss. The Bernoulli-Gauss marries the Bernoulli distribution to the Gaussian distribution to produce a Bernoulli distribution situated in time. Its hazard function provides a natural basis for localizing in time both expected events and expected failures. The hazard for both occurrence and failure to occur rise and subside, demarcating regions in time at which both are anticipated with complementary probabilities.

\section{The Effects of Stimulus Distribution Form on Trace Conditioning (C. Bonardi and D. Jennings)}

Two groups of rats were conditioned to a fixed duration CS in a trace interval task, in which a trace interval separates CS offset and US delivery. For rats in Group Fix the trace interval was of a fixed duration, while for rats in Group Var its duration varied. In contrast to our findings in delay conditioning tasks (where US delivery occurs at CS offset) responding during the CS was higher in Group Var than in Group Fix. However, during the trace interval this difference was reversed - Group Fix responded at a higher rate than Group Var. A further experiment employed a blocking procedure to examine whether the greater response rate observed during the CS in Group Var was due to a performance effect, or the acquisition of greater associative strength by the CS. The results supported the second interpretation. The theoretical implications of these findings will be discussed.

\section{Reference}

Kirkpatrick, K. (2014). Interactions of timing and prediction error learning. Behav. Processes, 101, 135-145.

\section{Time Processing Deficits in Developmental Disorders}

\author{
Laurel J. Trainor ${ }^{1,2,3}$, Andrew Chang', Jennifer Chan', Yao-Chuen $\mathrm{Li}^{4,5}$, \\ John Cairney,,6, B. Tillmann ${ }^{7}$, L.-H. Canette,8,9, N. Bedoin ${ }^{9}$, \\ Usha Goswami $^{10}$, Valdas Noreika ${ }^{11}$ and Christine Falter ${ }^{12}$ \\ ${ }^{1}$ Department of Psychology, Neuroscience and Behaviour, McMaster University, \\ Hamilton, ON, Canada \\ ${ }^{2}$ McMaster Institute for Music and the Mind, McMaster University, Hamilton, ON, \\ Canada \\ ${ }^{3}$ Rotman Research Institute, Baycrest Hospital, Toronto, ON, Canada
}


${ }^{4}$ Department of Kinesiology and Infant and Child Health (INCH) lab, Department of

Family Medicine, McMaster University, Canada

${ }^{5}$ Child Health Research Center, Institute of Population Health Sciences, National Health

Research Institutes, Taiwan

${ }^{6}$ Faculty of Kinesiology \& Physical Education, University of Toronto, Canada

${ }^{7}$ Lyon Neuroscience Research Center CNRS-UMR 5292, INSERM U1028, University Lyon,

France

${ }^{8}$ LEAD-CNRS 5022, Université de Bourgogne, France

${ }^{9}$ Dynamique Du Langage Laboratory CRNS-UMR 5596, University Lyon, France

${ }^{10}$ Centre for Neuroscience in Education, University of Cambridge, UK

${ }^{11}$ Department of Psychology, University of Cambridge, UK

${ }^{12}$ Institute of Medical Psychology, Ludwig-Maximilians-University, Germany

\section{Symposium organizer: Laurel J. Trainor}

Time processing is essential for perception, cognition and action, as well as coordinating between activities and different brain processes. Although most research on the major development disorders, including Dyslexia, Autism, Attention Deficit and Hyperactivity Disorder (ADHD), and Developmental Coordination Disorder (DCD), has focused on unique features of each disorder, there is high comorbidity among them, suggesting that there may be common underlying deficits. One candidate involves deficits in time processing. Studies to date have shown that children with these developmental disorders are more likely to have inferior perceptual timing (e.g., discriminating temporal intervals or rhythms, judging temporal order of events) and/or motor timing (e.g., coordinating actions in time, reproducing auditory rhythms; see Falter \& Noreika, 2014, for a review). However, the commonality of timing deficits across developmental disorders remain far from clear due to the lack of common test paradigms, documented developmental trajectories, and an integrative theory. It appears that Autism involves impaired social timing, Dyslexia involves impaired phonological processing and temporal sequencing of syntax, DCD involves impaired sensorimotor timing, and ADHD involves impaired temporal executive and attentional control. However, the extent to which basic time processing is commonly impaired is not known. The objectives of this symposium are to assess this idea through talks on timing deficits in Dyslexia, Autism, ADHD and DCD that include behavioural and neural measures. The efficacy of timing interventions (e.g., auditory rhythmic cue) in Parkinson's disease and Dyslexia will be discussed, as well as whether such interventions could be successfully applied to other disorders.

\section{Keywords}

Patient, 100s of ms-secs, behaviour, electrophysiology, perceptual and motor timing

\section{Auditory Timing Deficits in Developmental Coordination Disorder (A. Chang, J. Chan, Y.-C. Li, J. Cairney and L. J. Trainor)}

Developmental Coordination Disorder (DCD) is a neurodevelopmental disorder with deficits in motor coordination. Prior research suggests that perceiving auditory timing may involve cortical networks that include motor areas. However, it remains unclear whether 
the motor system is necessary for perceiving time. If this is the case, children with probable DCD should have inferior temporal discrimination performance compared to typically developing (TD) children. In Experiment 1, we investigated discrimination thresholds for auditory unfilled duration and rhythm. The results showed that children with probable DCD have larger thresholds for unfilled duration than TD children, but are not significantly different on rhythm. In Experiment 2, electroencephalogram was recorded while participants listened to auditory oddball sequences with deviations in unfilled duration or rhythm. Analyses on mismatch negativity, which reflects preattentive perceptual encoding, are ongoing. Together, the results suggest auditory and motor systems are connected for perceiving time, and suggest potential auditory interventions for DCD.

\section{Temporal Processing Deficits and Benefits of Rhythmic Auditory Stimulation on Syntax Processing in Developmental Language Disorders (B. Tillmann, L.-H. Canette and N. Bedoin)}

Children with developmental language disorders have been shown to be impaired not only in language processing, but also in rhythm and meter perception. We tested the influence of external rhythmic auditory stimulation (i.e., musical rhythms) on syntax processing in children with specific language impairment and children and adults with dyslexia, using behavioral and electrophysiological measurements. Grammaticality judgments for auditorily presented (correct or incorrect) sentences were better after regular musical prime sequences than after irregular sequences or baseline sequences. In addition, the P600, an electrophysiological marker for processing grammatical errors, was enhanced after regular prime sequences. We also collected data for temporal processing in dyslexic adults. Our findings are interpreted within the Dynamic Attending Theory (Jones, 1976) and the Temporal Sampling (oscillatory) Framework for developmental language disorders (Goswami, 2011). They encourage the use of rhythmic structures (even in non-verbal materials) to boost linguistic structure processing and outline perspectives for rehabilitation.

\section{Reading, Rhythmic Timing and the Brain (U. Goswami)}

Recent neural studies of speech processing provide a temporal "oscillatory" perspective on brain mechanisms for speech encoding. Using these insights, I develop an oscillatory "temporal sampling" neural framework for linking the auditory processing of rhythm to phonological development in dyslexia (Goswami, 2011). I show that for English, sensitivity to temporal rhythmic structure is core to developing good phonological skills, and that English children with dyslexia are relatively insensitive to rhythmic timing. Rhythmic sensitivity is related to how efficiently the brain processes the amplitude modulation (energy) patterns in speech. The rhythmic energy patterns in speech occur at multiple temporal rates simultaneously (delta, theta, beta, gamma). I will describe neural studies showing atypical rhythmic entrainment for dyslexic readers in English.

\section{Time Processing in ASD and ADHD: Shared Deficit or Disorder- Specific Abnormalities? (V. Noreika and C. Falter)}

Individuals with attention-deficit/hyperactivity disorder (ADHD) and autism spectrum disorder (ASD) have been reported to have a range of abnormalities in motor timing, 
perceptual timing, and time perspective functions. However, there is a lack of studies directly comparing time processing between individuals with ADHD, ASD, and typically developing controls. Arguably, three hypotheses should be formally considered when contrasting any two disorders known to have timing abnormalities. A "unification" hypothesis would suggest that ADHD and ASD have a shared timing deficit with similar if not identical timing abnormalities. A "differentiation" hypothesis would propose that ADHD and ASD have disorder-specific timing abnormalities. A "mixed" hypothesis would imply that some of the timing abnormalities are shared between ADHD and ASD, while other timing deficits are disorder-specific. Systematic literature review as well as our own original studies seem to provide preliminary support for the third "mixed" hypothesis.

\section{References}

Allman, M., \& Falter, C. (2015). Abnormal timing and time perception in autism spectrum disorder? A review of the evidence. In A. Vatakis \& M. Allman (Eds.) Time Distortions in Mind-Temporal Processing in Clinical Populations. Leiden: Brill.

Falter, C. M., \& Noreika, V. (2014). Time processing in developmental disorders: A comparative view. In V. Arstila \& D. Loyd (Eds.) Subjective Time: The Philosophy, Psychology, and Neuroscience of Temporality. Cambridge, MA: MIT Press.

Goswami, U. (2011). A temporal sampling framework for developmental dyslexia. Trends Cogn. Sci., 15, 3-10.

\section{Temporal Organization of Perceptual Processes by Motor-Driven Low-Frequency Neuronal Oscillations}

\section{Benjamin Morillon ${ }^{1}$, Alice Tomassini ${ }^{2}$, Pieter Medendorp ${ }^{2}$, Eric Maris ${ }^{2}$ and Daniele Schon ${ }^{1}$}

${ }^{1}$ Aix Marseille Univ, Inserm, INS, Institut de Neurosciences des Systèmes, Marseille, France

${ }^{2}$ Donders Institute for Brain, Cognition and Behavior, The Netherlands

${ }^{3}$ Auditory Language Group, Department of Basic Neuroscience, University of Geneva, Switzerland

\section{Symposium organizer: Benjamin Morillon}

A consistent body of research in the last decade has highlighted the role of oscillatory activity in sensory processing, leading to the notion that perception is inherently discrete and periodic. More recently, the focus has also turned to the role of motor processes in the proposed periodic nature of perception. This goes far beyond the long- standing idea that externally-triggered movements might be dominated by rhythmic components. The motor system might actually be capable of exerting endogenous control of oscillatory activity that entails perceptual consequences. Motor-related modulations of perceptually-relevant 
oscillations have been shown when sensory information is predictable in time - suggesting that the motor system orchestrates the temporal tuning of attention, optimizing information extraction during active exploration (Morillon et al., 2014; Arnal et al., 2015). Furthermore, oscillations in perceptual performance time-locked to the execution of voluntary movements have been shown for stimuli that are unpredictable and, importantly, unrelated to the motor task, pointing to a rather automatic form of sensory-motor coupling (Tomassini et al., 2015). In this symposium, we will present novel findings that reveal a leading role of the motor system in the temporal prediction of external events and the modulation of sensory processing through rhythmic brain activity, and discuss their implications for action-perception coupling mechanisms.

\section{Keywords}

Prediction, rhythms, behavior, MEG, adult, 100s of ms-secs

\section{Theta Oscillations Synchronize Perception with Motor Intention}

\section{(A. Tomassini, P. Medendorp and E. Maris)}

Our motor system orchestrates the sampling of sensory information by orienting our receptor organs in space and time. Mounting evidence further suggests that motor signals also contribute to the actual analysis of the incoming sensory data, thereby shaping the perceptual outcome. I will present behavioral and neurophysiological findings showing that the motor system can modulate low-level sensory function, even when the movement does not involve the receptor organ and the stimuli are irrelevant for the motor performance. Crucially, this motor-related modulation has a rhythmic signature, occurs in an anticipatory fashion - i.e., during movement planning - and entails perceptual consequences. We found rhythmic fluctuations of visual perception, which are time-locked to the execution of voluntary hand movements and emerge before movement onset. This movement-locked rhythmicity in perceptual performance is predicted by the phase of EEG theta oscillations $(\sim 4 \mathrm{~Hz})$ very early during movement planning $(>1$ s before movement). Moreover, theta oscillations are phase- locked to the onset of the movement. Remarkably, the alignment of theta phase and its perceptual relevance unfold with similar non-monotonic profiles, indicating their relatedness. Action planning hence seems to be accompanied by an endogenous phase adjustment of perceptually-relevant brain oscillations. The present work suggests that action and perception may be bound in an automatic way since the very early processing stages through neuronal oscillatory activity in the theta range.

\section{Motor Origin of Temporal Predictions in Auditory Attention (B. Morillon)}

Temporal predictions are fundamental instruments for facilitating sensory selection, allowing humans to exploit regularities in the world. It is proposed that the motor system instantiates predictive timing mechanisms, helping to synchronize temporal fluctuations of attention with the timing of events in a task-relevant stream. I will present a neurophysiological account for this theory in a paradigm where participants track a slow reference beat while extracting auditory target tones delivered on-beat and interleaved with 
distractors. At the behavioral level I will show that overt rhythmic movements sharpen the temporal selection of auditory stimuli, thereby improving performance. Capitalizing on magnetoencephalography recordings I will provide evidence that temporal predictions are reflected in Beta-band $(\sim 20 \mathrm{~Hz})$ energy fluctuations in sensorimotor cortex and modulate the encoding of auditory information in bilateral auditory and fronto-parietal regions. Together, these findings are compatible with Active Sensing theories, which emphasize the prominent role of motor activity in sensory processing.

\title{
3. Music to Speech Entrainment (D. Schon)
}

The Dynamic Attending theory suggests that attention is entrained to the rhythmic structure of music via stimulus brain oscillatory coupling. Thus, different point in time will be differently attended. Indeed, stimuli presented at attended points in time, corresponding to the strong metrical beats of music, are processed faster and more accurately compared to stimuli presented out of beat or on weak beats. This holds true when the target stimulus is presented in the visual modality. Importantly this effect last after the rhythmic stimulus end, showing a carry-over effect of entrainment in time. Recent work demonstrates that a rhythmic stimulus can facilitate the processing a of a following speech stimulus. I will provide evidence of the mechanism underlying this music to speech coupling as well as the functional relevance in terms of speech processing and speech rehabilitation.

\section{References}

Arnal, L. H., Doelling, K. B., \& Poeppel, D. (2015). Delta-beta coupled oscillations underlie temporal prediction accuracy. Cerebral Cortex, 25, 3077-3085.

Morillon, B., Schroeder, C. E., \& Wyart, V. (2014). Motor contributions to the temporal precision of auditory attention. Nat. Comm., 5, 5255.

Tomassini, A., Spinelli, D., Jacono, M., Sandini, G., \& Morrone, M. C. (2015). Rhythmic oscillations of visual contrast sensitivity synchronized with action. J. Neurosci., 35, 7019-7029.

\section{Neural Oscillations for Time Estimation}

\author{
Martin Wiener', Nandakumar Narayanan ${ }^{2}$, Tadeusz W. Kononowicz ${ }^{3}$, \\ Clemence Roger ${ }^{3}$, Virginie van Wassenhove ${ }^{3}$, Alomi Parikh ${ }^{4}$, Arielle \\ Krakow ${ }^{5}$ and H. Branch Coslett ${ }^{4}$ \\ ${ }^{1}$ George Mason University, USA \\ ${ }^{2}$ University of Iowa, USA \\ ${ }^{3} \mathrm{CEA} / \mathrm{DRF} /$ NeuroSpin, INSERM Cognitive Neuroimaging Unit, France \\ ${ }^{4}$ University of Pennsylvania, USA \\ 5Johns Hopkins University, USA
}

Symposium organizer: Martin Wiener 
The perception of time and prediction of upcoming events requires coordination between a diverse set of neural regions. Further, interval timing may be fractionated into separate yet overlapping neural circuits that are invoked for different temporal contexts across the brain (i.e., rhythmic vs. non-rhythmic). Neural oscillations have emerged as a candidate mechanism for neural timing and the coordination of activity across different timing contexts and brain regions (Wiener \& Kanai, 2016). Across the assemblage of frequency bands, numerous associations have been made between distinct frequency bands and timing functions in particular task contexts. For example, delta oscillations (1-4 Hz) have been observed coordinating frontal and cerebellar circuits during interval timing (Parker et al., 2017), whereas beta oscillations (15-25 Hz) have recently been associated with supra-second temporal reproduction (Kononowicz \& van Rijn, 2015). In this symposium, we will provide an overview of various candidate oscillations and speculate on their precise function for interval timing. The work presented in this symposium will span both human and animal models, and include a variety of methods to provide convergent findings.

\section{Keywords}

Oscillations, perceptual timing, noninvasive brain stimulation, decision making, accumulation

\section{Delta Oscillations and the Starting Gun (N. Narayanan)}

Timing processes can be initiated by cues. However, how these cues engage neuronal timing mechanisms is unclear. Here, we present evidence that during fixed-interval timing, cues trigger delta- frequency $(1-4 \mathrm{~Hz})$ oscillations in medial frontal cortex of humans and rodents, These rhythms require medial frontal D1 dopamine receptors. We show that this rhythm can engage single neurons in the rodent striatum and subthalamic nucleus and the human subthalamic nucleus that are involved in timing. Furthermore, stimulating medial frontal D1 dopamine-receptor expressing neurons at delta frequencies can compensate for depleted frontal dopamine. These data indicate that D1-dependent delta oscillations may play a role in initiating the neural mechanisms controlling timing.

\section{Temporal Metacognition as Decoding Self-Generated Brain Dynamics (T. W. Kononowicz, C. Roger and V. van Wassenhove)}

In The awareness of one's sources of errors and misjudgments is key to cognitive and behavioral improvement. People can self-assess various types of cognitive performance when decisions involve external perception. However, without external feedback, how can internal timing and errors be at once generated by the same brain? Strikingly, we show that humans' ability to generate a duration and to self-assess their timing rely on an internal variable set by the synchronization of beta oscillations. Using time-resolved neuroimaging, we show that the strength of beta synchronization informs on how distant from a set internal variable one's time production is. Beta synchronization sets a ballistic trajectory for time production, which is read-out and integrated in the performance monitoring subsystems as alpha desynchronization following the production of duration, enabling awareness of timing errors. Altogether, our study provides novel insights on how the human brain may decode purely internal dynamics during temporal metacognition. 


\title{
3. Causal Role of Beta Oscillations in Time Estimation (M. Wiener,
}

\section{A. Parikh, A. Krakow and H. B. Coslett)}

Recent evidence has suggested that beta oscillations $(15-25 \mathrm{~Hz})$ serve as a supramodal index of timing functions in the brain. Yet, no study has investigated whether beta oscillations are causally related to timing functions. To provide a comprehensive overview of beta's involvement in timing, we first re-analyzed two prior EEG datasets (Wiener et al., 2012; Wiener \& Thompson, 2015) from perceptual timing paradigms, and observed changes in beta associated with the encoding of durations into memory. Next, we tested 20 human subjects with transcranial alternating current stimulation (tACS) over the supplementary motor area at alpha $(10 \mathrm{~Hz})$ and beta $(20 \mathrm{~Hz})$ frequencies during a temporal bisection task, and found that beta stimulation exclusively shifts the bisection point, while preserving precision. Finally, we decomposed behavioral data with a drift diffusion model, finding that the shift in perception can be tied to a change in the starting point of evidence accumulation.

\section{References}

Kononowicz, T. W., \& van Rijn, H. (2015). Single trial beta oscillations index time estimation. Neuropsychologia, 75, 381-389.

Parker, K. L., Kim, Y. C., Kelley, R. M., Nessler, A. J., Chen, K.-H., Muller-Ewald, V. A., Andreasen N. C., \& Narayanan, N. S. (2017). Delta-frequency stimulation of cerebellar projections can compensate for schizophrenia-related medial frontal dysfunction. Mol. Psychiatry., 22, 647-655.

Wiener, M., \& Kanai, R. (2016). Frequency tuning for temporal perception and prediction. Current Opinion in Behavioral Sciences, 8, 1-6.

\section{Timing, Neural Dynamics, and Temporal Scaling}

\author{
Dean Buonomano ${ }^{1}$, Hugo Merchant ${ }^{2}$ and Mehrdad Jazayeri $^{3}$ \\ ${ }^{1}$ Departments of Neurobiology and Psychology, UCLA, USA \\ ${ }^{2}$ Laboratorio B-15, Instituto de Neurobiología - UNAM, Querétaro, México \\ ${ }^{3}$ McGovern Institute, Robert A. Swanson Career Development Professor in the Life \\ Sciences, Brain and Cognitive Sciences MIT, USA
}

\section{Symposium organizer: Dean Buonomano}

There is increasing experimental and theoretical support for the notion that timing on the scale of hundreds of milliseconds to second relies on neural dynamics-i.e., changing populations of neural activity, in which time is encoded in the population of neurons active at any given movement (population clocks). Electrophysiological recordings in a large number of brain areas, including the motor cortex, premotor cortex, prefrontal cortex, hippocampus, and striatum have reported that it is possible to decode elapsed time from 
reproducible patterns of neural activity in these areas. A number of questions, however, remain unclear, including the neural mechanisms underlying temporal scaling, that is, the ability to produce the same motor patterns at different speeds. This symposium will explore behavioral and neurophysiological data, and neurocomputational models, underlying timing and temporal scaling in the range of hundreds of milliseconds to seconds.

\section{Keywords}

Timing, neural dynamics, neurophysiology, neurocomputation

\section{Periodic Neural State Trajectories Underlie Rhythmic Tapping (H. Merchant)}

The ability to generate rhythms of different tempos is a hallmark of higher cognition. We know that tapping to a regular beat engages neurons from the medial premotor cortices (MPC). Yet, the neuronal population code behind rhythmic tapping remains elusive. Here we found that the activity of hundreds of primate MPC neurons show a strong periodic pattern that becomes evident when its activity is projected into a lower dimensional state space. We show that different tempos are encoded by circular trajectories of different radii and that this neuronal code is highly resilient to the number of participating neurons. Crucially, the oscillatory dynamics in neuronal state space is a signature of cognitive timing under metronome guidance or when is internally controlled, and is not the result of repetitive motor commands. Our results support the notion that rhythmic behaviors are encoded by the dynamic state of MPC neural populations.

\section{Flexible Temporal Control of Self-Initiated Movements by Speed of Cortical Dynamics (M. Jazayeri)}

The medial frontal cortex plays a fundamental role in temporal control of movements and has been implicated in inhibition, initiation, and coordination of voluntary actions. However, the underlying computational principles and neural mechanisms remain largely unknown. We recorded from the medial frontal cortex of monkeys trained to flexibly produce different intervals with different effectors. Neurons displayed complex and heterogeneous activity patterns. Despite this complexity, response profiles of many neurons were similar when scaled to a reference interval. This property indicates that the produced interval can be understood in terms of the speed of response modulations across the population along invariant neural trajectories. A recurrent neural network emulating this behavior revealed that (1) speed is regulated by an external input exploiting single-neuron nonlinearities, and (2) invariant trajectories are governed by recurrent interactions controlling dynamics. Together, these findings reveal the coding principles and potential mechanisms by which cortical dynamics flexibly control self- timed voluntary movements.

\section{Temporal Scaling of Complex Temporal Patterns in a Recurrent Neural Network Model and Humans (D. Buonomano)}

Timing is fundamental to complex motor behaviors: from tying a knot to playing the piano. A general feature of motor timing is temporal scaling: the ability to produce similar motor patterns at different speeds. Here we report that temporal scaling is not an intrinsic property of motor timing: after learning to tap a Morse code signal subjects were not 
able to accurately generate it at faster and slower speeds. We then demonstrate that while temporal scaling is also not a general property of recurrent neural networks (RNNs), that RNNs can be trained to produce robust temporal scaling. The model captures a signature of motor timing-Weber's law-but predicts that timing will be more precise at faster speeds. This prediction was confirmed in a temporal production task: the standard deviation of a response at the same absolute time varied across speeds.

\title{
Perception: Continuous or Discrete?
}

\author{
Michael Herzog ${ }^{1}$, Adrien Doerig ${ }^{1}$, Rufin VanRullen² ${ }^{2}$ Tomer Fekete ${ }^{3}$ \\ and Cees van Leeuwen ${ }^{3}$ \\ ${ }^{1}$ EPFL, Switzerland \\ ${ }^{2}$ CerCo, CNRS, Universite de Toulouse, France \\ ${ }^{3}$ KU Leuven, Belgium
}

\section{Symposium organizers: Michael Herzog and Adrien Doerig}

Intuitively, consciousness seems to be a continuous stream of percepts. A diver is jumping off a cliff. We see her trajectory against the blue sky at each single moment in time. Many models in vision rely explicitly and implicitly on continuous perception. For example, in visual backward masking, continuous perception is assumed to explain how a trailing mask can render a preceding target unconscious. However, continuous accounts are in fact challenged by demonstrations where, as in masking, a trailing element determines perception. Another classic example is the color phi paradigm. When a green disk is presented for a short time, it is perceived as such. When a second red disk is presented a bit later and at a different location, motion is perceived during which the color changes midway. Perception has changed qualitatively. Whereas discrete models have been proposed for centuries, continuous perception seems to be so intuitive that there are only a few explicit proponents. In this symposium Cees van Leeuwen and Tomer Fekete (Leuven) will propose and defend the continuous account against discrete proposals. Rufin VanRullen (Toulouse) will show how brain rhythms produce discrete perception, which can be envisioned as a one-stage model of discrete perception. Michael Herzog and Adrien Doerig (Lausanne) will propose a two-stage model of discrete perception, in which the temporal resolution of unconscious processing is independent of the duration of percepts, which can last up to $400 \mathrm{~ms}$. Clearly, the temporal nature of perception is one of the fundamental questions in perception research and, as mentioned, the answer to this question has important ramifications for most fields of perception science. For this reason, it is surprising to see this topic remain largely unexplored. This controversial symposium is the first step in understanding the temporal nature of perception in a systematic fashion.

\section{Keywords}

Consciousness, resolution, modeling, adult, 10s-100s of ms 


\section{Perceptual Cycles (R. VanRullen)}

Brain function involves oscillations at various frequencies. This could imply that perception and cognition operate periodically, as a succession of cycles mirroring the underlying oscillations. This age-old notion of discrete perception has resurfaced in recent years, fueled by advances in neuroscientific techniques. Contrary to earlier views of discrete perception as a unitary sampling rhythm, contemporary evidence points not to one but several rhythms of perception that may depend on sensory modality, task, stimulus properties, or brain regions. In vision for example, a sensory alpha rhythm $(\sim 10 \mathrm{~Hz})$ may co-exist with at least one more rhythm performing attentional sampling around $7 \mathrm{~Hz}$. Although these timescales are clearly slower than the temporal resolution observed for many visual features (e.g., 30-40Hz for motion or flicker), there is no contradiction as long as discrete sampling takes place after these features have been extracted from the sensory input stream.

\section{Two Steps: Quasi-Continuous Unconscious Processing and Discrete Conscious Perception (M. Herzog and A. Doerig)}

Perception seems to be a continuous stream and, for this reason, we implicitly assume that perception is continuous. However, we need to integrate information across time. For example, in apparent motion we do not perceive first a static disk and then another static disk but a smooth trajectory, favoring discrete accounts of perception. What is the sampling rate of discrete perception? Usually, the sampling rate is determined by temporal resolution. If we cannot perceive two flashes of light presented $40 \mathrm{~ms}$ after each other, discrete sampling cannot be faster than $40 \mathrm{~ms}$. However, different paradigms have shown evidence for sampling rates ranging from 3 to $300 \mathrm{~ms}$. Here, we propose that the sampling rate in these paradigms is determined by the temporal resolution of unconscious processing, which is independent of the changes of conscious percepts, occurring at a much lower rate, likely in the range of $400 \mathrm{~ms}$, as supported by TMS experiments.

\section{For the Sake of Maintaining Continuity (T. Fekete and C. van Leeuwen)}

For perception to be discrete, neuronal networks must switch states fast enough for us to see, e.g., objects moving. Our data suggest that even for local brain networks this already would render discrete switching practically indistinguishable from continuous progression. In fact discrete switching faces even higher speed demands when inter area communication is considered, given neuronal signaling delays, resulting in asynchrony and enforced waiting times. Brains cannot support discrete switching at these rates. Moreover, postulating it turns out to offer no explanatory advantage, not even for the data claimed to support it. Our experience largely appears continuous, and where rapid transitions occur, as in perceptual switching or sensorimotor coordination, the underlying processes are complex and involve long-term dependencies that accumulate gradually. As a theoretical construct, discreetness therefore offers no added value over continuity. In contrast, continuous multiscale dynamics suffers no such drawbacks and naturally accommodates experience as we know it. 


\title{
Temporal Binding of Actions to Their Effects: Underlying Mechanisms and Implications for Cognition, Perception, and Development
}

\author{
Marc J. Buehner', Teresa McCormack ${ }^{2,3}$, Sara Lorimer ${ }^{2,3}$, \\ Emma Blakey ${ }^{2,3}$ and Christoph Hoerl ${ }^{4}$ \\ ${ }^{1}$ Cardiff University, School of Psychology, Park Place, Cardiff, UK \\ ${ }^{2}$ University of Sheffield, Western Bank, Sheffield, UK \\ ${ }^{3}$ Queens University Belfast, University Road, Belfast, Northern Ireland, UK \\ ${ }^{4}$ University of Warwick, Department of Philosophy, Coventry, UK
}

\section{Symposium organizers: Marc Buehner and Teresa McCormack}

Temporal binding (TB) refers to the mutual attraction in subjective time between a cause and its effect: Relative to single-event baseline judgments, people's perception of causal actions and their outcomes systematically shifts in subjective awareness. Specifically, causal actions are perceived relatively later, while their outcomes are perceived relatively earlier - action and outcome attract each other in subjective experience (Haggard et al., 2002). However, TB has also been reported when time perception is probed directly: Verbal estimates and reproductions of intervals between causal actions and their outcomes are reliably shorter than those of control intervals (Humphreys \& Buehner, 2010), and psychophysical measures of causal vs non-causal intervals reveal shorter PSEs in the former than the latter case (Nolden et al., 2012). Because the majority of demonstrations of TB deployed intentional action as the critical cause, TB is now increasingly deployed as a convenient proxy measure for sense of agency. Moreover, reduced TB for negative outcomes has been interpreted to reflect reduced sense of agency for actions that bring about negative consequences: For example, reduced TB for penalties delivered to a peer when following an experimenter's instruction (as opposed to under free will) is taken to show that coercion reduces one's sense of agency for self-action (Caspar et al., 2016). The robustness and widespread use of TB notwithstanding, the structural underpinnings and wider implications of TB are still only poorly understood. Very little is known about whether it originates from changes to interval timing, event perception, or both, whether it follows from cue-combination or constraint-satisfaction processes, and how it relates to other cognitive processes. This symposium brings together perspectives from experimental and developmental psychology and philosophy to shine light on the mechanisms underlying TB as well as its implications in a wider context, and its usefulness as a proxy for less tangible constructs such as sense of agency, and causal belief.

\section{Keywords}

Duration, behaviour, adults, children, 100s of ms-secs, attentional modulation, temporal binding 


\section{Temporal Binding and Internal Clocks (M. J. Buehner)}

Most accounts of TB interpret it as stemming from delayed awareness of causal actions combined with earlier awareness of their effects - an approach that can simultaneously explain the two different manifestations of TB reported in the literature: shifts in action perception and changes to interval timing. Changes to time perception, however, are traditionally explained in light of internal clock models (e.g., Gibbon et al., 1984), either as modulations of pacemaker speed (clock rate), or timing latency. I will present evidence from a series of studies showing that TB is associated with reduced clock speed during causal intervals. Moreover, this modulation is specific to the cause-effect interval and does not affect the timing of other, concurrent events. These results are difficult to reconcile with cue combination or belief-updating approaches to TB and question whether TB arising from prospective timing preparations is subserved by the same principles as TB arising from event perception or action preparation paradigms.

\section{When Causality Shapes the Experience of Time (E. Blakey and S. Lorimer)}

Two studies examined the developmental origins of TB. Participants of all ages (6- to 10year olds and adults) reported that a causal interval between a key-press and an on-screen rocket launch felt shorter than that same interval between a predictive signal and launch. In a stimulus anticipation task, 4- to 11-year olds and adults predicted when an on-screen event would occur. Participants of all ages predicted that the event would occur earlier on trials where they were aware of a causal mechanism leading to the event compared to trials were the event was merely signaled by a predictor. Notably, TB decreased with age. These results demonstrate that children's temporal experience, like that of adults, is affected by causal representations. The results point to a bidirectional relation between time and causality that exists early in development and persists into adulthood, and may be privileged very early in development.

\section{Temporal Binding and the Perception/Cognition Boundary (C. Hoerl)}

Temporal experience has become a particular vibrant area of debate in recent philosophy of mind. TB presents a challenge to one model of temporal experience put forward in this context - the view that we can perceive temporal extension and structure simply because our experiences, too, unfold through time and share the same temporal extension and structure. In response, a defender of this view might argue that TB reflects an error in judgment rather than perception (see, e.g., Phillips, 2014, for related arguments). This raises the general issue as to where to draw the boundary between perception and cognition. I will argue that research on $\mathrm{TB}$ - specifically on the mechanisms underpinning it - can in fact also help to show how questions about the perception/cognition boundary should be adjudicated in the context of temporal experience.

\section{References}

Caspar, E. A., Christensen, J. F., Cleeremans, A., \& Haggard, P. (2016). Coercion changes the sense of agency in the human brain. Curr. Biol., 26, 1-16. 
Gibbon, J., Church, R. M., \& Meck, W. H. (1984). Scalar timing in memory. Ann. N. Y. Acad. Sci., 423, 52-77.

Haggard, P., Clark, S., \& Kalogeras, J. (2002). Voluntary action and conscious awareness. Nat. Neurosci., 5, 382-385.

Humphreys, G. R., \& Buehner, M. J. (2010). Temporal binding of action and effect in interval reproduction. Exp. Brain Res., 203, 465-470.

Nolden, S., Haering, C., \& Kiesel, A. (2012). Assessing intentional binding with the method of constant stimuli. Conscious. Cogn., 21, 1176-1185.

Phillips, I. (2014). Experience of and in time. Philos. Compass, 9, 131-144.

\title{
Temporal Prediction: Dynamics in Single Neurons and Networks
}

\author{
Matthew S. Matell ${ }^{1}$, Krystal L. Parker ${ }^{2}$, Joseph J. Paton ${ }^{3}$, Valérie Doyère ${ }^{4}$, \\ and Dean V. Buonomano ${ }^{5}$ \\ ${ }^{1}$ Department of Psychology, Villanova University, USA \\ ${ }^{2}$ Department of Psychiatry, University of Iowa Carver College of Medicine, USA \\ ${ }^{3}$ Champalimaud Centre for the Unknown, Portugal \\ ${ }^{4}$ Paris-Saclay Institute of Neuroscience (NeuroPSI), CNRS-Université Paris-Sud, Orsay, \\ France \\ ${ }^{5}$ Departments of Neurobiology and Psychology, University of California, Los Angeles,
} USA

\section{Symposium organizer: Matthew S. Matell}

While we have had a good understanding of the primary behavioral characteristics of temporal control for several decades (Gibbon, 1977), the psychological and neural mechanisms that give rise to this critical facet of behavior remain unclear. Indeed, there are a number of different classes of models, including accumulation and decay models, state-dependent network models using oscillators or intrinsic neural dynamics, and sequential-behavior models that all provide excellent accounts of the behavioral data. Similarly, the neural structures that might underlie these processes span the range of dedicated structures such as the striatum and cerebellum, to the intrinsic dynamics of small and large networks that can be exhibited throughout the brain (Ivry \& Schlerf, 2008). Deciphering the mechanisms that underlie timing will therefore require investigating and perturbing the activity patterns of neurons in behaving animals (Merchant et al., 2013). This symposium will provide a forum in which four internationally renowned experts will discuss their work investigating neural activity within different brain areas and how their data support a particular framework for understanding temporal perception and control. Krystal Parker (University of Iowa) will provide evidence that frontal and cerebellar networks interact with one another to facilitate temporal control, and that dysfunction in these circuits contributes to 
schizophrenia-related temporal disorganization. Joseph Paton (Champalimaud Centre for the Unknown) will present activity patterns of the striatum and its midbrain dopaminergic input neurons and will discuss the direct involvement of these neurons in temporal prediction and control. Valérie Doyère (Paris-Saclay Institute of Neuroscience) will discuss time-related alterations in coupled neural activity patterns in the amygdala and striatum as subjects learn to predict the time of an aversive event. Finally, Dean Buonomano (University of California Los Angeles) will argue that the criticality of temporal prediction for adaptive behavior requires mechanisms that can exist throughout the nervous system. Together, this symposium will address whether network size and specialization is critical for temporal prediction, and whether these factors vary as a function of the duration and behavior in question.

\section{An Essential Role for the Cerebellum in Suprasecond Timing}

\section{(K. L. Parker)}

Timing is a fundamental cognitive process, which relies on the frontal cortex. Timing is impaired in schizophrenia, which involves abnormalities in the medial frontal cortex. Here we investigate a novel strategy to normalize medial frontal brain activity by stimulating cerebellar projections. We used an interval timing task requiring frontal and cerebellar networks that are disrupted in schizophrenic patients. We report three novel findings. First, patients with schizophrenia had dysfunctional delta rhythms between $1-4 \mathrm{~Hz}$ in the medial frontal cortex. Second, we found in animal models that both frontal and cerebellar neurons were modulated during interval timing and had delta-frequency interactions. Finally, delta-frequency optogenetic stimulation of thalamic terminals on lateral cerebellar projection neurons rescued timing performance as well as medial frontal activity in a rodent model of schizophrenia-related frontal dysfunction. These data provide insight into how the cerebellum influences medial frontal networks and the role of the cerebellum in cognitive processing.

\section{Basal Banglia Contributions to a Time-Based Decision (J. J. Paton)}

Time is a fundamental dimension of experience, critical for extracting meaning from the environment and constructing behavior. However, the neural mechanisms for timing are poorly understood. We trained rodents to judge time intervals as longer or shorter than 1.5 seconds while recording and manipulating activity of neurons the dorsal striatum and dopamine neurons in the substantia nigra pars compacta. Time was encoded by population dynamics of striatal neurons in a manner that predicted duration judgments, striatal muscimol infusions degraded performance, and cooling striatal tissue led to underestimation of interval duration, suggesting that striatal dynamics underlie animals' timing behavior. Using fiber photometry, we found that higher/lower dopaminergic activity predicted under/overestimation of interval duration. Surprisingly, optogenetic activation/suppression caused under-/over-estimation of interval duration. These data suggest that interactions between dopamine neurons and striatal networks can cause variability in timing, with broad implications for reinforcement based decision-making. 


\title{
3. An Amygdala-Striatal Network for Temporal Expectation of an Aversive Stimulus (V. Doyère)
}

In Pavlovian aversive conditioning, the subject not only learns an association between a neutral conditioned stimulus (CS) and an aversive unconditioned stimulus (US), but also that the CS predicts the time of arrival of the US. I will present a series of experiments using local field potential recordings in awake rats during auditory aversive conditioning, which show dynamical neural activities between the dorsomedial striatum and the basolateral amygdala as a correlate of CS-US interval processing. Results show that these structures belong to a common functional network during the temporal expectancy of the US arrival, and that adaptation to new temporal contingencies involves plasticity in the striatum under amygdala control. I will discuss how this network may participate in the encoding of the CS-US interval over the course of learning, in parallel to the development of behavioral temporal control, at least in Pavlovian aversive conditioning in a supra-seconds range.

\section{Timing Is an Intrinsic Computation of Neural Circuits (D. V. Buonomano)}

Time is at the core of the brain's main functions: 1) the brain stores information about the past in order to allow animals to predict and prepare for the future- the degree to which animals succeed in predicting the future translates into the evolutionary currency of survival and reproduction; 2) The brain tells time across scales ranging over 12 orders of magnitude because timing is not only critical for decoding sensory information and producing motor responses, but for prediction - the circadian clock is in sense a prediction device, it predicts when the sun will rise. Because time is so fundamental to brain function we argue that timing is highly distributed process, one that is embedded at the deepest levels into the brain's hardware. In other words, most neural circuits are able to tell time and process temporal information in one form or another.

\section{References}

Gibbon, J. (1977). Scalar expectancy theory and Weber's law in animal timing. Psychol. Rev., 84, 279-325.

Ivry, R. B. \& Schlerf, J. E. (2008). Dedicated and intrinsic models of time perception. Trends Cogn. Sci., 12, 273-280.

Merchant, H., Harrington, D. L., \& Meck, W.H. (2013). Neural basis of the perception and estimation of time. Annu. Rev. Neurosci., 36, 313-336.

\section{Listen to Your Heart: Our Inner Perception and Experience of Time}

\author{
Nicola Cellini' ${ }^{1}$, Giovanna Mioni ${ }^{1}$, Marc Wittmann², \\ Alexandre C. Fernandes ${ }^{3}$, Teresa Garcia-Marques ${ }^{3}$ and Olga Pollatos ${ }^{4}$ \\ ${ }^{1}$ Department of General Psychology, University of Padova, Padova, Italy
}




\section{${ }^{2}$ Institute for Frontier Areas in Psychology and Mental Health, Freiburg, Germany ${ }^{3}$ ISPA - Instituto Universitário William James Center of Research ${ }^{4}$ Clinical and Health Psychology, Institute of Psychology and Education, Ulm University, Germany}

\section{Symposium organizers: Nicola Cellini and Giovanna Mioni}

Recent studies suggest a key role of bodily signals in the cognitive processing of time. Indeed, it has been showed that basal physiology as well as arousing situations, such as changes in body temperature, skin conductance level, muscle reactivity, and cardiac activity may affect our perception of time. For example, studies have showed how subjective experience of time is lengthened when physiological arousal increases as results of presentation of emotional stimuli, stressing situations, or mood variations. Based on these results, it has been proposed that physiological changes may not just represent the output of a possible pacemaker interfering with subjective time, but they can work as timekeepers themselves. It has been also proposed that time perception may be an embodied property of our cognition, which relies on affective and interoceptive states, that are dependent on internal bodily signals. In other words, the subjective temporal experience of external events relies on physiological rhythms, which are then integrated by cortical networks responsible for integrating bodily signals (Wittmann, 2013). This suggests that the experience of time is the result of a combination of visceral integration (i.e., interoceptive awareness) and autonomic (sympathetic/parasympathetic) control and it is based on the temporal integration of afferent signals from the body itself (Craig, 2002). Although these fascinating models aim to integrate classical cognitive models with physiological indices, the studies supporting them are showing inconsistent results, mainly due to experimental and methodological challenges. In this symposium, we aim to describe the state of art of the field and we aim to open a discussion about how body and mind are working together to keep the clock ticking and to track the passage of time, and to try to overcome the challenge this field is facing.

\section{Keywords}

Heart rate variability, interoceptive processes, embodiment, electromyography, physiology

\section{Waiting through Time: How the Bodily Self Shapes the Experience of Time (M. Wittmann)}

Based on conceptual considerations in neuroscience and phenomenology intertwined affective and interoceptive states create the experience of time. Subjective time emerges especially in waiting situations with longer duration. A series of studies with time intervals in the range of several minutes were conducted and which were filled with different situational contexts: (1) individuals with higher scores in impulsivity and present-fatalistic time orientation relatively overestimate duration; (2) watching a staged professional dance, spectators who pay more attention to the own body signals feel that time is relatively slowed down; (3) after body-centered meditation, individuals experience waiting in a more relaxed way, time to pass faster, and they are less future oriented; (4) effects on 
the timing of perceived stimuli right after meditation are influenced by heart rate variability recorded during meditation. Subjective time thereafter is modulated through timerelated personality factors and variations in states of the embodied self during waiting.

\section{Cardiac Activity Modulates Temporal Perception (N. Cellini and G. Mioni)}

Recent studies have shown that physiological signals (i.e., skin conductance) may predict and modulate our precision in temporal tasks. In two studies, we aimed to explore the relationship between temporal abilities and autonomic activity at rest and after a stressful situation. In the first study participants were asked to perform a time-bisection and two finger tapping tasks. In the second study, students were tested in the same time bisection task after either performing a stressing attentional task or the non-stressing version of the task. Prior to these tasks, we measured several electrophysiological indices at rests and during performance. Our results showed that increased heart rate variability at baseline was associated with higher temporal accuracy. Also, after the stressful situation, which increases heart rate, participants showed a more accurate and less variable temporal performance. Our results are consistent with the idea that bodily signals may shape our perception of time.

\section{Spontaneous Facial Muscle Activity Predicts Duration Estimation (A. C. Fernandes and T. Garcia-Marques)}

In this paper we address the hypothesis that temporal dynamics of spontaneous muscle activity may index the subjective representation of objective duration. Neural motor system (SMA) was already suggested to be involved in building-up (ramping activity) a representation of duration in explicit perceptual timing tasks. Also sustained attention, critical to time perception, was suggested to be associated with electromyographic-gradients activation varying proportionally to non-motor task's duration. We test our hypothesis on a perceptual timing task where familiar and non-familiar targets were presented in different durations, monitoring two facial-muscles' activity: the zygomaticus-major (supposedly indexing familiarity positivity) and the corrugator-supercilii (supposedly indexing attention). Data corroborate our hypothesis showing spontaneous electromyographic activity of each muscle to reflect different timing perception processes. Whereas the ramping corrugator activity (its duration and amplitude) reflected objective-duration as being a significant predictor of subjective duration estimates, the zygomatic overall activity predicted familiarity (a non-temporal factor) subjective duration bias.

\section{Perception of Time and Body Awareness (O. Pollatos)}

Internal signals like one's heartbeats are centrally processed via specific pathways and both their neural representations as well as their conscious perception (interoception) provide key information for many cognitive processes. Recent research suggest that bodily systems like the cardiovascular system might be specifically associated with this ability, while few studies have assessed this relationship in more detail and therefore should be presented here with the focus on interoceptive signal processing. Two main tasks (retrospective time 
estimation, time interval reproduction) were used, while either interoceptive focus was manipulated or heartbeat information and interoceptive accuracy were assessed. Main result suggests that heart cycle and time reproduction measures responses are associated for certain time interval lengths. Information obtained from the cardiac cycle is relevant for the encoding and reproduction of time in the time span of 2 to 25 seconds. Sympathovagal tone as well as interoceptive processes mediate the accuracy of time estimation. Retrospective temporal distortions are directly influenced by attention to bodily responses. Sympathetic nervous system activation affecting memory build-up might be the decisive factor influencing retrospective time judgments, highlighting the relevance of interoception for the effects of emotional states on subjective time experience.

\section{References}

Craig, A. D. (2002). How do you feel? Interoception: The sense of the physiological condition of the body. Nat. Rev. Neurosci., 3, 655-666.

Wittmann, M. (2013). The inner sense of time: How the brain creates a representation of duration. Nat. Rev. Neurosci., 14, 217-223.

\section{Circadian Rhythms in Health and Disease}

\section{Etienne Challet ${ }^{1}$, Claude Gronfier ${ }^{2}$, Fabien Pifferi ${ }^{3}$, and Valérie Simonneaux ${ }^{3}$}

${ }^{1}$ Regulation of Circadian Clocks Team, Institute of Cellular and Integrative

Neurosciences, CNRS and University of Strasbourg, France

${ }^{2}$ Inserm U1208, Stem Cell and Brain Research Institute, Univ Lyon, Bron, France

${ }^{3}$ UMR CNRS MNHN MECADEV - Adaptative Mecanisms and Evolution 1, Brunoy, France

\section{Symposium organizer: Valérie Simonneaux}

All biological functions display daily rhythms, which are synchronized to the environment and among each other by a network of primary and secondary biological clocks. This symposium will discuss the mechanisms underlying the daily regulation of essential functions like sleep, locomotor activity and feeding through the use of unconventional animal models. It will also report how desychronization of daily rhythms has negative impacts on health and ageing processes.

\section{Circadian Rhythms and Metabolism (E. Challet)}

Most biological functions, including metabolism, display circadian rhythms. This temporal order is controlled by a network of circadian clocks, comprising a master clock in the hypothathamic suprachiasmatic nuclei, mainly reset by ambient light, and secondary clocks in the brain and peripheral tissues, synchronized by the master clock and mealtime. Calorie restriction and diet-induced obesity differentially modify the suprachiasmatic clockwork. 
Metabolic pathologies are frequently associated with circadian disorders. Conversely, epidemiological studies indicate that chronic shiftwork is highly correlated with increased incidence of type 2 diabetes, obesity, and cardiovascular disease. Furthermore, circadian desynchronization induced by chronic jetlag in rodents negatively impacts metabolism by triggering increased adiposity and impaired glucose tolerance. Chronic jetlag also leads to a shortening of telomeres, a biological marker of cellular aging. In conclusion; there are reciprocal interactions between metabolism and circadian clocks. Metabolic diseases induce circadian disturbances while circadian desynchronization is a potential cause for the metabolic syndrome.

\section{Sleep and Biological Rhythms: It's a Matter of Time! (C. Gronfier)}

Sleep remains a mystery for most of us. It is critical for life since it is shared across all animal species: fishes, birds, mammals, and even worms and insects. The structure of sleep, its duration and its timing during the day are different across species, and even within species. Some are diurnal and sleep at night, others are nocturnal or crepuscular. Some are long sleepers, others are short sleepers. The same is found in humans. Within a same individual, sleep changes with age, from birth to death, in its structure, duration, and timing. The circadian biological timing system is the central master clock driving these phenomena. Sleep is a matter of time. The biological clock is the time keeper that orchestrates the physiological functions involved across the body. Sometimes, either due to internal or external factors, the symphony becomes cacophony, sleep is disturbed, the clock desynchronized, and health is compromised.

\section{Is Biological Clock at the Core of Aging Process? Contributions from a Photoperiodic Non-Human Primate (F. Pifferi)}

The ability of organisms to adapt to their environment during aging is altered. Age-related disorders in Human include disturbances of biological rhythms, especially sleep-wake rhythms alterations, and perturbations of body temperature and hormone secretion. The alteration of biological rhythms with age leads to major health consequences, particularly due to the alteration of sleep-wake rhythms that causes a strong alteration of the general condition. The study of these changes is therefore a major health issue and requires the use of appropriate animal model such as the grey mouse lemur (Microcebus murinus), a small Malagasy primate with very pronounced biological rhythms. Studies from this species brought useful information on the role of biological rhythms (both circadian and seasonal) and biological clock in health and longevity. On the one hand, results on the relationship between aging and the clock, illustrated by rhythms alteration in elderly individuals will be presented. On the other hand, the demonstration that the clock, through the alternation of seasonal cycles, may be key process in aging will be discussed. A final illustration of the major role played by the biological clock in the aging process is provided by the circadian resonance theory, which suggests that the closer the endogenous period of an individual or species is to $24 \mathrm{~h}$, the longer is its longevity. First physiological exploratory data of the underlying mechanisms of this theory in mouse lemur will also be presented. 


\title{
Embodied Timing: The Role of Emergent and Predictive Timing Mechanisms in the Voluntary Control of Whole Body Movements
}

\author{
Yvonne Delevoye-Turrell ${ }^{1}$, Daniel Lewkowicz ${ }^{1}$, Juliane J. Honisch ${ }^{2}$, \\ Mark T. Elliott ${ }^{3}$, Nori Jacoby ${ }^{4}$, Alan M. Wing ${ }^{5}$, Pieter-Jan Maes ${ }^{6}$, \\ Valerio Lorenzoni ${ }^{6}$, Joren $\mathrm{Six}^{6}$, Simone Dalla Bella ${ }^{7,8,9}$, \\ Valérie Cochen de Cock $^{10,11}$, Dobromir Dotov ${ }^{7,12}$, Sophie Bayard ${ }^{13}$, \\ Christian Geny ${ }^{11}$, Petra Ihalainen ${ }^{7}$ and Benoît Bardy ${ }^{13,8}$
}

ISCALab - UMR CNRS 9193, Room. A4.319, Building A, Domaine du Pont de Bois, 59653 Villeneuve d'Ascq, France

${ }^{2}$ School of Psychology and Clinical Language Sciences, University of Reading, Reading, RG6 6AL, UK

${ }^{3}$ Institute of Digital Healthcare, University of Warwick, Coventry, CV4 7AL, UK

${ }^{4}$ Department of Brain and Cognitive Sciences, Massachusetts Institute of Technology, Cambridge, Massachusetts, USA

${ }^{5}$ School of Psychology, University of Birmingham, Edgbaston, B15 2TT, UK

${ }^{6}$ IPEM, Dept. of Art, Music and Theatre Sciences, Ghent University, Ghent, Belgium

${ }^{7}$ EuroMov Laboratory, Montpellier University, Montpellier, France

${ }^{8}$ Institut Universitaire de France (IUF), Paris, France

${ }^{9}$ International Laboratory for Brain, Music, and Sound Research (BRAMS), Montreal, Canada

${ }^{10}$ Beau Soleil Clinic, Montpellier, France

${ }^{11}$ Centre Hospitalier Universitaire de Montpellier, Montpellier, France

${ }^{12}$ Centro de Ciencias de la Complejidad, Universidad Autónoma de México, Brasil

${ }^{13}$ Department of Psychology, Paul Valery University, Montpellier, France

\section{Symposium organizer: Yvonne Delevoye-Turrell}

Intentional motor behaviour requires the planning of body actions through space and time. Depending on the nature of the task, the goal can be geared towards the needs to produce actions at a specific moment in time. For example, clapping to a beat requires a person to set the focus on the time intervals between successive arm strokes to perform a given rhythmic pattern. In other cases, timing is simply an emergent property that reveals itself when producing cyclic movements through space. Limb velocities contain inherent dynamics that will pace motor actions at a given tempo. Such phenomenon can be observed in such cases as waving good-bye, knocking at the door or going out for a Sunday jog. The study of motor timing was initiated by Fraisse and collaborators back in the 60's using a very simple finger tapping task that provided the means to reveal not only the natural spontaneous tempo of body movement but also the ability of a person to synchronise motor elements to an external metronome. Concerned by the emergent properties of motor timing, other studies developed the use of repetitive wrist/arm flexions to study 
the emergence of rhythmic coupling. This important literature has suggested through the years that predictive timing and emergent timing modes of motor control may in fact be related to different neurobiological mechanisms. Depending on the temporal constraints set upon the task, one or other of the timing modes may be implicated for motor planning and execution. Nevertheless, such suggestions are based solely on the use of simple one joint action patterns of an individual's performance. The objective of the present symposium is to report studies focused on the timing properties of motor behaviour in whole body movement by exploring timing strategies within solo and group scenarios. This symposium will present four studies in which whole body 3D kinematics were used to reveal temporal patterns of motor control when interacting in an emotional contained environment. Overall, we aim to show how 3D kinematics can provide a way to confirm the complementary role of the automatic emergent and the cognitively controlled time measurements for optimized intentional motor behaviour. Data from healthy participants and patients with movement disorders will be presented.

\section{Keywords}

3D kinematics, interval timing, synchrony, music, emotion, behaviour, neuropsychology, time series analysis, adult, Parkinson, 100s of ms-secs, secs-mins

\section{Cue Properties Change Timing Strategies in Group Synchronisation (J. J. Honisch, M. T. Elliott, N. Jacoby, and A. M. Wing)}

Research suggests that individuals optimise their timing performance by minimising variability of timing errors between external cues and their own movements. However, at the cost of increasing the timing variability of their own movements. The present study investigated whether an individual's timing strategy changes according to the task, in a group scenario. We employed a novel paradigm that positioned six individuals to form two chains with common origin and termination on the circumference of a circle. We found that participants with access to timing cues from only one member used a strategy to minimise their asynchrony variance. In contrast, the participant at the common termination of the two chains, who was required to integrate timing cues from two members, used a strategy that minimised movement variability. We conclude that humans are able to flexibly switch timekeeping strategies to maintain task demands and thus optimise the temporal performance of their movements.

\section{When your Movements Betray your Feelings: Reading Emotional States Through the Analysis of Spontaneous 3D Dynamics of Whole Body Motion (Y. Delevoye-Turrell and D. Lewkowicz)}

Body language is a particularly challenging area of research because of the complexity of biological motion. Indeed, the human body is a tool for intentional actions (e.g., walking to close the door) but also the medium for emotional expression (e.g., running under fear). Recent 3D kinematic studies have shown that social intention modulates the timing of 3D body kinematics. In the present study, while controlling for intention, we aimed to reveal the effects of emotional states on the timing properties of 3D body kinematics when walking at a preferred and natural pace. We will report results indicating how timing properties 
are affected by the 5 basic emotions. Furthermore, we will suggest that emotion valence impacts specifically movement fluency and cross-body coordination, factors that could be implemented in algorithms for the automatic detection of emotional states across a multitude of real-life situations.

\title{
3. Enhancing Spontaneous Synchronization of Cyclists' Pedal
}

Cadence to External Music, Through Sonification of Motor Rhythms (P.-J. Maes, V. Lorenzoni and J. Six)

It is well known that music stimulates human movement. Thereby, people tend to entrain their movements to musical patterns, such as the musical beat ('tactus'). This principle of 'spontaneous synchronization' has been applied in strategies to (unconsciously) adapt the tempo of rhythmical movement activities. However, this tendency for spontaneous synchronization does not seem to be consistent across people and types of rhythmical activity. In the present study, we test novel strategies to reinforce cyclists' spontaneous tendency to synchronize their pedal cadence (motor rhythm) to an external beat (musical rhythm), and consequently, to adapt their pedal cadence in predictive manners. These strategies rely on the sonification of cyclists' motor rhythms, allowing them to have an active contribution to the external music. Accordingly, we hypothesize that cyclists will be "attracted" to synchronized auditory-motor states as only these states lead to rewarding (synergistic) musical outcomes.

\section{Predicting Individual Response to Rhythmic Auditory Cueing in Parkinson's Disease (S. Dalla Bella, V. Cochen de Cock, D. Dotov, S. Bayard, C. Geny, P. Ihalainen and B. Bardy)}

Gait dysfunctions in Parkinson's disease (PD) can be partly relieved by rhythmic auditory cueing. This consists in asking patients to walk with a rhythmic auditory stimulus like a simple metronome or music. This beneficial effect on gait is visible immediately during the stimulation in terms of increased speed and stride length. In spite of the beneficial effects of cueing at the group level, however, individual patients tend to react differently to auditory cues. In this study we measured the response of 39 patients with PD and 39 controls to auditory rhythmic cues (metronomes and music), and their rhythm perception and synchronization abilities. Gait spatio-temporal parameters were recorded while the participants walked together with rhythmic cues. Only some patients clearly benefitted from cueing while others exhibited deleterious effects of the stimulation. This effect depends on patients' rhythmic abilities. These findings pave the way to devising individualized gait rehabilitation protocols.

\section{Timing and Time Perception in Children}

\author{
Sylvie Droit-Volet ${ }^{1}$, Florie Monier ${ }^{1}$, Jennifer T. Coull ${ }^{2}$ \\ and Laurence Casini ${ }^{3}$
}


1Université Clermont Auvergne, Laboratoire de Psychologie Sociale et Cognitive, CNRS, Clermont-Ferrand, France

${ }^{2}$ Laboratoire de Neurosciences Cognitives (LNC), Aix-Marseille Université, CNRS

(UMR7291), Marseille, France

${ }^{3}$ Aix-Marseille Université, CNRS, Marseille, France

\section{Symposium organizer: Sylvie Droit-Volet}

A symposium focused on timing and time perception in children.

\section{Keywords}

Babies, children, timing, cognition, disorder

\section{Different Developmental Trajectories for Different Time Judgments (S. Droit-Volet)}

There is not one but different types of judgment of time. Developmental studies in children aged from 5 to 9 years and adults have shown that developmental trajectories change between implicit and explicit timing. In implicit timing task, temporal variability remains constant across different ages but changes with age in explicit timing task, although the rhythm of development depends on the task used. Moreover, for explicit judgments of time, children are more prone to time distortion, being highly sensitive to contextual effects. These age-related changes in timing are significantly linked to the development of general cognitive capacities, in terms of working memory and attention. We will, therefore, discuss the development as an enrichment of separate forms of representation of time that are increasingly integrated though the development of awareness of the passage of time.

\section{Action Helps Young Children Maintain a Robust Representation of Time in Memory (F. Monier, S. Droit-Volet and J. T. Coull)}

Studies have shown that motor reproduction of a rhythm improves with age, and is linked to cognitive and motor development. However no study has yet examined whether developmental progress influences the motor precision of rhythm production, the temporal encoding of the inter-stimuli interval (ISI), or some combination of the two. 5 and 8 year old children watched a rhythmic sequence of dots (800 ms ISI). Within each age group, half of the participants tapped coincidently with the onset of the dots (visuo-motor learning), whereas the other half simply looked at these dots (visual learning). Upon extinction of the visual input, children reproduced this rhythm three times in a row, without re- presentation of the visual rhythm. Children's individual motor and memory capacities were assessed with neuropsychological tests. Our results showed that although 5 year olds were globally less accurate than 8 year olds, all children accurately reproduced the rhythm at least during the first reproduction trial. During the second and the third trials, on the other hand, tapping rate accelerated, especially in the younger children. Moreover, performance in the second and third trials was significantly less accurate for 5 year olds in the visual learning condition than for those in the visuo-motor one. Visuo-motor learning thus 
improved the ability to maintain the representation of the rhythm over the three trials. Correlation with neuropsychological measures confirmed this interpretation, revealing that temporal performance in the first reproduction trial was significantly linked to fine motor abilities, whereas temporal performance in the second and third trials was related to short-term and working memory capacity. Consequently, it seems that motor synchronization not only improves the motor component of rhythm reproduction, but also reinforces representation of the learned interval in memory.

\section{Time, Speech, and Dyslexia (L. Casini)}

The prevailing view concerning the cause of dyslexia in children points to phonological processing difficulties. However, it is still unclear whether the phonological deficit is the primary cause of dyslexia or whether it is secondary to impairments in the processing of more basic acoustic parameters of the speech signal. A recent theory of dyslexia, the temporal sampling theory, has put back temporal processing in the center of attention (Goswami, 2011). It assumes that deficits in temporal sampling could explain abnormal phonological development in children with dyslexia. In this talk, I will present some studies investigating the link between time, speech and dyslexia, and I will attempt to shed new light on temporal processing deficits in dyslexia by using the theoretical and methodological tools of time perception.

\section{Reference}

Goswami, U. (2011). A temporal sampling framework for developmental dyslexia. Trends Cogn. Sci., 15, 3-10. 


\title{
TALKS
}

(in order of presentation)

\section{Implicit Temporal Predictability Improves Auditory Pitch Discrimination Sensitivity}

\author{
Sophie K. Herbst ${ }^{1,2}$, Virginie van Wassenhove ${ }^{2,3,4}$ and Jonas Obleser ${ }^{1}$ \\ ${ }^{1}$ Department of Psychology, University of Luebeck, Germany \\ ${ }^{2}$ CEA, NeuroSpin, France \\ ${ }^{3}$ INSERM, U992, Cognitive Neuroimaging Unit, Université Paris-Sud, France \\ ${ }^{4}$ Université Paris-Saclay, F-Gif/Yvette, France
}

The human brain automatically extracts temporal contingencies from the environment, that is, rhythms or indicative durations, which allow the prediction of future events. While numerous studies have targeted rhythmic temporal contingencies, others have assessed temporal predictability by manipulating the time interval between cue and target in a trial-by- trial design, i.e., the foreperiod. Commonly, the use of temporal predictability in foreperiod paradigms has been promoted by using a few discrete durations, or by making participants aware of the temporal contingencies with explicit temporal cueing. Here, we aimed at testing the use of temporal predictability in a more naturalistic scenario, in which probabilistic variations of foreperiods were introduced implicitly, that is, without giving explicit temporal cues or informing participants of the relevance of timing. To this end, we varied foreperiods in an auditory pitch discrimination task in three independent EEG and psychophysical experiments. Unanimously, all three studies provide evidence that implicit probabilistic temporal predictability is used: Predictability sped up response times, or, depending on the experimental paradigm, improved pitch discrimination sensitivity. Concerning the neural signatures of temporal predictability, comparing predictive with non- predictive experimental conditions revealed a reduced cue-related evoked P2 response and an increased central alpha $(7-12 \mathrm{~Hz})$ power during predictive foreperiods. We also expected to find entrainment of the slow frequency at the stimulation rate, so that the target presented at the predicted time point would fall at the preferred phase of the entrained oscillation. However, this was not the case in the first two studies, suggesting that this mechanism might be contingent on more explicit forms of temporal predictability or more rhythmic scenarios. The third study was designed for a model based EEG-approach, to potentially reveal subtle effects of temporal predictability with the phase of neural oscillations, or, allowing us to underline the previous conclusions that these effects are contingent on more rhythmic paradigms.

\section{Keywords}

Prediction, behaviour, EEG, adult, 100s of ms-secs 


\title{
Saccadic Inhibition as an Index of Anticipation in a Discrimination Task
}

\author{
Roy Amit ${ }^{1}$, Dekel Abeles ${ }^{2}$, Marisa Carrasco ${ }^{3}$ \\ and Shlomit Yuval-Greenberg ${ }^{1,2}$ \\ ${ }^{1}$ Sagol School of Neuroscience, Tel Aviv University, Israel \\ ${ }^{2}$ School of Psychological Sciences, Tel Aviv University, Israel \\ ${ }^{3}$ Department of Psychology and Center for Neural Science, New York University, USA
}

Predicting the timing of upcoming events improves resource allocation and action preparation. Temporal anticipation is most commonly estimated using behavioral measures: when visual stimulus onset can be predicted, its perception is enhanced, as indicated by increased accuracy-rates and decreased reaction times in detection and discrimination tasks. However, these measures are indirect as they evaluate temporal anticipation retrospectively rather than index the process in real- time. A recent study (Dankner et al., in press) has shown that temporal predictions can be assessed directly by measuring prestimulus suppression of saccades in a detection task. Here we investigated whether the inhibition of fixational saccades can serve as an index of temporal anticipation in a discrimination task and compare to behavioral indices of temporal sensitivity. In each trial, observers were requested to discriminate the orientation of a central slightly tilted grating stimulus. This stimulus was preceded, with a varying stimulus-onset-asynchrony (SOA), by a cue, which was either informative or non-informative regarding the timing of the target. Our results showed that fixational saccades were suppressed before a predictable, but not before an unpredictable stimulus. This effect decreases linearly as SOA duration increases and is more robust than accuracy or RT measures. Additionally, a Bayesian classifier was trained to predict the stimulus' class (predictable/unpredictable) based on the single-trial oculomotor events preceding their onset with $63.8 \%$ mean accuracy across subjects. We conclude that the oculomotor system is sensitive to anticipation, and that saccadic inhibition can be used as an index for temporal anticipation, even when little or no effect is seen in other measures.

\section{Keywords}

Prediction, duration, behavior, electrophysiology, adult, 100 s of ms-secs

\section{Reference}

Dankner, Y., Shalev, L., Carrasco, M., \& Yuval-Greenberg, S. (in press). Pre-stimulus inhibition of saccades in adults with and without ADHD as an index for temporal expectations. Psychol. Sci. 


\title{
Neurological Evidence of a Dual Origin of the Foreperiod Effect
}

\author{
Bertrand Degos ${ }^{1,2}$, Ilhame Ameqrane ${ }^{3,4}$, Sophie Rivaud-Péchoux ${ }^{4}$, \\ Pierre Pouget ${ }^{4}$ and Marcus Missal ${ }^{3,4}$
}

${ }^{1}$ Neurology department, Parkinson's disease expert centre, Salpêtrière Hospital AP-HP, Paris, France

${ }^{2}$ Center for Interdisciplinary Research in Biology, Collège de France, INSERM U1050, CNRS UMR7241, Labex Memolife, Paris, France

${ }^{3}$ Institute of Neuroscience (IONS), Cognition and Systems (COSY), Université catholique de Louvain, Brussels, Belgium

${ }^{4}$ Sorbonne Universités, UPMC Univ Paris 06, Inserm U1127, CNRS UMR 7225, UM 75, ICM, F-75013 Paris, France

We investigated the foreperiod effect on the latency of saccadic eye movements in Parkinson's disease (PD) patients. Patients suffered either of the idiopathic (referred to as 'iPD') or of a rare genetic variant of the disease (referred to as 'Parkin'). In controls and in all patients saccadic latencies were longer for short foreperiods and decreased for longer ones, showing an oculomotor instantiation of the variable foreperiod effect. However, in controls and Parkin patients, the latency of saccades was also influenced by the duration of the previously experienced foreperiod. On average, this temporal short-term memory effect was not observed in iPD patients, either ON or OFF L-Dopa therapy. We suggest that the influence of the hazard rate on movement preparation is not affected in PD and is not dopaminergic-dependent. However, the absence of a contribution of short-term temporal memory to the FP effect in iPD patients clearly shows the dual origin of the foreperiod effect.

\section{Prospects of a Multiple Trace Theory of Temporal Preparation}

\author{
Sander Los, Wouter Kruijne and Martijn Meeter \\ Vrije Universiteit Amsterdam, The Netherlands
}

In warned reaction time tasks, the warning stimulus (S1) initiates a process of temporal preparation, which promotes a speeded response to the impending target stimulus (S2). Classic theories assume that temporal preparation is under strong voluntary control, informed by the distribution of S1-S2 intervals. However, our recently developed multiple trace theory of temporal preparation (MTP) offers a more mechanistic insight into 
preparation, based on simple associative learning rules. Here, we present recent experiments where different groups of participants experienced either the exponential or antiexponential distribution of S1-S2 intervals during an acquisition phase. This was followed by a test phase where all participants received, after explicit instruction, the uniform distribution. In this phase we found highly persistent transfer effects from the acquisition phase. These long-term learning effects are hard to reconcile with classic theories of temporal preparation and provide strong support for MTP.

\title{
Task-Oriented Optimal Inference in Interval Timing
}

\author{
Zhenguang Cai ${ }^{1}$ and Maarten Speekenbrink ${ }^{2}$ \\ ${ }^{1}$ School of Psychology, University of East Anglia, UK \\ ${ }^{2}$ Department of Experimental Psychology, University College London, UK
}

Resent research on interval timing has taken a Bayesian inference approach, according to which people make an optimal inference about the magnitude of a duration based on their noisy perception and their prior temporal experience (Cicchini et al., 2012; Jazayeri \& Shadlen, 2010). However, the specific mechanism behind Bayesian inference in timing is yet to be explored. We propose that people make task-oriented optimal inferences. In time reproduction, for instance, people infer how much time to be reproduced (rather than how much time there is in the stimulus) based on both the perceived stimulus duration and their prior reproduction experience (rather than their prior perception experience). This proposal thus contrasts with existing Bayesian timing accounts where the inference is an updated perceived duration in light of prior perception experience (Petzschner et al., 2015). To test the proposal, we conducted three set of experiments using time reproduction. In particular, we make use of the range bias (a duration is reproduced as longer if it follows longer than shorter prior durations), which has been shown to be driven by the use of prior experience in inferences (Jazayeri \& Shadlen, 2010). The first two experiments show that it is the reproduction rather than perception experience that constitutes the prior and drives the range bias in time reproduction. The second set of two experiments interleave motor vs. auditory reproduction in the same session and show that people resort to task-specific priors (based on prior motor or auditory reproductions) such that the different task-specific priors leads to a range bias. Finally, two experiments further show that reproduction is influenced by the reproduced but not perceived duration in the preceding trial, suggesting a trial-by-trial updating of the prior based on reproduction experience. All these results support the task- oriented optimal inference account.

\section{Keywords}

Duration, behavior, adult, 100s of ms-secs, Bayesian inference, range bias

\section{References}

Cicchini, G. M., Arrighi, R., Cecchetti, L., Giusti, M., \& Burr, D. C. (2012). Optimal encoding of interval timing in expert percussionists. J. Neurosci., 32, 1056-1060. 
Jazayeri, M., \& Shadlen, M. N. (2010). Temporal context calibrates interval timing. Nat. Neurosci., 13, 1020-1026.

Petzschner, F. H., Glasauer, S., \& Stephan, K. E. (2015). A Bayesian perspective on magnitude estimation. Trends Cogn. Sci., 19, 285-293.

\title{
Human Perceived Timing Follows Principles of Bayesian Inference
}

\author{
Darren Rhodes ${ }^{1,2}$ and Massimiliano Di Luca ${ }^{1}$ \\ ${ }^{1}$ Centre for Computational Neuroscience and Cognitive Robotics, School of Psychology, \\ University of Birmingham, Edgbaston, Birmingham, UK \\ ${ }^{2}$ Sackler Centre for Consciousness Science, School of Engineering \& Informatics, \\ University of Sussex, Brighton, UK
}

The timing of events is fundamental for everyday life, yet how humans perceive time remains unknown. Here we present a Bayesian inference model for the perceived timing of stimuli that combines noisy measurements with dynamic prior expectations. Quantitative validation of the model is difficult, as the precise noise characteristics of the priors and likelihoods are unknown, yet here we are able to infer such characteristics from psychophysical data. The model predicts that stimuli presented earlier or later than expected are reported closer to the expected timing, an effect that should increase with unreliable information. We presented sequences of regularly timed auditory stimuli with alternating intensities and asked participants to report whether the final stimulus appeared before or after a visual probe. The final stimulus was either of high or low intensity so to vary the reliability of estimating perceived timing. In line with the predictions of the model, we find that perceived simultaneity of stimuli is drawn towards the expected time point and the effect is larger for low-intensity stimuli. The novel account we propose is a viable alternative to existing models of perceived timing. The framework is general and can be applied to a wide spectrum of investigation in the psychology and neuroscience of time perception.

Keywords

Bayesian inference, timing, rhythm, uncertainty, perception, perceived timing

\section{How Pain Affects Time Estimation. A Physiological Study}

\author{
Andrea Piovesan, Laura Mirams, Helen Poole, David Moore, \\ Michael Richter and Ruth Ogden
}

Liverpool John Moores University, UK 
Time experience is known to be distorted by emotional stimuli (Droit-Volet et al., 2013). A proposed explanation for this is that emotional stimuli affect arousal. Indeed it is often suggested that arousal moderates time experience (Gil \& Droit-Volet, 2012). However, the relationship between physiological arousal and perceived duration is often assumed rather than tested. In a series of studies, the relationship between physiological arousal and temporal perception was directly tested. Healthy adults were asked to judge either 1) the duration of a painful electric shock, or, 2) the duration of neutral visual stimulus whilst in a prolonged state of pain. In both cases, participants experienced different subjective pain intensities (previously rated using a Numerical Rating Scale) to induce different arousal levels. Measures of skin conductance level and high-frequency heat-rate variability were also recorded as indicators of the sympathetic and parasympathetic arousal, respectively. Results indicate that sympathetic arousal is related to temporal judgments. However, this was true only when the arousing stimulus is the to-be-timed stimulus (electric shock). When arousal originates from a secondary, non-timed source, arousal may have the capacity to distract from the process of timing.

\title{
Keywords
}

Duration, behavior, physiology, adult, 100s of ms-secs

\section{References}

Droit-Volet, S., Fayolle, S., Lamotte, M., \& Gil, S. (2013). Time, emotion and the embodiment of timing. Timing Time Percept., 1, 99-126.

Gil, S., \& Droit-Volet, S. (2012). Emotional time distortions: The fundamental role of arousal. Cogn. Emot., 26, 847-862.

\section{Caloric Rewards Alter Time Perception and Time- Dependent Decision Making}

\author{
Bowen J. Fung, ${ }^{1,2}$, Carsten Murawski ${ }^{2}$ and Stefan Bode ${ }^{1}$ \\ ${ }^{1}$ Melbourne School of Psychological Sciences, The University of Melbourne, Victoria, \\ Australia \\ ${ }^{2}$ Department of Finance, The University of Melbourne, Victoria, Australia
}

Time is a critical component of many important decision-making processes. One example of this is in foraging behaviour, which requires a trade-off between the value of potential future rewards and the opportunity cost of time. However, not much is known about the subjective representation of time in these decision-making processes. Furthermore, the importance of accurate time perception in decision making seems at odds with our general experience of time, which is highly labile to both external and internal factors. Some researchers have previously suggested that distortions in our sense of time allow us to make better decisions by flexibly adapting to the current ecological context. For example, individuals have been shown to underestimate the duration of images of food, and 
this underestimation is larger for subjects who are more hungry (Gable \& Poole, 2012). One potential explanation is that time might be underestimated in order to minimise the perceived cost of acquiring food (if durations are under- estimated, the pursuit of reward might be prolonged). Here I report two studies that explored the psychophysiological relationships between biologically relevant rewards, time perception, and ecological, timedependent decision making. In the first study, we recruited 125 participants and assessed their performance on a novel variant of a duration production paradigm. While they performed this task, participants received different magnitudes of rewards on a trial-by-trial basis. We tested four different types of liquid primary rewards (fruit juice, water, aspartame, and maltodextrin) and a secondary reward (money). We found that consumption of the primary rewards containing calories lead to systematic overproductions of intervals (from 2-5 seconds). This pattern of results suggested that biologically relevant rewards were able to alter time perception (Fung et al., 2017b). In the second study, we recruited 50 participants to investigate whether caloric primary rewards were also able to bias timedependent decision making. Participants fasted for four hours, and then completed a task similar to a patch-leaving foraging paradigm, incentivised with monetary rewards. Participants who consumed a caloric drink in between blocks gave up waiting for rewards significantly earlier, compared to those who consumed water (i.e., participants who consumed the caloric drink were less patient.) These results suggested that the consumption of biologically relevant rewards was able to alter time-dependent decision making, despite the fact that the drink was irrelevant to the task (Fung et al., 2017a). These results support the idea that time perception can be affected by an individual's homeostatic state, and further suggest that different homeostatic states can influence time-dependent decision making processes. Taken together, these experiments imply that our experience of time may be part of a psychophysiological mechanism whereby energy levels affect perceived time, which, in turn, may act to optimise ecological decision making.

\title{
Keywords
}

Reward, decision-making, duration, behavior, adult, secs-mins

\section{References}

Gable, P. A., \& Poole, B. D. (2012). Time flies when you're having approach-motivated fun: Effects of motivational intensity on time perception. Psychol. Sci., 23, 879-886.

Fung, B. J., Bode, S., \& Murawski, C. (2017a). High monetary reward rates and caloric rewards decrease temporal persistence. Proc. R. Soc. Lond. B Biol. Sci., 284.

Fung, B. J., Murawski, C., \& Bode, S. (2017b). Caloric primary rewards systematically alter time perception.J. Exp. Psychol. Hum. Percept. Perform.

\section{Temporal Representations in the Duration Discrimination Task}

\author{
Başak Akdoğan ${ }^{1}$, Randy Gallistel ${ }^{2}$, Ben Gersten ${ }^{1}$, Amita Wanar $^{3}$ \\ and Peter Balsam ${ }^{3,4}$
}




\author{
${ }^{1}$ Department of Psychology, Columbia University, USA \\ ${ }^{2}$ Department of Psychology, Rutgers University, USA \\ ${ }^{3}$ Department of Psychology, Barnard College, Columbia University, USA \\ ${ }^{4}$ New York State Psychiatric Institute, USA
}

\begin{abstract}
Temporal information-processing is critical for adaptive behavior and goal-directed action. It is thus crucial to understand how the temporal distance between behaviorally relevant events is encoded. To this end, we tested mice in a duration discrimination procedure in which they learned to correctly categorize tones of different durations as short or long. To investigate the nature of temporal representations, after being trained on a pair of target intervals they transferred to conditions in which the absolute and/or relative relations of target intervals and corresponding response locations were systematically manipulated. The adjustments of choices to these changes in temporal and spatial contingencies were characterized with respect to acquisition, learning rates, temporal sensitivity, and response times associated with short and long categorizations. These indices of timing behavior collectively revealed that although subjects appear to have knowledge of absolute durations, the transfer occurred most readily when the relative mapping between durations and corresponding response locations was maintained. The findings indicate that the default representations in a duration discrimination task encode relative rather than the absolute information about time.
\end{abstract}

\title{
Keywords
}

Duration, behavior, modeling, animal (mice), secs-mins

\section{The Relation between Symbolic and Non-Symbolic Representations of Time}

\section{Karina Hamamouche and Sara Cordes}

Boston College, USA

Studies have identified a relation between non-symbolic numerical abilities and formal mathematics (see Halberda et al., 2008), yet no work has examined whether a similar relation between informal, non-symbolic and formal, symbolic abilities exists in other quantities domains, such as time. That is, does acquiring a symbolic understanding of time (i.e., identifying time on a clock or accurately estimating how long it takes to complete everyday activities) relate to improvements on basic timing tasks? To test this hypothesis, 6-7 year old children $(\mathrm{N}=40$; Mage $=7.07$ years, 22 males $)$, who are in the process oflearning symbolic time concepts in school, completed a temporal discrimination task, a temporal estimation task, and a symbolic timing assessment (which consisted of reading analog and digital clocks, estimating how many minutes it takes to complete everyday activities, etc.). Parents also completed a survey regarding their child's symbolic timing experiences and activities. Results revealed that, when controlling for age, children's symbolic timing 
performance was independently correlated with both non-symbolic discrimination performance $(\mathrm{r}=.508, \mathrm{p}=.002)$ and estimation performance $(\mathrm{r}=-.421, \mathrm{p}=.013)$. Further, age $(\beta=.648, p<.001)$, discrimination performance $(\beta=.313, p=.004)$, and estimation performance $(\beta=-.241, \mathrm{p}=.020)$ each uniquely predicted performance on the symbolic timing task when controlling for the other variables. Parent surveys, however, were unrelated to any of the child measures (p's $>.18$ ). Paralleling findings in the numerical domain, this work is the first to identify a relation between symbolic and non-symbolic temporal abilities. Future work using experimental designs is necessary to determine whether this relation is causal.

\title{
Keywords
}

Duration, behavior, children, 100s ms-secs, secs-mins

\section{Reference}

Halberda, J., Mazzocco, M. M., \& Feigenson, L. (2008). Individual differences in non- verbal number acuity correlate with maths achievement. Nature, 455, 665-668.

\section{Modality Specific Rate Aftereffects: Evidence towards Distributed Timing Mechanisms}

\author{
Aysha Motala and David Whitaker \\ School of Optometry and Vision Sciences, Cardiff University, Cardiff, UK
}

Time perception is important for a number of different tasks, some of which include understanding speech and appreciating music. However, it remains to be elucidated whether sensory time perception occurs due to a central timing component overlooking each sense or rather, that distributed mechanisms exist each specifically dedicated to each sense, and operating in a largely independent manner. To this end, a range of unimodal and crossmodal rate adaptation experiments were conducted, in which adapting to a fast rate makes a moderate rate feel slow, and adapting to a slow rate, makes the same moderate rate feel fast. Rate perception was quantified by a method of temporal reproduction across the visual, auditory and tactile senses on three observers. Repulsive rate aftereffects were observed across all unimodal conditions however, no such effects were observed for any cross-modal pairings. A similar pattern of results was found in subsequent experiments when subjects were unaware of which sensory modality would form the test stimulus. We use the current findings to suggest that sensory timing abilities are open to change, but crucially, that this change is modality-specific and finally, that our data supports the distributed theory of timing..

\section{Keywords}

Rhythms, multisensory, behavior, 10s-100s of ms, 100s of ms-secs 


\title{
Observers Adapt to the Physical, not the Perceived Duration of Visual Events
}

\author{
Jim Maarseveen', Chris L.E. Paffen', Frans A. J. Verstraten ${ }^{1,2}$ \\ and Hinze Hogendoorn ${ }^{1}$
}

\author{
${ }^{1}$ Department of Experimental Psychology, Helmholtz Institute, Utrecht University, \\ Utrecht, The Netherlands \\ ${ }^{2}$ School of Psychology, Faculty of Science, University of Sydney, Sydney, Australia
}

Recent evidence suggests that duration selective channels underlie the encoding of duration (Heron et al., 2012). Here, we investigated whether these duration selective channels encode the time between the onset and offset of a stimulus, or the perceived duration of a stimulus. To this end, we adapted participants to stimuli that differ in their physical and perceived duration. More specifically, we used the Temporal Frequency-Induced Time Dilation illusion in which the perceived duration of stimuli increases with increasing temporal frequency content, without changing the perceived onset and offset of those stimuli (Kanai et al., 2006; Kaneko \& Murakami, 2009). Participants adapted to repetitions of a rotating radial grating (illusion-inducing stimulus); a static radial grating matched to the physical duration of the illusion- inducing stimulus (physically matched stimulus); or a static radial grating matched to the perceived duration of the illusion-inducing stimulus (perceptually matched stimulus). We then measured the resulting duration after-effect using a cross-modal duration judgment task in which participants compared the duration of an auditory reference to that of a static visual test stimulus. We found that participants adapted to the physical, and not the perceived duration of our illusion-inducing stimulus: the duration after-effect for the illusion-inducing stimulus did not differ from the after- effect for the physically matched stimulus; but did differ from the after-effect for the perceptually matched stimulus. This result demonstrates that duration encoding is based on the time difference between stimulus onset and offset and does not necessarily reflect the perceived duration of a stimulus.

\section{Keywords}

Duration, behavior, adult, 10 s to 100 s of $\mathrm{ms}$

\section{References}

Heron, J., Aaen-Stockdale, C., Hotchkiss, J., Roach, N. W., McGraw, P. V., \& Whitaker, D. (2012). Duration channels mediate human time perception. Proceedings. Biological Sciences / the Royal Society, 279, 690-698.

Kanai, R., Paffen, C. L., Hogendoorn, H., \& Verstraten, F. A. (2006). Time dilation in dynamic visual display.J. Vis., 6, 1421-1430.

Kaneko, S., \& Murakami, I. (2009). Perceived duration of visual motion increases with speed.J. Vis., 9, 14. 


\title{
Rapid Recalibration to Audiovisual Asynchronies Occurs Unconsciously
}

\author{
Erik Van der Burg ${ }^{1,2}$, David Alais ${ }^{2}$ and John Cass $^{3}$ \\ ${ }^{1}$ Dept. of experimental and applied psychology, Vrije Universiteit Amsterdam, \\ The Netherlands \\ ${ }^{2}$ School of psychology, University of Sydney, Australia \\ ${ }^{3}$ School of psychology, Western Sydney University, Australia
}

In natural scenes, audiovisual events deriving from the same source are synchronized at origin. However, from the perspective of the observer, there are likely to be significant multisensory delays due to physical and neurological differences. Fortunately, our brain appears to compensate for the resulting latency differences by rapidly adapting to the asynchronous audiovisual events (Van der Burg, Alais \& Cass, 2013; Van der Burg \& Goodbourn, 2015). Here, we examine whether rapid recalibration to asynchronous signals occurs unconsciously. On every trial, a brief tone pip and flash were presented across a range of stimulus onset asynchronies (SOAs). Participants were required to perform two tasks in alternating order. On adapter trials, participants judged the order of the audiovisual events. Here, audition either lead or lagged vision with a fixed SOA (150 ms). On test trials the SOA as well as the modality order varied randomly, and participants judged whether the events were synchronized or not. For test trials, we show that the point of subjective simultaneity (PSS) follows the physical rather than the perceived (reported) modality order of the preceding trial. These results suggest that rapid temporal recalibration occurs unconsciously.

\section{Keywords}

Multisensory processing, synchrony judgment, recalibration, audiovisual

\section{References}

Van der Burg, E., Alais, D., \& Cass, J. (2013). Rapid recalibration to asynchronous audiovisual stimuli. J. Neurosci., 33, 14633-14637.

Van der Burg, E., \& Goodbourn, P. T. (2015). Rapid, generalized adaptation to asynchronous audiovisual speech. Proc. R. Soc. Lond., B, 282, e20143083.

\section{Time Order is a Psychological Bias}

\section{Laetitia Grabot ${ }^{1}$, Anne Kösem ${ }^{2,3}$, Leila Azizi ${ }^{1}$ and Virginie van Wassenhove ${ }^{1}$}

${ }^{1}$ Cognitive Neuroimaging Unit, Institut National de la Santé et de la Recherche Médicale, Université Paris-Saclay, NeuroSpin, Commissariat à l'Energie Atomique et aux Energies Alternatives, Université Paris-Saclay, France 

${ }^{2}$ Radboud University, Donders Institute for Brain, Cognition and Behaviour, Nijmegen, The Netherlands
${ }^{3}$ Neurobiology of language, Max Planck Institute for Psycholinguistics, Nijmegen, The Netherlands

Inter-individual variability in temporal order perception has rarely been tackled empirically although readily observed in the literature. Classically, temporal order perception has been studied using temporal order judgments (TOJ) in which participants report the order of two stimuli presented few milliseconds apart. The temporal delay, at which participants consider the order of stimuli to be at chance is called Point of Subjective Simultaneity (PSS) and is typically averaged across participants canceling out potential individual effects. Here, we asked whether an individual's PSS was stable over weeks, which would indicate an intrinsic or hardwire constant delay in perceptual systems. The present work aimed to test this stability and to distinguish this hypothesized intrinsic constant from an attentional effect, considering that temporal order perception is known to be modulated by attentional fluctuations. We designed a longitudinal psychophysics study using auditory, visual, and audiovisual TOJs. The experimental design also comprised unisensory (vision or audition attended) and divided (audition and vision attended) attentional conditions. A standard measure of PSS was extracted from the divided attentional condition, and a measure of PSS free of any attentional biases was computed from the unisensory attentional conditions. Our results show that individual standard PSS are stable over months, strengthening the hypothesis that the PSS is an individual marker of temporal perception and thus a defining feature of an individual's core biases. Attention could partially, but not fully, compensate for this bias. We measured the neural oscillatory activity with magnetoencephalography during a TOJ task and this study revealed that perception of temporal order cannot be predicted if the individual biases are not taken into account. These results shed a new light in the debated issue of what is actually measured in a TOJ and stressed the importance to tackle individual differences.

\title{
Keywords
}

Order, multisensory, behavior, MEG, adult, 10s-100s of ms

\section{Reframing Variability in Auditory, Visual, and Audiovisual Timing Tasks: From Nuisance to an Aid to Understand Complex Systems Dynamics}

\author{
Lars T. Boenke ${ }^{1,2}$, Richard Höchenberger ${ }^{3}$, David Alais ${ }^{2}$ \\ and Frank W. Ohl l,4,5 \\ ${ }^{1}$ Leibniz Institute for Neurobiology, Magdeburg, Germany \\ ${ }^{2}$ University of Sydney, Sydney, Australia \\ ${ }^{3}$ German Institute of Human Nutrition Potsdam-Rehbrücke, Germany
}




\author{
${ }^{4}$ Otto-von-Guericke Universität Magdeburg, Magdeburg, Germany \\ ${ }^{5}$ Center for Behavioral Brain Sciences (CBBC), Magdeburg, Germany
}

In modern behavioral science, audiovisual timing is regarded as the oldest example of unexplained variability across studies and across individuals. While conclusions are in general average-based and partly contradictive, variability is condoned as nuisance. While both, synchrony- (SJs) and temporal-order-judgments (TOJs), have been employed in tasks measuring the point of subjective simultaneity (PSS), the latter is found to show higher inter-individual variability than the former. This difference lacks explanation. A fundamental difference between both tasks is, that while SJs can be achieved by focusing only on the temporal relationship of stimuli, TOJs require focusing on an additional stimulus dimension (e.g., location, etc.). Processing additional stimulus dimensions need in general additional neural networks. We hypothesized that the combination of a larger number of different networks during a decision under uncertainty yield a larger number of possible states being randomly realized in each trial. Larger number of possible realizations, ceteris paribus, are accompanied by higher variability. To test for this hypothesis, we first performed a meta-study on SJ- and TOJ-tasks that have employed simple audiovisual stimuli, and plotted the average reported point PSS as a function of the number of participants employed in each study (considering each participant as one possible realization). Subsequently, we performed a within-participant study including auditory, visual, and audiovisual SJs and spatialized TOJs. Besides different task instructions, both tasks were otherwise identical. The meta-study revealed average PSS-values with a slight preference for the auditory modality (statistically indistinguishable between tasks). Moreover, the scatter of study-wise obtained PSS-values was funnel-shaped with a broader funnel for TOJs than for SJs (in line with bootstrap results in our study). Finally, simulations supported our overall results to be compatible with the notion that differing number of possible states yields differing variability irrespective of the level of observation (i.e., single-trials, between individuals or studies).

\title{
Saccadic Temporal Recalibration Leads to a Reversal of Cause and Effect
}

\section{Brent Parsons, Dunia Giomo and Domenica Bueti}

Scuola Internazionale Superiore di Studi Avanzati, Italy

Studies of saccadic adaptation have predominately focused on manipulations in the spatial dimension. Shifting the location of the saccade target midflight leads to changes in the motor command (e.g., saccade amplitude) and affects subsequent perceptual judgments (e.g., localization). Significant gain reduction has been reported even when the shifted target is presented at post-saccade delays of up to $400 \mathrm{~ms}$ (Shafer, Noto \& Fuchs 2000). Recent experiments manipulating only the temporal dimension, the delay between saccade landing and target presentation, have shown changes in peak velocity of the saccade 
(Shadmehr et al., 2010) and the duration of the post-saccadic stimulus (Parsons \& Ivry, 2014). The current study investigates whether adapting to these artificially induced delays leads to temporal recalibration between action and effect. Subjects made rapid alternating saccades with fixed delays between the end of the eye movement and the onset of the visual stimulus. After every fifth saccade, a probe stimulus was presented at one of several offsets around saccade ending. Subjects were asked if the probe onset occurred before or after their eye landed. Following adaptation to delays, subjects reported visual stimuli presented after the saccade ending as occurring before their saccade, evidence for saccadic temporal recalibration. The results provide novel insight into the mechanisms underlying perceptual stability and links saccades to the more general phenomenon of motor-sensory recalibration.

\title{
References
}

Shafer, J. L., Noto, C. T., \& Fuchs, A. F. (2000). Temporal characteristics of error signals driving saccadic gain adaptation in the macaque monkey.J. Neurophysiol., 84, 88-95.

Shadmehr, R., de Xivry, J. J. O., Xu-Wilson, M., \& Shih, T. Y. (2010). Temporal discounting of reward and the cost of time in motor control.J. Neurophysiol., 30, 10507-10516.

Parsons, B.D., \& Ivry, R.B. (2014). Perceptual consequences of delaying the post-saccadic target.J. Vis., 14, 1228-1228.

\section{Perceived Timing of a Visual Event Is Affected by Temporal Context}

\author{
Ljubica Jovanovic and Pascal Mamassian \\ Laboratoire des systèmes perceptifs, Département d'études cognitives, École normale \\ supérieure, PSL Research University, CNRS, Paris, France
}

Perceived timing of an event is affected by the context, as demonstrated with kappa effect (Cohen et al., 1953) or temporal ventriloquism (Fendrich \& Corballis, 2001). However, it is not clear how these phenomena can be explained by models of time perception. We present here a novel paradigm that enables us to precisely study how timing of a visual event is affected by other events in temporal proximity. Participants were initially familiarized with a fixed interval duration by watching the hand of a clock rotating at a constant speed, one cycle in 2 seconds. In the rest of the experiment, the hand was no longer presented and the clock face was represented as a circle. A stimulus was then briefly flashed within the interval duration, and participants had to place a cursor on the circle at the location where the hand would have been at the time of the flash. Stimuli were discs of different colors presented at the center of the clock. In most trials (91\%), two stimuli were presented and a stimulus color was shown at the end of the trial as a cue for reproduction. We varied the relative timing of target and distractors in ten steps (-300 to $300 \mathrm{~ms})$. In the remaining trials, only one stimulus was shown. In additional experiments, we assessed 
timing of a single event and sensory - motor noise in a pointing task. Estimated timing of a target was affected by timing of distractors: targets preceded by a distractor appeared earlier than those followed by it. Performance in the single event experiment was more accurate and less variable compared to the main experiment. These results are in the line with previous findings suggesting that timing of different events are interdependent (Fendrich \& Corballis, 2001) and affected both by temporal proximity as well as number of events.

\title{
References
}

Cohen, J., Hanse, C. E. M., \& Sylvester, J. D. (1953). A new phenomenon in time judgment. Nature, 172, 901.

Fendrich, R., \& Corballis, P. M. (2001). The temporal cross-capture of audition and vision. Percept. Psychophys., 63, 719-725.

\section{Perceived Timing of Sensory Events Triggering Actions in Parkinson's Disease}

\author{
Yoshiko Yabe $^{1,2}$, Penny A. MacDonald ${ }^{1}$ and Melvyn A. Goodale ${ }^{1}$ \\ 1The Brain and Mind Institute, The University of Western Ontario, Canada \\ ${ }^{2}$ Research Institute, Kochi University of Technology, Japan
}

Recently, we showed that the perceived timing of a sensory event that triggers an action was delayed, as if it were attracted towards the action. The fact that this delay in the perceived timing occurred even when an individual cancelled an action suggests that it is the motor programming rather than the action itself that affects the perceived time (Yabe \& Goodale, 2015). One can ask therefore: does this distortion in the perception of time of onset of a stimulus event disappear in Parkinson's disease (PD) patients as a result of their impairment in preparation of actions? In this study, we examined the subjective onset of sensory events that triggered actions in PD patients. Fourteen PD patients, 15 age-matched controls and fifteen young controls were tested on a Go/No-go task, both ON and OFF dopaminergic therapy on separate days for all groups. Participants were required to fixate a clock face with a rotating second hand at the center of a computer screen. In the action condition, participants were required to blow into a microphone when they heard an auditory tone in which the pitch indicated a Go trial. They were required to cancel their actions when they heard an auditory tone in which the pitch indicated a No-go trial. In the control condition, regardless of the pitch of the tone, they were asked not to blow into the microphone. The lag of perceived onset of the tone compared to the real timing was calculated for each trial. As a result, the difference in the lags between the action and control conditions were smaller in PD patients than in both control groups. Interestingly, in PD patients in OFF medication state, the difference in the lags was significantly smaller than zero. In short, time flew backward when PD patients intended to act. 


\title{
Reference
}

Yabe, Y., \& Goodale, M. A. (2015). Time flies when we intend to act: Temporal distortion in a go/no-go task. J. Neurosci., 35, 5023-5029.

\section{Actively Anticipating Upcoming Tempo Changes Modulates Induced Neural Beta Power}

\author{
Emily Graber ${ }^{1}$ and Takako Fujioka ${ }^{1,2}$ \\ ${ }^{1}$ Center for Computer Research in Music and Acoustics, Stanford University, Stanford, \\ California, USA \\ ${ }^{2}$ Neurosciences Institute, Stanford University, Stanford, California, USA
}

Investigation While playing and listening to music, musicians and listeners can not only track the beat of the music, but also anticipate upcoming tempo changes such as gradual increases or decreases in the beat rate (called 'accelerandi' or 'ritardandi'). In the present study, we investigated how active temporal anticipation in listening to auditory beat stimuli is related to neural oscillations in the beta range $(13-30 \mathrm{~Hz})$. Typically, beta-band dynamics are characterized by decreases in power prior to and during movement (eventrelated desynchronization, ERD), and increases in power after movement (event-related synchronization, ERS). However, induced beta-band power modulations are also related to anticipatory temporal processing; ERS rates scale in proportion to predictable isochronous tempi, peak amplitudes are altered by temporal expectation and attention, and ERD is modulated by imagined metrical structure. We hypothesized that while listening to isochronous beat sequences, active temporal anticipation for upcoming 'accelerandi', 'ritardandi' or 'no changes' could alter beta power modulation patterns according to the anticipated beat timing. EEG was recorded from musicians while they listened to beat sequences at $100 \mathrm{bpm}$ and anticipated gradual tempo changes according to informative cues presented before every sequence. The onset of the tempo change within a sequence was varied from trial to trial. In the 'no change' condition, beta power showed prototypical modulation patterns in line with previous findings (Fujioka et al., 2012). In contrast, the induced beta oscillations during directed anticipation were different prior to accelerations and decelerations in both ERD and ERS amplitudes in auditory and motor areas. In a separate experimental block, subjects identified cued tempo changes faster than uncued changes, confirming the facilitative effects of active anticipation. These results support the idea that beta synchronization and desynchronization play roles in top-down processes and that beta-band dynamics reflect active, predictive timing mechanisms.

\section{Keywords}

Prediction, music, EEG, adult, 10s-100s of ms, beta band

\section{Reference}

Fujioka, T., Trainor, L. J., Large, E. W., \& Ross, B. (2012). Internalized timing of isochronous sounds is represented in neuromagnetic beta oscillations. J. Neurosci., 32, 1791-1802. 


\title{
Neural Entrainment Reflects Temporal Predictions Guiding Speech Comprehension
}

\author{
Anne Kösem ${ }^{1,2}$ \\ ${ }^{1}$ Max Planck Institute for Psycholinguistics, Nijmegen, The Netherlands \\ ${ }^{2}$ Radboud University, Donders Institute for Brain, Cognition, and Behaviour, Nijmegen, \\ The Netherlands
}

During listening, low-frequency neural oscillations entrain to the dynamics of the speech signal, and neural entrainment has been hypothesized to be functionally relevant for speech processing. In this MEG experiment, the entrainment of oscillations in auditory cortex was manipulated to test its influence on speech perception. The manipulation was operated based on the hypothesis that neural entrainment reflects temporal predictions: the brain would internalize the rhythms of preceding signals to process the ongoing sensory input. Hence, ongoing neural oscillatory activity could be manipulated by changing the dynamics of past sensory stimulation. Using speech sentences that suddenly increased or decreased in rate, neural entrainment to past speech was expected to last after the speech rate changes, and to influence speech perception. The beginning of the sentence was either presented at a fast or a slow speech rate, while the last three words (target window) were displayed at an intermediate rate across trials. Dutch participants were asked to report the perception of the last word of the sentence, which was ambiguous with regards to its vowel duration (short vowel /a/ - long vowel /a:/ contrast). Sustained neural entrainment was reported after rhythmic stimulation: brain oscillatory activity that corresponded in frequency to the preceding speech rate was observed during the target window. The sustained neural entrainment correlated with speech perceptual biases: participants who showed stronger persisting neural entrainment were more influenced by the past speech rate in their perception of the last word. These findings provide empirical support for oscillatory models of speech processing, suggesting that neural oscillations actively modulate speech comprehension.

\section{Keywords}

Language, rhythms, MEG, adult, 100s of ms-secs

\section{Visual to Auditory Entrainment Enhances Auditory Gap Detection Performance}

\author{
Anna-Katharina R. Bauer ${ }^{1}$, Martin G. Bleichner, ${ }^{1,2}$, Sylvain Baillet ${ }^{3}$ \\ and Stefan Debener1,2
}

${ }^{1}$ Neuropsychology Lab, Department of Psychology, University of Oldenburg, Germany

${ }^{2}$ Cluster of Excellence Hearing4All, University of Oldenburg, Germany 
${ }^{3}$ McConnell Brain Imaging Centre, Montreal Neurological Institute, McGill University, Canada

Sounds are inherently characterized by their temporally evolving and rhythmic nature. However, our sensory systems are continuously receiving many inputs that are often interrelated. Indeed, visible events often cause subsequent sounds. The synchronization of neural oscillations in the auditory cortex induced by visual rhythmic stimulation is called cross-modal phase entrainment. In two experiments using electro-encephalography (EEG) and magnetoencephalography (MEG), we investigate how we integrate cross-modal rhythmic information over time and the influence of cross-modal phase entrainment on auditory gap detection performance. Listeners were presented with auditory-only $(\sim 4.33$ s) and visual-auditory stimuli ( $7 \mathrm{~s})$. Participants had to detect short silent gaps that were systematically distributed with respect to the phase of a $3 \mathrm{~Hz}$ frequency-modulated tone. In the visual-auditory condition, the visual stimulation consisted of a Gaussian pulsating circle, which preceded the auditory tone by $\sim 2 \mathrm{~s}$ and was presented either in or out of phase. We found that gap detection performance increased for the visual-auditory conditions relative to the auditory-only condition and accuracies were modulated by stimulus phase. Analysis of the power spectral density revealed spectral peaks at $3 \mathrm{~Hz}$ and the $6 \mathrm{~Hz}$ harmonic at the sensor level (EEG) and in visual and auditory regions of interest (MEG). ERP analysis on the sensor level and ERF analysis in the auditory ROI revealed higher amplitudes for detected gaps. Spectral analysis revealed enhanced power in the auditory cortex during visual stimulation (MEG). We showed that cross- modal phase entrainment leads to enhanced gap detection performance and neural entrainment effects. We will further present connectivity analysis between the visual and auditory cortices to identify the mechanism underlying cross-modal phase entrainment.

\title{
Keywords
}

Multisensory, rhythms, adult, secs-mins, MEG, EEG

\section{Parallel Preparation of Serial Timing for Skilled Sequence Production (MEG)}

\author{
K. Kornysheva', ${ }^{1,}$, D. Bush ${ }^{2}$, A. Meyer ${ }^{2}$, A. Sadnicka ${ }^{3}$, N. Burgess ${ }^{2}$ \\ and G. Barnes ${ }^{4}$ \\ ${ }^{1}$ School of Psychology, Bangor University, Wales, UK \\ ${ }^{2}$ Institute of Cognitive Neuroscience, University College London, London, UK \\ ${ }^{3}$ Institute of Neurology, University College London, London, UK \\ ${ }^{4}$ Functional Imaging Laboratory, University College London, London, UK
}

Monkey electrophysiology suggests that serial actions in skilled movements are prepared in parallel with the probability of corresponding neural pattern activations being weighted by their serial occurrence (Averbeck et al., 2002). This offers clear support for 
the competitive queuing account of motor sequence control. The current study aimed at testing 1) whether this effect could be reproduced in humans using non-invasive imaging (MEG) pattern classification and in how far the competitive queuing reflected the temporal structure of the planned sequences. Sixteen healthy participants were trained for two days to associate abstract visual cues with a subsequent production of the respective spatio-temporal sequence from memory following a go cue. Sequences followed a two-bytwo factorial design involving a combination of two spatial and two temporal features of sequences. As in previous serial reaction time paradigms (Kornysheva et al., 2013; Kornysheva \& Diedrichsen, 2014), training to produce spatio-temporal sequences from memory lead to the behavioural transfer of both spatial and temporal sequence features to new sequence combinations. The dynamic probability profiles for action specific MEG pattern activations confirmed previous electrophysiology results in monkeys suggesting parallel competitive queuing of sequential actions in the preparation period. Critically, the neuronal ordering reflected the temporal structure of sequences, independently of the specific actions employed in the sequence. Finally, the absolute probability distance between patterns related to neighboring actions during preparation predicted subsequent behavioural performance across subjects. In the high performing group the distance between adjacent action pattern probabilities during preparation was significantly higher than in the low performing group. Our results provide a fundamental insight into the neural preparation of temporally structured sequences in humans, and advocate a new neural signature of accurate preparation for skilled sequence performance.

\title{
References
}

Averbeck, B.B., Chafee, M.V., Crowe, D.A., \& Georgopoulos, A.P. (2002). Parallel processing of serial movements in prefrontal cortex. PNAS, 99, 13172-13177.

Kornysheva, K., \& Diedrichsen, J. (2014). Human premotor areas parse sequences into their spatial and temporal features. eLife, 3, e03043.

Kornysheva, K., Sierk, A., \& Diedrichsen, J. (2013) Interaction of temporal and ordinal representations in movement sequences. J. Neurophysiol., 109, 1416-1424.

\section{Neuronal Synchrony Mediates the Attention to Time and its Estimation}

\author{
Shrikanth Kulashekhar ${ }^{1,2}$, Matias J. Palva ${ }^{2}$ and Satu Palva ${ }^{2}$ \\ ${ }^{1}$ SISSA, Trieste, Italy \\ ${ }^{2}$ Neuroscience Center, University of Helsinki, Finland
}

The estimation of durations is crucial for perception, action, and cognition. Studies on timing in humans using functional magnetic resonance imaging (fMRI) have revealed a positive correlation between the estimation of multi-second temporal durations and neuronal activity in the sensory and motor areas, prefrontal and parietal cortices, basal ganglia and cerebellum (Muller \& Nobre, 2014). While the brain regions involved in the estimation of 
durations are fairly well known, the systems-level mechanisms coordinating the distributed neuronal activity in the timing areas have remained elusive. It has been suggested that synchronized oscillations could regulate communication in neuronal networks and hence serve such coordination. However, the role of synchronized oscillations in the estimation of multi-second time intervals has remained largely unknown (Muller \& Nobre, 2014). We recorded human neuronal activity using magnetoencephalography (MEG) during a temporal discrimination task adapted from Coull et al. (2004). A cue instructed the subjects to either estimate and compare the durations or in the contrast condition, the colours of dynamic visual stimuli. To address the functional significance of neuronal synchronization we identified frequency specific, large-scale synchronized networks from source reconstructed MEG data. Time estimation was associated with sustained long-range synchronization in the alpha- $(10-12 \mathrm{~Hz})$ and beta- $(14-30 \mathrm{~Hz})$ bands. We found the synchronization in-between the sensory and motor areas, prefrontal, parietal and visual cortices regions. In addition, we found that attending to the temporal aspect of the stimuli was associated with synchronization in the gamma band $(40-80 \mathrm{~Hz})$ between the sensory and motor areas. The results thus suggest that alpha and beta-band phase synchronization is related to the estimation of durations in the seconds' range, while gamma-band synchronization could regulate the coordination and maintenance of temporal attention.

\title{
Keywords
}

Duration, attention, rhythms, synchrony, MEG, adult, 100s of ms-secs

\section{References}

Coull, J. T., Vidal, F., Nazarian, B., \& Macar, F. (2004). Functional anatomy of the attentional modulation of time estimation. Science, 303, 1506-1508.

Muller, T., \& Nobre, A. C. (2014). Perceiving the passage of time: Neural possibilities. Ann. N. Y. Acad. Sci., 1326, 60-71.

\section{Anticipation of a Familiar Event Is Associated with Widespread Predictive Activation of Sensory Cortical Areas}

\author{
Dan Lloyd \\ Department of Philosophy and Program in Neuroscience, Trinity College, \\ Connecticut, USA
}

Anticipation and expectation are familiar aspects of subjective experience, but their ubiquity in experience makes them elusive to experimental manipulation. Functional MRI datasets in the Human Connectome Project (http://www.humanconnectome project.org/), however, offer a way in: Seventy adult subjects were scanned at 7 Tesla while watching 60 minutes of various movie clips. One of the segments, an 88 second montage, 
repeats four times during the scanning session. With the fourth repetition, the montage is very familiar and depicted events can be anticipated. Anticipation then can be isolated via the following analysis, based on a comparison of the first, unanticipated, presentation of the montage, with the fourth, highly anticipated, presentation. First, we identify voxel time series that are strongly correlated between the two presentations, that is, we identify the voxels that are very strongly associated with the depicted events in both the unfamiliar (first) and familiar (fourth) presentations of the same montage. Then we search for the presence of anticipatory activation in these voxels, as follows: For each, we offset the fourth segment time series in comparison to the montage, sliding it forward in time. In other words, we ask whether the voxel-wise response to the fourth segment is similar to the first, but occurs ahead of the events in the film. In about $4 \%$ of voxels, the correlation is greater when the fourth segment voxel time series is time shifted toward the future, an effect, which is very strong at 1 second but widely present even at three seconds ahead of the expected stimulus event. These voxels are found throughout the sensory cortices, but not in other regions. Sensory response to the familiar movie stimulus is accordingly anticipatory and predictive, occurring ahead of the actual stimulus event.

\title{
Keywords
}

Prediction, fMRI, adult, secs-mins, phenomenology, protention

\section{Interaction of Temporal Predictions across Cues, Durations, and Behavioral Systems}

\author{
Matthew S. Matell \\ Department of Psychology, Villanova University, USA
}

Identifying the mechanisms underlying temporal prediction can be facilitated by examining how different temporal expectations influence one another. We have seen averaging of temporal expectations, shared shifts in temporal expectation, and the transfer of temporal information from Pavlovian to instrumental behavior. In the first situation, rats are trained using the peak procedure that two different cues signal two different durations (e.g., tone $=10 \mathrm{~s}$ and light $=20 \mathrm{~s}$ ). At test, presentation of the tone + light compound results in a scalar peak function at an intermediate duration (i.e., 16s), suggesting that rats are averaging their temporal memories under conditions of conflict (Swanton et al., 2009). This averaging behavior reflects the relative reliability of each cue in predicting reinforcement (Matell \& Kurti, 2014), and can be blocked by administration of a serotonergic 1a antagonist at test, suggesting that averaging occurs at retrieval. In the second situation, rats are again trained that two different cues predict two different durations (i.e., tone $=8 \mathrm{~s}$ and light $=16 \mathrm{~s}$ ). After discriminable temporal control is established, we shifted the duration associated with one of the cues (e.g., 16s light $=32 \mathrm{~s}$ ). Testing the response to the other cue demonstrates a shift in the expectation in the same direction (i.e., 8 s tone $=12 \mathrm{~s}$; De Corte et al., submitted). This common shift results from alteration in the reinforcement time of 
the first cue, rather than alteration in the reinforcement rate. In the third situation, rats are trained with a Pavlovian peak procedure that a conditioned stimulus (CS) cue predicts an unconditioned food reward (US) at a specific time. After subsequent training on a variable interval procedure, presentation of the CS modifies the rate of operant responding in a temporally specific manner, thereby indicating that that temporal information is transferred from Pavlovian to operant behavioral systems.

\title{
References
}

Swanton, D. N., Gooch, C. M., \& Matell, M. S. (2009). Averaging of temporal memories by rats. J. Exp. Psychol. Anim. Behav. Process, 35, 434-439.

Matell, M. S., \& Kurti, A. N. (2014). Reinforcement probability modulates temporal memory selection and integration processes. Acta Psychol., 147, 80-91.

De Corte, B. J., Della Valle, R. B., \& Matell, M. S. (submitted). 'The times they are a-changin': Temporal covariance and the common cause hypothesis.

\section{Stimulus and Temporal Control of Behavior}

\author{
Marcelo S. Caetano \\ Federal University of ABC (UFABC), Brazil
}

When trained with multiple fixed intervals (FIs), humans and other animals are able to predict the approximate time of an event and learn to discriminate different time intervals signaled by different environmental stimuli. After some training, stimulus- controlled performance emerges, which is illustrated by an earlier increase in responding for stimuli that signal shorter FIs, as compared with stimuli that signal longer FIs. However, previous work from our group has shown that, under certain conditions, stimulus control does not emerge even after hundreds of trials of training. Specifically, when signaled FIs are trained in a blocked design, performance seems to be controlled by the temporal regularity in the task, even though the stimuli still reliably signal the different FIs. Importantly, this counterintuitive result is only observed when single-trial analyses are performed, as averaged data seem to mask the effect of training design. That competition for behavior control between temporal cues and environmental stimuli can be interpreted as an instance of overshadowing, in which the temporal cues overshadow the stimuli. In this talk, I will present results from several different experiments in humans (e.g., Guilhardi et al., 2010; Labliuk et al., 2015) and rats (e.g., Caetano et al., 2012), including recent unpublished data from our lab, which describe the conditions under which stimulus- or temporallycontrolled performance is observed, and show that temporal cues seem to always prevail over external cues in the competition for behavior control. The implication of those results to training designs and current models of timing will be briefly discussed.

\section{Keywords}

Duration, behavior, humans, rats, secs-mins, stimulus control 


\title{
References
}

Caetano, M. S., Guilhardi, P., \& Church, R. M. (2012). Stimulus control in multiple temporal discriminations. Learn. Behav., 40, 520-529.

Guilhardi, P., Menez, M., Caetano, M. S., \& Church, R. M. (2011). The effect of stimulus discriminability on strategies for learning multiple temporal discriminations. Behav. Processes, 84, 476-483.

Labliuk, T. P., Guilhardi, P., Cravo, A. M., Church, R. M., \& Caetano, M. S. (2015). Determinants of temporal or stimulus control in humans and rats. Int. J. Comp. Psychol., 28, $1-13$.

\section{Associate Memory of Elapsed Time: Behavior, Brain Oscillations and Hippocampal Activity}

\author{
Vincent van de Ven, Julia Lifanov, Oana Iosif, Sarah Kochs, \\ Fren Smulders and Peter de Weerd \\ Department of Cognitive Neuroscience, Faculty of Psychology and Neuroscience, \\ Maastricht University, The Netherlands
}

The extent to which time duration is represented in associate memory remains underinvestigated. We designed a time paired associate task (TPAT) in which participants implicitly learnt cue-time-target associations between cue-target pairs and specific cue-target intervals ranging from 500 to $2000 \mathrm{msec}$ (van de Ven et al., 2017). Importantly, participants only judged whether a cue and probe item were part of the same pair, while making no explicit judgment about time. During learning, some cue- target pairs became associated to a short interval while others became associated to a long interval. During subsequent memory testing, cue-target pairs were shown with both the short and long intervals. Participants $(\mathrm{N}=41)$ showed increased accuracy of identifying matching cue-target pairs if the time interval during testing matched the implicitly learnt interval. A control experiment showed that participants had no explicit knowledge about the time associations. In subsequent neuroimaging experiments we investigated the neural correlates of TPAT memory performance. Using ultra-high field magnetic resonance imaging (UHF-MRI) study at 7 Tesla $(\mathrm{N}=6+)$ we found less hippocampal activity when time intervals during test trials matched the learnt interval, compared to when they did not match. Further, in an electroencephalography (EEG) study $(\mathrm{N}=16)$ we found decreased Theta oscillation power (centered at $6 \mathrm{~Hz}$ ) at midline occipital/parietal scalp locations for the same comparison of trial types. These findings are in line with the role of hippocampus and Theta oscillations in associate memory (Buzsáki, 2006) and suggest that the same mechanisms also play a role in representing time in memory (Ranganath \& Hsieh, 2016). Mismatch between presented and expected associate memory of time may change hippocampal activity and cortical Theta oscillations, possibly through a common neural source. We suggest that cue-dependent retrieval of time in associate memory could perhaps serve as a mechanism for prospective coding of expected visual spatiotemporal events. 


\title{
Keywords
}

Associate time memory, prediction, ultra-high-field fMRI, EEG, adult, 100s of ms-secs

\section{References}

Buzsáki, G. (2006). Rhythms of the brain. Oxford: Oxford University Press.

Ranganath, C., \& Hsieh, L. T. (2016). The hippocampus: A special place for time. Ann. N. Y. Acad. Sci., 1369, 93-110.

van de Ven, V., Kochs, S., Smulders, F., \& De Weerd, P. (2017). Learnt interval time facilitates associate memory retrieval. Learn. Mem.

\section{Interval Timing in Aging Mice}

\section{Ezgi Gür1, Yalçın Akın Duyan', Sertan Arkan², Ayşe Karson² and Fuat Balci ${ }^{1}$}

\author{
${ }^{1}$ Koç University, Department of Psychology, Research Center for Translational Medicine, \\ Istanbul, Turkey \\ ${ }^{2}$ Kocaeli University, Faculty of Medicine, Department of Physiology, Kocaeli, Turkey
}

The aim of this study was to investigate how interval timing behavior of mice as assessed in the dual peak procedure is altered by aging. To this end, 38 male mice (C57BL/6) at different ages (4, 10 and 18 months old) were tested in the dual peak procedure with $25 \mathrm{~s}$ and $50 \mathrm{~s}$ target intervals. Start, stop, middle times, and spread derived from singletrial analysis were compared between age groups for steady-state performance. Correlations between start-stop, start-spread, and middle-spread were examined to estimate the contribution of different sources of timing variability in different age groups (Gibbon \& Church, 1992). Preliminary analyses showed that aged mice acquired temporally controlled response terminations later than young and adult mice. Although no differences were observed between the start times of different age groups, the stop times of old mice were significantly later than the stop times of young and adult mice at steady state. Middle times were longer than the short interval in all age groups, while it was also longer than the long interval in aged mice. Positive start-stop, negative start-spread, and positive middlespread correlations pointed at the contribution of both clock/memory and decision processes to timing variability in all age groups. Although the obtained correlation patterns did not point at altered contributions of different sources of timing variability across age groups, the prominent differences in stop times are suggestive of the failure of behavioral inhibition in old mice. We are currently investigating the relationship between these behavioral observations and the neurobiological markers that relate to the dopaminergic and acetylcholinergic function.

\section{Keywords}

Duration, behavior, aging, animal (C57BL/6 mice), secs-mins 


\title{
Reference
}

Gibbon, J., \& Church, R. M. (1992). Comparison of variance and covariance patterns in parallel and serial theories of timing.J. Exp. Anal. Behav., 57, 393-406.

\section{Temporal Estimation of Familiar Actions in Children and Adults}

\author{
Anne-Claire Rattat ${ }^{1}$ and Valérie Tartas ${ }^{2}$ \\ ${ }^{1}$ Laboratoire Sciences de la Cognition, Technologie, Ergonomie (SCoTE) EA 7420, \\ Université Fédérale de Toulouse Midi-Pyrénées, INU Champollion, Albi, France \\ ${ }^{2}$ Laboratoire Cognition, Langues, Langage, Ergonomie (CLLE) UMR 5263, Université \\ Fédérale de Toulouse Midi-Pyrénées, Toulouse, France
}

Duration of event is an important aspect of temporal knowledge that children experience from a very early age. Estimating the duration of an action often requires that we reference our own knowledge about the duration of similar actions stored in long-term memory. As a consequence, the ability to encode and maintain actions' durations in long-term memory is a keystone of time estimation. Until now, studies on memory for durations have been conducted mainly on the explicit aspect of arbitrary duration (e.g., 5-s tones) estimation and mainly with adults, and less is known about its developmental aspects (Rattat \& Droit-Volet, 2007). A unique study conducted by Friedman (1990) showed that children as young as 3 years of age are able to use a scale to make relative duration judgments about daily events. The present study aims at examining memory for familiar durations by using a developmental approach, by using two different but complementary methods. In a first experiment, we examine whether or not children aged 3-, 5- and 8-years old and adults are able to categorize familiar actions according to their duration, by using a forcedchoice categorization task. The results showed that with age increasing, the percentage of accurate temporal categorization increased while that of temporal categorization errors decreased. In a second experiment, 5- and 8-years old and adults have to produce the duration of familiar actions, this method being more efficient for studying memory for familiar durations in adults. The results showed that both the 8-year-olds and the adults produced longer durations for the long than for the short actions, whereas no difference was observed in the younger children. These findings are discussed in the light of event representation theory and the role of experience in temporal cognition.

\section{Keywords}

Durations, children, behavior, secs-mins

\section{References}

Friedman, W. J. (1990). Children's representation of the pattern of daily activities. Child Dev., 61, 1399-1412.

Rattat, A.-C., \& Droit-Volet, S. (2007). Implicit long-term memory for duration in young children. Eur.J. Cogn. Psychol., 19, 271-285. 


\title{
Selective Impairment of Temporal versus Spatial Predictive Coding in Schizophrenia
}

\author{
Valentina Ciullo $^{1,2}$, Federica Piras ${ }^{1}$, Gianfranco Spalletta ${ }^{1,3}$ \\ and Jennifer T. Coull ${ }^{4}$ \\ ${ }^{1}$ Neuropsychiatry Laboratory, Department of Clinical and Behavioral Neurology, \\ IRCCS Santa Lucia Foundation, Rome, Italy \\ ${ }^{2}$ Department of Neurosciences, Psychology, Drug Research and Child Health, \\ University of Florence, Italy \\ ${ }^{3}$ Menninger Department of Psychiatry and Behavioral Sciences, \\ Baylor College of Medicine, Houston, Texas, USA \\ ${ }^{4}$ Laboratoire des Neurosciences Cognitives, Aix-Marseille University, \\ France \& Centre National de la Recherche Scientifique, Paris, France
}

Timing disturbances have been proposed as an endophenotypic marker for neuropsychiatric disorders within the psychotic-affective spectrum. However, in- depth investigations of disorder-specific timing alterations are still scarce. As failures in predictive coding have been consistently observed in schizophrenia and possibly linked to the core symptom pathology, we investigated the behavioural benefits of predictive coding in the temporal versus spatial domain in 30 schizophrenic (SZ) and 30 bipolar (BD) patients, compared to matched healthy controls (HC). In an attentional orienting task, participants detected visual targets appearing at one of three locations after one of three intervals. Expectations about stimulus location (spatial condition) or onset (temporal condition) were conveyed by valid or invalid symbolic cues. Results indicated that SZ patients were able to make use of sensory information to form temporal predictions implicitly as their performance was influenced by foreperiod effects. By contrast, patients did not benefit from symbolic predictive cues (valid vs. neutral) in either the temporal or spatial dimension. Nevertheless, they were perturbed by spatially unexpected (invalid) events, though not temporally unexpected ones. Taken together, results confirm that SZ rely more upon sensory evidence to make predictions than top-down priors, and that this is particularly the case in the temporal domain. We also measured performance on temporal and colour discrimination tasks that were matched for working memory (WM) demands. Although temporal discrimination was impaired in SZ, confirming prior findings, colour discrimination was equally impaired, suggesting duration estimation impairments in SZ may be underpinned by WM dysfunction. BD patients showed no significant problems in timing compared to $\mathrm{HC}$, in either the orienting or discrimination paradigms. Results therefore also challenge the theory of a common biological phenotype for SZ and BD, and provide evidence that predictive timing may serve as a model system for differentiating the specific pathophysiology of these neuropsychiatric disorders.

\section{Keywords}

Prediction, duration, neuropsychology, schizophrenic patients, bipolar patients, 100s of ms-secs 


\title{
Spared Synchronization to the Beat of Music in the Presence of Poor Beat Perception
}

\author{
Valentin Bégel ${ }^{1,2}$, Charles-Etienne Benoit ${ }^{1,3}$, Angel Correa ${ }^{4,5}$, \\ Diana Cutanda ${ }^{6}$, Sonja A. Kotz ${ }^{7,8}$ and Simone Dalla Bella ${ }^{1,9,10}$
}

${ }^{1}$ EuroMov, Montpellier University, Montpellier, France

${ }^{2}$ NaturalPad, SAS, Montpellier, France

${ }^{3}$ Cognition and Actions Laboratory, Institute of Neuroscience, Université Catholique de Louvain, 1200 Brussels, Belgium

${ }^{4}$ Centro de Investigacion Mente, Cerebro y Comportamiento, University of Granada, Spain

${ }^{5}$ Departamento de Psicologia Experimental, University of Granada, Spain

${ }^{6}$ Centro de Investigacion Mente, Cerebro y Comportamiento, University of Granada, Spain

${ }^{7}$ Maastricht University, Faculty of Psychology \& Neuroscience, Dept. of Neuropsychology

\& Psychopharmacology, Maastricht, The Netherlands

${ }^{8}$ Max Planck Institute for Human Cognitive and Brain Sciences,

Dept. of Neuropsychology, Leipzig, Germany

${ }^{9}$ Institut Universitaire de France (IUF), Paris, France

${ }^{10}$ International Laboratory for Brain, Music, and Sound Research (BRAMS), Montreal,

Canada

Moving to the beat of music is widespread in humans. The majority, musicians and nonmusicians alike, can synchronize to the beat, for example by finger tapping or body swaying. Yet, some individuals encounter major difficulties in performing this task (poor synchronizers), a phenomenon initially ascribed to poor beat perception (i.e., beat deafness). More recently, however, it has been shown that poor synchronization can co-exist with unimpaired beat perception, thus pointing to a separation between beat perception and synchronization. Here we report the opposite dissociation (poor perception in the presence of spared synchronization) in two cases with beat deafness (L.A. and L.C). In the first experiment, participants' rhythmic skills were assessed with the Battery for The Assessment of Auditory Sensorimotor and Timing Abilities (BAASTA), which includes both perceptual and sensorimotor tasks, and compared to that of a control group. L.A and L.C performed poorly on rhythm perception tasks, such as detecting durational shifts in a regular sequence. Yet, they could tap consistently to the beat of the same stimuli. In the second experiment, we tested whether the aforementioned perceptual deficit in L.A. and L.C. extend to an implicit timing task. In this task the regular temporal pattern of isochronous auditory stimuli aids in performing a non-temporal tasks (i.e., responding as fast as possible to a different target pitch after a sequence of standard tones). L.A. and L.C's performance in the implicit timing task was comparable to that of controls. Altogether, the finding that synchronization to a beat can occur even in the presence of poor perception suggests that timing perception and action may dissociate at an explicit level. Spared 
implicit timing mechanisms might afford unimpaired synchronization to the beat in some beat-deaf participants.

\title{
Keywords
}

Rhythm, music, neuropsychology, adult, 100s of ms-secs

\section{Musical Rhythmic Training and Conversational Temporal Skills in Children with Deafness}

\section{Céline Hidalgo ${ }^{1,2}$, Simone Falk ${ }^{3}$, Noël Nguyen ${ }^{1}$ and Daniele Schön ${ }^{2}$}

\author{
${ }^{1}$ Aix Marseille Univ, CNRS, LPL, Aix-en Provence, France \\ ${ }^{2}$ Aix Marseille Univ, INSERM, INS, Inst Neurosci Syst, Marseille, France \\ ${ }^{3}$ Laboratoire Phonétique et Phonologie, UMR 7018, CNRS/Université Sorbonne Nouvelle \\ Paris-3, France
}

The smoothness of verbal exchanges during conversation depends on rhythmic coordination between interlocutors (Keller et al., 2014). The neuronal synchronization of the listener's sensory-motor system onto the speaker's speech rhythm gives the capacity to the listener to develop temporal predictions and anticipate speech turns. Cochlear implanted (CI) children show great variability in their linguistic development (Castellanos et al., 2015). A study has already shown the benefits of a rhythmic priming on their speech perception during a sentences repetition task (Cason et al., 2015) but no study has addressed the effect of a rhythmic training on temporal predictions at the conversational level. In three studies, we measure the temporal prediction skills of normal-hearing $(\mathrm{NH})$ children and CI children during verbal interaction and the benefits of a rhythmic training versus a usual auditory training in CI children. The first study, in which children name pictures in alternation with a virtual partner, shows that the rhythmic training makes IC children sensitive to a temporal structure within the verbal exchange. Using the same type of paradigm combined with a measurement of MMN, a second study shows that NH children detect temporal deviations in the regularity of the verbal exchange. Moreover, NH children converge spontaneously towards their interlocutor on temporal parameters such as speech rate. We also demonstrate an effect of rhythmic training compared to auditory training at an electrophysiological and behavioural levels in CI children. In a third study, we assess differences between $\mathrm{CI}$ and $\mathrm{NH}$ children in anticipating turns of conversational agents during a dialog as well as the extent to which a rhythmic training can possibly enhance these temporal prediction abilities in CI children.

\section{Keywords}

Prediction, music, language, electrophysiology, patients (children with deafness), 100s of ms secs 


\title{
References
}

Cason, N., Hidalgo, C., Isoard, F., Roman, S., \& Schön, D. (2015). Rhythmic priming enhances speech production abilities: Evidence from prelingually deaf children. $\mathrm{Neu}$ ropsychology, 29, 102-107.

Castellanos, I., Pisoni, D. B., Kronenberger, W. G., \& Beer, J. (2015). Neurocognitive function in deaf children with cochlear implants: Early development and long-term outcomes. In: The Oxford Handbook of Deaf Studies and Language: Policy, Practice, and Research (pp. 264-275).

Keller, P. E., Novembre, G., \& Hove, M. J. (2014). Rhythm in joint action: psychological and neurophysiological mechanisms for real-time interpersonal coordination. Philos. Trans. R. Soc. Lond. B Biol. Sci., 369, 20130394.

\section{The Role of Timing in Automatic Processing of Speech at Sub-Lexical and Lexical Levels}

\author{
Anita E. Wagner ${ }^{1,2}$ and Deniz Başkent ${ }^{1,2}$ \\ ${ }^{1}$ University of Groningen, University Medical Center Groningen, Department of \\ Otorhinolaryngology, The Netherlands \\ ${ }^{2}$ University of Groningen, Research School of Behavioral and Cognitive Neurosciences, \\ The Netherlands
}

Speech comprehension is automatic because the perceptual system enables instantaneous progression of information through various levels of analysis, from auditory object formation up to the integration of meaning within a conversation. How vital the timing of this progression is, becomes apparent when understanding speech becomes effortful, as it is for many profoundly deaf listeners, who perceive speech through a cochlear implant (CI). We study individual differences amongst CI users to investigate how the sensitivity to rhythmic structure in speech contributes to the automatic and effortless processing of speech via a CI. For individual listeners we created profiles of speech processing fluency based on cortical and behavioral measures that capture speech processing at various stages. Twelve experienced CI users, and twelve age-matched normal-hearing ( $\mathrm{NH})$ controls are compared in a set of measures: (1) cortical processing, measured as cortico- acoustic coherence in EEG recordings when listening to continuous speech; (2) the time course of lexical access captured in gaze fixations in a visual world paradigm while resolving lexical ambiguities; (3) the mental effort involved while processing speech, as measured in pupil dilation. NH listeners show cortico-acoustic coherence in the delta $(>4 \mathrm{~Hz})$ and theta $(4-8 \mathrm{~Hz})$ ranges. They also consistently rely on rhythm while resolving ambiguities, and show targeted and short-lived increase in mental effort during lexical access. For the CI users, individual variation in the mental effort involved in processing speech was related to individual differences in reliance on rhythm to resolve lexical ambiguities. Also, while 
all CI users showed increased cortico-acoustic coherence in the theta range, only CI users whose time-course of lexical access was similar to $\mathrm{NH}$ listeners showed coherence also in the delta range. This confirms the functional role of rhythmic structure in speech processing, but suggest also that extraction of this structure is more an active than automatic process.

\title{
A Functioning Model of Human Time Perception
}

\author{
Warrick Roseboom ${ }^{1,2}$, Zafeirios Fountas ${ }^{3}$, Kyriacos Nikiforou ${ }^{3}$, David \\ Bhowmik $^{3}$, Murray Shanahan ${ }^{3}$ and Anil Seth ${ }^{1,2}$ \\ ${ }^{1}$ Department of Informatics, University of Sussex, Brighton, UK \\ ${ }^{2}$ Sackler Centre for Consciousness Science, University of Sussex, Brighton, UK \\ ${ }^{3}$ Department of Computing, Imperial College, London, UK
}

Despite being fundamental to human behaviour and experience, how the brain achieves perception of time remains unknown. The predominant account - the internal clock approach and its derivatives - suppose the existence of a pacemaker based on neural oscillations, and accumulation process(es) to monitor these oscillations. A variety of neural functions and regions have been suggested to underlie these components, including subcortical structures such as basal ganglia (striatum), and cortical areas including insula and posterior parietal cortex, but an inability to determine the precise neural substrates of a pacemaker may indicate that the approach is flawed. As the experience of time is distributed over time, some kind of accumulation or memory process is required, but a counter that internally tracks time - like a computer system-clock - may not be. To test whether an internal clock is necessary for time perception we devised a novel artificial neural network architecture, taking advantage of the power of deep convolutional neural networks that are functionally similar to basic human sensory processing. We estimated time in this system by accumulating salient changes in each layer of the network over successive inputs of natural video scenes. System estimates of physical durations between 1 and $64 \mathrm{~s}$ replicate qualitative aspects of human time perception including Weber's law/scalar property and response regression to the mean (Vierordt's law). When compared to human estimates for the exact same stimuli the system estimates are well matched, including the presence of biases in apparent duration depending on scene type (e.g., city street, countryside, or office scenes). These findings demonstrate that an internal clock is not necessary for human time perception, providing a new understanding of this central aspect of experience.

\section{Keywords}

Duration, modelling, behavior, simulated data, adult, 100ms-secs-mins 


\title{
Resolving the Dopamine Paradox in Interval Timing: How a Phasic Dopamine Release Can Reset the Clock, whereas Tonic Dopamine Fluctuations Alter Perceived Time
}

\author{
Hedderik van Rijn ${ }^{1}$, Pim Mostert ${ }^{2}$, Michael LeKander ${ }^{1}$, \\ Pedro A. Martinez-Mediano ${ }^{3}$ and Zafeirios Fountas ${ }^{3}$ \\ 1University of Groningen, The Netherlands \\ ${ }^{2}$ Donders Institute for Brain, Cognition and Behavior, The Netherlands \\ Imperial College, UK
}

Typically, interval timing models assume that the subjective perception of time commences by the resetting of an internal clock, irrespective of whether this internal clock is hypothesized to be implemented as an pacemaker, as oscillating neurons, or as other structures that provide a temporally regular signal. Whereas most models are agnostic about the underlying signal that results in the resetting of the internal clock, the Striatal Beat Frequency model, the most prominent neurobiological model of the clock stage, assumes that a phasic release of dopamine resets the phases of clusters of oscillators. Interestingly, dopamine has been shown to have more subtle effects as well: when tonic dopamine levels are pharmacologically manipulated, the subjective experience of time is affected. Although both effects have been reported in multiple studies using multiple paradigms, no explanation has been given that can explain this paradoxical finding that a phasic boost resets the clock, but tonic fluctuations affect the internal clock in subtle but consistent ways (i.e., higher tonic dopamine levels resulting in a faster running internal clock). Here we will present a computational neuroscience model, implemented in BrainStudio (http://brain-studio.org) and based on biologically plausible neuron models, that explains how a phasic dopamine boost can reset an internal clock, but tonic dopamine levels affect the speed by which subjective time passes. By combining this more detailed implementation of the Striatal Beat Frequency model with an integrated process model of interval timing (Taatgen, Van Rijn, \& Anderson, 2007; Van Rijn, Gu, \& Meck, 2014), we can explain how the beginning of an interval is triggered, and what neurobiological mechanism explains cognitive and pharmacological distortions on subjective time.

\section{References}

van Rijn, H., Gu, B., \& Meck, W. H. (2014). Dedicated clock / timing-circuit theories of time perception and timed performance. Adv. Exp. Med. Biol., 829, 75-99.

Taatgen, N. A., van Rijn, H., \& Anderson, J. R. (2007). An integrated theory of prospective time interval estimation: The role of cognition, attention, and learning. Psychol. Rev., $114,577-598$. 


\title{
Excitation/Inhibition Balance in Cerebellum and Putamen Predict Individual Differences in Interval Timing
}

\author{
Devin B. Terhune ${ }^{1,2}$ \\ ${ }^{1}$ Department of Psychology, Goldsmiths, University of London, UK \\ ${ }^{2}$ Department of Experimental Psychology, University of Oxford, UK
}

There is accumulating evidence that timing of different interval ranges is subserved by distinct and overlapping mechanisms. Previous research has implicated a broad network of regions including cerebellum, striatum, and pre-supplementary motor area (preSMA) in different facets of timing of subsecond and suprasecond intervals but the role of neurochemicals in these regions in inter-individual differences in interval timing is poorly understood. In particular, an open question is whether cortical excitation is associated with temporal dilation or contraction and whether this effect is specific to particular brain regions and temporal intervals. In order to investigate these questions, this study used magnetic resonance spectroscopy (MRS) to measure resting state concentrations of the neurochemicals GABA and glutamate in three regions of interest in healthy adults $(\mathrm{N}=28)$ :anterior right putamen, right posterior cerebellum (lobule V/VI), and right pre-SMA. A fourth control region was superior postcentral gyrus, along the midline, which is the somatosensory area corresponding to the representation of the foot. Participants independently completed temporal bisection tasks spanning three interval ranges: 104-416 ms (short), 780-1404 ms (middle), and 2236-3484 ms (long). The excitation/inhibition balance (glutamate/GABA) in putamen was positively associated with bisection points (BPs) for middle and long, but not short, interval ranges. By contrast, the cerebellum excitation/inhibition balance was positively associated with BPs only for the middle interval range. The cerebellum and putamen associations with middle-interval BPs were inter-dependent, suggesting that individual differences may relate to putamen-cerebellar connectivity. BPs for all three interval ranges were not reliably associated with the excitation/inhibition balance in pre-SMA or the control region. These results suggest that excitation/inhibition balance in cerebellum and putamen is associated with temporal overestimation in the subsecond to suprasecond range and point to distinct and overlapping roles for these regions in interval timing.

Keywords

100s of ms-secs, adult, behaviour, duration, MRI

\section{Dopaminergic Modulation of Time Perception: A Biophysical Model}

Joachim Hass ${ }^{1,2}$ 


\author{
${ }^{1}$ Department of Theoretical Neuroscience, Bernstein-Center for Computational \\ Neuroscience, Central Institute of Mental Health, Medical Faculty Mannheim of \\ Heidelberg University, Germany \\ ${ }^{2}$ Faculty of Applied Psychology, SRH University Heidelberg, Germany
}

Pharmacological experiments in both animal and man show that the estimation of time is affected by dopamine (Buhusi \& Meck, 2005). Specifically, the duration of time intervals is underestimated if D2 dopamine receptors are inactivated. The mechanism for this phenomenon and its receptor specificity is unknown. To provide a possible explanation, we incorporate the currently known effects of D2 modulation on synaptic properties into a biophysical cortical model of time perception. The model is based on the slow increase of firing rates (Durstewitz, 2003) which is observed in cortical areas such as the prefrontal cortex: If firing rates increase monotonically over the range of several seconds, the rate at any point in time provides a code for the time elapsed since the start of the increase. We incorporate the effects of D2 in the prefrontal cortex, namely a decrease in both NMDA and GABA peak conductances. This modulation turns out to affect the slope of the rate in such a way that increasing D2 activation yields a steeper increase, and vice versa. Thus, blocking D2 receptors indeed yields a bias towards slowing the internal clock. Furthermore, we discuss the incorporation of this model into a recent data-driven network model of prefrontal cortex (Hass et al., 2016). Using this model, measures from electrophysiological recordings can be directly converted into distributions of neuron parameters. We have assessed the change of these distributions under dopaminergic modulation, which can now complement the synaptic effects described above and thus draw a more complete picture dopamine's effect on time perception within this model. More generally, we argue that successful reproduction of dopaminergic modulation can be used as a testbed to probe the biological validity of neurocomputational models of time perception.

\title{
Keywords
}

Dopamine, duration, modeling, simulated data, 100s of ms-secs

\section{References}

Buhusi, C. V. \& Meck, W. H. (2005). What makes us tick? Functional and neural mechanisms of interval timing. Nature Neurosci., 6, 755-765.

Durstewitz, D. (2003). Self-organizing neural integrator predicts interval times through climbing activity. J. Neurosci., 23, 5342-5353.

Hass, J., Hertäg, L., \& Durstewitz, D. (2016) A detailed data-driven network model of prefrontal cortex reproduces key features of in vivo activity. PLoS Comput. Biol., 12, e1004930. 


\title{
POSTERS
}

(in order of presentation)

\section{Time Perception in Long Term Memory}

Joffrey Derouet ${ }^{\mathbf{1}}$, Valérie Doyère ${ }^{\mathbf{2}}$ and Sylvie Droit-Volet ${ }^{\mathbf{1}}$

${ }^{1}$ University Clermont Auvergne, CNRS, Clermont-Ferrand, France

${ }^{2}$ Paris-Saclay Institute of Neuroscience, Neuro-PSI, CNRS-UMR 9197, Orsay, France

How long-term memory of durations is formed? The few studies in human adults observed a lengthening effect in the judgment of time, when the participants recalled a standard duration learned 24 hours before and when the memory consolidation process was disrupted by an interfering task shortly after learning. The present study examined whether this extension of time in long-term memory depends on the time scale of the memorized duration. We used a temporal generalization task in which participants had to learn and memorize a standard duration (either $600 \mathrm{~ms}, 4 \mathrm{~s}$ or $8 \mathrm{~s}$ ), and had to judge, 24 hours later, the similarity between the comparison durations and the standard duration. In addition, the participants of each standard duration group had to perform, or not, an interfering task 30 minutes after the learning phase, to examine the effect of the memory consolidation perturbation on time judgment. The results found a lengthening effect for all duration scales. The extension of time in long-term memory is therefore a robust phenomenon.

Keywords

Duration, memory, long-term memory, memory consolidation

\section{Executive Processes and Timing: Comparing Timing with and without Reference Memory}

\author{
Ruth S. Ogden', Rhiannon MacKenzie-Phelan' ${ }^{1}$, Catharine Mongtomery', \\ John E. Fisk ${ }^{2}$ and John H. Wearden ${ }^{3}$ \\ ${ }^{1}$ School of Natural Sciences and Psychology, Liverpool John Moores University, UK \\ ${ }^{2}$ School of Psychology, University of Central Lancashire, UK \\ ${ }^{3}$ School or Psychology, Keele University, UK
}

Temporal perception is influenced by executive function capacity (Droit-Volet et al., 2015; Ogden et al., 2014). However, performance on different temporal tasks is often associated with different executive functions. The current study examined whether using reference memory during a task influenced how task performance was association with executive 
resources. Participants completed temporal generalization and bisection tasks, in their normal versions involving reference memory, and episodic versions without reference memory. Each task had two levels of difficulty; easy and hard. Performance on these tasks were correlated with performance on measures of executive function; updating, inhibition, task switching, and access to semantic memory. Accuracy on the temporal generalization task was correlated with memory access, for all versions of the task, normal and episodic, and easy and more difficult. Updating correlated with accuracy only for the reference memory-based version of the task. Temporal bisection performance presented a different pattern of correlations. The bisection point was negatively correlated with inhibition scores, except for the easy episodic condition. The Weber ratio, considered a measure of temporal sensitivity was negatively correlated with memory access only in the hard episodic condition. Therefore, in spite of the fact that temporal generalization and bisection have been treated as conceptually similar, they appear to be based on different psychological processes. In addition, only temporal generalization showed different correlations for the normal and episodic conditions, perhaps suggesting that temporal bisection is not in fact based on the use of reference memory, or, that the reference memory load is so low that individual differences in memory do not influence performance, whatever procedure is used.

\title{
Keywords
}

Executive function, temporal generalization, temporal bisection, reference memory

\section{References}

Droit-Volet, S., Wearden, J. H., \& Zelanti, P. S. (2015). Cognitive abilities required in time judgment depending on the temporal tasks used: A comparison of children and adults. Q.J. Exp. Psychol., 68, 2216-2242.

Ogden, R. S., Wearden, J. H., \& Montgomery, C. (2014). The differential contribution of executive functions to temporal generalisation, reproduction and verbal estimation. Acta Psychol., 152, 84-94.

\section{Retrospective Time Reproductions Revealed a Large Memory for Modulating Music}

\author{
E. A. Firmino ${ }^{1,2}$, J. L. O. Bueno ${ }^{1}$ and C. L. Krumhansl ${ }^{2}$ \\ ${ }^{1}$ Department of Psychology, Cornell University, Ithaca, New York, USA \\ ${ }^{2}$ Department of Psychology, University of São Paulo, Ribeirão Preto, São Paulo, Brazil
}

There is a controversy in the literature on the temporal capacity of memory for music with keys and modulations (Farbood, 2016). A musical key is the sense of hierarchy of chords and a modulation is the passage from one key to another. We addressed that issue by observing how retrospective time reproductions are affected by three variables: (1) when in 
the chord sequence the modulation (e.g., $\mathrm{C}$ to $\mathrm{Gb}, 60 \mathrm{~s}$ ) occurred; (2) when in the modulating sequence (e.g., $\mathrm{C}$ to $\mathrm{Eb}$ to $\mathrm{Gb}, 60 \mathrm{~s}$ ) the key with the longest duration occurred; and (3) when the participant had to respond (e.g., 25, 50, or 100s of response delay) to different interkey distances (e.g., C to F, 20s, close; or C to Gb, 20s, distant). The interkey distance is roughly measured by the number of chords shared by the keys involved. Experiments 1 and 2 showed that when the longest keys were the first or second, the reproductions were shorter. These results indicate that the earlier long keys have more influence than the opposite, but earlier short keys still have a residual influence. Experiment 3 showed the farther the interkey distance, the shorter the reproduction. All these results confirm predictions of the Expected Development Fraction (EDF) Model of Firmino and Bueno (2008, 2016). However, Experiment 3 showed that, in addition to the interkey distance effect, when the response delay was $25 \mathrm{~s}$, the reproductions were larger than the longer response delays. We then propose an improved EDF Model with a dynamic musical memory that has long capacity in order to simulate encoding, maintenance, and retrieval throughout the stimulus-response period. The model also posits that remembered extra-musical information (e.g., verbal, visual, etc.) interferes with the musical information more in short response delays than in long ones.

\section{Keywords}

Time reproduction, retrospective paradigm, interkey distance, music, behaviour, simulated data

\section{References}

Farbood, M. M. (2016). Memory of a tonal center after modulation. Music Percept., 34, 71-93.

Firmino, E. A., \& Bueno, J. L. O. (2008). Tonal modulation and subjective time. Journal of New Music Research, 37, 275-297.

Firmino, E. A., \& Bueno, J. L. O. (2016). Interkey distances also shorten subjective time estimations in real modulating tonal music. Music Percept., 33, 613-630.

\section{The Effect of Physiological and Psychological Stress on Temporal Memory}

\section{Raquel Cocenas-Silva ${ }^{1,2}$, Sylvie Droit-Volet ${ }^{3}$ and Edilaine Gherardi-Donato ${ }^{2}$}

${ }^{1}$ Faculty of Philosophy, Sciences and Letters, Department of Psychology, University of São Paulo, Ribeirão Preto, Brazil

${ }^{2}$ School of Nursing, Department of Psychiatric Nursing and Human Sciences, University of São Paulo, Ribeirão Preto, Brazil

3Department of Psychology, University Clermont Auvergne, LAPSCO, CNRS UMR 6024, France 
This study investigated the effects of physiological and psychological stress on temporal memory among nurses. Fifty-three nurses from an emergency situation of a tertiary level university hospital - University of São Paulo participated in this study. The Job Stress Scale (JSS), the Perceived Stress Scale (PSS) and the Childhood Trauma Questionnaire (CTQ) were self-applied. Then, the participants performed a temporal generalization task with a retention delay of 24 hours between the learning of the standard duration (4s) and the test. The participants also had their blood collected to examine oxidative stress levels through nitric oxide in plasma. The results showed the effect of occupational stress (JSS) on the temporal variability. This effect suggested that the temporal judgment was more variable when the participants have high exposure to occupational stress compared to those who have low exposure. Indeed, there was a significant linear relationship between the variability of time judgment and the level of early life stress (CTQ). Therefore, the variability of time judgment increased with the early life stress level. However, there was a quadratic relationship between the proportion of accurate temporal responses and the perceived stress (PSS). This indicates that the proportion of accurate responses increased with the level of perceived stress until a certain threshold of perceived stress, but after this threshold the proportion of accurate responses decreased. Our results also showed a significant quadratic relation between the nitric oxide and the proportion of accurate temporal responses and the peak time. Low stress level is thus positive and improves memory for time, converging with the reactions triggered in the alarm stage of the General Adaptation Syndrome (SAG) of stress. However, in the more advanced stages of stress, physiological and psychological stress triggers damages for temporal memory affecting mostly the variability of time judgment.

\title{
Keywords
}

Duration, neuropsychology, adult, secs-mins, stress

\section{Evidence against Increased Temporal Resolution induced by Images that Give Impression of Danger}

\author{
Keishi Nomura ${ }^{1}$ and Yuko Yotsumoto ${ }^{2}$ \\ ${ }^{1}$ Department of Integrated Sciences, The University of Tokyo, Tokyo, Japan \\ ${ }^{2}$ Department of Life Sciences, The University of Tokyo, Tokyo, Japan
}

Some people anecdotally report having experiences of time slowing down when they are suddenly exposed to a life-threatening event, but whether this reflects an enhanced temporal resolution of real-time processing is still under debate. Recently, Kobayashi and Ichikawa (2016) reported that observers detect briefly presented dangerous images with high accuracy and further concluded that the emotion induced by such images improves the observer's temporal resolution. In our study, the image analyses that we conducted on the pictures used in their experiments revealed that some image features were not 
controlled in their study (Experiment 1a). We also examined whether the results of Kobayashi and Ichikawa (2016) could be replicated (Experiment 1b). In the experiment, a color image was presented for a certain duration followed by a mask. In $75 \%$ of the trials, the color image was briefly switched to the same image in monochrome and the participants were asked to answer whether they perceived the monochrome image. We observed that the detection accuracy increased when the participants observed images with safe impressions instead of dangerous impressions, and that these detection accuracies correlated moderately with the mean saturations of the color images. In Experiment 2a, the dangerous impressions of the images were reduced while retaining their image statistics using a texture synthesis algorithm proposed by Portilla and Simoncelli (2000), and even after doing this, the results in this experiment were similar to those of experiment $1 \mathrm{~b}$. In Experiment $2 \mathrm{~b}$, we exchanged the color characteristics of both the image groups with each other while retaining their impressions of danger, and then observed that the results reversed. These findings suggest that the previously reported increased temporal resolution while viewing highly emotional images, is due to the confounding effect of basic image features, especially saturation, rather than the emotion induced by such images.

\title{
Keywords
}

Duration, behavior, adult, 10s-100s of ms

\section{References}

Kobayashi, M., \& Ichikawa, M. (2016). Emotions evoked by viewing pictures may affect temporal aspects of visual processing. Jpn. Psychol. Res., 58, 273-283.

Portilla, J., \& Simoncelli, E. P. (2000). A parametric texture model based on joint statistics of complex wavelet coefficients. Int. J. Comput. Vis., 40, 49-71.

\section{Climbing and Timing: Attentional Biases Mediate the Affect-Time Perception Binding in a High Risk Sport}

\author{
Judit Castellà, Cristina Cuello and Antoni Sanz \\ Department of Basic, Developmental, and Educational Psychology Universitat \\ Autònoma de Barcelona, Spain
}

Two field studies were conducted in a Via Ferrata in order to explore how affective response influences time perception during an arousing activity in a real life setting (passing through a $69 \mathrm{~m}$ long, $20 \mathrm{~m}$ high, two-rope bridge). In study $1(\mathrm{n}=61)$ two questionnaires were administered (1) at the end point of the bridge (high arousing condition), and (2) close to the end of the Via Ferrata (low arousing condition). Participants judged their affective state (arousal, valence, and dominance) and provided retrospective (duration estimation and passage of time judgments) and prospective (one-minute production task) temporal judgments. The results showed that real performance mediated the relationship between affect and retrospective time perception measures, with the exception 
of dominance, which directly influenced passage of time judgments. Regarding prospective measures, an increase in arousal led to shorter temporal productions. Study $2(n=96)$ was aimed at assessing whether the natural affective responses elicited by passing through the bridge induced attentional biases and this, in turn, affected time perception. Besides affective state and time perception, selective attention was assessed through a seven-item questionnaire. Overall, non-linear functions best predicted the relationships between affect, attention, time perception, and task performance. Also, a causal pathway affect $\rightarrow$ interoceptive attention $\rightarrow$ time perception was found. The results of the two studies are discussed in terms of the emotional factors underlying time perception in ecological contexts.

\title{
Keywords
}

Duration, behavior, adults, secs-mins, affective response, selective attention

\section{The Effect of Fearful Faces on Timing Mechanisms of the Brain}

\author{
Emre H. Kale ${ }^{1}$, Sertaç Üstün ${ }^{2}$ and Metehan Çiçek ${ }^{1,2}$ \\ ${ }^{1}$ Brain Research Center, University of Ankara, Ankara, Turkey \\ ${ }^{2}$ Dep. of Physiology, School of Medicine, University of Ankara, Ankara, Turkey
}

In this study effect of fear perception on the time perception is examined using fMRI. Participants performed a time discrimination task (TD) and face recognition task (FR) during the scans. In each trial two consecutive face pictures were presented. A cue stimulus in the beginning of the trial informed the participant which type of task, she/he will perform in that trial. If the cue is an equal sign participant evaluated whether the consecutive face pictures (Ekman, 1999) belong to the same person (FR); if the cue is a sandwatch the participant evaluated the duration of the faces and decided which picture's presentation lasted shorter (TD). Using $2 \times 2$ ANOVA [Task (TD/FR) $\times$ Emotion (Fearful/Neutral Faces)] design, we found that the positive main effect of task (TD > FR) included: Supplementary motor are, left anterior insula, left cerebellum, right inferior parietal cortex and right dorsolateral parietal cortex (FWE corrected, $\mathrm{p}<0.05$ ), which is in line with our previous work and the literature. No significant activations were shown for the positive main effect of the emotion (Fear $>$ Neutral), and the interaction. Interestingly, the negative main effect of the task (FR > TD) showed significant activation in bilateral amygdalae and bilateral fusiform face area (FFA). In the follow up ROI analysis, the percent signal change in the bilateral amygdala was showed to be higher in the FR task compared to the TD task. An explanation for the obtained result might be that activation of amygdalae were related to both fearful and neutral faces stimuli and that the FR task without differentiating fearful and neutral faces activated the amygdalae and fusiform face area (Davies-Thompson, 2012; Todorov, 2012). In conclusion, our results provide novel findings about the relationship 
between the time perception and emotional processing by supporting a differential effect of these brain functions on the amygdala activity.

\title{
Keywords
}

Duration, MRI, adult, 100s of ms-secs

\section{References}

Ekman, P. (1999) Facial expressions. Handbook of Cognition and Emotion, 16, 301-320.

Davies-Thompson, J., \& Andrews, T. J. (2012). Intra-and interhemispheric connectivity between face-selective regions in the human brain. J. Neurophysiol., 108, 3087-3095.

Power, J. D., Barnes, K. A., Snyder, A. Z., Schlaggar, B. L., \& Petersen, S. E. (2012). Spurious but systematic correlations in functional connectivity MRI networks arise from subject motion. Neuroimage, 59, 2142-2154.

Satterthwaite, T. D., Elliott, M. A., Gerraty, R. T., Ruparel, K., Loughead, J., Calkins, M. E., Eickhoff, S. B., Hakonarson, H., Gur, R. C., Gur, R. E., \& Wolf, D. H. (2013). An improved framework for confound regression and filtering for control of motion artifact in the preprocessing of resting-state functional connectivity data. Neuroimage, 64, 240-256.

Todorov, A. (2012). The role of the amygdala in face perception and evaluation. Motiv. Emot., 36, 16-26.

\section{Emotions and Brain Wave Activities: Determinants of Psychological Time}

\author{
Aman Choudhary ${ }^{1}$, Shashvat Shanker ${ }^{1}$ and Priyanka Kacker ${ }^{2}$ \\ ${ }^{1}$ Amity Institute of Behavioral and Allied Sciences, Amity University, Gurgaon (Manesar), \\ Haryana, India \\ ${ }^{2}$ Institute of Behavioral Science, Gujarat Forensic Sciences University, Gandhinagar, \\ Gujarat, India
}

In recent time a lot of focus has been made to study the time perception, how humans perceive and comprehend the passage of time. There are several factors involved in the time perception by human beings; they are auditory, visual, sensory etc. Various efforts are made to understand the origin and concept of psychological time by involving external as well as internal stimuli. Time perception depends on our intrinsic context, such as our emotional state, and on extrinsic context such as rhythmic activity around us. The emotions perceived theaters a major role in the perception of time, time is perceptualized in the right cortex and basal ganglia of the brain, and neurotransmitters (adrenaline and dopamine) play a vital role in time perception. As emotions have a major role in manipulating neurotransmitters, it could be said that emotions could increase or decrease the time of perception. In the current study, the focus is on the various emotions that were 
displayed to the subject, while brainwave activities were measured, and the time duration experienced psychologically. The sample consists of 30 individuals between the age group of 20-25 years from the technical and non-technical background. The brainwave activities are measured using electroencephalography (EEG) and analyzed according to the requirement of the present study.

\title{
Keywords
}

Emotions, time perception, electroencephalography, secs-mins, neuropsychology, young adults

\section{Induced Anxiety Leads to Underestimating Time}

\author{
Ioannis Sarigiannidis ${ }^{1,2}$, Christian Grillon ${ }^{2}$, Monique Ernst ${ }^{2}$, Jonathan \\ Roiser $^{1}$ and Oliver Robinson ${ }^{1}$ \\ ${ }^{1}$ Institute of Cognitive Neuroscience, University College London, UK \\ ${ }^{2}$ Affective Psychophysiology Laboratory, Section on Neurobiology of Fear and Anxiety, \\ NIMH, USA
}

Our subjective experience of time is an integral part of our mental life and is intimately linked to our emotional state (Droit-Volet \& Meck, 2007). Hedonic events are associated with quick passage of time but the picture is less clear for negative experiences. For example, during an anxiety- provoking interview time might fly but during traumatic events it might be reported that time froze. However, no study up to date has investigated how anxiety affects time perception. In two experiments we used threat of unpredictable shock to induce anxiety in healthy individuals performing two-alternative forced choice tasks, a subsecond (Experiment 1, $\mathrm{n}=25$ ) and a suprasecond (Experiment 2, $\mathrm{n}=25$ ) temporal bisection paradigm. Specifically, participants viewed stimuli that remained on the screen for different time intervals (Experiment 1: 300-700 ms, Experiment 2: 1400-1600 ms) and then decided whether their duration was "short" or "long" compared to anchor durations they had in mind. In line with our hypothesis, in both experiments, participants significantly underestimated time in the anxiety condition, as indicated by a rightward shift of the psychophysical function. Our effect seems to generalise using a wider range of time intervals (300-2800 ms, Experiment 3, $\mathrm{n}=12$, on-going recruitment) in both the visual and the auditory domain. Considering previous studies in which it was shown that fearful events lead to time overestimation (Tipples, 2011), our results support an attentional model of time perception for fear and anxiety: Fear is associated with increased allocation of attention to the to-be-timed event, while anxiety distracts from the timing processes, thus leading to time overestimation and underestimation respectively.

\section{Keywords}

Anxiety, duration, behaviour, adult, 100s of ms-secs 


\title{
References
}

Droit-Volet, S., \& Meck, W.H. (2007). How emotions colour our perception of time. Trends Cogn. Sci., 11, 504-513.

Tipples, J. (2011). When time stands still: Fear-specific modulation of temporal bias due to threat. Emotion, 11, 74-80.

\section{The Effect of Contemplative Rooms on the Perception of Time}

\author{
Sonja Ehret, Joana Post, Miriam Ruess and Roland Thomaschke \\ University of Freiburg, Germany
}

It was shown that the environment of a waiting room can influence the affect and the perception of the waiting time. The goal of the current study was to examine whether and how spending time in a contemplative room would change the perception of time compared to a less contemplative more typical waiting room. As contemplative states like mindfulness are known to affect time perception by the increase of self- awareness, there are rooms particularly constructed to create these states. In the present study, 24 participants had to spend 90 minutes of time in a church, as a contemplative room, and in a doctor's waiting room, as control condition, on two different days. All timers (e.g., clock, notebook, watch, smartphone) were removed from the rooms as well as from the participants. During waiting time the participants were instructed to stay inside the room while it was entirely left to them how to spend the time. In order to measure time perception, awareness of time, passage of time judgments, and duration of time were assessed. The results suggest that participants show a higher awareness of time while they were waiting in the church compared to the doctor's waiting room. The duration of time was underestimated in the waiting room condition whereas the estimation of duration in the church didn't significantly differ from the actual passed time. This suggests that the contemplative architecture of a room can modify the perception of time spend in that room. A possible explanation for this effect is that waiting in a contemplative room evokes a mindfulnessrelated state that enhances the awareness of the time. Further research should focus on the factors that determine this effect of contemplative rooms on the experience of time.

\section{Keywords}

Time perception, architecture, waiting, timing, contemplation 


\title{
Meditation-Induced Changes in Subjective Time Are Mediated by Heart-Rate Variability and Breathing Rate
}

\author{
Damisela Linares Gutierrez ${ }^{1}$, Sebastian Kübel ${ }^{1}$, Anne Giersch ${ }^{2,3}$, \\ Stefan Schmidt ${ }^{4,5}$, Karin Meissner ${ }^{6,7}$ and Marc Wittmann ${ }^{1}$ \\ ${ }^{1}$ Institute for Frontier Areas in Psychology and Mental Health, Freiburg, Germany \\ ${ }^{2}$ INSERM U1114, Strasbourg, France \\ ${ }^{3}$ FMTS, Psychiatry Department, University Hospital of Strasbourg, France \\ ${ }^{4}$ Department of Psychosomatic Medicine, University Medical Center Freiburg, Germany \\ ${ }^{5}$ Institute for Transcultural Health Studies, European University Viadrina, \\ Frankfurt/Oder, Germany \\ ${ }^{6}$ Institute of Medical Psychology, Ludwig-Maximilian University Munich, Germany \\ ${ }^{7}$ Division Integrative Health Promotion, University of Applied Sciences, Coburg,
}

Germany

Related to conceptualizations of a relationship between meditation, subjective time, and psychophysiology, we conducted a study probing for changes in temporal integration after meditation. We employed three psychophysical tasks related to perception of sequence in the milliseconds range, the integration of metronome beats, and the perception of alternations in the ambiguous Necker cube. The tasks were administered before and after the intervention. The 91 participants were experienced mindfulness meditators who in three separate 10 minute sessions either meditated by following a meditation session $(n=44)$ or listened to an audio play $(n=47)$, the three counterbalanced sessions conducted on three separate days. During the intervention heart-rate and breathing rate was recorded and compared to a resting-state condition. Applying path analyses, we found several mediating and moderating effects. Regarding subjective scales, those meditators who were less aware of the passage of time felt less emotional arousal. Regarding psychophysiology and switching of the Necker cube, breathing rate significantly mediated the effect of meditation across all participants, that is, the slower breathing during meditation the slower the switching of the two aspects in the Necker cube. Additionally, a moderator effect showed that trait-mindfulness leads to slower switching times in meditators. Regarding the metronome task, and over all frequencies meditation in more experienced meditators $(n>100$ hours) lead to a decrease of integration intervals. However, for the fastest (ISI $=0.3 \mathrm{~s}$ ) and slowest (ISI = $3 \mathrm{~s}$ ) metronome frequencies an opposite effect occurred: the larger RMSSD during meditation the larger the integration interval. Overall, these findings add evidence to meditation-induced changes in subjective time and the general notion of embodiment of mental functioning.

\section{Keywords}

Mindfulness meditation, temporal integration, Necker cube, metronome, sequencing 
Acknowledgements

This work was sponsored by the FUNDAÇÃO Bial, Portugal, through a grant to Marc Wittmann, Stefan Schmidt, and Karin Meissner: "Changes in subjective time as indication of increased mindfulness after meditation".

\title{
The Effect of Focused-Attention Meditation Dosage on Time Perception
}

\author{
Zaffie Cox and Dinkar Sharma \\ University of Kent, UK
}

\begin{abstract}
Kramer, Weger and Sharma (2013) have shown that mindfulness-based focused-attention meditation can alter time perception. Using the temporal-bisection task their study showed that an 8-minute meditation intervention led to an overestimate of time compared to a control group listening to an audio clip. The current study was designed to replicate this research and extend it by looking at the effect of different meditation dosages. The length of the meditation intervention was varied in which identical instructions were heard but the duration of the self-practice elements were of 20 seconds, 6 minutes or 12 minutes in three different groups. As in Kramer et al., our study used the same temporal-bisection task where probe durations were compared to "short" (400 ms) or "long" (1600 ms) standard durations. The temporal-bisection task was completed before and after the meditation session. Results show that longer meditation practice times led to participants displaying greater overestimations of time. This supports the previous research and suggests that meditation self-practice is related to these overestimations of time. These results could have implications for the application of mindfulness-based meditative interventions, both in their efficacy and in their duration, for both clinical and everyday participants.
\end{abstract}

\section{Keywords}

Focused-attention meditation, meditation duration, mindfulness, temporal bisection

\section{Reference}

Kramer, R., Weger, U., \& Sharma, D. (2013). The effect of mindfulness meditation on time perception. Conscious. Cogn., 22, 846-852.

\section{Time Estimation and Body Awareness}

\author{
Tereza Nekovarova ${ }^{1,2,4}$, Katerina Sedlakova ${ }^{1,2}$, Adela Beckova ${ }^{1,5}$, \\ Kristyna Maleninska ${ }^{1,3}$, Jan Rydlo ${ }^{1}$ and Jiri Horacek ${ }^{1,2}$ \\ ${ }^{1}$ National Institute of Mental Health, Klecany, Czech Republic
}


${ }^{2}$ Third Faculty of Medicine, Charles University in Prague, Prague, Czech Republic ${ }^{3}$ Department of Neurophysiology of Memory, Institute of Physiology Academy of Sciences of the Czech Republic v.vi.., Prague, Czech Republic

${ }^{4}$ Department of Zoology, Faculty of Natural Sciences, Charles University, Prague, Czech Republic

${ }^{5}$ Department of Psychology, Faculty of Arts, Charles University, Prague, Czech Republic

Time perception is a crucial cognitive domain forming and affecting our perception of reality. We assume that time perception may be based both on external information and perception of internal body signals, even if interoception may be an unconscious process. In our study we focus on individual differences in time estimation accuracy and interoceptive sensitivity. We study time estimation accuracy in healthy volunteers who complete timing tasks and correlate it with body consciousness. To measure time estimation, we use repeated reproduction of rhythm task (the participant is asked to maintain the given frequencies with 300 or $900 \mathrm{~ms}$ inter-response intervals, respectively). To assess interoceptive awareness we used heartbeat perception task (Tsakiris et al., 2011) and Body Consciousness Questionnaire. As self-representation and interoceptive awareness may be affected in some neuropsychiatric diseases (e.g., schizophrenia) understanding of relation between time estimation and body consciousness may contribute to deeper understanding of mechanisms of time perception and its disruptions.

\title{
Keywords
}

Rhytms, neuropsychology, adult, 100s of ms-secs

\section{Acknowledgements}

This study was funded by the project Nr. LO1611 with a financial support from the MEYS under the NPU I program; by projects $260388 /$ SVV/2017 and Progres Q 35; and by GACR grant 15-08577S.

\section{Reference}

Tsakiris, M., Tajadura-Jiménez, A., \& Costantini, M. (2011). Just a heartbeat away from one's body: Interoceptive sensitivity predicts malleability of body-representations. Proc. R. Soc. Lond., B, Biol. Sci., 278, 2470-2476.

\section{Effects of Delayed Auditory Feedback on Temporal Reproduction Task with Single Motor Response}

\author{
Keita Mitani ${ }^{1,2}$ and Makio Kashino ${ }^{1,3}$ \\ ${ }^{1}$ Tokyo Institute of Technology, Kanagawa, Japan \\ 2Japan Society for the Promotion of Science, Tokyo, Japan \\ ${ }^{3}$ NTT Corporation, Kanagawa, Japan
}


Previous studies have shown that delayed auditory feedback (DAF) disrupts sequential motor responses such as speech production, musical performances and sensorimotor synchronization. However, because these studies use a sequential motor task, it remains unclear whether DAF disrupts temporal reproduction that has a single motor response. In contrast to sequential motor tasks, such a single motor task with DAF does not require a concurrent process for motor planning, execution, and resolving of a temporal discrepancy between an action and its feedback. Here, we employed a temporal reproduction task with DAF and a single motor response. After participants listened to 3 equally spaced sequential tones with a base interval (lengths: 300, 500, and $700 \mathrm{~ms}$ ), they must then produce an isochronous timed feedback tone by pressing a button. An artificial delay (lengths: $0,100,200 \mathrm{~ms}$ ) between the button- press and its feedback tone was inserted. The results indicated that the average timing of feedback tones was later when increasing the delay, whereas the standard deviation was significantly larger for the 200-ms delay and comparable for the 0 - and 100-ms delays. In addition, to examine whether these DAF effects were dependent on the base interval lengths, we developed a linear regression model as a function of the delay lengths for each base interval for both the average and standard deviation. This analysis revealed that the DAF effect for the average was significantly stronger for the 300-ms base interval than the other base intervals, but for the standard deviation had no significant difference. In conclusion, we showed that the temporal accuracy and precision of a single motor response are affected differently by DAF. Moreover, we also showed that the magnitude of the DAF effect on the accuracy depends on the base interval length.

\title{
Keywords
}

Duration, multisensory, behavior, adult, 10s-100s of ms, 100s of ms-secs

\section{A Time Limit Decreases the Accuracy of the Sequential Lever-Press Performance in Rats}

\author{
Yayoi Sekiguchi ${ }^{1}$ and Toshimichi Hata ${ }^{2}$ \\ ${ }^{1}$ Graduate School of Psychology, Doshisha University, Japan \\ ${ }^{2}$ Faculty of Psychology, Doshisha University, Japan
}

We examined how a time limit affected rat's performance of a sequential lever-press task in an operant chamber. Each trial began with the presentation of a tone or light stimulus, followed by lever insertion. Rats were trained to press two levers alternately four times to obtain rewards. In the test phase, each stimulus signaled either a FREE or LIMITED condition. Under the LIMITED condition, a time limit was set for each rat, as the longest time needed to complete the four responses on the training days (about 7 seconds). Under the FREE condition, there was a 25 -second time limit. The duration of each stimulus was equal to the length of the time limit. All trials were classified as one of three types based on the rats' performance. When levers were pressed in the correct order, it was recorded as a "correct trial." When the responses were performed in the correct order, with at least one response resulting in a lever being touched rather than pressed, it was recorded as an "incomplete trial." If 
the responses were performed in the incorrect order, it was recorded as a "sequence-error trial." As a result, under the LIMITED condition, the correct trial rate in the test phase was significantly lower than that in the last days of the training. The decrease in the correct trial rate was accompanied by an increase in the incomplete trial rate. There was no difference in the sequence-error trial rate or in the reaction time between days or conditions. Additional analyses revealed that these effects were more evident in rats that performed slowly and accurately. Although there was no difference in the reaction time between conditions, the limited response time could result in reduced performance accuracy depending on the rats' behavioral tendencies, even if the limitation was minimal.

Keywords

Duration, behavior, rats, secs-mins, time limit

\title{
Pre-Feeding and Timing: Temporal and Motivational Mechanisms of Discrimination
}

\author{
Oscar Zamora-Arevalo, Mayra P. Cruz-Flores and Mario Perez-Calzada
}

Facultad de Psicología, Universidad Nacional Autónoma de México, Mexico

The current study examined this issue further by training and testing rats under different motivational conditions. Ten rats were exposed to a temporal bisection task in four different pairs of durations (short-long). Once the discrimination criterion was met, then tested with intermediate durations with two conditions no pre-feeding and pre feeding with 7.5 $\mathrm{g}$ of pellets in home cages 40 minutes before these generalization sessions. Results suggest that pre feeding changes sigmoidal functions in any form, not systematically, but it does in comparison to baseline. The findings are discussed in terms of psychophysical parameters obtained on the baseline, according to the parameters obtained with pre-feeding manipulations.

\section{Keywords}

Timing retrospective, perception, temporal bisection, pre-feeding, psychophysics, animals

\section{Time Is Money: Monetary-Reward Expectancy Distorts Perception of Duration}

\section{Mitra Taghizadeh Sarabi ${ }^{1}$, Hiroomi Kuninori ${ }^{1}$, Ryuta Aoki ${ }^{2}$ and Kiyoshi Nakahara ${ }^{1,2}$}

${ }^{1}$ School of Information, Kochi University of Technology, Japan

${ }^{2}$ Research Center for Brain Communication, Kochi University of Technology, Japan 
A recent behavioural study indicated that reward expectancy could elongate subjective perception of temporal duration (Failing \& Theeuwes, 2016). However, it is still unclear whether this effect is caused by general increase of arousal level associated with reward expectancy, or by a specific mechanism involving reward expectancy itself. To address this issue, we applied monetary incentive delay (MID) paradigm to duration judgment. Twenty subjects initially learned reference temporal duration of $450 \mathrm{~ms}$. Then, in each trial, one of three monetary cues ( +50 yen, \pm 0 yen, and -50 yen) signaled positive (Pos), negative (Neg), and neutral (Neu) reward conditions respectively. After a delay period, subjects judged whether the duration of target stimulus (300, 350, 400, 450, 500, 550, or $600 \mathrm{~ms}$ ) was shorter or longer than the reference. Reward outcome was determined by task performance in each trial; subjects gained 50 yen for a hit response in Pos condition and lost 50 yen for an incorrect or miss response in Neg condition. Neu condition did not affect the reward outcome. We postulated that both Pos and Neg conditions can equally increase subjects' general arousal levels, but only Pos condition induces reward expectancy. We fitted a psychometric curve to probabilities that a subject reported each target lasted longer than the reference and calculated the mean point of subjective equality (PSE) to the reference for each condition. We found that PSE was significantly smaller in Pos condition than both in Neu and Neg conditions $(\mathrm{P}<0.05$, Wilcoxon signed-rank test), whereas no significant difference was found between Neg and Neu conditions. Thus, our results suggest that monetary-reward expectancy may be involved in a specific mechanism that alters the speed of the internal clock.

\title{
Keywords
}

Reward expectancy, arousal, duration, behavior, adult, 100s of ms-secs

\section{Reference}

Failing M., \& Theeuwes J. (2016). Reward alters the perception of time. Cognition, 148, 19-26.

\section{Neural Mechanisms Underlying Time Perception and Reward Prospect}

\author{
Nihal Apaydın', ${ }^{2}$, Emre H. Kale², Sertaç Üstün ${ }^{3}$, İpek Çelikağ ${ }^{3}$, \\ Bora Baskak $^{2,4}$, Halise Devrimci Özgüven ${ }^{2,4}$ and Metehan Çiçek ${ }^{2,3}$ \\ ${ }^{1}$ Dep. of Anatomy, School of Medicine, University of Ankara, Ankara, Turkey \\ ${ }^{2}$ Brain Research Center, University of Ankara, Ankara, Turkey \\ ${ }^{3}$ Dep. of Physiology, School of Medicine, University of Ankara, Ankara, Turkey \\ ${ }^{4}$ Dep. of Psychiatry, School of Medicine, University of Ankara, Ankara, Turkey
}

We sought to elucidate the neural processes that are shared and distinct between brain regions responsible for time perception and reward prospect with the present functional magnetic resonance imaging (fMRI) study. Participants were 19 healthy volunteers, all 
right handed. We employed a temporal attention task in which observers had to extrapolate the velocity of an occluded moving object in "reward" vs. "no-reward" sessions. After the period of invisible motion, the target reappeared and participants were asked to make perceptual judgments whether it reappeared earlier or later compared with its predicted velocity in "time perception" condition. There were also "control condition" trials in which participants judged about the color change of the stimuli after reappearing. The result of the group analysis revealed a significant main effect of time in the right intraparietal sulcus. A right dorsolateral prefrontal cortical activity was also approaching significance. The interaction of reward and time showed a posterior cingulate cortex (PCC) activity. The right frontoparietal activation result related to time perception was in line with our previous results (Sertaç et al., 2017). Our results suggest that reward prospect interact with time processing in the brain by mechanisms located in the frontoparietal cortical and most likely by subcortical brain regions proposed to be connected by PCC (Leech et al., 2012).

\title{
Keywords
}

Duration, MRI, adult, secs-mins

\section{References}

Leech, R., Braga, R., \& Sharp, D. J. (2012). Echoes of the brain within theposterior cingulate cortex. J. Neurosci., 32, 215-222.

Üstün, S., Kale, E. H., \& Çiçek, M. (2017). Neural networks for time perception and working memory. Front. Hum. Neurosci., 11, 83.

\section{Visual and Phonological Repetition, but not Semantic Repetition, Compresses Perceived Duration}

\author{
Zhuanghua Shi ${ }^{1}$ and Lina Jia ${ }^{2}$ \\ ${ }^{1}$ Department of Psychology, Ludwig-Maximilians-Universität München, Munich, \\ Germany \\ ${ }^{2}$ Department of Education, School of Humanities, Jiangnan University, Wuxi, China
}

Our prior experience heavily influences our subjective time (Shi, Church, \& Meck, 2013). One of such phenomena is repetition compression (Matthews \& Gheorghiu, 2016), that is, repeated stimuli are perceived shorter than novel stimuli. However, most of the studies on repetition compression used identical stimuli, leaving the question whether similar repetition effects could take place in phonological and semantic level repetition. Here we used Chinese characters to manipulate three levels of repetition in a duration discrimination task: the visual (e.g., 木 - 木 ), phonological (e.g., 目 /mu/- 木 /mu/), and semantic (e.g., 足 /zu/ - 脚 /jiao/) repetitions. We replicated earlier findings that repetition of visual identical characters shortened the apparent duration. The repetition compression was location independent. More interestingly, phonological repetition also caused the duration 
compression, but the effect was weaker than the visual repetition. However, we failed to observe duration compression during the semantic repetition. The results suggest that repetition compression is mediated by visual and phonological representation of a stimulus in an early stage in processing hierarchy. We argue that the degree of predictability determines the magnitude of the repetition compression effect, which is in line with the framework of predictive coding (Shi \& Burr, 2016).

\title{
Keywords
}

Duration judgment, repetition compression, Chinese characters

\section{References}

Matthews, W. J., \& Gheorghiu, A. I. (2016). Repetition, expectation, and the perception of time. Current Opinion in Behavioral Sciences, 8, 110-116.

Shi, Z., \& Burr, D. (2016). Predictive coding of multisensory timing. Current Opinion in Behavioral Sciences, 8, 200-206.

Shi, Z., Church, R. M., \& Meck, W. H. (2013). Bayesian optimization of time perception.

Trends Cogn. Sci., 17, 556-564.

\section{Emotional Content rather than Familiarity Could Be the Reason behind Distorted Time Perception in Addiction}

\author{
Lazaros Gonidis and Dinkar Sharma \\ School of Psychology, University of Kent, UK
}

Recent findings suggest that addiction related salient stimuli can lead to distorted time perception (Gonidis \& Sharma, 2017). However, one possible explanation could be that these differences, observed in salient but not in neutral stimuli, are due to stimuli familiarity and not due to salience. The aim of this experiment was to provide evidence that the distorted time perceptions are only due to salience and not due to familiarity or novelty. Participants learnt to associate non-words with salient and neutral stimuli. They then completed a Temporal Bisection task on the above learnt non-words and on new non-learnt non-words. Analysis showed that participants underestimated time for the non-words that were associated with salient stimuli compared to the non-words that were associated with neutral stimuli. There was also no difference in time perception between the learnt neutral non-words and the non-learnt ones. These findings suggest that addiction related stimuli can lead to distorted time perception due to the emotional content of the salient stimuli and not due to familiarity or novelty.

\section{Keywords}

Duration, behavior, adult, 100s of ms-secs, addiction, emotion, arousal 


\title{
Reference
}

Gonidis, L., \& Sharma, D. (2017). Internet and Facebook related images affect the perception of time.J. Appl. Soc. Psychol., 47, 224-231.

\section{The Effects of Gambling Related Stimuli on the Time Perception of Gamblers and Non-Gamblers}

\author{
Lazaros Gonidis and Dinkar Sharma \\ School of Psychology, University of Kent, UK
}

\begin{abstract}
Despite the increasing volume in the study of addiction there is still little research on addiction and time perception. However, recent evidence suggested that non- substance addiction related stimuli can affect the time perception of frequent users. More specifically Gonidis and Sharma (2017) found that Facebook users tend to underestimate time when exposed to Facebook related stimuli. The present study investigated whether similar distorted time perceptions can occur in another non- substance related addiction such as gambling. Two groups of University students, gamblers and non-gamblers, participated in a gambling modified Temporal Bisection task where they had to judge durations between 400 and 1600 ms. Results show that gamblers underestimated time when exposed to gambling related stimuli but not when exposed to neutral stimuli. No such differences were observed for the non- gambling group. These findings provide more evidence that non-substance addiction related stimuli can lead to distorted time perceptions as a result of attentional bias and/or arousal effects and also highlight the need to investigate more time perception in addictive behaviours.
\end{abstract}

\section{Keywords}

Duration, behavior, adult, 100s of ms-secs, addiction, emotion, arousal

\section{Reference}

Gonidis, L., \& Sharma, D. (2017). Internet and Facebook related images affect the perception of time.J. Appl. Soc. Psychol., 47, 224-231.

\section{Effect of Entrainment at Intermodulation Frequency on Perceived Duration}

\author{
Shogo Ito and Yuko Yotsumoto \\ Department of Life Sciences, University of Tokyo, Japan
}


The duration of a flickering visual stimulus is perceived longer than that of a steady stimulus. It has been shown that when the invisible flicker frequency is above the critical flicker frequency (CFF), the perceived durations are not dilated, suggesting that saliency is a critical factor for time dilation (Herbst et al., 2013). Other studies have demonstrated that neural entrainment is crucial for flicker-induced time dilation (Hashimoto \& Yotsumoto, 2015). In this study, we aimed to dissociate the effects of flicker saliency and induced neural oscillations by using intermodulation components that induce oscillations at the beat frequencies of two flicker frequencies above or below the CFF. Two stimuli, a standard and a comparison, were presented sequentially and the participants were asked to report which of the stimuli lasted longer. In Experiment 1, the standard stimulus was either below the CFF threshold (visible flicker), above the CFF threshold (invisible flicker), or both (intermodulation). The perceived duration was quantified and compared to that of the steady comparison stimulus. We found that the amount of dilation in the intermodulation condition was higher than that in the invisible condition, but lesser than that in the visible condition. In experiment 1 , however, the number of visible LED lights was not controlled across the conditions. Thus, it is possible that saliency affected the time dilation. Therefore, in experiment 2, we compared two combinations of the lights. One was a combination of an invisible flicker and a visible flicker, and the other was a combination of steady lights and a visible flicker. Although both the combinations induce the same perception, the combination including steady lights would not induce intermodulation. The amount of time dilation did not differ between the two combinations, indicating that at the tested frequency, saliency, not the intermodulation of neural entrainment, induced time dilation.

\title{
Keywords
}

Duration, behaviour, adult, 100s of ms-secs, flicker, time dilation

\section{References}

Hashimoto, Y., \& Yotsumoto, Y. (2015). Effect of temporal frequency spectra of flicker on time perception: Behavioral testing and simulations using a striatal beat frequency model. Timing \& Time Perception, 3, 201-222.

Herbst, S. K., Javadi, A. H., van der Meer, E., \& Busch, N. A. (2013). How long depends on how fast-perceived flicker dilates subjective duration. PLoS ONE, 8.

\section{Application of Fourier Analysis to Flicker Perception}

\author{
Anderson Rodrigues ${ }^{1}$, Eline Melo ${ }^{1,2}$ and Cláudio Teixeira ${ }^{1,2,3}$ \\ ${ }^{1}$ Núcleo de Medicina Tropical, Universidade Federal do Pará, Brazil \\ ${ }^{2}$ Centro de Ciências Biológicas e da Saúde, Universidade da Amazônia, Brazil \\ ${ }^{3}$ Centro Universitário do Estado do Pará, Brazil
}

The sensitivity to visual contrast of a luminous stimulus is determined by the amplitude of the fundamental Fourier component of the waveform at various spatial frequencies 
(Campbell \& Robson, 1968). On the other hand, interactions between the center and the periphery of neural fields determine the patterns of neural and perceptual response to the same set of luminous stimuli (Kremers et al., 2004; Teixeira et al., 2014). In this context, we test the hypothesis that such center- peripheral interactions are the neural mechanisms evidenced by Campbell and Robson. To this purpose, we quantified the perceived flicker strength (PFS) in the center of a test stimulus, which luminance was simultaneously modulated with a surround stimulus. The luminance modulation depth of a separate stimulus, identical to the center test stimulus but without the surround, was determined using a two- alternative forced choice procedure. The PFS was measured with no temporal phase differences between center and surround stimuli. Results show that: (i) there is a decrease in PFS when either sinusoidal center-peripheral stimuli or rectangular centerperipheral stimuli have the same contrast; (ii) there is a decrease in PFS, smaller than (i), when either a central sinusoidal stimulus and a rectangular peripheral stimulus or a central rectangular stimulus and a sinusoidal peripheral stimulus have the same contrast; (iii) there is a decrease in PFS, similar to (i) and larger than (ii), when either a central sinusoidal stimulus and a rectangular peripheral stimulus or a central rectangular stimulus and a peripheral sinusoidal stimulus have contrast levels differing by $4 / \pi$ times. In general, these results suggest that the strength of interactions between the center and the periphery of neural fields can be determined by the fundamental Fourier component of the waveform of the visual stimuli.

\title{
Keywords
}

Fourier analysis, lateral interaction, contrast sensitivity

\section{References}

Campbell, F. W., \& Robson, J. G. (1968). Application of Fourier analysis to the visibility of gratings. J. Physiol., 197, 551-566.

Kremers, J., Kozyrev, V., Silveira, L. C., \& Kilavik, B. E. (2004). Lateral interactions in the perception of flicker and in the physiology of the lateral geniculate nucleus. J. Vis., 4, 643-663.

Teixeira, C. E. C., Salomão, R. C., Rodrigues, A. R., Horn, F. K., Silveira, L. C. L., \& Kremers, J. (2014). Evidence for two types of lateral interactions in visual perception of temporal signals. J. Vis., 14, 1-18.

\section{Age-Related Effects on Center-Surround Interactions and Flicker Perception}

\author{
Cláudio Teixeira ${ }^{1,2,3}$, Beatrice Ono ${ }^{4}$, Anderson Rodrigues ${ }^{1}$ \\ and Jan Kremers ${ }^{5}$ \\ ${ }^{1}$ Núcleo de Medicina Tropical, Universidade Federal do Pará, Brazil \\ ${ }^{2}$ Centro de Ciências Biológicas e da Saúde, Universidade da Amazônia, Brazil \\ ${ }^{3}$ Centro Universitário do Estado do Pará, Brazil
}




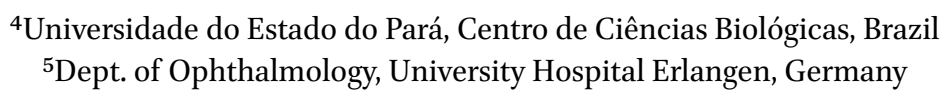

The aim of this work was evaluate the effects of aging on lateral interactions involved in flicker perception (Kremers et al., 2004; Teixeira et al., 2014) using psychophysical methods. To this purpose, we quantified the perceived flicker strength (PFS) in the center of a test stimulus, the luminance of which was simultaneously modulated with a surround stimulus eiter in phase or in counterphase. The luminance modulation depth of a separate (reference) stimulus, spatially identical to the center test stimulus but without the surround, was determined using a two-alternative forced choice procedure to achieve the same PFS as in the center of the test stimulus. The temporal frequency was $6 \mathrm{~Hz}$ in both reference and test stimuli. Using LCD goggles synchronized to the frame rate of a CRT screen, the center and the surround of the reference stimuli could be presented monoptically or dichoptically. Participants were healthy subjects with age between 27-60 years $(n=23)$. The results show that, when center and surround stimuli were modulated in counterphase and presented in monoptic condition, the PFS in the center stimulus decreased with age $(\mathrm{r}=-0.6478$; confidence interval $(95 \%)$ of $\mathrm{r}$ : -0.836 to -0.321 ; $\mathrm{p}=0.0008 ; \mathrm{R} 2=0,4196)$. In addition, we estimated the response amplitudes of a linear mechanism with center- surround receptive fields to the center $(\mathrm{rc}=(\mathrm{PFS1} 80+\mathrm{PFS0}) / 2)$ and the surround $(\mathrm{rs}=(\mathrm{PFS} 180-\mathrm{PFS} 0) / 2)$ stimuli using a linear model, where PFS0 is the psychophysical response to in-phase modulation and PFS180 is the psychophysical response to counter-phase modulation between center and surround stimuli. The evaluation of this linear mechanism showed that rc decreases with age when lateral interactions occur in monoptic test conditions $(r=-0.6848$; confidence interval $(95 \%)$ of $\mathrm{r}$ : -0.855 to $-0.379 ; \mathrm{p}=0.0003 ; \mathrm{R} 2=0.4689)$. The results indicate that monoptic lateral interactions mechanisms are more sensitive to aging.

\section{Keywords}

Aging, lateral interactions, contrast sensitivity

\section{References}

Kremers, J., Kozyrev, V., Silveira, L. C., \& Kilavik, B. E. (2004). Lateral interactions in the perception of flicker and in the physiology of the lateral geniculate nucleus. J. Vis., 4, 643-663.

Teixeira, C. E. C., Salomão, R. C., Rodrigues, A. R., Horn, F. K., Silveira, L. C. L., \& Kremers, J. (2014). Evidence for two types of lateral interactions in visual perception of temporal signals. J. Vis., 14, 1-18.

\section{Where Does Time Go When You Blink?}

\section{Shany Grossman ${ }^{1}$, Chen Gueta ${ }^{2}$, Rafael Malach ${ }^{\mathbf{1}}$ and Ayelet N. Landau ${ }^{2}$}

${ }^{1}$ Department of Neurobiology, Weizmann Institute of Science, Rehovot, Israel 
${ }^{2}$ Departments of Psychology and Cognitive Science, The Hebrew University of Jerusalem, Israel

Every time we blink retinal input is occluded for a considerable duration of $\sim 120 \mathrm{~ms}$. These frequent occlusions (every $\sim 5 \mathrm{~s}$ ) are barely noticed and perception remains seemingly continuous. Importantly, it has been shown that blinks are associated with a top down decrement in visual sensitivity (Volkmann et al., 1980). What are the consequences of such unnoticed occlusions on the subjective sense of time? Is the assessment of time altered by blinking? This ecological visual illusion offers an opportunity to study mechanisms of timing and how they relate to sensory input. We used a duration estimation task whilst monitoring participant's spontaneous blinks. Duration estimation was performed on a white circular stimulus subtending $6.67^{\circ}$ visual angle, presented at fixation. Participants were trained to discriminate between two anchor stimuli that defined either "short" or "long" intervals (600 or $2800 \mathrm{~ms}$, respectively). After mastering this discrimination, participants had to report whether the white circle, now presented at 13 intermediate intervals, was closer to the long or short anchor stimulus. This task allows the assessment of a "temporal bisection point", for which participants are as likely to judge the stimulus as "long" or "short". We found that at the temporal bisection point, participants were significantly more likely to judge the interval as "short" if a blink fell within that interval compared to a blink-free interval. Thus, the current findings suggest that the experience of time is compressed during spontaneous blinks. Does the effect point to a link between suppressed visual processing and time compression, or rather to a link between lapses in endogenous attention and time compression? Follow up experiments that will address the issue of domain specificity will further elucidate the origins of the current findings.

\title{
Keywords
}

Duration, behavior, adults, 100s of ms-secs

\section{Reference}

Volkmann, F. C., Riggs, L. A., \& Moore, R. K. (1980). Eyeblinks and visual suppression. Science, 207, 900-902.

\section{Time Dilation Is Independent of Stimulus Eccentricity in the Visual Field}

\author{
Xiaoxuan $\mathrm{Li}^{1}$ and Yan Bao ${ }^{1,2}$ \\ ${ }^{1}$ School of Psychological and Cognitive Sciences, Peking University, Beijing, China \\ ${ }^{2}$ Institute of Medical Psychology, Ludwig-Maximilians-University, Munich, Germany
}

It has been shown that cognitive processing is characterized by a functional dissociation in the visual field. Specifically, using inhibition of return (IOR) as an indicator it has been 
observed that attentional control underlies a functional subdivision showing different mechanisms in the periphery relative to the fovea and perifoveal regions of the visual field (Bao \& Pöppel, 2007; Yang et al., 2015). The present study aims to further address whether temporal processing of visual stimuli is also influenced by where the stimulus is presented. Previous study suggests that critical flicker fusion (CFF) is dependent on stimulus eccentricity reflecting presumably rather basic processes in the visual pathway. The question arises whether higher level temporal processing also shows such a dependency on visual field location. We addressed this question using time dilation effect as an indicator reflecting subjective duration estimation. With a duration judgment task a group of university students were asked to judge whether the systematically varied duration of a target (looming, receding or static visual disc) was longer or shorter than the duration of a standard stimulus (static disc). Time dilation effect (overestimation of the target duration) was observed for the looming target but not the receding target as compared to the standard duration of static stimulus. This effect was observed with no difference at both perifoveal and peripheral regions of the visual field. Although no difference cannot be used for logical reasons as argument that a difference does not exist, the result matches other observations that the stronger IOR effect in the periphery relative to the perifoveal IOR share a common time window of approximately three seconds. Thus, it can be concluded that time dilation effect is independent of stimulus eccentricity, suggesting a common temporal platform in the visual field.

\title{
References
}

Bao, Y., \& Pöppel, E. (2007). Two spatially separated attention systems in the visual field: Evidence from inhibition of return. Cogn. Process., 8, 37-44.

Yang, T., Zhang, J., \& Bao, Y. (2015). Spatial orienting around the fovea: Exogenous and endogenous cueing effects. Cogn. Process., 16S, 137-141.

\section{A Synchronising to a Frequency while Estimating Time, but just when You Are Aware of It}

\author{
D. A. Casilimas-Díaz and J. L. O. Bueno \\ Centre of Experimental Aesthetics, Department of Psychology, Faculty of Philosophy, \\ Sciences and Letters of Ribeirão Preto, University of São Paulo, Brazil
}

It is well known that subjective time perception can be modified by the emotional experiences related to a specific event, by pharmaceutical compounds, or by some sensory stimuli. From the latter, studies using visual (e.g., Nather et al., 2013) and auditory stimuli (e.g., Droit-Volet et al., 2010) prevailed compared with tactile ones (e.g., Hasuo et al., 2014). This study intended to fill this gap by assessing time perception differences between participants under different modalities of vibrotactile stimulation. To attend this purpose, two experiments were conducted using different vibratory frequencies to stimulate participants who were asked to reproduce stimulus' duration. Experiment 1 compared 
differences in reproduced time for stimuli between 0.5 and $6 \mathrm{~Hz}$, in 100 participants who performing a time reproduction task with the vibratory stimulus present or absent; also between groups performing the reproduction task using prospective or retrospective paradigm. Experiment 2 assessed differences in reproduced time by 80 participants under vibrotactile stimulation of two simultaneous frequencies delivered to each hand, those frequencies had specific proportions of 0.5 and 0.75 times the standard frequency for two groups of standard frequencies $(2$ or $12 \mathrm{~Hz}$ ). Reproduced times in Experiment 1 didn't show significant differences between frequencies, but did for absence/presence condition, solely, in prospective tasks, where estimations showed greater levels of overestimation in the absence of the vibrotactile stimulus while performing the time reproduction task. Significant differences were found in Experiment 2 for reproduced time by participants between two groups of standard frequencies. Data analysis suggest it is necessary to improve the understanding of subjective time perception processes for higher frequencies and considering intensity modulation based on the amplitude and frequency relation.

\title{
Keywords
}

Duration, behavior, adult, secs-mins, tactile system, reproduction task

\section{References}

Droit-Volet, S. (2010). Speeding up a master clock common to time, number and length? Behav. Processes, 85, 126-134.

Droit-Volet, S., Bigand, E., Ramos, D., \& Bueno, J. L. O. (2010). Time flies with music whatever its emotional valence. Acta Psychol., 135, 226-232.

Hasuo, E., Kuroda, T., \& Grondin, S. (2014). About the time-shrinking illusion in the tactile modality. Acta Psychol., 147, 122-126.

Nather, F. C., Mecca, F. F., \& Bueno, J. L. O. (2013). Motion illusions in optical art presented for long durations are temporally distorted. Perception, 42, 742-750.

\section{A Bayesian Perspective on Accumulation in the Magnitude System}

\author{
Benoit Martin', Martin Wiener ${ }^{2}$ and Virginie Van Wassenhove ${ }^{1}$ \\ ${ }^{1}$ Cognitive Neuroimaging Unit, CEA DSV/I2BM, INSERM, Université Paris-Sud, Université \\ Paris-Saclay, NeuroSpin center, Gif/Yvette, France \\ ${ }^{2}$ Department of Psychology, George Mason University, Fairfax, VA, USA
}

Several theoretical and empirical work posit the existence of a common magnitude system in the brain. Walsh's A Theory Of Magnitude (ATOM) proposes that analog quantities are mapped in a generalized magnitude system, which entails that space, time, and number may share a common neural code. Such a proposal implies that manipulating stimuli in one magnitude dimension (e.g., duration in time) should interfere with the subjective estimation of another magnitude dimension (e.g., size in space). Recent discussions in 
the field suggest that the combination and evaluation of quantities in a common representational system would be realized on the basis of Bayesian computations. Here, we asked whether a generalized Bayesian magnitude estimation system would sample sensory evidence using a common, amodal prior. In this study, participants were presented with clouds of dots, which appeared dynamically on a computer screen. A cloud of dots was characterized by its duration, its numerosity and its surface. Two psychophysical experiments separately tested participants on their perception of duration, surface, and numerosity when the non-target magnitude dimensions and the rate of sensory evidence accumulation were manipulated. First, we found that duration estimation was resilient to changes in surface and numerosity, whereas lengthening (shortening) the duration yielded under- (over-) estimations of surface and numerosity. Second, the perception of surface and numerosity were affected by changes in the rate of sensory evidence accumulation, whereas duration was not. Our results suggest that a generalized magnitude system based on Bayesian computations would minimally necessitate multiple priors. Duration estimates were not only resilient to changes in numerosity or surface, but also to the rate of sensory evidence. These findings suggest that unlike surface and numerical estimates, duration may not rely on the accumulation of discretized sensory evidence.

\title{
The Effect of Abstract Numerals on Long-Range Time Interval Estimation
}

\author{
Camila S. Agostino' ${ }^{1}$, Peter M. Claessens ${ }^{1}$, Fuat Balci ${ }^{2}$ and Yossi Zana ${ }^{1}$ \\ ${ }^{1}$ Federal University of ABC, Brazil \\ ${ }^{2}$ Koç University, Turkey
}

Most studies of time perception focused on the range of milliseconds, seconds and minutes. Consequently, there is limited knowledge of how people process long-range time intervals concerning from days to years. The aim of this study was to estimate the psychometric functions that describes long-range time interval perception and compare them with those of abstract numerals magnitude and future personal events. The working hypothesis was that the abstract numerals as part of the stimulus presentation influence the magnitude estimation of time intervals. The crossmodal line paradigm (Agostino et al., 2017; Zauberman et al., 2009) was used with three experimental groups. The participants of group I $(\mathrm{N}=20)$ estimated the magnitude of abstract numerals in the 3 to 36 range. The task for Group II $(\mathrm{N}=18)$ was to estimate the magnitude of time intervals indicated in the format "nn months". Participants of Group III ( $=19)$ estimated the magnitude of time intervals indicated by future personal events. Results showed that power functions provided a better fit to the data as compared to linear and logistic models, with BIC values at least 2 units lower. The estimates for the parameter of the power model were $0.84,0.77$, and 0.58 for Groups I, II and III, respectively. Nonlinear fixed-effects model analysis did not find significant difference between Group I and II ( $\mathrm{df}=413, \mathrm{t}=-1.09, \mathrm{p}>.27)$, but Group III was different from Group I $(\mathrm{df}=392, \mathrm{t}=-2.46, \mathrm{p}<.01)$ and Group II $(\mathrm{df}=414$, 
$\mathrm{t}=-3.72, \mathrm{p}<.005)$. This is the first study in which magnitude estimation of abstract numerals, long-range time interval and duration up to personal events are compared directly. The results suggest that participants ignore the time unit and consider primarily the magnitude of the attached numeral. It is concluded that experimental paradigms of measurement of long- range time perception should avoid the use of abstract numerals in stimuli presentation.

\title{
Keywords
}

Time perception, numerical estimation, personal events, power function

\section{References}

Agostino, C. S., Caetano, M. S., Balci, F., Claessens, P. M. E., \& Zana, Y. (2017). Individual differences in long-range time representation. Atten. Percept. Psychophys., 80, 1-8.

Zauberman, G., Kim, B. K., Malkoc, S. A., \& Bettman, J. R. (2009). Discounting time and time discounting: Subjective time perception and intertemporal preferences. J. Mark. Res., 46, 5 .

\section{The Impact of Perceptual/Concurrent and Mnemonic Digits on Temporal Processing: A Congruency Effect of Numerical Magnitudes}

\author{
Zhao Fan ${ }^{1,2}$, Guomin Jing ${ }^{1,2}$, Xianfeng Ding, ${ }^{1,2}$ and Xiaorong Cheng1,2 \\ ${ }^{1}$ Key Laboratory of Adolescent Cyber Psychology and Behavior (CCNU), Ministry of \\ Education, China \\ ${ }^{2}$ School of Psychology, Central China Normal University, China
}

Task-irrelevant stimulus numbers can automatically modulate concurrent temporal tasks-leading to the phenomenon of number-time association (NTA). Recent research provides converging evidence that the NTA occurs at the stage of temporal memory. Specifically, a reference memory containing encoded duration information can be modified by perceptual/concurrent digits, i.e., a perceptual/concurrent digit-induced NTA. Here, with five experiments, we investigated whether another working memory-related mechanism was involved in the generation of NTAs and how this memory-induced NTA was related with the perception-induced NTA. We first explored whether similar NTA effects existed for mnemonic digits, which disappeared before time encoding but were actively maintained in working memory, i.e., a mnemonic digit-induced NTA. Experiment 1 to 3 demonstrated both types of NTAs. Further, we revealed a close relationship between the two types of NTAs in two contexts. First, the mnemonic digit-induced NTA also relied on a perceptual number-time co-occurrence at time encoding. We found that the mnemonic digits influenced subsequent temporal processing when a task-irrelevant constant number ' 5 ' was presented during target encoding, but not when a non-numerical symbol was 
presented, suggesting that temporal representations in the reference memory could be accessed and modified by both sensory and postsensory numerical magnitudes through this number-time co-occurrence. Second, the effects of perceptual and mnemonic digits on temporal reproduction could cancel each other out. A congruency effect for perceptual and mnemonic digits (relying on memorization requirement) was demonstrated in Experiment $4 \& 5$. Specifically, a typical NTA was observed when the magnitudes of memorized and the perceptual/concurrent digits were congruent (both were large or small numbers), but not when they were incongruent (one small and one large numbers). Taken together, our study sheds new light on the mechanism of NTA.

\section{Keywords}

Duration, behavior, adult, memory number-time association

\section{Revisiting the Context Effect of Physical Quantity on Reproduced Durations}

\section{Stefan Pichelmann and Thomas Rammsayer}

University of Bern, Switzerland

In 2003, Walsh published his Theory of Magnitude (ATOM), which suggests a shared neural mechanism for the processing of time, space, and numerosity. Hence, estimations of time durations could be biased by concurrent magnitudes of nontemporal stimuli. Since then, several studies (e.g., Xuan et al., 2007) provided evidence for an influence of visually presented numerical values (Arabic digits) on estimated durations. Lu et al. (2009) suggested that the relationship between numerical digit value and perceived duration might be mediated/moderated by the kind of physical quantity participants associate with these numbers (e.g., kg vs mg) and reported some experimental support for their hypothesis. We conducted two experiments questioning this idea. Participants had to perform a time reproduction task with target durations ranging from 800 to $1200 \mathrm{~ms}$. Target intervals were presented visually using low $(1,2)$ or high $(8,9)$ numerical values combined with either a physical quantity with a small magnitude $(\mathrm{cm})$, a physical quantity with a high magnitude $(\mathrm{km})$, or no physical quantity. In both experiments, we showed a significant effect of numerical value on reproduced duration as presenting high numerical values during the target interval resulted in longer reproduced durations. Furthermore, our results suggest that physical quantity presented during the target interval cause longer reproduced durations when compared to time reproductions without providing a physical quantity during the target interval. Different magnitudes of physical quantity, however, did not show a significant influence on reproduced durations. There was, no evidence for a significant interaction between numerical value and physical quantity. In conclusion, we challenge Lu et al.'s notion of a context effect of physical quantities and rather argue for an effect of the area covered by the stimuli. 


\title{
Keywords
}

Duration, behavior, adult, 100s of ms-secs

\section{References}

Lu, A., Hodges, B., Zhang, J., \& Zhang, J. X. (2009). Contextual effects on number-time interaction. Cognition, 113, 117-122.

Walsh, V. (2003). A theory of magnitude: Common cortical metrics of time, space and quantity. Trends Cogn. Sci., 7, 483-488.

Xuan, B., Zhang, D., He, S., \& Chen, X. (2007). Larger stimuli are judged to last longer.J. Vis., $7,2-2$.

\section{Robustness of Individual Differences in Temporal Interference Effects}

\author{
Nadine Schlichting, Ritske de Jong and Hedderik van Rijn
}

Department of Experimental Psychology, University of Groningen, The Netherlands

The perceived duration of a specific event has been shown to be influenced by other dimensions of the very same event. In a previous experiment participants were presented with two stimuli consisting of small blue dots appearing and disappearing dynamically on the screen for different periods of time. Participant's task was to judge whether the second stimulus was shorter/longer (time condition) or consisted of fewer/more dots (numerosity condition) than the first stimulus. Using a Maximum Likelihood Estimation (MLE) procedure we estimated, for each participant separately, to what extent temporal and numerical information was taken into account in making judgments on either time or numerosity. Results showed that judgments on time were more likely to be affected by numerosity, while numerosity judgments were relatively resilient to temporal interferences. However, we found large individual differences in the magnitude of these interference effects (i.e., some participants relied almost exclusively on information of the relevant dimension, thus showing no or weaker interference effects). In the current study, we will test how stable these congruency effects are over time and over different task versions. Participants attended two sessions separated by six to eight days. During each session participants performed the dynamic magnitude task as described above, and a static version of the magnitude task (i.e., dots do not appear and disappear, but all dots are presented from onset to offset of the stimulus). In addition, participants performed a numerical Stroop task during Session 1, and a simple duration discrimination task during Session 2. This study-design enables direct comparison of MLE estimates over time (session one vs. session two) and between tasks (dynamic vs. static magnitude task). Further, we will assess whether magnitude of time- numerosity interference effects is related to effects in other interference and temporal judgment tasks (numerical Stroop and duration discrimination). 


\title{
Keywords
}

Magnitude processing, duration, behavior, adult, 100s of ms-secs

\section{The Mathematical Order Structure of Subjective Time}

\author{
B. Lungsi Sharma ${ }^{1}$ and Richard B. Wells ${ }^{2}$ \\ ${ }^{1}$ Centre National de la Recherche Scientifique. Unit of Neuroscience, \\ Information and Complexity, France \\ ${ }^{2}$ Emeritus Professor, University of Idaho, USA
}

As one interacts with the environment, the order of perception of objects and events are important. When you confront an object it's the knowledge that materializes. The knowledge that there is a pen, a paper and a desk in front of you is discovered instantly. Damasio (1999) hypothesizes that each materialized knowledge is the content of each pulse-like generation of consciousness. Studies suggests neural synchrony to play a role in temporal processing and consciousness (Meck et al., 2014). We may therefore look at temporal processing as that which engenders a sense of self in the act of knowing, an inner sense. The logical or mathematical view of the role of subjective time or time processing is hence the process of order structuring, the property that mathematicians refer to as weak and strict partial order; defined by reflexive, irreflexive, antisymmetric and transitive properties. Perception grows out of the previous moment and so on ... resulting in a timeline view of the chain. This is strict partial ordering. But this has the shortcoming that a moment in time is not relatable to itself. It has irreflexive property. A timeline view is therefore not sufficient to represent the time process. Partial ordering with the reflexive property is called weak partial ordering. This extends the one-dimensional timeline into a multi-dimensional timescape. The parts of the timescape represents markings of perceptions at moments in time. These are like Damasio's pulses of consciousness. The concept of multi-dimensional timescape is congruent with the massive parallelism of brain activity. The order structure is a system of self-regulatory transformations. A direct relationship of mental structures and mathematical structures was discovered by Piaget and Dieudonne (Piaget, 1970). This paper will present a mathematical description of "what time does for perception?".

\section{Keywords}

Synchrony, modeling, children, adult, 10s-100s of ms, mins-hours

\section{References}

Damasio, A. (1999). The feeling of what happens. New York: Harcourt, Brace \& Co.

Meck, W. H., Vatakis, A., \& van Rijn, H. (2014). Opening the door to theoretical discussions of consciousness, decision-making, multisensory processing, time cells and memory mapping ... to name but a few issues of relevance to temporal cognition. Timing \& Time Perception Reviews, 1, 1-4.

Piaget, J. (1970). Genetic epistemology. Translated by Eleanor Duckworth. New York: W.W. Norton \& Co. 


\title{
Distinct Roles for Supplementary Motor Area and Feature-Specific Regions of the Brain in the Subjective Distortion of Time
}

\author{
Pom Charras ${ }^{1}$ and Jennifer Coull ${ }^{2}$ \\ ${ }^{1}$ Département de Psychologie, Epsylon, EA 4556, Université Paul Valéry Montpellier 3, \\ Montpellier, France \\ ${ }^{2}$ Laboratoire des Neurosciences Cognitives UMR 7291, Aix-Marseille University, CNRS, \\ Marseille, France
}

In the last decade, a widespread cortico-striatal timing network has been described. A key role for the supplementary motor area (SMA) has been identified, with its activity varying as a function of stimulus duration. Although activity in feature-specific brain regions, such as occipital cortex, also increases with increasing duration, this occurs whether duration processing is task-relevant or not. By contrast, duration-related increases in SMA activity are evident only when participants are voluntarily timing stimulus duration(Coull et al., 2015). These results suggest that feature-specific activity in occipital cortex passively codes the elapse of time while activity in SMA reflects the active accumulation of temporal information. In the present fMRI study we aimed to identify feature-specific and feature-independent components of the timing network by using two distinct visual features to induce temporal distortions in perceived duration. Participants estimated brief durations (869-1739 ms) of visually presented stimuli that varied orthogonally with respect to two irrelevant dimensions: numerical magnitude (1/9) and flicker frequency (slow/fast). As expected, behavioral data analysis revealed that both dimensions led to temporal distortions (higher magnitude leading to longer perceived duration), though to a much larger extent for frequency than number. fMRI analyses identified a widespread timing network (SMA, right prefrontal cortex, and occipital areas) common to both conditions. Direct comparison of timing-related activity in frequency versus number conditions revealed feature-specific activity in ventral and dorsal regions of occipital cortex respectively. A more posterior region of occipital cortex was common to both conditions. Importantly, SMA activity was greater for large magnitude stimuli in the frequency versus number condition, consistent with behavioural evidence that frequency induced a greater degree of temporal distortion than number. These data indicate that interactions between keys nodes of the timing network and feature-specific regions of the brain may account for subjective distortions of time.

\section{Keywords}

Timing, context-dependent distortion, supplementary motor area, magnitude

\section{Reference}

Coull, J., Charras, P., Donadieu, M., Droit-Volet, S., \& Vidal, F. (2015). SMA selectively codes the active accumulation of temporal, not spatial, magnitude. J. Cogn. Neurosci., 27, 2281-2298. 


\title{
Spatial Task Difficulty Modulates Space-Time Interference
}

\author{
Kévin Vidaud-Laperriere and Pom Charras \\ Département de Psychologie, Epsylon, EA 4556, Université Paul Valéry Montpellier 3, \\ Montpellier, France
}

Space and time are intrinsically related in the human mind. Consequently, space affects time perception (referred to as the Kappa effect), and conversely (referred to as the Tau effect). But recent evidence for an asymmetrical advantage for space over time (Bottini \& Casasanto, 2013) called into question the reciprocal interference. Our objective was to investigate the strength of Kappa and Tau effects while manipulating spatial task difficulty. Based on recent evidence that space-time interference is modulated by spatial acuity (Cai \& Connell, 2015), we hypothesized that spatial judgments would be more influenced by irrelevant temporal information in a difficult than in an easy spatial task. Thirty-two participants performed both a temporal and spatial bisection tasks in two separate blocks. Within each trial, two dots were successively flashed at various locations, and participants had to judge, as either "short" or "long", the temporal or spatial interval between the two dots, depending on the experimental block. Spatial task difficulty varied across two experimental groups, whereas temporal task difficulty was kept constant. We analyzed the percentage of responses "long" (repeated-measure ANOVA). Our results clearly show that both spatial and temporal judgments are affected by irrelevant dimension (time and space respectively). More interestingly, the strength of the Tau and Kappa effects critically depend on spatial task difficulty. In the easy spatial task, the Kappa effect is much stronger than the Tau effect but the reverse pattern was observed in the difficult spatial task $[\mathrm{F}(1,30)=14.14, \mathrm{p}<.001]$. Our study provides evidence for flexible space-time interferences. When spatial task is highly demanding, participants are more influenced by irrelevant temporal information. The overall findings corroborate recent evidence for a role of visuospatial working memory in space-time interference (Starr \& Brannon, 2016) and support the interpretation of space-time interference in terms of gradient of automaticity.

\section{Keywords}

Magnitude, space-time interference, task difficulty, distance, duration

\section{References}

Cai, Z. G., \& Connell, L. (2015). Space-time interdependence: Evidence against asymmetric mapping between time and space. Cognition, 136, 268-281.

Casasanto, D., \& Boroditsky, L. (2008). Time in the mind: Using space to think about time. Cognition, 106, 579-593.

Starr, A., \& Brannon, E. M. (2016). Visuospatial working memory influences the interaction between space and time. Psychon. Bull. Rev., 23, 1839-1845. 


\title{
Attention from the Start to the End Point Forms Horizontal Mental Timeline
}

\author{
Daichi Yamashiro ${ }^{1}$ and Muneyoshi Hyodo ${ }^{2}$ \\ ${ }^{1}$ Graduate School of Letters, Chuo University, Japan \\ ${ }^{2}$ Faculty of Letters, Chuo University, Japan
}

Previous studies have shown that there is an association between space and time. For example, Santiago et al. (2010) reported that Spanish speakers, who have writing direction from the left to the right, showed that experienced events unfold along a left-to-right mental line. Furthermore, Ouellet et al. (2010) showed that the direction of time on the spatial representation coincides with the writing direction. In the present studies, we investigated whether the result of Santiago et al. could be replicated in Japanese speakers, who use vertical and horizontal writing directions on everyday life. In the first experiment, we asked participants to conduct temporal order comparison task, which was similar with Santiago et al.'s. In this task, participants learned a series of six image sequences, and then judged whether the images chosen randomly and presented had been presented before or later the reference point at the time of learning by the left and right key press. The result showed there was no association between space and temporal order statistically although the tendency showed the same as Santiago et al. The second experiment was conducted with the image sequences whose content connection eliminated by changing the combination of the image stimulus constituting the sequence. The result showed association between space and temporal order. When comparing the two experiments, it is indicated that participants in the former experiment were paying more attention around the reference point than both ends of the sequence, whereas participants in the latter experiment paid the same level of attention to the whole sequence during the order comparison task. These results suggest that it is necessary to pay attention to the elements from the beginning to the end in order to form the representation of temporal order on the horizontal space.

\section{Keywords}

Temporal order, space, mental timeline, reaction time

\section{Acknowledgements}

Tadeusz W. Kononowicz has been supported by ERC-YSt-263584 to Virginie van Wassenhove.

\section{References}

Ouellet, M., Santiago, J., Israeli, Z., \& Gabay, S. (2010). Is the future right time? Exp. Psychol., 57, 308-314.

Santiago, J., Román, A., Ouellet, M., Rodríguez, N., \& Pérez-Azor, P. (2010). In hindsight, life flows from left to right. Psychol. Res., 74, 59-70. 


\title{
Mental Time Lines: Dissociated Mechanisms from Different Ontogenetic Origins?
}

\author{
Xianfeng Ding, Zhongshu Li, Ning Feng, Xiaorong Cheng and Zhao Fan \\ School of Psychology, Central China Normal University (CCNU), Wuhan, P. R. China
}

\begin{abstract}
A growing body of evidence suggested that time can be represented separately on the horizontal, sagittal or vertical axis, in which the former one is originated from sensorimotor experiences and the latter two from spatial metaphors. However, little is known about how these mental time lines are activated. Are these mental time lines from different ontogenetic origins activated in the same way? The present study addressed this question using a multi-dimensional free-choice paradigm with Mandarin speakers in 4 experiments. The results showed that spatial-temporal congruency effects did not appear at the same time on sagittal and vertical axes, indicating no simultaneous activation for mental time lines from the same origin. On the contrary, spatial-temporal congruency effects were significant on the horizontal and the sagittal or vertical axes, indicating simultaneous activation for mental time lines from different origins. These findings supported that mental time lines with different ontogenetic origins have dissociated mechanisms, as they could be activated simultaneously with little or no competition between each other. Furthermore, it implied that low-level sensorimotor mechanism and high-level conceptual mechanism were concurrently involved in the process of time or other abstract concept.
\end{abstract}

Keywords

Order, language, behavior, adult, mins-hours

\section{An Overview of Instantaneous Modulations of Perceived Time}

\author{
Sarah Maass and Hedderik van Rijn \\ University of Groningen, The Netherlands
}

To everyone attending this first Conference of the Timing Research Forum, it will be obvious that interval timing is an important aspect of almost all laboratory tasks and everyday human behavior, ranging from giving a response before a deadline determined by the experimenter, to a speaker's selection of optimal pause durations to increase persuasiveness. However, it is essential to have reliable and validated methods to modulate subjective timing to test its influence on the performance on these "secondary tasks". With this poster, we will provide a visual overview of the types of modulations described in literature that have been shown to systematically affect the perception of time (in the millisecond to second range) without affecting the "secondary task" directly. This overview is based on an encyclopedic review of methods that have shown to modulate perceived 
time in single-session research studies conducted in laboratory settings. As such, we have explicitly excluded modulations of time induced by pharmacological manipulations, between-subject, repeated sessions, or cross-sectional or longitudinal studies (e.g., age or time-of-day effects). It is important to realize that different methods to affect subjective time will influence the participant in different ways. Therefore, depending on the "secondary task" that needs to be performed, some methods might be less suited to parameterize timing. The purpose of this overview is to aid researchers in selecting the most appropriate operationalization to study the influence of time on other cognitive tasks, both in laboratory settings and in more realistic, everyday contexts.

\title{
Multitasking and Ad Enjoyment: Does Our Perception of Time's Passage Mediate the Relationship between Multitasking and Ad Enjoyment
}

\author{
Shweta Balaji and Aoife McLoughlin \\ James Cook University, Singapore
}

Previous research on the effects of multitasking on media content have found that content can become more positively evaluated when one is doing multiple tasks (Voorveld, 2011). Multitasking may be particularly likely to affect advertising because ads are often avoided by engaging in another task during exposure to the ad content (Speck \& Elliott, 1997). When people multitask with media, ad messages are rarely the sole focus of attention. Chinchanachokchai et al. (2015) explored the relationship between multitasking and time perception as well as the relationship between multitasking and task enjoyment alongside ad evaluation. Their study found that the mediating role of the perception of time passing had a resulting positive effect on ad evaluation, and that positive evaluation was likely not due to limited capacity in the attention system. Rather, the feedback response to the ads appeared to be to the feeling that time seemed to pass unexpectedly fast. This paper presents work, which extends on Chinchanachokchai et al.'s project, again investigating the mediating effect of time perception on ad evaluation, this time using more generalizable, everyday tasks. Participants were test on one computer screen, which displayed between one and three tasks depending on condition. Tasks included Space Invaders gameplay, a reading comprehension task and television advertisement. Findings and implications are also discussed.

\section{Keywords}

Duration, behavior, adult, mins-hours

\section{References}

Chinchanachokchai, S., Duff, B. R., \& Sar, S. (2015). The effect of multitasking on time perception, enjoyment, and ad evaluation. Comput. Human. Behav., 45, 185-191. 
Speck, P. S., \& Elliott, M. T. (1997). Predictors of advertising avoidance in print and broadcast media. J. Advert., 26, 61-76.

Voorveld, H. A. (2011). Media multitasking and the effectiveness of combining online and radio advertising. Comput. Human. Behav., 27, 2200-2206.

\title{
Distorted Timing of One's Own Reaction Times in Multitasking Performance
}

\author{
Daniel Bratzke and Donna Bryce \\ Department of Psychology, University of Tübingen, Germany
}

In multitasking, two types of reaction time (RT) costs can be observed: dual-task costs and switch costs. Dual-task costs arise when people are confronted with more than one task at the same time, whereas switch costs arise when people must rapidly switch between different tasks. Throughout the past decade, several studies have demonstrated that the timing of one's own RTs in dual-task situations can be severely distorted. Introspective estimates of RTs (i.e., introspective RTs) usually do not reflect the objective dual-task costs suggesting that participants are largely unaware of their dual-task costs. These striking limitations of introspection about the temporal demands of one's own task processing have been attributed to a central processing bottleneck which blocks the conscious perception of taskrelevant stimuli. Alternative explanations emphasize the role of limited timing abilities in these highly demanding multitasking situations. In the present research, we extended the introspective RT approach to task switching paradigms. The results suggest that, in contrast to the previously observed unawareness of RT costs in dual-task situations, people are aware of their switch costs. Nevertheless, also in this multitasking situation timing of one's own RTs is not entirely free of distortions.

\section{Time Estimation Accuracy and Individual Characteristics}

\section{Katerina Sedlakova ${ }^{1,2}$, Adela Beckova ${ }^{1,3}$, Kristyna Maleninska ${ }^{1,4}$, Jan Rydlo', Jiri Horacek ${ }^{1,2}$ and Tereza Nekovarova ${ }^{1,2,5}$}

${ }^{1}$ National Institute of Mental Health, Klecany, Czech Republic

${ }^{2}$ Third Faculty of Medicine, Charles University in Prague, Prague, Czech Republic

${ }^{3}$ Department of Psychology, Faculty of Arts, Charles University, Prague, Czech Republic

${ }^{4}$ Department of Neurophysiology of Memory, Institute of Physiology, Academy of

Sciences of the Czech Republic v.v.i., Prague, Czech Republic

${ }^{5}$ Department of Zoology, Faculty of Natural Sciences, Charles University, Prague, Czech

Republic 
Time perception is a crucial cognitive domain. We focus on interval timing, as it is under the strongest cognitive and volitional control. Time estimation and its accuracy may be affected by various factors (e.g., type of task, personal characteristics or actual emotional state). In our study we focus on individual differences in time estimation accuracy and individual characteristics (as cognitive abilities or personality traits). We study time estimation accuracy in healthy volunteers who complete timing tasks, cognitive tests and personality questionnaires. To measure time estimation, we use repeated reproduction of rhythm tasks (the participant is asked to maintain the given frequencies with 300 or $900 \mathrm{~ms}$ inter-response intervals). Cognitive abilities (psychomotor speed, attention and working memory) are assessed by tests: Trail Making Test, Stroop test, Symbol-coding and Digit Span (WAIS-III). The PSDI (Personality Styles and Disorder Inventory) and NEO-FFI (NEO five-factor inventory) questionnaires are used to identify personality traits. Studying correlations between time estimation and other cognitive domains and personality traits may contribute to a deeper understanding of time perception mechanisms. Proposed test battery may be used not only in healthy volunteers but also in neuropsychiatric patients.

\section{Keywords}

Rhythms, neuropsychology, adult, 100s of ms-secs

\section{Acknowledgements}

This study was funded by the project Nr. LO1611 with a financial support from the MEYS under the NPU I program; by projects $260388 / S V V / 2017$ and Progres Q 35; and by grants no.15-29370A and 15-34524A.

\section{High Time Contraction in Young Children in Dual-Task Related to their Limited Attention Resources}

\section{Quentin Hallez and Sylvie Droit-Volet}

Université Clermont Auvergne, Laboratoire de psychologie sociale et cognitive, CNRS, UMR 6024, France

Numerous studies have shown that durations are judged shorter in a dual-task than in a simple-task condition. The resource-based theory of time perception suggests that this is due to the processing of temporal information, which is a demanding cognitive task that consumes limited attention resources. Our study investigated whether this time contraction in a dual-task condition is greater in younger children and, if so, whether this is specifically related to their limited attention capacities. Children aged from 5 to 7 years were given a temporal reproduction task in a simple and dual-task condition. In addition, 
different neuropsychological tests were used to assess not only their attention capacities but also their capacities in terms of working memory and information processing speed. The results showed a shortening of perceived time in the dual task compared to the simple task, and this increased as age decreased. The extent of this shortening effect was directly linked to younger children's limited attentional capacities: The lower their attentional capacities, the greater the time contraction was. This study demonstrated that children's errors in time judgments are linked to their cognitive capacities rather than to capacities that are specific to time.

\title{
Keywords
}

Children, duration, secs-min, attention, neuropsychology

\section{What's the Time? Clock Drawing Task in Patients with Brain Lesions}

\author{
R. Actis-Grosso' ${ }^{1}$, A. Lunardelli' ${ }^{2}$, G. Mioni ${ }^{3}$ and F. Stablum ${ }^{3}$ \\ ${ }^{1}$ Università degli Studi di Milano-Bicocca e Centro di Neuroscienze, Milano, Italy \\ ${ }^{2}$ Servizio di Neuropsicologia - Azienda Universitaria Integrata di Trieste, Italy \\ ${ }^{3}$ Dipartimento di Psicologia Generale, Università di Padova, Italy
}

The clock drawing test (CDT) is a widely used cognitive screening tool for the evaluation of cognitive decline. Here we present a tentative categorization of different errors as outcome of different pattern of cognitive deficits (e.g., difficulty in temporal orientation, working memory or difficulty in visuo-spatial representation). To this aim we collected and analyzed 50 neuropsychological protocols of patients with different cerebral lesions (i.e., traumatic brain lesions, stroke, etc.). The neuropsychological protocols included CDT and various measures of perception, visuo-spatial representation, attention and working memory. All these information were collected from the clinical records. Besides classical error classification by Rouleau et al. (1992) we identify other types of errors, mostly related with hands and numbers positions. We also identified some critical factors that may contribute to the different errors. In particular we identify as critical factors to be consider: 1) graphic difficulties; 2) conceptual deficit; 3) spatial and/or planning deficit; and 4) perseveration. Results will be discussed considering new implication for clinical practice and considering the different cerebral lesions of the patients.

\section{Reference}

Rouleau, I., Salmon, D. P., Butters, N., et al. (1992). Quantitative and qualitative analyses of clock drawings in Alzheimer's and Huntington's disease. Brain Cogn., 18, 70-87. 


\title{
Deteriorated Millisecond Timing and Deficient Executive Functions in Aging: Evidence on Common Neural Processes
}

\author{
Elzbieta Szelag1,2, Kamila Nowak ${ }^{1,2}$, Anna Dacewicz ${ }^{1}$, Anna Bombinska', \\ Katarzyna Broczek ${ }^{3}$, Malgorzata Kupisz-Urbanska ${ }^{3}$ \\ and Tadeusz Galkowski ${ }^{2}$ \\ ${ }^{1}$ Laboratory of Neuropsychology, Department of Neurophysiology, Nencki Institute of \\ Experimental Biology of Polish Academy of Sciences, Warsaw, Poland \\ ${ }^{2}$ University of Social Sciences and Humanities, Warsaw, Poland \\ ${ }^{3}$ Department of Geriatrics, Medical University of Warsaw, Warsaw, Poland
}

Aging produces declines in several cognitive processes, including executive functions (EF). The accumulated data have also indicated age-related deterioration in temporal information processing (TIP), specifically in millisecond timing (MT). The goal of the present study was to test the relationship between deteriorated MT and deteriorated EF in advanced age. Although in the previous studies both TIP and EF were investigated separately, to our knowledge no studies have examined the associations between age-related changes in these two domains. We tested 53 participants aged from 65 to 78 years, free of any neurological and psychiatric disorders, scored of at least 27 points on MMSE. Two procedures were applied: MT was assessed by sequencing abilities measured with auditory temporal-order threshold (ATOT). It was defined as the minimum time gap separating two auditory stimuli presented in rapid succession which is necessary for a subject to report their relation 'before-after' correctly. EF were assessed with the efficiency of the executive planning measured with the Tower of London-Drexel University Test (TOLDX), considering 7 outcome indices grouped into 3 main categories: Move Performance, Time Efficiency, and Violation Adherence. Using Spearman's rank correlations we observed two main results. Firstly, the indices of the TOLDX indicated a coherent construct, reflecting the effectiveness of executive planning in the elderly. Secondly, ATOT correlated significantly with the indices of TOLDX. Although some of these correlations were modified by the subject's age, the correlation between ATOT and the main index of TOLDX ('Total Move Score' from Move Performance category) was age resistant. These results suggest that normal aging may be characterized by an overlapping of deteriorated EF and deteriorated TIP. The relation 'EF-MT' may suggest common neural mechanisms underlying these two mental functions.

\section{Keywords}

Aging, temporal order, neuropsychology, 100s of ms, behavior, executive functions

\section{Acknowledgements}

Supported by NCN grant 2015/17/B/HS6/04182. 


\title{
Effects of the Manipulation of a Work of Art on Subjective Time in Patients with Parkinson's Disease
}

\author{
José Lino Oliveira Bueno ${ }^{1}$, Márcia Regina Motta ${ }^{1}$ and Vitor Tumas ${ }^{2}$ \\ ${ }^{1}$ University of São Paulo. Faculty of Philosophy, Sciences, and Letters of Ribeirão Preto, \\ Department of Psychology, Section of Psychobiology, Center for Experimental Aesthetics, \\ Ribeirão Preto, Brazil \\ ${ }^{2}$ University of São Paulo, Faculty of Medicine of Ribeirão Preto, Department of \\ Neuroscience and Behavioral Sciences, Section of Neurology, Ribeirão Preto, Brazil
}

Introduction: The subjective perception of time is fundamental to the conception of reality. Parkinson's disease (PD) is characterized by a dysfunction of dopaminergic system that is considered to be involved on temporal processing (Allman \& Meck, 2012). Experimental Aesthetics investigates the relation between art and behavior, thus it is possible to consider that the manipulation of an aesthetic object can affect temporal judgment (Berlyne, 1974). Aim: Examine how the manipulation of a work of mobile art affects subjective time in patients with PD. Methods: Ten participants diagnosed with PD and ten control participants manipulated two reproductions of artworks from the series "Bichos" by Lygia Clark with different complexity levels. After that, they performed a verbal estimation regarding the temporal duration of their manipulations. The procedure was recorded and then it was transcript and analyzed. The data were analyzed by Shapiro-Wilk, Mann-Whitney U and Wilcoxon tests. Results: All participants overestimated the manipulation time of the stimuli. However, subjects with PD showed less overestimation than control participants. In the PD group, manipulation time was shorter than the manipulation time in control participants. Four behavioral categories were analyzed: Touch, Move, Change of the artwork's placement and Release the piece of art (stimulus). The analysis of the exploratory activity of the participants showed that those with PD touched and released the stimulus more often and, in contrast, they moved and dislocated it less than the participants without the disease. Conclusion: The time estimated by the participants pointed out that the artwork manipulation altered the subjective perception of time. The explanation for these results can be related with the time manipulation of artworks, with consequent exploration of them and cognitive-motor processing of information.

\section{Keywords}

Time estimation, neuropsychology, Parkinson's disease, seconds, art, movement

\section{References}

Allman, M. J., \& Meck, W. H. (2012). Pathophysiological distortions in time perception and timed performance. Brain, 135, 656-677.

Berlyne, D. E. (1974). The new experimental aesthetics. In D. E. Berlyne (Ed.), Studies in the new experimental aesthetics (pp. 1-25). Toronto, Canada: University of Toronto. 


\title{
Timing as an Early Cognitive Signature of Alzheimer's Disease
}

\section{Chasity Chung and Charlotte Bonardi}

The University of Nottingham, UK

\begin{abstract}
Alzheimer's Disease (AD) is considered the most common form of dementia. Currently, diagnosis remains restricted to the onset of memory problems, by which point the disease has progressed significantly (Sperling et al., 2011). Therefore, it is paramount to uncover methods to diagnose AD preclinically, before memory deficits manifest. Previous study with both humans and animals (Bonardi \& Armstrong, 2015; Rueda \& SchmitterEdgecombe, 2009) have found that deficits in timing precision are present in early and mild AD. However, this has not been explored in depth as a potential cognitive signature for AD, particularly for those who have been diagnosed with Mild Cognitive Impairment, often considered a preliminary diagnosis or 'stable form' of dementia. We have examined whether timing ability can be used to differentiate AD from other forms of dementia. Our current study tests this possibility with 3 clinical populations: those with $\mathrm{AD}$, those with other types of dementia, and those with MCI, as well as a healthy aging control group. Participants are given a computer task and are told to produce a target time of one of five given intervals (e.g., 1.3s) by pressing the space bar. Each interval is repeated twelve times; on half the trials participants are given feedback on their exact reaction time, but in the remaining trials, participants receive no feedback. The results are then sorted into $0.1 \mathrm{~s}$ bins and fitted to Gaussian curves, to establish timing precision and accuracy in the various conditions. The extent to which these timing parameters can discriminate between the different groups of participants will be evaluated.
\end{abstract}

\section{Keywords}

Duration, neuropsychology, aging, Alzheimer's Disease, dementia, 100s of ms-secs

\section{References}

Bonardi, C., \& Armstrong, P. (2016). Deficits in object-in-place but not relative recency performance in the APPswe/PS1dE9 mouse model of Alzheimer's disease: Implications for object recognition. Behav. Brain Res.

Rueda, A. D., \& Schmitter-Edgecombe, M. (2009). Time estimation abilities in mild cognitive impairment and Alzheimer's disease. Neuropsychology, 23, 178-188.

Sperling, R. A., et al. (2011). Toward defining the preclinical stages of Alzheimer's disease: recommendations from the National Institute on Aging-Alzheimer's Association workgroups on diagnostic guidelines for Alzheimer's disease. Alzheimers Dement, 7 , 280-292. 


\title{
Psychological Time in MCI Patients
}

\author{
Sara Coelho ${ }^{1}$, Manuela Guerreiro, Catarina Chester, Dina Silva, \\ Joao Maroco, Miguel Coelho, Fabio Paglieri \\ and Alexandre De Mendonça \\ ${ }^{1}$ CEA, DSV/I2BM, NeuroSpin; INSERM, U992, Cognitive Neuroimaging Unit; \\ Univ Paris-Sud, F-91191 Gif/Yvette, France
}

\begin{abstract}
During consultation, mild cognitive impairment (MCI) patients often referred difficulties in dealing with time questions, an issue which has consequences in important life domains such as decision-making and planning. Psychological time may be approached through the concepts of duration, succession and time perspective. Duration concerns how long an event takes, comprising estimation abilities and subjective passage of time judgments. Succession means temporal order. Time perspective concerns our views about the past, the present and the future. On the other hand, MCI patients can present us a human disease model to see if and how memory impairments affect human psychological time. Our study proposes to evaluate the psychological time in MCI patients, concerning the aspects of duration and time perspective. We will focus on the perception of short intervals' duration and also test the perceive speed of time passage for long intervals. Together, these two experiments will show us how MCI may differ in time duration from healthy aged-controls. Additionally, we want to see how MCI patients value each time dimension trough a time orientation-perspective task. Methods: Fifty-five MCI patients and fifty-seven healthy controls were submitted to a neuropsychological evaluation, an experimental protocol for time duration on short intervals and a questionnaire for time awareness. The survey concerning time orientation-perspective task is still ongoing. Patients with MCI exhibited no alterations in the perception of short intervals. However, results showed that MCI patients felt the time passing slower than controls and this result was significantly correlated with memory deficits. Memory deficits affect the experience of the perceived speed of time course but not the perception of the interval length.
\end{abstract}

Keywords

Duration, mild cognitive impairment, seconds/minutes, time perspective

\section{Does Time Speed Up when Thoughts Race? The Experience of Time in Adult Attention Deficit/ Hyperactivity Disorder and Bipolar Disorder}

\author{
Luisa Weiner, ${ }^{1,2}$, Anne Giersch ${ }^{1,2}$, Gilles Bertschy ${ }^{1,2}$ \\ and Sébastien Weibel',2 \\ ${ }^{1}$ Hôpitaux Universitaires de Strasbourg, France \\ 2INSERM U1114, France
}


Overestimation of durations has been reported in individuals with Attention-Deficit Hyperactivity Disorder (ADHD), but also in bipolar disorder (BD). Inattention and racing thoughts are symptoms shared between the two disorders, and might be associated with distortions in duration estimation and in the feeling of the passage of time. However, the relation between the speed of thoughts, attention, and working memory impairment, on the one hand, and duration perception, on the other hand, has been underexplored in both groups of patients. We compared performance of 18 euthymic patients with BD, 17 unmedicated adults with ADHD, and 27 controls on verbal estimation and production tasks in the multiple seconds range, and subjective perception of the passage of time. Additionally, participants filled out a self-report measure of racing thoughts, and several neuropsychological tests. The ADHD group significantly overestimated durations compared to the $\mathrm{BD}$ group, although both groups of patients showed significant attention and working memory difficulties. Thus, overestimation of durations in ADHD seem independent from working memory and attention dysfunction. The three groups did not differ in their judgment of the passage of time. However, the ADHD group showed elevated racing thoughts relative to the other groups, and these scores were correlated with the judgment of passage of time. In the ADHD group, a relative slowing of the passage of time was associated with self-reported accelerated thoughts, whereas the reverse pattern was observed in healthy controls. Hence the ADHD group seem to rely on duration estimates, and the amount of internal events, when emitting judgments on the passage of time: the more their thoughts are racing, the longer/slower their judgment of the passage of time. In contrast, the results in controls might be seen as consistent with recent proposals that increased alertness is associated with the relative feeling that time passes fast.

\title{
Keywords
}

Duration, behavior, adult ADHD, bipolar disorder, secs-mins

\section{Time Perception and Autistic Spectrum Condition: A Systematic Review}

\author{
M. Casassus ${ }^{1,2}$, E. Poliakoff ${ }^{1}$, D. Poole ${ }^{1}$, E. Gowen ${ }^{1}$ and L. A. Jones ${ }^{1}$ \\ ${ }^{1}$ University of Manchester, Manchester, UK \\ ${ }^{2}$ Head Forward Centre, Manchester, UK
}

Problems with timing and time perception have been suggested as key characteristics of Autism Spectrum Condition (ASC). Some studies and personal accounts from clinicians, parents, caregivers and self-reports from autistic people themselves often refer to problems with time, whilst other studies have found no impairment or differences between ASC and the general population. The results and findings from previous empirical research do not point into to a definitive conclusion, a key reason for this is because of the wide range of methodologies, research paradigms and samples they have used (Jones et al., 2017). In order to gain more clarity regarding what the current scientific view and 
weight of evidence is on this issue, a systematic review was conducted. Five electronic databases were consulted. From initial 597 records (after duplicates were removes), 47 papers were selected and reviewed. Different sections are presented for time sensitivity, interval timing and high-order time perception research. Within each section cognitive models, methodologies, possible clinical implications and research results are discussed. Our results showed no clear conclusion about whether ASC people have pure time perception impairment. However, some trends across studies show that studies in children are more likely to find impairment in ASC than studies in adults. A possible developmental component and a future plan of research are discussed.

\section{Keywords}

Duration, behaviour, Autism, systematic review

\section{Reference}

Jones, C. R. G., et al. (2017). Using time perception to explore implicit sensitivity to emotional stimuli in Autism Spectrum Disorder.J. Autism. Dev. Disord., 1-13.

\section{Time-Based Event Expectancies in Children with Autism Spectrum Disorders}

\section{Marina Kunchulia', Tamar Tatishvili ${ }^{1}$, Khatuna Parkosadze ${ }^{1}$, Nino Lomidze ${ }^{\mathbf{1}}$ and Roland Thomaschke ${ }^{2}$}

${ }^{1}$ Institute of Cognitive Neurosciences, Agricultural University of Georgia, Tbilisi, Georgia

${ }^{2}$ Department of Psychology, University of Freiburg, Freiburg, Germany

Research has shown abnormal development of temporal cognition in individuals with Autism Spectrum Disorders (Falter \& Noreika, 2011; Szelag et al., 2004). However, it is currently unclear how Autism Spectrum Disorders effect on time-based event expectancies. The aim of the study was to investigate the development of time-based event expectancies in children with Autism Spectrum Disorders. Ten children with Autism Spectrum Disorders and ten age-matched (6-11 years) typically developing children participated. Children with Autism Spectrum Disorders had no signs of the mental retardation as tested with non-verbal IQ test (TONI-4). In a choice-response task with two different pre-target intervals, participants had to indicate the left or right direction of a target stimulus. The target was predicted by the duration of the pre-target interval with $80 \%$ validity. We found that in children with Autism Spectrum Disorders, in contrast to typically developing children, the formation of the time-based event expectancies was restricted to the relatively longer pre-target interval. This pattern is rather typical for healthy young adults. These findings indicate that the children with Autism Spectrum Disorders can form time-based event expectancies, and that, similar to healthy young adults, the longer pre-target intervals are more optimal to make temporal predictions. 


\title{
Keywords
}

Prediction, neuropsychology, Autism Spectrum Disorders, 100s of ms-secs, time-based expectation

\section{References}

Falter, C. M., \& Noreika, V. (2011). Interval timing deficits and abnormal cognitive development. Front. Integr. Neurosci., 13, 5-26.

Szelag, E., Kowalska, J., Galkowski, T., \& Pöppel, E. (2004). Temporal processing deficits in high functioning children with autism. Br. J. Psychol., 95, 269-282.

\section{The Psychopathology of Time and Timing as Seen in Major Depressive Disorder and Autism-Spectrum Disorder}

\author{
David Vogel and Kai Vogeley \\ Department of Psychiatry, University Hospital Cologne, Germany
}

Time influences and shapes our perception of self. Recognizing time as both a perception and an experience is a key constituent to understanding our subjective experience. The experience of time and of its passage allows us to refer to different temporal dimensions, namely past, present, and future. It enables us to integrate previous life experiences and to prepare goal-directed actions. Without an inner experience of time we could neither experience nor plan our behavior, our actions or their results. Although the experience of time is of central relevance for psychopathology, qualitative approaches to study the inner experience of time have been largely neglected. We present the results form qualitative data based on summarizing content analysis obtained from 25 subjects with Major Depressive Disorder (MDD) and/or a history of MDD, 25 subjects with autism-spectrum-disorder (ASD), and 25 healthy control persons. All diagnostic groups show distinct differences in their experience of time. Healthy controls exhibit a sense of directedness through planning and striving towards individual goals and thereby seizing provided opportunities. In case of the ASD group, the approach to goal-directed action is defined by an isolated present with specific conduct, which strongly dominates future planning to reduce futureassociated ambiguities and insecurities. In case of the MDD group, planning and execution capabilities are diminished, thus limiting the ability to influence or change the individual present, thereby blocking the personal future and turning time into an inexorable continuance. The qualitative approach potentially allows researchers to develop new and innovative strategies to promote experimental studies and to explore the subjective experience of persons with mental disorders with the aims of an improved differential diagnosis and potentially new therapeutic strategies for the mental disorders studied.

\section{Keywords}

Duration, neuropsychology, adult, depression, autism, mins-hours 


\title{
Time Perception, Symptoms and Emotions in Psychiatric Conditions: A Daily Life Study
}

\author{
Maud Dupuy ${ }^{1,2}$, Anthony Roy', David Misdrahi ${ }^{1,3}$, Bernard N'Kaoua ${ }^{2}$, \\ Arnaud Tessier ${ }^{1,3}$, Alexandra Bouvard ${ }^{1}$, Pierre Schweitzer ${ }^{1,4}$, \\ Marc Auriacombe $^{5}$, Fuschia Serre ${ }^{5}$, Melina Fatseas ${ }^{5}$, Joel Swendsen ${ }^{1,4}$ \\ and Micha Pfeuty ${ }^{1}$ \\ ${ }^{1}$ University of Bordeaux, CNRS UMR 5287, Bordeaux, France \\ ${ }^{2}$ University of Bordeaux, EA 4136 - Handicap Activité, Cognition, Santé, Bordeaux, \\ France \\ ${ }^{3}$ Pole de Psychiatrie 347, C.H. Charles Perrens, Bordeaux cedex, France \\ ${ }^{4}$ PSL Research University, EPHE, Bordeaux, France \\ ${ }^{5}$ University of Bordeaux, PSL Research University, Addiction psychiatry Team, SANPsy \\ CNRS USR 3413, Pôle Addictologie, CH Charles Perrens and CHU de Bordeaux, \\ Bordeaux, France
}

Alterations of time perception in psychiatric disorders are a well-known phenomenon, both in regards to time interval estimation and to the subjective experience of time flow. A dysfunction in time perception may be involved in the daily living difficulties experienced by these patients. However, thus far, studies exploring deficits in time estimation among persons with psychiatric conditions were conducted in laboratory conditions, making it impossible to observe dynamic fluctuations of time perception as they relate to the expression of symptoms and emotions. This investigation examines the unexplored relationship between time perception and variability of symptoms and emotion in persons with schizophrenia, depression and substance dependence as compared to healthy individuals. To assess this, we used Ecological Momentary Assessment (EMA) that consists of smartphones apps delivering 5 interviews per day for one week, allowing the collection of extensive information concerning experience of time flow, verbal time estimation, symptoms and emotions in real-time and in natural settings. To date, 28 individuals with schizophrenia, 16 individuals with depression, 37 individuals with substance dependence and 27 healthy controls participated in the study. Preliminary analyses reveal that fluctuations of time estimation with emotional states differ significantly between groups. Among them, controls overestimated time intervals when they express positive emotional states, whereas patients with psychopathology underestimated time intervals when experiencing these same emotions. Interestingly, patients experienced time flow as more variable compared to controls and their experience of the passage of time was related to the nature of symptoms, such as in schizophrenia where negative symptoms were associated with slower and less variable experience of time flow and positive symptoms were associated with more variable time-flow experience. Finally, the information collected through EMA was correlated with data from morphological and functional neuroimaging techniques, which should enable the characterization of specific neurobiological markers of temporal processing in psychiatric conditions. 


\title{
Keywords
}

Duration, MRI, schizophrenia, depression, addiction, secs-mins

\section{Time Perception and Temporal Processing in Schizophrenia: A Meta-Analysis}

\author{
Sven Thoenes ${ }^{1}$ and Daniel Oberfeld ${ }^{2}$ \\ ${ }^{1}$ Leibniz Research Centre for Working Environment and Human Factors, Dortmund, \\ Germany \\ ${ }^{2}$ Experimental Psychology, Department of Psychology, Johannes Gutenberg-Universität \\ Mainz, Germany
}

Research on time perception and temporal processing in clinical populations indicates temporal impairments in patients with schizophrenia. However, past studies largely differ in the tasks and methods used, and the outcomes of the empirical studies do not always agree. In a meta-analytical review (Thönes \& Oberfeld, 2017), we differentiate between time perception (judgments of duration) and basic temporal processing (judgments of temporal order and simultaneity), as well as between effects on accuracy (deviation of mean judgments between patients and control participants) and precision (variability of judgments). In a meta- regression approach, we also included the specific temporal tasks and the different time interval ranges as covariates. We considered 68 publications of the past 65 years, and meta- analyzed data from 957 patients with schizophrenia and 1060 healthy control participants. Independent of tasks and interval durations, our results demonstrate that time perception and basic temporal processing are less precise (more variable) in patients (Hedges' $g>1.00$ ). Effects of schizophrenia on accuracy of time perception are rather small and task-dependent, but cannot be attributed to specific memory or motor demands. Our review also shows that several aspects, e.g., potential influences of medication, have not been investigated in sufficient detail yet. In conclusion, the results are in accordance with the notion of a more variable internal clock in patients with schizophrenia, but not with a strong effect of schizophrenia on clock speed. The results are also compatible with the notion of mistimed information transfer in schizophrenia by Andreasen et al. (1999), who have provided a popular framework for the relationships between basic cognitive impairments and the clinical outcome in schizophrenia. Future studies should try to combine experimental and phenomenological approaches in order to gain a broader understanding of the temporal distortions in schizophrenia and, more general, of the disease as such.

\section{Keywords}

Duration, order, behavior, neuropsychology, schizophrenia, all time scales 


\title{
References
}

Andreasen, N. C., Nopoulos, P., O’Leary, D. S., Miller, D. D., Wassink, T., \& Flaum, L. (1999). Defining the phenotype of schizophrenia: Cognitive dysmetria and its neural mechanisms. Biol. Psychiatry, 46, 908-920.

Thönes, S., \& Oberfeld, D. (2017). Meta-analysis of time perceptionand temporal processing in schizophrenia: Differential effects on precisionandaccuracy. Clin. Psychol. Rev., 54, 44-64.

\section{Borderline or Bipolar? Differences in Time Perception}

\section{Shima Talehy-Moineddin ${ }^{1}$, Arash Zandparsa ${ }^{2}$ and Homa Mohammadsadeghi ${ }^{2,3}$}

\author{
${ }^{1}$ School of Cognitive Sciences, Institute for Research in Fundamental Sciences, \\ Tehran, Iran \\ ${ }^{2}$ Psychiatry Departments, School of Medicine, Iran University of Medical Sciences, \\ Tehran, Iran \\ ${ }^{3}$ Mental Health Research Center (MHRC), Tehran, Iran
}

We investigated the time estimation in two groups of patients with borderline personality disorder (BPD) and bipolar disorder (BD), and compared it with the healthy controls (HC). We hypothesized that the time estimation in groups of BPD and BD is different from each other and also from HC. Patients were diagnosed based on DSM IV criteria and structured clinical interviews (SCID-I and SCID-II). Each time interval was presented randomly 6 times in two separate blocks of visual or auditory stimulus. In the visual block, a yellow circle was presented on the computer screen while in the auditory block a pure tone was continuously played through the speaker. The participant was required to judge the duration of stimulus presentation at the end of the trial. To prevent counting as a timing strategy, a working memory task accompanied trials. A three-digit number was presented to the participant at the start of each trial and needed to be recalled at the end of the trial. We fitted a line to the perceptual time (tp) of subjects in each group as a function of the stimulus presentation time (ts) with $t p=a^{*} t s+b$. In the BD group, the slope of the fit was 0.61 with an intercept of 6.46 while the slopes for BPD and HC groups were 1.00 and 0.85 , respectively. In addition, we performed ANOVA to investigate the significant group differences for time intervals. The three groups were different at four intervals of $5 \mathrm{~s}$ $(p=0.04), 18 \mathrm{~s}(\mathrm{p}<0.01), 34 \mathrm{~s}(\mathrm{p}=0.03)$ and 65s $(\mathrm{p}<0.01)$. In conclusion, the average estimated-time reported by BPD group was longer than that of the HC group while BP's time estimation was on average shorter than both of BPD and HC.

\section{Keywords}

Duration, behavior, adult, borderline personality disorder, bipolar disorder, secs-mins 


\title{
Depression Can Cause to Time Compression during Emotional Oddball Stimuli
}

\author{
Mahsa Ghaheri ${ }^{1}$ and Amir Hossein Ghaderi ${ }^{2}$ \\ ${ }^{1}$ Department of psychology, Najafabad Branch, Islamic Azad University, Najafabad, Iran \\ ${ }^{2}$ Cognitive Neuroscience Lab., Department of psychology, University of Tabriz, \\ Tabriz, Iran
}

Persons with major depression disorder (MDD) report underestimation of time (Gil \& Droit-Volet, 2009). The novelty of the stimuli may cause to subjective time dilation. Repeated stimuli are perceived shorter than novel stimuli and this effect may be depended to repetition suppression (Pariyadath \& Eagleman, 2012). However, there is no any investigation in people with major depression disorder. According to underestimation of time among persons with MDD, it seems different mechanism in time perception of oddball/ repeated may involve. In this study, oddball paradigm was used for comparison of time discrimination between subjects with major depression disorder assessed for with the Beck depression Inventory ( $\mathrm{N}=15,20$ and 35 years old; SD: 3:12) and normal control subjects ( $\mathrm{N}=15,20$ and 35 years old; SD: 2.89). Faces with neutral, happy and sad expressions were used as frequent and rare stimuli. Durations of stimuli were under 1 second and task was in accordance with previous study accomplished by Pariyadath and Eagleman (2012). The results showed time discrimination between two groups was significantly different and the MDD group show more error in time discrimination in the face of sad-neutral and happy- neutral stimuli. Despite the normal group, oddball stimuli were underestimated by the MDD group. The results suggest that mechanism of time discrimination of emotional stimuli in MDD is altered. It may be dependent to reduced activity of striatum (as clock) in the face of emotional stimuli (Epstein et al., 2006). Reduced activity of striatum may reduce speed of internal clock and cause to underestimation of emotional oddball stimuli. This result is in contradiction with time compression during repetition suppression and may suggest that time dilation during oddball stimuli were not established for persons with MDD. According to results, time perception in the MDD group may explain within internal clock model.

\section{Keywords}

Duration, depression, 100s of ms-secs, emotion, repetition suppression, internal clock

\section{References}

Gil, S., \& Droit-Volet, S. (2009). Time perception, depression and sadness. Behav. Processes, 80, 169-176.

Epstein, J., Pan, H., Kocsis, J. H., Yang, Y., Butler, T., Chusid, J., Hochberg, H., Murrough, J., Strohmayer, E., Stern, E., Silbersweig, D. A. (2006). Lack of ventral striatal response to positive stimuli in depressed versus normal subjects. Am.J. Psychiatry, 163, 1784-1790.

Pariyadath, V., \& Eagleman, D. M. (2012). Subjective duration distortions mirror neural repetition suppression. PLoS One, 7, p.e49362. 


\title{
Can Training Induce Changes in Temporal Dynamics of Brain Processes in Two Different Time Integration Windows in Children with Specific Language Impairment?
}

\author{
Anna Dacewicz ${ }^{1}$, Aneta Szymaszek ${ }^{1,2}$, Kamila Nowak ${ }^{1,2}$, \\ Anna Bombinska ${ }^{1}$ and Elzbieta Szelag1,2 \\ ${ }^{1}$ Laboratory of Neuropsychology, Department of Neurophysiology, Nencki Institute of \\ Experimental Biology of Polish Academy of Sciences, Warsaw, Poland \\ ${ }^{2}$ University of Social Sciences and Humanities, Warsaw, Poland
}

Children with Specific Language Impairment (SLI) are disordered in language acquisition however, their general cognitive functioning remains in normal range. The etiology of the SLI remains unclear. Therefore, conventional speech therapy is not always efficient. Our previous studies (Szelag et al., 2015) revealed that audiovisual computer training in temporal information processing (TIP) triggered improvement in both language and cognitive functioning. The main aim of the present study was to investigate the effects of such training in perspective of changes in brain processes. Thirty-six children between 5-8 years (26 boys) diagnosed with SLI underwent two types of intense intervention i.e., experimental temporal training $(\mathrm{n}=18)$ and control non-temporal training $(\mathrm{n}=18)$. Before and after the training auditory event related potentials (ERP) were measured in an oddball paradigm. We examined the brain responses to tones pairs of 1000 and $1200 \mathrm{~Hz}$ presented with Inter-Stimulus Intervals (ISIs) of 50 and $200 \mathrm{~ms}$. These ISIs have reflected the length of the two time integration windows involved in different levels of speech processing. The short window $(50 \mathrm{~ms})$ is crucial for phoneme reception, whereas, the long one $(200 \mathrm{~ms})$ contributes to syllable processing. After 24 training sessions both types of training induced enhancement in P3a amplitude, irrespective of the ISI condition, which may be due to attentional compensatory mechanisms. But only after the temporal training greater mismatch negativity (MMN) amplitude was observed in both ISIs conditions, reflecting increased efficiency of perceived change in rapid auditory sequences. Enhanced brain processes related to temporal binding within both 50 and $200 \mathrm{~ms}$ ranges may constitute the neural underpinning of language and cognitive improvement following the training.

\section{Keywords}

Specific language impairment, event related potentials, temporal processing, sensory, temporal binding window, cognitive training

\section{Reference}

Szelag, E., Dacewicz, A., Szymaszek, A., Wolak, T., Senderski, A., Domitrz, I., \& Oron, A. (2015). The application of timing in therapy of children and adults with language disorders. Front. Psychol., 6. 


\title{
The Role of Vision in Time Perception: Evidence from Sighted and Severely Visually Impaired Children
}

\author{
Tiziana Battistin ${ }^{1}$, Vittorina Schoch ${ }^{1}$, Giovanna Mioni ${ }^{2}$ \\ and Patrizia Bisiacchi ${ }^{2}$ \\ ${ }^{1}$ Robert Hollman Foundation, Padova, Italy \\ ${ }^{2}$ Department of General Psychology, University of Padova, Italy
}

In literature, some authors supposed the existence of a system with more "internal clocks", depending on the sensory modality used to present temporal stimuli. The aim of the present study is to investigate time perception in severely visually impaired children and sighted children. In Experiment 1, eighteen severely visually impaired and twenty sighted children, ranging in age from 6 to 11 years, performed a computerized time bisection task, which consisted in judging different temporal intervals using an acoustic stimuli. In the learning phase, children listened ten times a brief sound (300 ms) and ten times a long sound $(900 \mathrm{~ms})$. In the experimental phase, test stimuli $(300,400,500$, 600, 700, 800 and $900 \mathrm{~ms}$ ) were presented randomly in four blocks of sounds, with 42 stimuli/block. Children had to evaluate if the perceived stimuli were more similar to the brief or to the long sound, by pressing a specific tactile button on the keyboard. Results on experiment 1 showed the possibility either of network reorganization due to plasticity in visually impaired children or of the presence of an interference with the visual modality in sighted ones. Experiment 2 reproduced the same bisection task in a group of 18 sighted children, who were blindfolded, to exclude the visual modality, in order to check if the result was the same or not. The obtained data are interpreted in the light of inter-modality interference, assuming that, in controls, the possibility of using either sensory modalities could create an interference, which burdens on a major allocation of attentional resources.

\section{Keywords}

Time perception, interval timing, temporal accumulation, temporal decision, decision making, contingent negative variation, N1P2, LPCt

\section{References}

Bueti, D. (2011) The sensory representation of time. Front. Integr. Neurosci., 5, 34.

Droit-Volet, S. (2013) Time perception in children: A neurodevelopmental approach. $\mathrm{Neu}$ ropsychologia, 51, 220-234.

Grondin, S. (2010) Timing and time perception: A review of recent behavioural and neuroscience findings and theoretical directions. Atten. Percept. Psychophys., 72, 561-582. 


\title{
Temporal Processing in Patients with Tumor Invading the Supplementary Motor Area before and after Surgical Resection
}

\author{
Vincent Monfort ${ }^{1}$, Micha Pfeuty ${ }^{2,3}$, Hélène Brissart ${ }^{4}$ and Fabien Rech ${ }^{5,6}$ \\ ${ }^{1}$ University of Lorraine, LCOMS/2LPN-CEMA Group (Cognition-EMotion-Action), \\ Metz, France \\ ${ }^{2}$ University of Bordeaux, Bordeaux, France
}

${ }^{3}$ CNRS-UMR 5287 - Institut de Neurosciences Cognitives et Intégratives d'Aquitaine (INCIA), Bordeaux, France

${ }^{4}$ University Hospital of Nancy, Department of Neurology, Nancy, France

${ }^{5}$ Department of Neurosurgery, CHU Nancy, Nancy University Hospital, France

${ }^{6}$ Institut des Neurosciences de Montpellier, INSERM U1051, Montpellier, France

Based on a high number of functional magnetic resonance imaging and scalp electroencephalography studies, the supplementary motor area (SMA) has been proposed to play a key role in interval timing. However, only very few lesion studies have revealed difficulties in temporal processing in patients with SMA lesions and, furthermore, these studies were limited to motor timing. The aim of the present study was to determine whether the impact of SMA lesion on temporal processing depends on the motor component and on the range of duration under investigation. Two patients with tumor invading the SMA performed a set of several temporal tasks before and after (at few days and three months) surgical removal of the brain tumor: a color/duration discrimination task and a duration reproduction task in a short duration range (1200-1800 ms), verbal production and estimation tasks in a medium (4, 7, and $12 \mathrm{~s})$ as well as in a long (30 and $60 \mathrm{~s})$ duration range. Both patients underwent awake surgery and the removal of the tumor was performed according to the cortical and subcortical functional boundaries identified with electrical stimulation throughout the resection. The preSMA was resected in both patients and the SMA proper was resected only in patient 1 . Patient 1 was not investigated during the late post-surgical phase. Results revealed that, in early post-surgical phase, perceptual timing was impaired in both patients, and the impairment was more pronounced in the duration than in the color discrimination task. Motor timing was also disturbed but only in patient 1 . During the late post-surgical phase, timing performance of patient 2 increased compared to the early post- surgical phase and did not differ compared to the pre-surgical phase. These findings suggest that SMA resection results in an impairment of perceptual timing in early post-surgical phase, which would resolve in late post-surgical phase.

\section{Keywords}

Perceptual timing, motor timing, Supplementary Motor Area, surgery, tumor 


\title{
Intentional Binding as a Function of Action-Effect Interval and Semantic Relatedness
}

\author{
Penelope Bounia ${ }^{1}$, Vassilis Thanopoulos ${ }^{1,2}$ and Argiro Vatakis ${ }^{1,2}$ \\ ${ }^{1}$ Multisensory and Temporal Processing Laboratory (MultiTimeLab), \\ Department of Philosophy and History of Science, University of Athens, \\ Athens, Greece \\ ${ }^{2}$ Cognitive Systems Research Institute, Athens, Greece
}

Previous research have demonstrated that voluntary actions and their sensory effects are perceived closer in time; a phenomenon known as intentional binding (IB). Recently, Thanopoulos and Vatakis (submitted) have shown that IB occurs when voluntary actions and their effects hold an inherent causal link without the need for adaptation and when the effects follow in the predictable time interval of $250 \mathrm{~ms}$. Previous studies using abstract stimuli have demonstrated the induction of IB for action-effect intervals up to $650 \mathrm{~ms}$ and, in some cases $4000 \mathrm{~ms}$ (e.g., Humphreys \& Buehner, 2009). In our first experiment, therefore, we aimed to define the limits of IB maintenance in causal multisensory events as per Thanopoulos and Vatakis's study. We, thus, tested the participants in conditions varying in action intentionality and action-effect temporal predictability for intervals of 250, 800, 1000 and $1250 \mathrm{~ms}$ using a simultaneity judgment task. Analyses of the point of subjective simultaneity data showed that IB was observed only for the shorter intervals tested (i.e., 250 and $800 \mathrm{~ms}$ ), while no such effects were obtained for intervals over $1000 \mathrm{~ms}$. Additionally, no differences in discrimination performance were obtained for all intervals tested. These findings suggest that the causal link of naturalistic events is 'broken' when an effect is temporally far (i.e., $>1000 \mathrm{~ms}$ ) from its cause. Given the use of multisensory effects, our results, as well as the ones in Thanopoulos and Vatakis's study, may be affected by potential crossmodal binding rivalries (Kostaki \& Vatakis, 2016) between the action and the effect. Thus, our future studies will aim to further examine causal and temporal attraction of unequal sensory inputs in the IB phenomenon.

\section{Keywords}

Synchrony, multisensory, behavior, adult, 100s of ms-secs

\section{References}

Humphreys, G. R., \& Buehner, M. J. (2009). Magnitude estimation reveals temporal binding at super-second intervals.J. Exp. Psychol. Hum. Percept. Perform., 35, 1542-1549. Kostaki, M., \& Vatakis, A. (2016). Crossmodal Binding Rivalry: A "race" for integration between unequal sensory inputs. Vision Res., 127, 165-176.

Thanopoulos, V., \& Vatakis, A. (submitted). Robust intentional binding for causally-linked sequences of naturalistic events. 


\title{
Intentional Binding of Visual Effects
}

\author{
Andrea Kiesel, Miriam Ruess and Roland Thomaschke \\ Albert-Ludwigs-University of Freiburg, Germany
}

\begin{abstract}
When an action produces an effect, the action is perceived later in time compared to an action without following effect. Likewise, the effect of an action is perceived earlier in time compared to a similar stimulus without preceding action. This bias in time perception is called intentional binding (IB) and it serves as an implicit measure for sense of agency (e.g., Haggard, Clark, \& Kalogeras, 2002). One often used method to assess IB is to present a rotating clock hand to participants while they execute an action that produces a tone as effect stimulus. Participants estimate the point in time of either their action, or of the tone effect. These time estimates are compared to estimates from conditions in which an action or a tone occurs in isolation. Studies on IB commonly use auditory effects, whereas we modified the traditional paradigm by presenting visual effects. Firstly, we observed IB also for visual effects, showing that also our visual perception is subject to this temporal bias. Secondly, the size of the IB effect depended on the delay between action and effect. IB was strongest at delay ranges between $150 \mathrm{~ms}$ to $350 \mathrm{~ms}$, whereas (almost) no IB was found for delay ranges larger than $450 \mathrm{~ms}$. Thus, our results indicate that visual IB, that is, sense of agency for visual effects, might be maximal at different delays compared to auditory IB. This would have important implications for the design of human-machine interfaces depending on whether they use auditory or visual stimuli.
\end{abstract}

\section{Keywords}

Prediction, behavior, adult, Intentional Binding, time perception in action contexts, action-effect delay

\section{Reference}

Haggard, P., Clark, S., \& Kalogeras, J. (2002). Voluntary action and conscious awareness. Nature Neurosci., 5, 382-385.

\section{Intentional Binding of Two Effects}

\author{
Miriam Ruess ${ }^{1}$, Roland Thomaschke ${ }^{1}$, Carola Haering ${ }^{2}$, Dorit Wenke ${ }^{3}$ \\ and Andrea Kiesel ${ }^{1}$ \\ ${ }^{1}$ Albert-Ludwigs, Universität of Freiburg, Germany \\ ${ }^{2}$ Julius-Maximilians, Universität Würzburg, Germany \\ ${ }^{3} \mathrm{PFH}$ University of Applied Sciences, Göttingen, Germany
}

When an action produces an effect, the action is perceived later in time compared to an action without following effect. Likewise, the effect is perceived earlier in time compared 
to a stimulus without preceding action. Despite a substantial number of studies on this phenomenon - referred to as intentional binding effect (IB) - the underlying mechanisms are still not fully understood. Typically, IB is investigated in settings, where the action produces just one single effect, whereas, in everyday action contexts our actions rather cause sequences of effects before eventually leading to the desired outcome. Therefore, we accessed, in four experiments, IB of two consecutive auditory effects, instead of only one effect. We observed IB for the first tone, while time judgments for the second tone were only marginally biased. This pattern was observed for second tones that were temporally predictable (Experiment 1) or not (Exp. 2 and 3). In a fourth Experiment shorter effect delays were applied, because the duration of the effect delay has previously been shown to impact strongly on IB magnitude. Accordingly, the second tone yielded stronger IB when it was less delayed. Overall, our results provide a first indication that IB occurs for more than one single action effect. In situations similar to everyday action contexts, where our actions aim at producing sequences of effects, not just a first, but also a second action effect can be subject to IB. Yet, the results suggest that events occurring later in an unfolding action effect sequence might be bound less to actions than effects following actions directly. This, however, seems rather to be caused by the longer delay of a later occurring effect, instead of the fact, that it is the second one of two effects.

\title{
Keywords
}

Intentional binding, temporal binding, effect sequence, effect delay, action-effect interval, temporal predictability

\section{Causality as the Determinant Factor for Intentional Binding in Naturalistic Event Sequences}

\author{
Vassilis Thanopoulos ${ }^{1,2}$ and Argiro Vatakis ${ }^{1,2}$ \\ ${ }^{1}$ Department of Philosophy and History of Science, National and Kapodistrian \\ University of Athens, Athens, Greece \\ ${ }^{2}$ Cognitive Systems Research Institute, Athens, Greece
}

The temporal interval between an action and its consequent effect is subjectively perceived as shorter when the action is intentional and the effect is temporally predictable (Haggard et al., 2002). This phenomenon is commonly known as intentional binding (IB) with previous studies on IB focusing mainly on the use of abstract events (e.g., tones or flashes) with no expected causal link between the action and its effects. We, thus, investigated IB in multisensory causally-linked events through the production of the voluntary action of hitting a push-knob, while observing matching impact events on the computer screen (i.e., image-sound of a hand hitting a wooden surface). We designed a set of five experiments, where we manipulated intentionality (presence or absence of voluntary action), temporal predictability (fixed or random action-effect intervals), and causality (different action-effect causal relations), while participants completed a simultaneity 
judgment task (cf. Cravo et al., 2011). Our findings conflicted those of previous literature, revealing that voluntary action and temporal predictability alone are insufficient to generate IB in abstract audiovisual events (Exp. 1), without an adaptation/top-up task linking the action-effect. Absence of IB was also observed for cases where the naturalistic events presented were: a) without a causal linking of the whole action sequence of events (Exp. 2), b) causally mismatched, or c) mismatched in terms of response mapping (Exps. $4-5$, respectively). On the contrary, IB was reproduced when an enhanced causal linking was present between the fixation image and the subsequent multisensory effect (Exp. 3). That is, by simulating the whole impact movement of the hand, the impact effect was perceived closer in time to the voluntary action. Overall, our findings showed that causality is the necessary factor for the induction of IB and that the use of naturalistic events with inherent causality can lead to temporal action- effect compression without any adaptation procedures.

\title{
Keywords
}

Synchrony, multisensory, behavior, adult, 10s-100s of ms

\section{References}

Cravo, A. M., Claessens, P. M. E., \& Baldo, M. V. C. (2011). The relation between action, predictability and temporal contiguity in temporal binding. Acta Psychol., 136, 157-166.

Haggard, P., Clark, S., \& Kalogeras, J. (2002). Voluntary action and conscious awareness. Nat. Neurosci., 5, 382-385.

\section{A Motor Recalibration Influence on Color-Motion Asynchrony (CMA) Effect Size in a Visuo-Motor Paradigm}

\author{
Melisa Kurtcan and Inci Ayhan \\ Department of Psychology, Bogazici University, Turkey
}

Color-motion asynchrony (CMA) refers to an apparent asynchrony of visual features when a repeatedly oscillating moving dots change colour and motion direction simultaneously at the same rate (Moutoussis \& Zeki, 1997). It has been demonstrated that a single direction change is enough to induce CMA in cross-feature correspondence tasks (Linares \& Lopez-Moliner, 2006). Following a recent study, where Stetson et al. (2006) have shown a reversal effect in the subjective order of simultaneous action and sensation after subjects were adapted to a delayed sensory feedback regime, here, we examined whether such visuo-motor temporal recalibration is sensory-feature-specific and transferrable to multi-feature-stimuli in correspondence tasks such as CMA. We first measured the effect of visuo-motor recalibration in an experiment where subjects judged the temporal order of a voluntary key press and a change in the motion direction of an achromatic dot array 
following a delayed ( $100 \mathrm{~ms})$ sensory feedback adaptation in response to causal action. For those of subjects, who showed significant adaptation effects, we then conducted the main experiment using a single-direction-change CMA paradigm, where direction change was induced by voluntary key press and subjects were asked to report the predominant motion direction of target colour. Compared to baseline condition, we found that CMA was significantly reduced for the delayed sensory feedback adaptation condition. Control experiments showed that visuo-motor adaptation effects are not observed when the change in direction occurs independent of subject's key press, which together show a temporal recalibration effect in a visuo- motor CMA paradigm due to voluntary action. We show that visuo-motor temporal recalibration elicited with respect to a single visual feature (motion direction) in response to a voluntary causal action is transferrable to multi-feature-stimuli (i.e., chromatic dots changing motion direction) and reduce colour-motion asynchrony in a single-direction-change paradigm.

\title{
Keywords
}

100s of ms-secs, temporal order, duration, behavior, adult, temporal binding, causality

\section{References}

Linares, D., \& Lopez-Moliner, J. (2006). Perceptual asynchrony between color and motion with a single direction change. J. Vis., 6, 974-981.

Moutoussis, K., \& Zeki, S. (1997). A direct demonstration of perceptual asynchrony in vision. Proc. R. Soc. Lond. B, 264, 393-399.

Stetson, C., Xu C., Montague P. R., \& Eagleman, D. M. (2006). Motor-sensory recalibration leads to an illusory reversal of action and sensation. Neuron, 51, 651-659.

\section{Electrophysiological Correlates of Audiovisual Recalibration}

\section{Renan Schiavolin Recio, André Mascioli Cravo and Raphael Yokoingawa de Camargo}

\author{
Centro de Matemática, Computação e Cognição, Universidade Federal do ABC, Brazil
}

Several psychophysical studies have investigated how stimuli from different modalities are combined. Recently, there has been an increasing interest in how repeated exposure to a given delay between stimuli from two modalities can recalibrate subsequent temporal judgments (Fujisaki et al., 2004). However, the majority of these studies have focused on behavioral measures and the underlying neural mechanisms are still unclear. Here, we investigated the neural correlates of audiovisual recalibration. Human participants $(n=16)$ performed a variation of the stream-bounce task while we recorded EEG activity from 62 channels. In each trial, two discs moved towards each other and touched at a given time. Participants had to judge whether a beep was presented simultaneously with the moment that the discs touched. Before each experimental block, participants were exposed to a 
recalibration block in which the tone was presented either $235 \mathrm{~ms}$ before or after the discs touched. Consistent with previous results, we found that the point of subjective simultaneity (PSS) was modulated by previous exposure to the different delays. The EEG results suggested that theta oscillations in central-frontal electrodes were concentrated around different phases as a function of previous exposure. These results are consistent with the idea of low frequency oscillations playing in a key role in temporal framing.

\title{
Keywords
}

Audiovisual recalibration, EEG analysis, stream-bounce, multimodal integration

\section{Reference}

Fujisaki, W., Shimojo, S., Kashino, M., \& Nishida, S. Y. (2004). Recalibration of audiovisual simultaneity. Nat. Neurosci., 7, 773-778.

\section{Time Perception for Simple Stimuli: Effect of Inter- and Intra-Modal Stimulus Characteristics}

\author{
Vicky Karadima1, Maria Stamatelou ${ }^{2}$ and Argiro Vatakis ${ }^{1,3}$ \\ ${ }^{1}$ Multisensory and Temporal Processing Laboratory (MultiTimeLab), Department of \\ Philosophy and History of Science, National and Kapodistrian University of Athens, \\ Athens, Greece \\ ${ }^{2}$ Department of Psychology, National and Kapodistrian University of Athens, \\ Athens, Greece \\ ${ }^{3}$ Cognitive Systems Research Institute, Athens, Greece
}

Time estimation is depended on stimulus modality with visual stimuli often being judged as shorter than auditory stimuli (e.g., Ortega, Lopez, \& Church, 2009). However, very little is known about the effects of stimulus characteristics in timing with some studies showing gender-based temporal distortions in the presence of red-coloured stimuli (Shibasaki \& Masataka, 2014) and pitch-related temporal shifts for speech stimuli (Yu, 2010). In order to further explore modality and stimulus-quality effects on time perception, we asked participants to judge the presentation time (i.e., intervals from 400 to $1600 \mathrm{~ms}$ ) of simple stimuli via a bisection task. In terms of intra-modality, stimuli were presented as high- or low-pitch tones and black or white circles for the auditory and visual condition, respectively. The results revealed an underestimation for the visual stimuli as compared to the auditory stimuli. Furthermore, the weber ratio was higher in auditory as opposed to visual stimuli indicating poor discrimination sensitivity for the auditory stimulation. Looking at the intra-modal characteristics, we found that both the lighter color and the lower pitch stimuli resulted in an interval underestimation as compared to the darker color and higher pitch stimulation, respectively. The temporal sensitivity, as revealed by the weber ratio analysis, was lower for the color comparisons with darker stimuli resulting in lower sensitivity as compared to light-colored stimuli. No significant sensitivity differences were 
found for the high and low pitch tones presented. Taken together, our findings suggest that timing may be depended on the stimulus characteristics rather than stimulus modality alone, thus making the study of stimulus qualities in timing critical.

\section{Keywords}

Modality, duration, behaviour, adult, 10s-100s of ms

\section{References}

Ortega, L., Lopez, F., \& Church, R. M. (2009). Modality and intermittency effects on time estimation. Behav. Processes, 81, 270-273.

Shibasaki, M., \& Masataka, N. (2014). The color red distorts time perception for men, but not for women. Sci. Rep., 4, 5899.

Yu, A. C. (2010). Tonal effects on perceived vowel duration. Lab. Phonol., 10, 151-168.

\section{Are There Modality-Specific Effects of Preceding Flicker and Flutter on the Reproduction of Visual and Auditory Durations?}

\section{Karin M. Bausenhart and Maria D. De la Rosa}

University of Tübingen, Germany

Previous research showed that the perceived duration of temporal intervals is prolonged when visual flicker or auditory flutter precedes these intervals. Moreover, this effect of "pacing" stimulation increases with increasing interval duration (e.g., Penton-Voak et al., 1996; Wearden et al., 1998). Within the pacemaker-accumulator model of time perception, this is interpreted as an acceleration of the pacemaker rate caused by the pacing stimulation. In two experiments, we investigated whether this effect depends on the congruency of pacing stimulation (visual/auditory/no pacing) and interval modality (visual/auditory). If pacing stimuli and temporal intervals are processed by a single, modality-independent pacemaker, modality congruency should not alter the magnitude of the pacing effect. In contrast, if stimulation from different modalities is processed by separate pacemaker modules, the magnitude of the pacing effect should vary with modality congruency. In line with previous findings, pacing stimulation prolonged perceived duration compared to no pacing stimulation, and this effect increased with increasing stimulus duration. Importantly, this interaction was independent of pacing modality and interval modality, suggesting that all stimuli are processed by a single, modality-independent pacemaker. Furthermore, when interval modality was varied blockwise rather than trial-by-trial, an additional modalityspecific influence of pacing was observed. Specifically, congruent combinations of pacing and interval modality produced slightly longer durations than incongruent combinations. In contrast to the overall effect of pacing vs. no pacing, this effect did not increase with interval duration. Therefore, we attribute this effect to modality- based attention (Mattes \& 
Ulrich, 1998). Presumably, modality-congruent pacing stimulation facilitates attentional selection of the subsequently presented target interval, and thereby reduces the latency of the switch component of the pacemaker- accumulator model.

\title{
Keywords
}

Duration, multisensory, behavior, adult, 100s of ms-secs

\section{References}

Mattes, S., \& Ulrich, R. (1998). Directed attention prolongs the perceived duration of a brief stimulus. Percept. Psychophys., 60, 1305-1317.

Penton-Voak, I. S., Edwards, H., Percival, A., \& Wearden, J. H. (1996). Speeding up an internal clock in humans? Effects of click trains on subjective duration.J. Exp. Psychol. Anim. Behav. Process., 22, 307-320.

Wearden, J. H., Edwards, H., Fakhri, M., \& Percival, A. (1998). Why "sounds are judged longer than lights": Application of a model of the internal clock in humans. Q. J. Exp. Psychol. B, 51, 97-120.

\section{The Effect of Modality Congruency in Isochronous Sequences}

\author{
Min Susan Li and Massimiliano Di Luca \\ Centre for Computational Neuroscience and Cognitive Robotics, School of Psychology, \\ University of Birmingham, UK
}

Previous work highlighted that the precision with which we can discriminate isochrony in auditory sequences improves with longer sequences, according to the predictions of computational models of perception (Li, Rhodes, \& Di Luca, 2016). Despite the increase in precision, there is an associated decrease in accuracy, which is captured by the prediction of prior entry, where attentional facilitation speeds up perception (Sternburg \& Knoll, 1973). In this work, we explore whether these effects are present with sequences composed of cross-modal stimuli. We presented five isochronous stimuli (either audio or visual) followed by a sixth anisochronous stimulus (either audio or visual) to create either congruent (e.g., A-A-A-A-A-A) or incongruent sequence conditions (e.g., A-A-A-A-A-V). We presented the four conditions resulting from the combination of the two modalities either in separate blocks or interleaved. Participants were asked to report whether the final stimulus was earlier or later than the expected timing. We found that visual stimuli led to less precise answers than audio, especially when comparing the last stimulus. An incongruent last stimulus led to even worse isochrony discrimination performance. Surprisingly, we didn't find a decreased performance with interleaved presentation. Notably, results also indicated a latency difference between auditory and visual perception. In sum, our results suggested different temporal mechanisms for auditory and vision, and illustrated the cross-modal interaction in an isochronous sequence, based on the sequence build-up and the stimulus to be judged. 


\title{
Keywords
}

Rhythm, synchrony, multisensory, modelling, 10s-100s of ms

\section{References}

Li, M. S., Rhodes, D., \& Di Luca, M. (2016). For the last time: Temporal sensitivity and perceived timing of the final stimulus in an isochronous sequence. Timing Time Percept., 4, 123-146.

Sternberg, S., \& Knoll, R. L. (1973). The perception of temporal order: Fundamental issues and a general model. Attention and Performance, IV, 629-685.

\section{Modality Differences in Timing: Testing the Pacemaker Speed Explanation}

\author{
Emily A. Williams, Andrew J. Stewart and Luke A. Jones
}

The University of Manchester, UK

We investigated the classic effect that "sounds are judged longer than lights" when the two are of equal duration (Goldstone et al., 1959). Recent work has found that durations of vibrations are judged somewhere between the two (Jones et al., 2009). This pattern has also been found for temporal difference thresholds, where sensitivity is highest for sounds, followed by vibrations, and lowest for lights. These findings have been interpreted within Scalar Expectancy Theory as due to a central pacemaker, which runs at a faster rate for sounds, followed by vibrations, and slowest for lights (Wearden et al., 1998). The current work aimed to test this assertion and investigate two alternative explanations. As both estimates and thresholds are said to depend on the pacemaker, it is reasonable to expect some level of correlation between them. However no significant correlations were found. Additionally we investigated whether participant's best-performing modality was the same in both tasks, though this was not the case. Therefore, the assertion that pacemaker speed is the determining factor in performance for these tasks is called into question. A second study investigated whether the modality differences could instead be explained by participants' sensory biases. The same participants completed cross- modal temporal order judgments, and sensory bias was measured by their point of subjective simultaneity. No correlations were found between the differences in estimation or thresholds and their respective sensory biases. This suggests that modality differences may not be due to sensory biases. Finally, we used computer modelling to investigate whether the differences between modalities in estimates and thresholds could be alternatively explained by differences in the variability of the stored internal representation. The simulations found that increasing internal variability was not in itself sufficient to cause the differences.

\section{Keywords}

Pacemaker, multisensory, estimates, thresholds, bias, variability 


\title{
References
}

Goldstone, S., Boardman, W. K., \& Lhamon, W. T. (1959). Intersensory comparisons of temporal judgments. J. Exp. Psychol., 57, 243-248.

Jones, L. A., Poliakoff, E., \& Wells, J. (2009). Good vibrations: Human interval timing in the vibrotactile modality. Q.J. Exp. Psychol., 62, 2171-2186.

Wearden, J. H., Edwards, H., Fakhri, M., \& Percival, A. (1998). Why "sounds are judged longer than lights": Application of a model of the internal clock in humans. Q. J. Exp. Psychol. B, 51, 97-120.

\section{On the Stability of Temporal Reference Information in Duration Discrimination}

\author{
Ruben Ellinghaus, Karin M. Bausenhart and Rolf Ulrich \\ University of Tübingen, Tübingen, Germany
}

Psychophysical research suggests that duration judgments do not only depend on the current interval duration, but are also influenced by the duration of previously presented intervals. The Internal Reference Model (IRM) explains this effect by assuming that participants rely on an internal reference for their duration judgments. Specifically, the internal reference consists of both previous and current stimulus instances and updates continuously from trial to trial. Interestingly, IRM predicts better duration discrimination performance when a constant standard precedes rather than follows a variable comparison, a pattern of results which is referred to as the Type B effect and which is usually observed in two-alternative-forced-choice (2AFC) discrimination. The present experiments assess the stability of the internal reference by means of its susceptibility to the presentation of distractor intervals during the intertrial interval (ITI). In detail, participants discriminated between two successively presented intervals marked by continuous $500 \mathrm{~Hz}$ sine tones. When white noise distractors were presented during the ITI, a similar Type B effect was observed as when no distractors were presented. In contrast, the Type B effect increased when $500 \mathrm{~Hz}$ sine tones were presented as distractors. Within IRM, there are two possible causes for this pattern of results. First, the distractor might be integrated into the internal reference. Second, the distractor might interfere directly with the perception of the first task-relevant interval and thus cause participants to attach a higher weight to previously presented intervals when forming the internal reference. In any case, perceptual similarity seems to be an important modulator of this effect, which might indicate that the internal reference contains specific stimulus features rather than merely abstract temporal information.

\section{Keywords}

Duration discrimination, Internal Reference Model, psychophysics, temporal memory 


\title{
Does Imagined Stimulus Size Influence Duration Judgments in the Same Way as Physical Stimulus Size Does?
}

\author{
Rolf Ulrich and Teresa Birngruber
}

University of Tübingen, Germany

Previous studies have shown that physical stimulus size influences perceived duration. Larger stimuli tend to be judged as lasting longer than smaller ones (e.g., Thomas \& Cantor, 1975). Similar effects can be observed when stimuli only appear to be of different size because of a size illusion but are actually of the same physical size (Ono \& Kawahara, 2007). If not objective stimulus size drives the size effect on perceived duration, stimuli which only differ in imagined size might elicit this effect as well. Classic studies of mental imagery have shown that participants imagine things "true to real size", for example an elephant as being larger than a mouse (Kosslyn, 1975). Accordingly, we investigated whether the duration of words representing small or large animals would be judged differently. In two experiments we presented names of small or large animals (matched for number of letters and word frequency) for 700, 1000 or $1300 \mathrm{~ms}$. Participants were asked to imagine the animals in front of a constant background (on a tennis court) and to reproduce the presentation duration by holding down a button for the same duration. In Experiment 1, participants additionally had to judge the animals' size in 10\% of trials; in Experiment 2 they only performed the reproduction task. The durations of words representing large animals were reproduced longer than those of words representing small animals, but only if animal size was judged as well (Experiment 1). A third experiment is currently running to test the reliability of the effect observed in Experiment 1 . The findings suggest that imagined stimulus size can influence time perception under certain circumstances, namely when size differences are made salient.

\section{Keywords}

Duration, language, behavior, adult, ms-secs

\section{References}

Kosslyn, S. M. (1975). Information representation in visual images. Cogn. Psychol., 370, 341-370.

Ono, F., \& Kawahara, J.-I. (2007). The subjective size of visual stimuli affects the perceived duration of their presentation. Percept. Psychophys., 69, 952-957.

Thomas, E. A. C., \& Cantor, N. E. (1975). On the duality of simultaneous time and size perception. Percept. Psychophys., 18, 44-48. 


\title{
Non-Gaussian Representation of the Subjective Time
}

\author{
Marcelo Bussotti Reyes ${ }^{1}$, Tomás Gallo Aquino ${ }^{2}$ and Raphael Yokoingawa \\ de Camargo ${ }^{1}$ \\ ${ }^{1}$ Center for Mathematics, Computation and Cognition, Universidade Federal do ABC, \\ Brazil \\ ${ }^{2}$ Computation \& Neural Systems, California Institute of Technology, USA
}

Internal (subjective) time is frequently represented as a Gaussian distribution. One drawback of this approach is that Gaussian assumes negative values, which may be incompatible with some experimental results. Also, Gaussian distributions do not reproduce the skewness frequently observed in some experimental results, as for example the fixed interval procedure. Hence, distributions whose domain is exclusively non-negative might be better candidates for the description of the subjective time. We investigated whether the inverse Gaussian and log-normal probability distribution can be suitable in such description. Through a change of parameters, we derived analytical forms of the distributions that are compatible with the scalar property. We also showed that the two candidate distributions approach each other for small (but still in an experimental range) Weber fractions. Furthermore, we show that the distributions replicate the classical result of the temporal bisection procedure, in which bisection times equal the geometric mean of reference intervals. These results suggest that not only are log-normal and inverse-Gaussian suitable for internal time representation, but they may be virtually experimentally indistinguishable.

\section{Keywords}

Duration, behaviour, modeling, simulated data, ms-sec

\section{The Neuroscience of Apparent Motion: Towards a Theory of Temporal Experience}

\author{
Matthew Stuart Piper \\ UC San Diego, USA
}

I will be presenting a neurobiological model of the sub-second timing mechanisms responsible for explaining the temporal illusion known as apparent motion, which is the most important case study in philosophical debates about temporal experience. This debate has recurrently been characterized as a fight among three views: the cinematic view, retentionalism and extensionalism. My goal is to use a neuroscientifically detailed explanation of apparent motion as a lever to make a larger claim about the most warranted theory of temporal experience. Apparent motion is commonly experienced when two spatially proximal lights flash with $\sim 60-120$ msec delay between them. Instead of two 
stationary flashes, a moving light is experienced. Many philosophers have argued that this shows that the timing of brain dynamics cannot help explain temporal representation and experience (Grush, 2007). If that's correct, the theory of temporal experience known as extensionalism is false. The neural circuitry model I will present is an elaboration of work done by Valteri Arstila (2016), who posits a "non-linear latency difference" explanation for apparent motion. My model adds a theory of the basic neurodynamical organization required for representational content, which I argue to be certain forms of cross-frequency coupling - an idea gaining traction in the neuroscience community (Gu et al., 2015). I present a detailed model of timing dynamics at the msec scale that accounts for the experimental data but does so in a manner congruent with extensionalism. The upshot of my work is a defense of the view that temporal representation and experience is explained by the temporal organization of key neurodynamical circuits. This is a direct defense of the theory of extensionalism and against the rival theories of retentionalism and the cinematic view.

Keywords

Rhythms, neuropsychology, adult, 100s of msec, temporal illusion, multiplexing

\title{
References
}

Arstila, V. (2016). The time of experience and the experience of time. In B. Molder et al. (Eds), Philosophy and psychology of time, Studies in Brain and Mind 9.

Grush, R. (2007). Time and experience. In T. Muller (Ed.), The philosophy of time. Frankfurt: Klosterman.

Gu, B., van Rijn, H., \& Meck, W. (2015). Oscillatory multiplexing of neural population codes for interval timing and working memory. Neurosci. Biobehav. Rev., 48, 165-180.

\section{Demixing the Perception of Duration and Intensity in Vibrotactile Behavior}

\author{
Alessandro Toso, Arash Fassihi, Francesca Pulecchi, Sara Sorella \\ and Mathew E. Diamond
}

International School for Advanced Studies, Trieste, Italy

We investigated how human subjects and rats can flexibly extract both duration and intensity from a single vibrotactile stimulus. On every trial, subjects received two vibrations Stimulus1 of duration T1 and Stimulus2 of duration T2. In Experiment 1, both rats and humans learned to compare either (i) the relative mean speeds of Stimulus1 and Stimulus2, or else (ii) their relative durations T1 and T2. In Experiment 2, human subjects had to estimate either the duration or the intensity of a single vibration by scaling their judgment through a software slider. In both experiments, perceived duration was dependent on stimulus intensity while, symmetrically, perceived intensity was dependent on 
stimulus duration. We developed a behavioural model that explains the effects of stimulus intensity and duration on perceived intensity and duration as a non-linear temporal summation of instantaneous speed. To test our model through experiments, we introduced ramping stimuli, where the original vibration was multiplied by a linear sloping function, while conserving its mean speed. Human and rat subjects judged ramping-up stimuli as longer in duration, but weaker in intensity; ramping-down stimuli as shorter in duration, but stronger in intensity. These results demonstrate that duration perception is modulated by the integration of vibration speed values following an exponentially growing function, while intensity perception follows an exponentially decaying function. Moreover we searched for the neuronal signatures of the computations inherent to the duration- intensity confound. Neuronal activity was recorded in behaving rats, during both an intensity and a duration discrimination task, from both barrel cortex and premotor cortex. Results are now being analyzed. Overall our findings support the idea that the representations of stimulus duration and stimulus intensity are intermixed in the brain. While both percepts arise from the accumulation of vibration events, the temporal profile of accumulation changes according to the behavioural task.

\title{
Keywords
}

Duration, behaviour, adult, rats, tactile perception, 100s of ms

\section{Constructing the Perceived Unit of Time}

\author{
Gerardo Viera $^{1,2}$ \\ ${ }^{1}$ University of British Columbia, Department of Philosophy, Vancouver, Canada \\ ${ }^{2}$ University of Antwerp, Centre for Philosophical Psychology, Antwerp, Belgium
}

Even though we acquire information about the temporal structure of events spanning multiple timescales (from milliseconds through multiple seconds) through multiple sensory systems, we do not perceive the world as consisting in multiple distinct timelines. Instead, the various events we perceive through the multiple sensory systems are perceived as occurring within a single unified temporal order. It is in this way that time is unified as we perceive it. Within the cognitive sciences and philosophy there are two dominant approaches to explaining the perceived unity of time - the centralized-clock models and models the posit a matching between the temporal contents of perception and the temporal structure of perceptual processes. These approaches assume that an explanation of how perception carries information about time will also explain the perceived unity of time. I argue that these accounts fail. Our capacity to perceive time is not a single homogenous psychological capacity. The existence of various dissociations between different timekeeping capacities shows that the ability to perceive time is composed of a number of distinct capacities to perceive the temporal structure of events in our environment. Importantly, the various timekeeping mechanisms that contribute temporal information to perception 
carry information in radically different coding schemes (e.g., a combination of intrinsic and various extrinsic timekeeping mechanisms). As a result, the standard explanations of the perceived unity of time fail. Instead, the problem of explaining the perceived unity of time should be construed as a translation problem, which parallels the establishment of a system of standardized units in the history of science. I conclude the poster by drawing out the parallels between the translation problem the brain faces and an episode in the history of thermometry. In both cases, the establishment of standardized-units can explain how various measurement devices could be interpreted within a single common-code.

\title{
Keywords
}

Multisensory, philosophy, multiple timescales, neuropsychology, adult, experience

\section{Unified Mechanisms for Simultaneous Processing of Physical Properties and Subsecond Time Parameters of Sensory Stimuli in the Same Neural Circuits}

\author{
I. G. Silkis \\ Institute of Higher Nervous Activity and Neurophysiology of the Russian Academy of \\ Sciences, Moscow, Russia
}

A unified mechanism for the simultaneous processing of physical properties of sensory stimuli and their duration in subsecond time scale is suggested. It is based on the assumption that there is no necessity to determine time parameters of sensory stimuli regardless of their physical properties. All information is processed in the following parallel associative and limbic neural circuits: neocortex (basolateral amygdala) - basal ganglia thalamus - neocortex (basolateral amygdala), and neocortex - subthalamic nucleus pedunculopontine nucleus - thalamus - neocortex. Processing is similar for stimuli of different sensory modalities owing to resemblance of functioning of topically organized cortico - basal ganglia - thalamocortical loops. Time counting is started involuntary by a stimulus, or voluntary due to activation of prefrontal cortex. Time estimation is based on the clock rate of information processing that is inversely proportional to the duration of one cycle of repeated excitation of the neocortex (about $25 \mathrm{~ms}$ ). This excitation is provided by circulation of activity in mentioned neural circuits. Perceived time is proportional to the number of cycles of circulation. Clock rate depends on the strength of a stimulus, its emotional significance, attention, and current concentrations of neuromodulators. It follows from proposed unified mechanism that any factor reinforcing (weakening) the initial neocortical representation of physical properties of a stimulus and causing an increase (decrease) in the efficacy of cortico-striatal inputs, should facilitate (hinder) the disinhibition of the thalamus through the basal ganglia and its excitation by pedunculopontine nucleus. Subsequent decrease (increase) in duration of activity circulation in considered 
neural loops will cause a rise (fall) of clock rate, and overestimation (underestimation) of perceived time. This mechanism can serve the neurophysiological basis for recently proposed unified principle of sensory processing.

\section{Keywords}

Prediction, multisensory, modeling, neurophysiology, 100s of ms-secs

\section{Integrating Temporal Control Models on the Grounds of a Computational Architecture}

\section{Estêvão Gonçalves Bittar and José Lino Oliveira Bueno}

University of São Paulo, Faculty of Philosophy, Sciences, and Letters of Ribeirão Preto, Department of Psychology, Section of Psychobiology, Center for Experimental Aesthetics, Ribeirão Preto, Brazil

The basic models of MPR are used as the building blocks of a stochastic machine that simulates operant responding. The MPR-machine is then tested under a wide variety of experimental conditions, and proves its ability to produce realistic (a) IRT distributions, (b) within-session patterns of responding, and (c) relations between reinforcement rate and response rate under interval and ratio schedules. In this study, the MPR-machine is embodied with a model of temporal control (Machado et al., 2009). The new configuration, named tMPR, proves itself able to simulate (f) the timing behavior that arises under predictable interval schedules as well as $(\mathrm{g})$ the central properties of this behavior. The power to account for a vast domain of operant behavior phenomena with a simple mechanism that runs on a fixed set of parameter values - and the fact that it does so based on an architecture that can be easily expanded to accommodate new models - makes the MPR machine a useful platform for theory integration. As other researchers enrich the framework with new modules, it may elucidate how different aspects of operant behavior interact with one another, paving the transition from a behavior analysis to a behavioral synthesis. Finally, open source software that implements the MPR-machine is provided for those interested in contribute to the architecture evolution.

\section{Keywords}

Duration, behaviour, modelling, animal (rats), secs-mins, arousal

\section{Reference}

Machado, A., Malheiro, M. T., \& Erlhagen, W. (2009). Learning to time: A perspective. J. Exp. Anal. Behav., 92, 423-458. 


\title{
Using Time to Enhance Fluent Human-Robot Interaction
}

\author{
Michail Maniadakis and Panos Trahanias \\ Foundation for Research and Technology Hellas (FORTH), Greece
}

The long-term symbiotic interaction between humans and robots has tremendous potential, as the robots bond with people, providing significant assistance in their daily activities. Despite the increasing research endeavors devoted to human-robot synergies, we still know little about creating systems that function smoothly and effectively within the context of prolonged companionships. Contemporary research has mainly concentrated on enhancing relevant sensory, perceptual and motor capacities, assuming short-term and nearly momentary interaction between the two ends. Still, human-machine confluence encompasses an inherent temporal dimension that is typically overlooked by current research projects. We identify three important disciplines of cognition in which time has a major role: (i) long, mid and short-term action planning, (ii) active perceptuo-motor coordination and real-time interaction with the environment, (iii) self-experiences representation and memorization on a temporally rich domain. Broadly speaking, these disciplines cover the three main temporal views that living organisms develop on the world, namely the future, the present and the past, all three of them being essential for effectively addressing the long-lasting and continuous aspects of human-robot symbiotic interaction. For a number of years, FORTH has proposed and developed modules that contribute in implementing "entimed" robotic cognitive systems. Recent computational advances over all three disciplines will be summarized and discussed in the current paper, with emphasis on the interactions and the mutual support they provide to each other. We envision the development of a new generation robotic systems that exploit temporal cognition to seamlessly integrate into the heavily time-structured human societies.

Keywords

Multisensory, modeling, mins-hours, simulated data, human-robot interaction

\section{Examining the Concept of Temporality in Information System Development Flow}

\author{
Mairead O' Connor and Kieran Conboy \\ Lero, The Irish Software Research Centre, National University of Ireland, Galway, Ireland \\ There is strong evidence to suggest that awareness and use of flow information systems \\ development (ISD) techniques are gaining popularity across the ISD community. The key
}


differentiating feature of flow methods is their emphasis on sustainable speed of software development and delivery, epitomised by terms such as lead time, cycle time, queue time, cost of delay, burndown rate, and velocity. Despite this emphasis on speed, little research on ISD flow considers the complexity of time. Time can be classified in many ways (e.g., clock time, event time, non-linear time), it is socially constructed in different ways across different individuals, teams, organisations and locations. It can be mapped to development events in many ways. It is also subject to different temporal perceptions and personalities amongst members of the ISD team. Most existing ISD research generally, and certainly ISD flow research specifically, adopts a very simplistic, 'clock- based' mechanistic interpretation of time and ignores the highly complex, multi-faceted, subtle and socially embedded nature of temporality. This is a significant limitation given that ISD is a highly complex, socially embedded activity. This research applies temporality theory (Ancona et al., 2001) to examine ISD flow in three companies, examining not just clock time, but broader temporal concepts such as event time, temporal perception, temporal personality, and synchronicity. This research contributes to research and practice by (i) identifying the temporal characteristics of flow practices; (ii) identifying temporal challenges of flow practices; (iii) identifying strategies for resolution and; (iv) developing an extensive roadmap for future research to address the current dearth of solutions in existing literature. While this study examines flow methods, lessons learned can then be tailored and applied to other ISD contexts.

\title{
Keywords
}

Duration, rhythms, synchrony, behaviour, adult, mins-hours

\section{Reference}

Ancona, D. G., Okhuysen, G. A., \& Perlow, L. A. (2001). Taking time to integrate temporal research. Acad. Manage. Rev., 26, 512-529.

\section{The Haptic of Time}

\author{
Luca Ferraro $^{1}$ and Laura Rossi ${ }^{2}$ \\ ${ }^{1}$ Re:Lab, Reggio Emilia, Italy \\ ${ }^{2}$ Altran Spa, Torino, Italy
}

We investigated how long a prolonged interaction with a switch should be in order to be estimated as a "long" interaction. Long press for physical or virtual buttons is widely used in user interaction design, from the automotive to mobile phone fields, for example as a strategy to activate particular functions or delete some contents. Surely, the feedback that the interaction is successfully completed plays a fundamental role, but what is the minimum duration of an interaction that is supposed to be perceived as a long one? In an experiment, 25 participants pressed a button for a time they estimated to be adequate for a long button press in order to activate a supposed function. No feedback was given. 
Moreover, in a second part of the experiment participants estimated different button press durations (feedback was given as signal of the activation of the function) and ranked them by preference. In the lack of a feedback, users underestimated the proper duration of a long interaction. However, they chose a precise duration as preferred when a feedback was given. This duration was significantly longer than the one produced in the first part of the experiment. Prolonged interaction is a very useful strategy for a lot of tasks, however no substantial data are shown in literature to understand how a long this kind of interaction should be. For user-interaction design, this study shows what is the duration that is perceived as the most adequate for a long interaction. Moreover, timing and feedback associated with key interaction highlighted the fundamental role of the feedback not only in making the user understand that the action is completed, but also in the subjective evaluation of the duration of an interaction.

\title{
Keywords
}

Duration perception, behaviour, adult, 100s of ms-secs

\section{References}

Branaghan, R. J., \& Sanchez, C. A. (2008). Feedback, subjective time estimates and user satisfaction. Proceedings of the Human Factors and Ergonomics Society Annual Meeting.

Kelty, S., Damian, G., \& Dixon, J. A. (2014). Interwoven fluctuations during intermodal perception. J. Exp. Psychol. Hum. Percept. Perform., 40.

Meyer, J., Shinar, D., Yuval, B., \& Leiser, D. (1996). Duration estimates ad users' preferences in human computer interaction. Ergonomics, 39, 46-60.

\section{Time Perception in Human-Computer Interaction: The Role of Duration Perception and Passage of Time in User Experience}

\author{
Anna K. Trapp \\ Technische Universität Berlin, Germany
}

User experience (UX) has been investigated extensively in human-computer interaction (HCI) during the last two decades and can be described as the user's perceptions and responses resulting from the use of a device. The perceptions comprise perceived hedonic and pragmatic qualities. In a series of four experiments we investigated the connection between time perception and these components of UX. More precisely, we focused on prospective verbal duration estimates and passage of time judgements (PoTJs, time went by fast vs. slow) within the range of typical HCIs (30s to $10 \mathrm{~min}$ ). We made four major observations. First, duration estimates were distorted as expected by increased workload. Second, PoTJs were not affected by workload but by features of the device. Third, PoTJs 
showed moderate correlations with perceived pragmatic qualities but only small correlations with perceived hedonic qualities of the device. Fourth, manipulating passage of time caused changes in perceived pragmatic and hedonic qualities. In the first two experiments we experimentally manipulated pragmatic qualities of the device and workload by a secondary task. As expected higher compared to lower workload diminished duration estimates, while pragmatic qualities only affected PoTJs (Observation 1 \& 2). Correlations (Observation 3) were also found in first two experiments and replicated in Experiment 3 in which we used original websites with differing pragmatic and hedonic qualities instead of an experimental manipulation. In Experiment 4 we manipulated passage of time (time is flying by vs. dragging). Ratings on hedonic and pragmatic qualities were higher in the 'flying by' compared to the 'dragging' condition (Observation 4). We conclude that considering temporal aspects in HCI enhances the understanding of UX. Moreover, the findings emphasize the relevance of PoTJs to further understand humans' ability to perceive time at different levels of abstraction and to employ this understanding in an applied context.

Keywords

Duration, behavior, adult, secs-mins, passage of time judgments

\section{Decisional, Attentional and Working Memory Mechanisms in Generalisation and Bisection Tasks: An ERP Study}

\section{Dorian Bannier, Robert Lalonde and Mohamed Rebaï}

Normandie Univ, UNIROUEN, CRFDP, Rouen, France

The aim of this study was to determine how decisional, attentional and working memory mechanisms are modulated differently in generalisation and bisection temporal tasks. The generalisation consists in indicating whether a presented duration was a standard duration previously learned. In the bisection paradigm, subjects had to tell if the presented durations were closer to a short or a long previously learned standard duration. Participants were exposed to visual durations ranging from 200 to $1400 \mathrm{~ms}$. Processes of interest were the comparison between presented duration and standard duration(s) held in memory, decision- making, orientation of attention and context updating in working memory, indexed respectively by a frontopolar positive slow wave (PSW), the LPCt, the P3a and the P3b, by means of event-related potentials. The study revealed three main results. First, the paradigm affected only the orientation of attention (P3a): cerebral activity was ampler in the generalisation task than in the bisection task for short durations (200-800 $\mathrm{ms}$ ), indicating that the generalisation task may be more difficult than the bisection task. Secondly, the short durations (200 ms and $400 \mathrm{~ms}$ ) had an impact on decision-making (LPCt), orientation of attention (P3a) and context updating in working memory (P3b) 
independently of the paradigm concerned. These results are considered to reveal a general boost of attention resource allocation in favor of the shorter durations, because these intervals are too short to be effectively processed during the stimulus subtending it. Thirdly, the comparison mechanism could be indexed on ERPs by the PSW only during longer durations (800-1400 ms), revealing the presence of a frontal timing mechanism specific to long durations. Taken together, these results allow better understanding of the dynamics of temporal processing in duration discrimination tasks.

\title{
Keywords
}

Duration, behaviour, electrophysiology, adult, 100s of ms-secs

\section{Decoding the Representation of Time in Brain States Using Multivariate Pattern Analysis}

\author{
Vanessa Carneiro Morita, Fernanda Dantas Bueno, \\ Raphael Yokoingawa de Camargo, Marcelo Bussotti Reyes, \\ Marcelo Salvador Caetano and André Mascioli Cravo \\ Centro de Matemática, Computação e Cognição, Universidade Federal do ABC (UFABC), \\ Brazil
}

A growing number of studies have started to focus on brain dynamics as a mechanism for temporal encoding. Although there is growing evidence in favour of this view from computational and in vitro studies, there is still a lack of results from experiments in humans. In this study we show that high-dimensional brain states revealed by multivariate pattern analysis of human electroencephalography (EEG) are correlated to temporal judgments. Participants performed a temporal categorisation task based on a "shoot the target" task previously used to study temporal perception (Guilhardi et al., 2010). In our analysis, we first show that, as participants estimate temporal intervals, the spatiotemporal dynamics of their brain activity are consistent across trials. Second, we present evidence that these dynamics exhibit properties of temporal perception, such as scale invariance. Lastly, we show that it is possible to predict temporal judgments based on these brain states recovered from EEG. These results show how scalp recordings can reveal the spatiotemporal dynamics of human brain activity related to temporal processing.

\section{Keywords}

Duration, behaviour, EEG, adult, 100s of ms-secs

\section{Reference}

Guilhardi, P., Menez, M., Caetano, M. S., \& Church, R. M. (2010). The effect of stimulus discriminability on strategies for learning multiple temporal discriminations. Behav. Processes, 84, 476-483. 


\title{
An Intracranial Electroencephalography Investigation of the Supplementary Motor Area in the Reproduction of Supra-Second Time Intervals
}

\author{
Micha Pfeuty ${ }^{1,2}$, Vincent Monfort ${ }^{3}$, Madelyne Klein ${ }^{3}$, Julien Krieg4, \\ Steffie Collé ${ }^{3}$, Jean- Pierre Vignal ${ }^{4,5}$, Jacques Jonas ${ }^{4,5,6}$ \\ and Louis Maillard 4,5,6 \\ 1University of Bordeaux, INCIA, UMR 5287, Bordeaux, France \\ ${ }^{2}$ CNRS-UMR 5287 - Institut de Neurosciences Cognitives et Intégratives d'Aquitaine \\ (INCIA), Bordeaux, France \\ ${ }^{3}$ University of Lorraine, LCOMS/2LPN-CEMA Group (Cognition-EMotion-Action), Metz, \\ France \\ ${ }^{4}$ Université de Lorraine, CRAN, UMR 7039, Campus Sciences, Vandoeuvre-lès-Nancy, \\ France \\ ${ }^{5}$ University Hospital of Nancy, Department of Neurology, Nancy, France \\ ${ }^{6}$ Faculté de Médecine de Nancy, Université de Lorraine,Vandoeuvre-lès-Nancy, France
}

The supplementary motor area and (SMA) and the anterior insular cortex/ inferior frontal gyrus (AIC/IFG) have been shown be involved in interval timing but their precise role remains a matter of debate. The present study aimed at examining, by the means of intracranial EEG recordings, the time course of the activity in these two structures, as well as in other cortical areas, during a visual time reproduction task. Five epileptic patients with depth electrodes implanted into the SMA and/or the AIC/IFG region, had to encode, keep in memory and then reproduce the duration $(3,5,7 \mathrm{~s})$ of emotionally-neutral or negative pictures. Event-related potentials (ERPs) were analyzed during three periods: At the beginning and at the extinction of the target interval (TI) and at the beginning of the reproduction interval (RI). Electrophysiological data revealed an ERP time-locked to TI-offset whose amplitude decreased monotonously with TI-duration. This effect was observed in three patients and concerned mainly the SMA and less consistently the insular, frontal and cingulate cortical regions. Furthermore, a time-frequency (TF) analysis revealed in the same cortical regions concomitant increases in gamma-band $(40-80 \mathrm{~Hz})$ and decreases in alpha-band $(8-13 \mathrm{~Hz})$ activities which were also inversely proportionate to TI-duration. In contrast, modulations of ERPs with emotion or with trial-to-trial fluctuations of temporal performance were modest and not consistent across patients. These results demonstrate that, during reproduction of supra-seconds time intervals, the SMA is involved at the end of the target interval, suggesting a role in the duration categorization and decision making operations or alternatively in the preparedness of the timing of the future movement that will be executed during the reproduction phase.

\section{Keywords}

Duration, electrophysiology, epileptic patients, secs-mins, supplementary motor area (SMA) 


\title{
Dissociable Neural Substrates for Internally-Based and Externally-Cued Timing: A Meta-Analytic Review of Functional Neuroimaging Studies
}

\author{
Alice Teghil1,2,3, Maddalena Boccia ${ }^{1,3}$, Fabrizia D'Antonio ${ }^{2,3,4}$, \\ Antonella Di Vita ${ }^{1,2,3}$, Carlo de Lena ${ }^{4}$ and Cecilia Guarigliaa,3 \\ ${ }^{1}$ Department of Psychology, "La Sapienza" University of Rome, Italy \\ ${ }^{2} \mathrm{PhD}$ Program in Behavioral Neuroscience, "La Sapienza” University of Rome, Italy \\ ${ }^{3}$ IRCCS Fondazione Santa Lucia, Rome, Italy \\ ${ }^{4}$ Department of Neurology and Psychiatry, "La Sapienza” University of Rome, Italy
}

Depending on task and stimulus characteristics, temporal intervals can be processed as absolute durations, or relative to the rhythmic (Teki et al., 2012) or periodic structure of the stimulus itself (Ahrens \& Sahani, 2011). We will refer to the former as internally-based timing (IBT), and to the latter as externally-cued timing (ECT). In this study, we performed a general activation likelihood estimation (ALE) meta-analysis on $177 \mathrm{fMRI}$ experiments from 79 papers to identify the neural underpins of general aspects of timing, and two individual ALEs to test the neural segregation between IBT and ECT. ALE meta-analysis assesses the overlap between activation foci from different experiments by treating them as probability distributions centered at the coordinates of each one (Eickhoff et al., 2009). General ALE revealed activations in bilateral supplementary motor area (SMA), intraparietal sulcus (IPS), inferior frontal gyrus (IFG), insula and caudate nucleus (CN), right posterior superior temporal gyrus (p-STG) and left precentral gyrus (PCG). In individual ALEs, IBT yelded to activation of SMA, IFG and insula, inferior olive and right IPS, whereas ECT recruited bilateral SMA and IFG, right p-STG and CN and left PCG. A conjunction analysis revealed bilateral SMA/pre-SMA, IFG and right CN as commonly involved in IBT and ECT; compared to IBT, ECT yelded to activation in SMA, right IFG, left PCG and insula. In conclusion, we highlighted a broad network in which SMA and CN play a key role, in line with previous results (Wiener et al., 2010), but we also evidenced that both IBT and ECT require the involvement of specific areas, suggesting a possible dissociation between these two processes.

Keywords

MRI, duration, rhythms, adult, 100s of ms-secs

\section{References}

Ahrens, M. B., \& Sahani, M. (2011). Observers exploit stochastic models of sensory change to help judge the passage of time. Curr. Biol., 21, 200-206.

Eickhoff, S. B., Laird, A. R., Grefkes, C., Wang, L. E., Zilles, K., \& Fox, P. T. (2009). Coordinate-based Activation Likelihood Estimation meta-analysis of neuroimaging data: A random-effects approach based on empirical estimates of spatial uncertainty. Hum. Brain Mapp., 30, 2907-2926. 


\title{
Investigating the Different Role of Parietal and Frontal Areas in Temporal Processing Using Non-Invasive Brain Stimulation Techniques: A Study with tRNS
}

\author{
Giovanna Mioni ${ }^{1}$, Diletta Amoroso ${ }^{1}$, Simon Grondin ${ }^{2}$ \\ and Franca Stablum ${ }^{1}$ \\ ${ }^{1}$ Department of General Psychology, University of Padova, Italy \\ ${ }^{2}$ Department of Psychology, Laval University, Québec, Canada
}

\begin{abstract}
Goal of the study: The understanding of the mechanisms underlying the temporal representation of duration in milliseconds/seconds range remains a complex issue. Different brain areas have been identified as critical in temporal processing depending on temporal range and modalities used. We investigate the different role of parietal and frontal areas in temporal processing. Method: Participants performed time bisection tasks, with empty interval marked by visual and auditory stimuli. The task includes a learning phase in which participants were asked to memorize two standard durations: $300 \mathrm{~ms}$ (short standard) and $900 \mathrm{~ms}$ (long standard). After the training phase, participants were required to perform four blocks. During the experimental phase participants performed the task under tRNS stimulation. Forty participants were stimulated over right parietal cortex and 40 participants were stimulated over right frontal areas. Participants were divided into groups depending on the modality of the markers that we used to define the temporal intervals (visual-visual; visual-auditory; auditory-visual and auditory-auditory). Results: Main effect of modality was observed; higher variability was observed in the visual-auditory and auditory-visual condition independently on brain area stimulated. No effect of stimulation in time perception was observed when participants were stimulated over frontal area but higher variability was observed when participants were stimulated over parietal area. Conclusion: The study confirms and extends previous findings indicating the role of parietal cortex in temporal processing of brief intervals and when multi-modalities are used.
\end{abstract}

Keywords

Duration, time bisection task, behavior, brain stimulation, tRNS, adult, 100s of ms-secs

\section{Roles of Cortical and Sub-Cortical Pathways on Time Perception: A Case Study with Agenesis of the Corpus Callosum}

\author{
Yuko Yotsumoto', Miku Okajima1, Akinori Futamura², \\ Motoyasu Honma $^{2}$ and Mitsuru Kawamura ${ }^{2}$
}




\author{
${ }^{1}$ Department of Life Sciences, The University of Tokyo, Tokyo, Japan \\ ${ }^{2}$ Department of Neurology, Showa University School of Medicine, Tokyo, Japan
}

Sometimes, we need to focus on temporal information of a certain event while ignoring the others. The ability or inability to inhibit the task-irrelevant information will offer us insights on the mechanisms of time perception. We recently proposed that modulation of temporal oscillators encoding the interval duration predicts perceived time (Hashimoto \& Yotsumoto, 2015). Based on that model, we hypothesized that temporal information related to visual stimuli are encoded and processed in cortical coordinates, and the interference from task-irrelevant distractors depends on the cortical distance between a to-betimed target item and a distractor. First, we conducted a time reproduction task with or without task-irrelevant distractors placed in various spatial locations. The results showed that when a flickering distractor was located ipsilateral to the target, it caused a greater time dilation than a distractor located contralateral to it. This indicates that a larger cortical distance led to lesser interference from the distractor. Second, we studied the ability to suppress irrelevant intervals by examining a split-brain patient. One patient with agenesis of the corpus callosum and five age- and sex-matched control subjects participated in the reproduction task of the target interval while ignoring the interval of distractors that were displayed in visual field either ipsilateral or contralateral to the target. It was observed that, in the patient, the distractor interferes with the reproduction performance more strongly when it is contralateral than when it is ipsilateral, showing that in the absence of the corpus callosum, the interhemispheric distractor has a larger effect than the intrahemispheric distractor. Our results suggest that visually presented task-relevant items affect time perception in cortical coordinates. The corpus callosum plays a role in the inhibition of interhemispheric interference and in its absence, that temporal interval information can be transferred via subcortical areas.

\title{
Keywords
}

Duration, behaviour, adult, patient, 100s of ms-secs

\section{Reference}

Hashimoto, Y., \& Yotsumoto, Y. (2016) Effect of temporal frequency spectra of flicker on time perception: Behavioral testing and simulations using a striatal beat frequency model. Timing Time Percept., 3, 201-222.

\section{Activity of Cholinergic Interneurons in the Primate Striatum during a Time Estimation Task}

\author{
Anne-Caroline Martel and Paul Apicella
}

Institut de Neurosciences de la Timone, CNRS - Aix Marseille Université UMR7289, Marseille, France 
The striatum is critical for reward-based learning and action selection under control of two closely interacting modulatory systems: midbrain dopamine (DA) neurons and cholinergic interneurons (ChIs) of the striatum. Given the widely held notion that DA is central to timing, one can consider that ChIs may also participate in temporal processing. However, no study has looked at possible links between ChI signaling and the ability to time intervals in the seconds range. To address this issue, we recorded single-neuron activity in the striatum of a monkey performing a time estimation task. At the beginning of each trial, a visual stimulus (timing cue), presented either on the left or the right side, indicated the length of a time interval (1.3 or $2.3 \mathrm{~s}$ ) during which the animal must wait before performing a left or right reaching movement leading to reward. Behaviorally, we verified that the monkey's performance on the task satisfied the scalar property of time estimate. We identified 114 neurons that met established electrophysiological criteria for tonically active neurons (TANs), thought to be ChIs. We observed brief changes in TAN firing associated with the detection of timing cues (39.5\%) which did not vary systematically with interval duration. Since the monkey's accuracy at estimating the interval is lowered in proportion to its duration, we investigated the possibility that activity changes after the cue onset or immediately before the decision to move reflected the timing performance. To do this, performance and neuronal firing were examined on a trial-by-trial basis, separately for short and long intervals. Preliminary results indicate that TANs responses to the cue that signaled the short interval were larger when correct timed responses were initiated, whereas a similar effect was not observed for long interval trials. It therefore appears that local cholinergic innervation of the striatum may integrate temporal information.

\title{
Keywords
}

Duration, prediction, behavior, electrophysiology, monkey, secs-mins

\section{Dorsal Striatum Inactivation Disrupts Temporal Dscrimination, but not Tone Frequency Discrimination in a Bisection Task}

\author{
Taisuke Kamada ${ }^{1}$ and Toshimichi Hata ${ }^{2}$ \\ ${ }^{1}$ Graduate School of Psychology, Doshisha University, Japan \\ ${ }^{2}$ Faculty of Psychology, Doshisha University, Japan
}

Previous studies have demonstrated that lesion or inactivation of the dorsal striatum (DS) disrupts tasks requiring interval timing, time perception in the seconds-to- minutes range (Gouvêa et al., 2015; Meck, 2006). However, it is still unknown whether this effect is specific for interval timing or reflects other deficits (e.g., reward processing). Here, we addressed this question by comparing the role of the DS on a temporal bisection task and a tone frequency bisection task. Rats were presented 1 or $9 \mathrm{kHz}$ tones for 3 or $9 \mathrm{~s}$ (anchor stimuli) and were trained on one of two tasks as follows. During a temporal bisection task, 
the rat's response on lever A was reinforced following a $3 \mathrm{~s}$ tone, while the response on lever B was reinforced following a $9 \mathrm{~s}$ tone. During a tone frequency bisection task, the response on lever A was reinforced following a $1 \mathrm{kHz}$ tone, while the response on lever $\mathrm{B}$ was reinforced following a $9 \mathrm{kHz}$ tone. After the acquisition of discrimination, rats were also presented intermediate frequencies and durations of tones without reinforcement in addition to anchor stimuli to generalize their responses based on the duration or frequency. Therefore, two tasks were completely the same except for the required types of discrimination. Rats were then infused with $\gamma$-aminobutyric acid (GABA) A receptor agonist, muscimol $(0,50$ or $100 \mathrm{ng} / \mu \mathrm{L})$ into the bilateral DS before the sessions. We found that $50 \mathrm{ng}$ muscimol shifted the bisection curve right-ward, while that of $100 \mathrm{ng}$ reduced the response rates to lever $B$ in longer durations in the temporal bisection task. However, the same treatment did not affect the frequency bisection task. These results suggest that the inactivation of the DS disrupts temporal discrimination but not tone frequency discrimination and it plays an important role in interval timing.

\title{
Keywords
}

Duration, behavior, pharmacology, animal, secs-mins

\section{References}

Gouvêa, T. S., Monteiro, T., Motiwala, A., Soares, S., Machens, C., \& Paton, J. J. (2015). Striatal dynamics explain duration judgments. Elife, 4, e11386.

Meck, W. H. (2006). Neuroanatomical localization of an internal clock: A functional link between mesolimbic, nigrostriatal, and mesocortical dopaminergic systems. Brain Res., 1109, 93-107.

\section{Role of Temporal Prediction Error on Triggering Plasticity in Amygdala Nuclei in an Appetitive Conditioning}

\author{
Tatiane Ferreira Tavares ${ }^{1}$, José Lino Oliveira Bueno ${ }^{1}$ and Valérie Doyère ${ }^{2}$ \\ ${ }^{1}$ University of Sao Paulo, Faculty of Philosophy, Sciences, and Letters of Ribeirao Preto, \\ Department of Psychology, Section of Psychobiology, Ribeirao Preto, Brazil \\ ${ }^{2}$ Paris-Saclay Institute of Neuroscience (Neuro-PSI), CNRS-UMR9197, Paris-Sud, Orsay, \\ France
}

The important role of time in associative learning was incorporated in temporal difference learning models and is the foundation of the prediction error detection studies. Studies have shown that the amygdala is involved in these processes, and that prediction error detection is a fundamental mechanism in triggering reconsolidation of Pavlovian memories in the amygdala (Díaz-Mataix et al., 2014). This study used the Reinforcement Omission Effect (ROE) paradigm (Tavares et al., 2014) to analyze how an unexpected change 
in the temporal parameters of the association (error detection) interferes with memoryupdating in an appetitive operant paradigm (Experiment 1). Experiment 2 then assessed whether infusion of a protein synthesis inhibitor (anisomycin) in basolateral amygdala (BLA) immediately before Error session interferes with the temporal error detection. In Experiment 1, rats were trained to FI 12 s LH 6 s or FI 6s LH 12 s under 100\% of Reinforcement. They were then submitted to one session of Error with changes or no changes in the duration of FI LH schedule (100\% of Reinforcement). Then, they were exposed to ROE test, which consisted of the same schedule as Training, but with only $50 \%$ of trials reinforced. In Experiment 2, rats were submitted to the same phases, except that they received infusion of anisomycin in the BLA immediately before the Error session. The results showed that the temporal change in the Error session of Experiment 1 interfered in the ROE, affecting processes related to learning and memory-updating. In addition, in the Experiment 2, the infusion of anisomycin interfered in these processes, suggesting memory-updating interferences.

Keywords

Prediction error, behavior, neuropsychology, amygdala, rats, secs-mins

\title{
References
}

Díaz-Mataix, L., Tallot, L., \& Doyère, V. (2014). The amygdala: A potential player in timing CS-US intervals. Behav. Processes, 101, 112-122.

Tavares, T. F. T, Judice-Daher, D. M., \& Bueno, J. L. O. (2014). Large neurotoxic amygdala lesion impairs reinforcement omission effects. Behav. Brain Res., 266, 1-6.

\section{Medial Prefrontal Cortex Activity of Rats in the Differential Reinforcement Response Duration Task}

\author{
Gabriela C. Tunes, Eliezyer F. de Oliveira, Marcelo S. Caetano \\ and Marcelo B. Reyes \\ CMCC/UFABC, São Bernardo do Campo, Brazil
}

\begin{abstract}
Although time is a fundamental dimension of life, we don't know yet how and where it is encoded in the brain. Tasks such as temporal bisection or fixed interval have been used to investigate neural correlates of time perception. However, in electrophysiological studies it is usually difficult to disentangle the motor activity from the time perception correlates. The Differential Reinforcement Response Duration (DRRD) task can be a good alternative because the animal has to hold a response during a criterion interval in order to receive a reward, and therefore the motion of the animal is reduced during the trial. In our work, we recorded neurons from the medial prefrontal cortex of rats while they perform the DRRD task with $1500 \mathrm{~ms}$ criteria. By analyzing the firing rate while the animal
\end{abstract}


holds the response, we were able to identify patterns of neural activity correlated with time perception, as for example ramping neurons already described in classical temporal estimation tasks.

\title{
Keywords
}

Duration, prediction, behavior, electrophysiology, wistar

\section{Study of the Duration Discrimination Task and the Shift of Bisection Point in Rats}

\author{
Shogo Sakata ${ }^{1}$ and Yuta Nakamura ${ }^{2}$ \\ ${ }^{1}$ Department of Behavioral Sciences, Hiroshima University, Japan \\ ${ }^{2}$ Graduate School of Integrated Arts \& Sciences, Hiroshima University, \\ Higashi-Hiroshima, Japan
}

Timing is very important to survival in all animals. It is known that animals have relatively accurate timing ability of intervals. However, neural mechanisms of time perception are still unknown. The purpose of this study is to investigate the brain function with electrical activity on temporal discrimination behavior. Six Wistar-Imamichi rats were trained using temporal discrimination task and tested by interval bisection task with non-reinforcement probe trials. In the experiment, rats discriminated between stimulus duration of 2 sec versus $8 \mathrm{sec}$ with $4 \mathrm{~Hz}$ pulse tone stimulus. They were trained for 20 sessions with temporal discrimination task in two lever operant chambers with food reinforcement. After training of 2 sec versus 8 sec duration discrimination task, they acquired over $80 \%$ correct responses. In the interval bisection task with 2.5, 3.2, 4.0, 5.0 and $6.4 \mathrm{~s}$ intermediate empty trials, they showed that the bisection point shifted for rightward compared with continual tone stimulation. After this probe test, we retrained for temporal discrimination task between $2 \mathrm{~s}$ versus $8 \mathrm{~s}$ with continual tone stimulation for 10 sessions. There were any more no differences at bisection point in the interval bisection task with continual tone and pulse tone. Then we implanted electrodes and measured EEG from five regions. The auditory evoked potentials showed the clear N1P2 potentials. These results suggest that first original stimulus property with temporal discrimination training affects time perception especially for temporal evaluation with pulse stimulus, not with continual stimulus.

\section{Keywords}

Timing, duration discrimination, temporal bisection task, evoked potentials, rat 


\title{
Development of Schedule-Induced Drinking in the Bi-Peak Procedure
}

\author{
Gabriela E. López-Tolsa and Ricardo Pellón \\ Universidad Nacional de Educación a Distancia, Spain
}

\begin{abstract}
Organisms exposed to intermittent reinforcement scheduled display behaviours that consistently occur in the inter-reinforcement times (IRT), even when no contingency has been arranged between those behaviours and the reinforcer. Those behaviours are called adjunctive behaviours. The most studied example of adjunctive behaviours is scheduleinduced drinking (SID), which consists on an excessive drinking during the IRT in food reinforcement schedules. It has been proposed that adjunctive behaviours serve time estimation. Also, that adjunctive behaviours are part of a behavioural pattern that occurs in every IRT, shaped by the environmental conditions including time regularities (Ruiz et al., 2016). The shape of those behavioural patterns can aid organisms to have better performances in certain temporal learning tasks. The aim of this study was to observe the performance on a bi-peak procedure when rats develop SID. In the training phase rats would receive a reinforcer after pressing a lever associated with a 20 -sec fixed interval (short trial) or another lever associated with an 80-sec fixed interval (long trial). Trials were randomly alternated with no external cue that indicated the value of the schedule. In the testing phase rats were exposed to short and long trials and to peak trials that lasted for 150-sec after which no reinforcer was delivered. Only half of the subjects had access to water in the experimental chamber. Results showed that rats with access to water developed a behavioural pattern that consisted on drinking, pressing the short lever and pressing the long lever; whereas rats without access to water started pressing the short lever earlier in the trial, and then switched to the long lever. These results are consistent with previous studies that show that SID occurs in the beginning of the trial (Pellón \& Killeen, 2013) and that behavioural patterns are repeated once every trial (Ruiz et al., 2016).
\end{abstract}

\section{Keywords}

Duration, behaviour, rats, secs-mins, adjunctive behaviours

\section{References}

Killeen, P. R. \& Pellón, R. (2013). Adjunctive behaviors are operants. Learn. Behav., 41, $1-24$.

Ruíz, J. A., López-Tolsa, G. E. \& Pellón, R. (2016). Reinforcing and timing properties of water in the schedule-induced drinking situation. Behav. Processes, 127, 86-96. 


\title{
Proprioceptive Timing Mediates Switch Behavior in CD-1 Mice
}

\author{
Anna Roselle and J. Mark Cleaveland \\ Vassar College, USA
}

The consolidation of behavioral sequences into relatively ballistic habits is thought to involve the formation of stimulus - response (S-R) associations (Balleine \& O'Dougherty, 2010; Graybiel, 1998). Typically, the stimuli in these associations are assumed to be exterioceptive, i.e., external to the organism. A red traffic light, for example, might occasion the response "press brake." However, responses, themselves, also possess stimulus properties that can mediate behavior (e.g., Cleaveland, Jäger, Rößner, \& Delius, 2003). Indeed, these "proprioceptive cues" have long been hypothesized to underlie habit formation (Hull, 1934). One such stimulus involves the time durations between responses - a stimulus termed interresponse time (IRT). We hypothesize that IRTs can come to serve as stimuli that differentially control response elements during habit formation. To examine this hypothesis we report on two experiments that asked whether CD-1 mice utilize IRTs to structure behavior in a two-choice environment. In experiment 1 , eight mice were exposed to a free-operant concurrent variable-interval (VI) 20-s VI 60-s reinforcement schedule. We found that switch and stay responses were differentially correlated with IRT durations. Further, Monte Carlo simulations utilizing IRT-switch functions provided a close fit of our subjects' actual response structures. In Experiment 2 we directly and differentially reinforced stay / switch responses based on IRT durations in a two-lever procedure. For four of the subjects, the probability of receiving reinforcement after switch responses was proportional to IRT duration. For five of the subjects, these reinforcement probabilities were inversely proportional to IRT duration. Regardless, all of our subjects learned to emit IRT mediated switching behavior that matched the reinforcement contingencies. Both experiments 1 and 2 showed that IRTs come to control sequential choice behavior in CD-1 mice. We conclude our poster, therefore, by briefly considering the role that IRT-behavior associations might play in the development of stereotypies.

\section{Keywords}

Mice, interresponse time, habit consolidation

\section{Embodied Learning of Time Intervals}

\author{
Elizabeth Thomas $^{1}$, Robert French ${ }^{2}$, Lilian Fautrelle ${ }^{3}$ and Jennifer Coull ${ }^{4}$ \\ IINSERMU1093, UFR STAPS, Université de Bourgogne, France \\ ${ }^{2}$ CNRS UMR5022, LEAD, Université de Bourgogne, France \\ ${ }^{3}$ UFR STAPS, Université Paris Ouest, Nanterre, France \\ ${ }^{4}$ CNRS, LNC Aix Marseille Université, France
}


Previous studies have shown that temporal predictability leads not only to faster reaction times (RTs) but also to alterations in non-temporal aspects of the reaction such as response force (Mattes \& Ulrich, 1997). This implies that that the electrical activities generating muscle forces are modified as a function of temporal expectation. Analyzing muscular electrical activity (EMGs) provides a window on how the neural commands for responses are modified with improvements in temporal prediction. In this study we examined how EMGs of two ankle antagonist muscles, the soleus and tibialis, are modified as subjects learn the temporal regularity of a sequence. The study was carried out with two groups - one which responded to the regular appearance of a visual stimulus 50 times, every $3 \mathrm{~s}$. (R group) and a second which responded to the irregular presentation of the same stimulus (IR group) with intervals ranging from 2 to $4 \mathrm{~s}$. Subjects responded to the appearance of the stimulus by bending over to press a button that was slightly out of reach. This whole-body response was chosen because trunk forward flexion requires an anticipatory reduction in the electrical activity in the soleus muscle even before the onset of visible movement. The two groups showed significant differences in their response characteristics as a function of stimulus- presentation type. Participants in the IR group showed typical foreperiod and sequential effects, with RTs decreasing as a function of interval duration. Participants in the R group showed greater decreases in RT over the session than those in the IR group, indicating learning of the regular temporal interval. Participants in the R group showed parallel changes in soleus and tibialis EMG activity over the session, corresponding to improved response anticipation and reduced force, respectively. These data indicate that temporal learning is accompanied, and possibly even enhanced, by dynamic changes in muscular activity.

\title{
Keywords
}

Prediction, behavior, EMG, adult, secs-mins

\section{Reference}

Mattes, S., \& Ulrich, R. (1997). Response force is sensitive to the temporal uncertainty of response stimuli. Percept. Psychophys., 59, 1089-1097.

\section{Embodied or "Embrained" Temporal Precision?}

\author{
Leon van Noorden \\ IPEM, Ghent University, Belgium
}

Basic rhythmic movements have a tendency to be concentrated around the frequency of $2 \mathrm{~Hz}$ (e.g., walking of adults and arm movements of babies; Provasi \& Bobin-Bègue, 2003; Stijns et al., 2007). It appears also in the distribution of tempi of musical pieces and in a number of rhythmical phenomena (Van Noorden \& Moelants, 1999). The results of an experiment about the synchronisation skill of children between 3 and 11 years old may 
indicate that both an embodied - emergent - and an "embrained" - event related - timing mechanism play a role in their development (Van Noorden et al., 2017).

\title{
References
}

Provasi, J., \& Bobin-Bègue, A. (2003). Spontaneous motor tempo and rhythmical synchronization in 2 1/2- and 4-year-old children. Int.J. Behav. Dev., 27, 220-231.

Stijns, F., van Noorden, L., Moelants, D., \& Leman, M. (2007). Walking on music. Hum. Mov. Sci., 26, 769-785.

Van Noorden, L., De Bruyn, L., van Noorden, R. \& Leman, M. (2017). Embodied social synchronization in children's musical development. In M. Lesaffre, P.-J. Maes \& M. Leman (Eds.), The Routledge companion to embodied music interaction. New York: Routledge.

Van Noorden, L., \& Moelants, D. (1999). Resonance in the perception of musical pulse. Journal of New Music Research, 28, 43-66.

\section{Intention and Prediction in Time Perception}

\section{Mukesh Makwana and Narayanan Srinivasan}

\author{
Centre of Behavioural and Cognitive Sciences, University of Allahabad, Allahabad, India
}

Human beings are intentional agents with predictive capabilities. Recently pre- activation account has been used to explain temporal expansion (Press et al., 2014). As both intention and prediction involves pre-activation of upcoming events, it is crucial to understand their role in modulating time perception. In present study, we orthogonally manipulated intention and prediction in temporal bisection task using a method of constant stimuli. Participants were trained for two anchor durations (short-300 ms; long-700 ms). In main experiment, before each trial, participants indicated what color circle they intend to see (red/green), by pressing a predefined key. After $250 \mathrm{~ms}$ of their keypress, either intended or unintended color target circle appeared on the screen. The duration of target circle was varied randomly among nine levels (300 ms-to-700 ms in steps of $50 \mathrm{~ms}$ ). Participants reported the perceived duration of the test circle as closer to the short or long anchor duration. Half of the participants got the intended color circle with highprobability $(0.75)$ and unintended color with low probability $(0.25)$, whereas the other half got the unintended color circle with high probability (0.75) and intended color with low probability $(0.25)$. Point of subjective equality (PSE) and difference limen (DL) were calculated for each condition. Results showed a significant interaction between intention and prediction. Surprisingly when the highly predicted outcome also happened to be the intended one, it was perceived as longer in duration compared to unintended condition. In literature, typically the unpredicted outcome is perceived as longer compared to predicted one (Ulrich et al., 2004), as low probable outcome grabs more attention and high probable outcome shows repetition suppression (Pariyadath \& Eagleman, 2007). However, in our study intention is reversing the general effect of prediction on duration perception. Further studies are needed to validate these findings and understand how these two processes interact at neural level. 


\title{
Keywords
}

Duration, behavior, adult, 100ms-sec, intention, prediction

\section{References}

Pariyadath, V., \& Eagleman, D. (2007). The effect of predictability on subjective duration. PLoS One, 2, e1264.

Press, C., Berlot, E., Bird, G., Ivry, R., \& Cook, R. (2014). Moving time: The influence of action on duration perception.J. Exp. Psychol. Gen., 143, 1787.

Ulrich, R., Nitschke, J., \& Rammsayer, T. (2006). Perceived duration of expected and unexpected stimuli. Psychol. Res., 70, 77-87.

\section{Temporal and Spatial Attention in Touch}

\author{
Alexander Jones \\ Middlesex University London, UK
}

Selective spatial attention distinguishes between endogenous (top down) and exogenous (bottom-up) orienting. That is, attention can voluntarily be directed to a location, e.g., the left hand, or attracted to a location by an external event, e.g., a fly landing on the hand. However, selection relies not only on where but also when an event occurs. Temporal attention can also be defined in terms of endogenous orienting whereby we intentionally anticipate when an event will occur, but also, temporal attention can be exogenous and generated by an external temporal pattern. Temporal and spatial attention are two mechanisms which have independently shown to affect stimulus processing but it remains largely unknown how, and if, they interact. In a series of experiments, measuring response times (RTs) and EEG, the work presented here investigates how exogenous temporal attention, in the form of rhythmic cueing, interacts with endogenous and exogenous spatial attention in touch. In an endogenous task, participants received informative bilateral rhythmic stimuli to the fingers prompting them to attend to the left or right. Tactile targets were unilateral and appeared at the attended $(80 \%)$ or unattended hand $(20 \%)$. Additionally, and irrelevant to the task, targets appeared in or out of synchrony with the rhythmic cue. The results show effects of both endogenous spatial attention (facilitation of attended targets) and rhythmic cueing (hazard function) but no evidence that they interacted. In the exogenous task, cues where rhythmic but unilateral and non-informative, regarding the upcoming target location. Here we observe inhibition of return with overall slower RTs for cued compared to uncued targets. Importantly, there was an interaction between exogenous spatial and exogenous temporal attention. These findings suggests endogenous spatial and exogenous temporal attention are dissociable processes whilst exogenous temporal and exogenous spatial attention interact in the sense of touch. 


\title{
Paying Attention to Time Is Faster than Paying Attention to Space
}

\author{
Yaffa Yeshurun and Shira Tkacz-Domb \\ Psychology Department, University of Haifa, Israel
}

\begin{abstract}
It is well known that we can voluntarily allocate attention to a specific point in time at which we expect a relevant event to occur. Here, we employed the constant foreperiod and temporal orienting paradigms to examine the time course of this endogenous temporal attention. With both paradigms, the task was unspeeded identification of a letter presented for a brief duration (16 ms), preceded by a warning signal. The warning signal was either auditory or visual, and it was either informative - indicating the most likely foreperiod (the interval between the warning signal and the target), or non-informative. Critically, to avoid effects of exogenous temporal attention both types of warning signal did not include an intensity change. Additionally, unlike previous studies, we included a wide range of foreperiods (25-2400 ms). In comparison to a non-informative warning signal, identification accuracy was significantly higher when the warning signal indicated the most likely foreperiod. Importantly, such effects of temporal attention were found with both very long and very short foreperiods, suggesting optimal voluntary allocation of attention within $150 \mathrm{~ms}$ and up to $2.5 \mathrm{sec}$. Given that letter identification was not speeded we can conclude that voluntary allocation of temporal attention to a specific point in time improves perceptual processing. Moreover, such allocation of endogenous temporal attention is extremely fast - twice as fast as endogenous spatial attention.
\end{abstract}

\section{Keywords}

Prediction, attention, behavior, adult, 10s-100s of ms, 100s of ms-secs

\section{Electrophysiological Correlates of Spatial Attention and Rhythms in Sensory Processing}

\author{
Louise C. Barne', Floris P. de Lange ${ }^{2}$ and André M. Cravo ${ }^{1}$ \\ ${ }^{1}$ Centro de Matemática, Computação e Cognição, Universidade Federal do ABC (UFABC), \\ São Bernardo do Campo, Brazil \\ ${ }^{2}$ Centre for Cognitive Neuroimaging at the Donders Institute for Brain, Cognition and \\ Behaviour, Radboud University, Nijmegen, The Netherlands
}

Attention is a fundamental process to optimize the computational capacity of a biological system. It has been previously suggested that entrainment can work as a core mechanism 
of selective attention (Lakatos et al., 2008). In the present study, we investigated the role of low-frequency neural oscillations when human participants had to attend to a rhythmic stream $(1 \mathrm{~Hz})$ in one location of their visual field while ignoring another stream presented in antiphase to the rhythm. The experimental protocol was approved by the Ethics Committee on Human Research of UFABC. Nineteen volunteers had to identify visual oddball stimuli within a stream of standard stimuli presented only at attended side. During the experimental procedure, electrophysiological recordings was acquired continuously from 64 electrodes positioned according to the 10-10 International system. For the time-frequency analysis, power was estimated using a single Hanning taper between 4 to $40 \mathrm{~Hz}$. For frequencies close to stimulus presentation rate, data was low-pass filtered and phase values were computed using a Hilbert transform. We found stronger alpha desynchronization (9-15 Hz) in anticipation to stimulus presentation in attended compared to unattended streams $[$ cluster-stat $=502.33, \mathrm{p}=0.03$ ]. Moreover, the phase of low-frequency oscillations was concentrated in opposite angles in attended versus unattended streams $[\mathrm{F}(2,17)=$ $4.34, \mathrm{p}=0.03$ ]. Our results suggest that neural oscillations are correlated with sensory processing of attended and ignored stimuli.

\title{
Keywords
}

Rhythms, attention, electrophysiology, adult, 100s of ms-secs

\section{Reference}

Lakatos, P., Karmos, G., Mehta, A. D., Ulbert, I., \& Schroeder, C. E. (2008). Entrainment of neuronal oscillations as a mechanism of attentional selection. Science, 320, 110-113.

\section{Can Multivariate Pattern Analysis Help Us Understand How Time Is Encoded?}

\author{
André Mascioli Cravo \\ Center for Mathematics, Computing and Cognition, Federal University of \\ ABC (UFABC), Brazil
}

The ability to process time on the scale of milliseconds and seconds is essential for behavior. A growing number of studies have started to focus on brain dynamics as a mechanism for temporal encoding. Although there is evidence in favor of this view from computational and in vitro studies, there is still a lack of results from experiments in humans. In this poster, I will discuss recent EEG studies in which we use multivariate pattern analysis to study temporal processing. Using different temporal tasks, we show that these techniques can capture basic properties of temporal perception. Importantly, they can also help elucidate key questions about the mechanisms of temporal encoding, for example whether intervals marked by different sensory modalities share a common neural representation. We show that these techniques can bring important advances in our understanding of temporal processing. 


\title{
The Influence of Temporal Predictability on Response Inhibition
}

\author{
Inga Korolczuk ${ }^{1}$, Boris Burle ${ }^{2}$ and Jennifer T. Coull ${ }^{2}$ \\ Jagiellonian University, Krakow, Poland \\ ${ }^{2}$ Aix-Marseille University \& CNRS, Marseille, France
}

We continuously adapt our behavior to a changing environment. Such adaptation not only requires the selection of appropriate responses but also prevention of unwanted ones. Moreover, these responses (or lack thereof) must occur at appropriate moments in time. "Temporal orienting" is the process by which we direct attentional resources to selected moments in time. Most studies on temporal orienting have investigated responses based on relatively simple, noncompeting stimulus-action representations (though see Correa et al., 2010). Here, we examined how response inhibition is affected by temporal orienting by including informative cues that directed temporal attention on a trial-by-trial basis (Coull \& Nobre, 1998) in inhibitory control tasks. We compared performance on classic response inhibition paradigms (Simon task and stop-signal task) when cues either predicted (temporal cues) or not (neutral cues) when the subsequent target would appear. In the Simon task, as expected, reaction times (RTs) were faster when the target appeared in a location congruent with its required response. This congruency effect was exacerbated in the temporal versus neutral cue condition i.e. when targets were temporally predictable. Moreover, analyzing the probability of making an error as a function of response speed ("Conditional Accuracy Function") indicated that temporal cueing increased the likelihood of fast, impulsive, errors. Analysis of the stop-signal paradigm revealed that temporal predictability impaired response inhibition. The Stop-Signal Delay (SSD) was shorter in temporal versus neutral cue trials and Stop Signal Response Time (SSRT), a covert measure of the stopping process in response inhibition, was longer. These data indicate that participants needed a longer time to successfully inhibit responses to a target if they had already formed a temporal expectation of when the target would occur. Taken together, our results suggest that temporal predictability exacerbates the impulse to execute a prepotent response.

\section{Keywords}

Duration, prediction, behavior, adult, 100s of ms-secs

\section{References}

Correa, Á., Cappucci, P., Nobre, A. C., \& Lupiáñez, J. (2010). The two sides of temporal orienting: Facilitating perceptual selection, disrupting response selection. Exp. Psychol., $57,142-148$.

Coull, J. T., \& Nobre, A. C. (1998). Where and when to pay attention: The neural systems for directing attention to spatial locations and to time intervals as revealed by both PET and fMRI.J. Neurosci., 18, 7426-7435. 


\section{Disentangling between Voluntary and Automatic Temporal Orienting: A High Spatial-Resolution ERP Study}

\section{Giovanni Mento}

Department of General Psychology, University of Padova, Italy

A main distinction has been proposed between voluntary and automatic mechanisms underlying temporal orienting of attention (TO). Voluntary TO implies the endogenous directing of attention induced by symbolic cues. Whereas, automatic TO is exogenously and effortlessly instantiated by the physical properties of stimuli by themselves. A well-known example of automatic TO are the sequential effects (SEs), which refer to the adjustments of participants' behavioural performance as a function of the trial-by-trial sequential distribution of the foreperiod (FP) between two stimuli. While the neural networks underlying voluntary TO are relatively understood, the neural bases of automatic SEs have been much less investigated. In this study a group $(n=16)$ of healthy adults underwent a temporal cueing detection task recently employed to assess the behavioural advantage induced by both the cue and the sequence of a variable FP (Mento \& Tarantino, 2015; Mento \& Vallesi, 2016). Moreover, during the task the high-spatial resolution electrophysiological activity (128 sensors) was recorded with the purpose of disentangling between the spatiotemporal neural signatures underpinning voluntary and automatic TO. The results identified in the cue-locked central P3a and in the posterior P3b two distinct ERP markers reflecting automatic and voluntary expectancy generation, respectively. The brain source reconstruction further revealed that distinct cortical circuits supported these two components. Namely, a sensorimotor network (i.e., bilateral precuneus and left intra parietal sulcus) and a more distributed frontoparietal network (i.e., bilateral precuneus, right parietal and bilateral prefrontal areas) were identified as generators of the P3b and the P3a, respectively. Additionally, the findings indicated in the subsequent CNV a common hallmark of both voluntary and automatic expectancy implementation, although partially dissociable neural networks subserved these two mechanisms. Overall, these results provide additional electrophysiological confirmation of previous neuroimaging evidence suggesting that distinct neural substrates can be recruited depending on the cognitive nature of TO.

\section{Keywords}

Prediction, electrophysiology, adult, 100s of ms-secs

\section{References}

Mento, G., \& Tarantino, V. (2015). Developmental trajectories of internally and externally driven temporal prediction. PloS One, 10, e0135098.

Mento, G., \& Vallesi, A. (2016). Spatiotemporally dissociable neural signatures for generating and updating expectation over time in children: A high density-ERP study. Dev. Cogn. Neurosci., 19, 98-106. 


\title{
Time, Prediction and Minimal Self in Schizophrenia
}

\author{
Brice Martin ${ }^{1}$, Jennifer Coull ${ }^{2}$, Michel Cermolacce ${ }^{3}$, Nicolas Franck ${ }^{1}$ \\ and Anne Giersch ${ }^{4}$ \\ ${ }^{1}$ CL3R- SUR, Hôpital du Vinatier, Lyon. Université Lyon 1 \& UMR 5229, France \\ ${ }^{2}$ UMR 7291, Aix-Marseille Université, France \\ ${ }^{3}$ Service Universitaire de Psychiatrie. Hôpital Ste Marguerite, Marseille, France \\ ${ }^{4}$ INSERM U1114, Strasbourg, France
}

Temporality disorders in schizophrenia have been the subject of numerous clinical descriptions, particularly from the psychiatric phenomenology. The latter considers time as an essential component of subjective experience. Husserl describes an automatic integration of the past, the present, and the future. For example, when we listen to a melody, we are conscious of the present note but still have the previous note in mind ("retention") and usually anticipate the note to come. These automatic processes would help the subject to experience his own mental life as stable and continuous. One of the phenomenological models of schizophrenia disorders postulates a breakdown of the temporal structure of consciousness, which consequently induces disturbances of the subjective experience at its most basic level (the level of minimal self) and determines an experience of strangeness (Fuchs, 2007). However, it is difficult to describe the temporal structure of the patients' experience by means of an introspective method. Exploring temporality with cognitive sciences could help to overcome these limitations. Recent work focusing on the alteration of simultaneity judgments and basic temporal predictive processes provides an encouraging set of data on the way to a cognitive understanding of the patients' experience of time (Lalanne et al., 2012). We'll present recent work exploring both overt and implicit measures of temporal prediction with a dedicated paradigm (in this case, the temporal orienting task) and the links between time prediction and minimal self by looking for correlations between performances on temporal tasks and phenomenologically inspired measures of the minimal self. The main result of this work is the correlation between minimal self disorders scores and temporal performance indicating that the more patients show disturbances in the minimal self, the more implicit temporal prediction is altered. We discuss in conclusion the implications for diagnosis, research (research of neuro physiological correlates) and therapeutics (cognitive remediation).

\section{Keywords}

Prediction, behavior, adult, schizophrenia, minimal self

\section{References}

Fuchs, T. (2007). The temporal structure of intentionality and its disturbance in schizophrenia. Psychopathology, 40, 229-235.

Lalanne, L., Van Assche, M., Wang, W., \& Giersch, A. (2012a). Looking forward: An impaired ability in patients with schizophrenia? Neuropsychologia, 50, 2736-2744. 


\title{
Cortical Network for Internal Representation of a Rhythmic Stimulus
}

\author{
Eduardo Rojas-Hortelano and Víctor de Lafuente \\ Neurobiology Institute, UNAM, Mexico
}

Experiments with humans and non-human primates have related a motor corticothalamic- basal ganglia circuit with the production of rhythmic movements (Bengtsson et al., 2005; Merchant et al., 2015). These experiments use a synchronization-continuation task (SCT) that needs a rhythmic finger-tapping movement to evaluate the performance of the subjects (Zarco et al., 2009). A key remaining question is if this network is still involved when the rhythmic stimulus is internally represented (i.e., without movement). To evaluate this, we designed a variation of the SCT that does not need any movements to be performed. 15 volunteers performed our SCT during an fMRI scanning session. During the synchronization phase, the participants watched a periodic stimulus that appeared and disappeared in three different fixed positions at regular intervals. The changes in position created an overall perception of a clockwise or counterclockwise rotation of the stimulus. After three intervals the continuation phase started, in which the stimulus stopped appearing and the subjects had to keep imagining the changes in position as a function of time. After randomly selecting five or six intervals, a visual cue instructed the subjects to indicate the position that they estimated. All subjects also performed a working memory task that we used as control because it had the same sensory components. To assess the areas involved in our SCT version we compared it with the control task using the general lineal model approach. This analysis revealed that the same timing circuit involved in motor timing task is present in our non-motor SCT. We also compared the synchronization and continuation phases. This analysis shows that during the synchronization phase visual and parietal areas are recruited, but during the continuation phase only the premotor cortices survived the analysis suggesting that their relevance in timing tasks goes beyond motor control.

\section{Keywords}

fMRI, cortical circuit, internal representation

\section{References}

Bengtsson, S. L., Ehrsson, H. H., Frossbert, H., \& Ullen, F. (2005). Effector-independent voluntary timing: Behavioural and neuroimaging evidence. Eur. J. Neurosci., 22, 3255-3265.

Merchant, H., Pérez, O., Bartolo R., Méndez, J. C., Mendoza, G., Gámez, J., Yc, K., \& Prado, L. (2015). Sensorimotor neural dynamics during isochronous tapping in the medial premotor cortex of the macaque. Eur.J. Neurosci., 41, 586-602.

Zarco, W., Merchant, H., Prado, L., \& Méndez, J. C. (2009). Subsecond timing in primates: Comparison of interval production between human subjects and rhesus monkeys. J. Neurophys., 102, 3191-3202. 


\title{
Motor Synchronization in Schizophrenia: Preserved Rhythmic Representation with Abnormalities in Predictive Timing
}

\author{
Hélène Wilquin ${ }^{1}$, Yvonne Delevoye-Turrell ${ }^{2}$ and Anne Giersch ${ }^{3}$ \\ ${ }^{1}$ CE1Aix-Marseille Université, LPCLS EA 3278, Aix en Provence, France \\ ${ }^{2}$ University of Lille, CNRS9193, SCALab, Villeneuve d'Ascq, France \\ 3INSERM U1114, Strasbourg, France
}

\begin{abstract}
Disorganization symptoms in schizophrenia may be related to abnormal cognitive representations for the planning and execution of structured motor behavior. But schizophrenia is also associated to motor timing deficits. In the present study, we designed a spatial sensorimotor synchronization task in order to test whether motor fluency is related to deficits in rhythmic pattern organization and/or impaired abilities to synchronize selfinitiated movements with a metronome. 24 chronic schizophrenia patients (SZ) matched with 27 controls (HC) produced sequential-tapping motor actions in synchrony with a regular metronome. These rhythmic sequences were performed towards 6 visual targets that were arranged in the form of a hexagon and presented on a tactile screen. Ten trials of isochronous and non-isochronous sequences respectively constituted of equivalent $(500 \mathrm{~ms})$ and alternated time intervals $(300 / 600 \mathrm{~ms})$ were performed. Patients with schizophrenia were able to produce the tapping rate of isochronous auditory sequence as accurately as controls producing inter response intervals (IRI) close to the expected interval (502 ms). Nevertheless, mean asynchrony errors and variances were larger in patients than in controls for both types of auditory sequences. The patterns of results suggest that patients with schizophrenia are able to act upon an internal representation of a rhythmic pattern but are impaired in the ability to initiate self-selected actions in time to synchronize with external events.
\end{abstract}

\section{Separating Spatial and Interval-Timing Strategies on a Rotating Arena: Animal Model of Schizophrenia}

\author{
Kristyna Maleninska ${ }^{1,2}$, Pavla Jandourkova ${ }^{2,4}$, Ales Stuchlik ${ }^{2}$ \\ and Tereza Nekovarova ${ }^{1,3,5}$ \\ ${ }^{1}$ National Institute of Mental Health, Klecany, Czech Republic \\ ${ }^{2}$ Department of Neurophysiology of Memory, Institute of Physiology Academy of \\ Sciences of the Czech Republic v.v.i., Prague, Czech Republic \\ ${ }^{3}$ Department of Zoology, Faculty of Natural Sciences, Charles University, Prague, Czech \\ Republic
}


${ }^{4}$ Department of Physiology, Faculty of Natural Sciences, Charles University, Prague, Czech Republic

${ }^{5}$ Charles University in Prague, Third Faculty of Medicine, Czech Republic

During navigation both spatial and temporal cues are perceived; every movement in space thus becomes inherently also a movement in time. To study separately temporal and spatial strategies in navigation we modified active place avoidance task in carousel arena for rats. The objective of presented study was to test a rat model of schizophrenia induced by dizocilpine (MK-801) in a variant of active place avoidance task aimed at both spatialbased and temporal-based avoidance strategies. We used a modified active place avoidance task on a rotating arena for rats. Adult male Long-Evans rats were used. This task required the rats to localize a to-be-avoided sector defined in the stable room coordinate frame while the arena rotated. In standard conditions, the rats use allothetic spatial cues to orient and solve the task. However, we introduced time intervals when light was off and thus using of distal cues was disallowed. We assume, that rats had to rely on temporal updating of the sector position in relation to arena rotation. After pretraining in 20-min daily session during four weeks, we have applied MK-801 (0.12 and $0.15 \mathrm{mg} / \mathrm{kg})$ and recorded changes in spatial and temporal performances. The preliminary results corroborated the hypothesis that both types of strategies are separable. Notably, neural circuits affected in patients with schizophrenia are those important also for interval timing and temporal strategies. As disruption of time cognition is often observed in schizophrenia patients, our task can serve as a useful tool to provide insight into deficits of information processing in models of schizophrenia.

\title{
Keywords
}

Duration, rat, behaviour, secs-mins

\section{Acknowledgements}

This study was funded by the project Nr. LO1611 with a financial support from the MEYS under the NPU I program; by Progres Q 35 and by grants GACR 17-04047S, AZV 15-34524A, 17-30833A. Institutional support for IPHYS was provided also by RVO: 67985823.

\section{The Timing Mechanism in the Hundreds of Milliseconds Is Disrupted by a D2 Agonist}

\author{
Karyna Yc, Luis Prado and Hugo Merchant \\ Instituto de Neurobiología, Universidad Autónoma de México (UNAM), \\ campus Querétaro, México
}

Sensory-motor entrainment refers to the ability to synchronize motor actions with a sensory metronome. This ability, crucial for complex behaviors such as music and dancing, depends on the accurate estimation of time. Studies in rodents have shown that the 
dopaminergic system is involved in the perception and production of time intervals in the range of seconds (Meck, 1996). Coincidently, it is well known that the estimation of time in Parkinsonian patients is impaired (Merchant, 2008). Here, we address the functional role of family receptors D2 in the rhythmic production of intervals in the range of the hundreds of milliseconds. We analyzed the effect of systemic administration of the D2 agonist quinpirole on the performance of two Rhesus monkeys in a Synchronization Task (ST) and a serial reaction time, Control Task (CT). In each trial of the ST, the monkeys synchronized their tapping with a visual sensory metronome to produce five isochronous intervals. Six target intervals from 450 to $950 \mathrm{~ms}$ were employed. We measured the variability and accuracy of the produced intervals, as well as the stimulus-response asynchronies. We found that the highest quinpirole dosage $(0.05 \mathrm{mg} / \mathrm{kg})$ eliminated the scalar property of interval timing, increased the asynchronies and generated a tendency to overestimate of produced intervals. In contrast, the systemic administration of quinpirole did not affect the reaction time in the CT. These findings suggest that a large increase in the activity of the D2 system produce a switch from time-predicting to stimulus-reactive behavior during the rhythmic tapping to a metronome in non-humans primates.

\title{
Keywords
}

Duration, synchrony, behavior, pharmacology, monkey, 100ms

\section{References}

Meck, W. H. (1996). Neuropharmacology of timing and time perception. Brain Res. Cogn. Brain Res., 3, 227-242.

Merchant, H., Luciana, M., Hooper, C., Majestic, S., \& Tuite, P. (2008). Interval timing and Parkinson's disease: Heterogeneity in temporal performance. Exp. Brain Res., 184, 233-248.

\section{Comparing Human and Nonhuman Primate Brain Responses to Auditory Sequences Using EEG}

\author{
Daniel J. Cameron'1, Luis Prado ${ }^{2}$, Jessica A. Grahn ${ }^{3}$ and Hugo Merchant ${ }^{2}$ \\ ${ }^{1}$ Dept. of Neuroscience, Georgetown University, Washington, D.C., USA \\ ${ }^{2}$ Instituto de Neurobiologia, Universidad Nacional Autonoma de Mexico, Queretaro, \\ Mexico \\ ${ }^{3}$ Brain and Mind Institute, Dept. of Psychology, University of Western Ontario, London, \\ Canada
}

Humans have the ability to perceive and move to the 'beat' in musical rhythms. This complex behaviour may be unique among primates, and involves extracting regularities from complex, non-isochronous auditory sequences and synchronizing movements to those regularities. To characterize cross-species differences in neural processing of rhythm and beat perception, we compared brain responses in humans and a macaque monkey using 
electroencephalography (EEG). EEG was recorded while participants listened passively to auditory sequences that consisted of white noise bursts $(65 \mathrm{~ms})$ separated by intervals. Intervals were structured to create 5 types of sequences: i) isochronous (at three rates), ii) random, iii) strongly beat-based, iv) weakly beat-based, or v) non-beat-based. We compared the two species' evoked and induced responses in EEG, at various frequency bands (e.g., beta and gamma) to isochronous and random sequences, and compared neural entrainment at low-frequency $(1-5 \mathrm{~Hz})$ regularities in the structures of non-isochronous sequences (iii-v). Similarities and differences between species' brain responses reveal common and distinct aspects of neural processing of auditory sequences.

Keywords

Rhythm, music, EEG, macaques, humans, 100s of ms-secs

\title{
No Evidence for Improved Attention towards Implicit Temporal Regularities
}

\author{
Atser Damsma ${ }^{1}$, Ritske de Jong ${ }^{1}$, Niels Taatgen ${ }^{2}$ and Hedderik van Rijn ${ }^{1}$ \\ ${ }^{1}$ Department of Psychology, University of Groningen, The Netherlands \\ ${ }^{2}$ Institute of Artificial Intelligence, University of Groningen, The Netherlands
}

In everyday tasks, people pick up and exploit temporal regularities in their environment. To optimize this process, it has been suggested that the attentional system prioritizes information that contains some form of structure. Indeed, Zhao et al. (2013) found that attention was biased towards the location and low-level visual features of shapes that appeared with a regular order but were not relevant for the task at hand. The goal of the current study was to investigate whether this bias also holds for implicit and irrelevant temporal regularities. In two experiments, participants were asked to perform search tasks in which a target appeared in one of four locations. In the first experiment, a sequence of squares was presented in between the search trials in the same four locations. Crucially, in one location, the square appeared with a regular rhythm. In the second experiment, a sequence of colored circles was presented in between the search trials, of which one specific colored circle appeared regularly. We expected that, if attention is automatically biased towards these temporal regularities, reaction times would be faster if the target matches the location or color of the regular stimulus. However, in both experiments, we found no such attentional bias. These results suggest that, although people might keep track of implicit temporal regularities, they do not use this information to guide their attention in the same way as order regularities.

\section{Keywords}

Attention, regularity, prediction, behavior, adult, secs-mins

\section{Reference}

Zhao, J., Al-Aidroos, N., \& Turk-Browne, N. B. (2013). Attention is spontaneously biased towards regularities. Psychol. Sci., 24, 667-677. 


\title{
The Effects of Rhythm-Induced Attention on Perceptual Representation-Mixture-Model Analysis
}

\author{
Asaf Elbaz and Yaffa Yeshurun \\ Psychology Department, University of Haifa, Israel
}

Temporal attention can be entrained exogenously to rhythms. Indeed, faster and more accurate responses were previously found when the target appeared in sync with a preceding rhythm in comparison to when it is out of sync. However, the nature of this rhythm-induced attentional effect is not well understood. To better understand how rhythm-induced attention may affect the quality of the perceptual representation, and specifically to test whether it improves the precision of the target's encoding, we employed a continuous measure of perceived orientation and mixture-model analysis. A trial in our study started with a sequence of beeps that were either separated by a fixed inter-onset interval (IOI; $450 \mathrm{~ms}$ ) in the regular, rhythmic condition or variable IOIs in the irregular condition. A target line followed the sequence. The 'critical' interval between the last beep and the target was chosen randomly from 4 equally likely IOIs (150, 250, 450 and 650 $\mathrm{ms})$. The target was followed by a probe line, and the participants were asked to rotate it to assume the target's orientation. The measure of performance for a given trial was the difference in degrees between the orientation of the target and that reproduced by the participants. The results show a main effect for regularity: the standard deviation (SD) of report errors was significantly smaller when the participants were exposed to regular vs irregular rhythm. Importantly, there was also an interaction between regularity and critical IOI: when the target appeared $450 \mathrm{~ms}$ after the regular rhythm (but not after an irregular rhythm), a smaller SD of report error was found in comparison to other critical intervals. These results suggest that rhythm-induced attention improves the precision of perceptual representation.

Keywords

Rhythms, multisensory, precision, attention, behavior, adult, 100s of ms-secs

\section{Discrimination Accuracy for Visual Rhythms Surprisingly Independent on Several Perceptual Factors}

\author{
Maria Zimmermann and Maksymilian Korczyk \\ Jagiellonian, University, Cracow, Poland
}

In all kinds of timing tasks audition exceeds Vinson. However, the modality- specificity for rhythm perception is particularly puzzling: Accuracy in visual rhythms comparison task 
appears to be lower than it would arise from low visual time resolution itself. What makes visual rhythms processing, especially difficult? We investigate possible explanations. In the first experiment Participants were asked to compare between rhythmical sequences (series of flashes) presented in different time resolution threshold. We found that the accuracy in rhythm perception does not differ significantly across conditions. Crucially, the lack of effect was independent of the overall duration of the sequence as well as number of flashes in a sequence, suggesting that neither time resolution itself, nor visual memory load can be considered as a factor importantly influencing visual rhythms processing. In the second experiment, discrimination accuracy for visual rhythms was investigated for different types of rhythms with either regular or irregular temporal structure without beatinducing qualities. Contrary to what has been shown already (Grahn, 2012) we did not find any significant difference in accuracy for metrical and non metrical rhythms. Finally, we asked if an intensive learning process can have any significant impact on the visual rhythms discrimination. Participants took part in a 10-day training of the visual rhythms comparison task. No main effect of training has been found, the effectiveness of training correlated significantly with the level of musical education. Capacity in visual rhythms processing appears to be highly stable, and independent on several perceptual manipulations. Regarding the effects of music education and recent literature (Barakat, Seitz, \& Shams, 2015), we conclude, that musical experience or auditory training appears to be the crucial factor potentially boosting discrimination, rhythm accuracy in visual rhythms, while strictly visual learning or perceptual visual factors seems to be very little influence.

\title{
Keywords
}

Rhythms, behavior, adult, 100s of ms-secs

\section{References}

Barakat, B., Seitz, A. R., \& Shams, L. (2015). Visual rhythm perception improves through auditory but not visual training. Curr. Biol., 25, R60-R61.

Grahn, J. A. (2012). See what I hear? Beat perception in auditory and visual rhythms. Exp. Brain Res., 220, 51-61.

\section{On-Beat Presentation and Foreperiod Effects in Audio-Visual Rhythmic Entrainment}

\author{
Renaud Brochard and Arnaud Leleu \\ CSGA, Université de Bourgogne Franche-Comté (Dijon) CNRS/INRA/AGROSUP, France
}

In the presence of an auditory background rhythm, visual recognition performance is improved for stimuli that are presented in synchrony with the beat compared to stimuli presented before it. In the present research, we compared after-the-beat presentations of images with on-beat and before-the- beat presentations. Participants $(\mathrm{N}=56)$ were asked to discriminate between meaningful pictures from meaningless textures (their scrambled 
versions) while an irrelevant auditory rhythm was played repetitively. Two types of predictions could be made regarding potential cross-modal effects. On the one hand, with respect to Mari R. Jones' Dynamic Attending Theory, which postulates that attentional resources recursively peak on strong metric positions, reaction times (RTs) should be faster for on-beat visual stimuli compared to presentations before and after the beat. On the other hand, participants could show a "classic" foreperiod effect. In such a case, attention is expected to progressively increase as a function of the wait for the picture to appear. Consequently, RTs should be longest for before-the-beat stimuli, intermediate for on-beat stimuli and shortest for after-the-beat stimuli. Our results showed a clear two-step decrease in RTs with a steeper slope from early off-beat to on-beat presentations and a shallower one from on-beat to late off-beat presentations. Thus, this RT pattern likely reflects the combination of the two forms of temporal attention mentioned above. In a follow-up study, in which different participants $(\mathrm{N}=50)$ performed the same visual task in silence, we only observed a foreperiod effect (of smaller amplitude), confirming the overlap of the two types of temporal attention previously postulated in the audio-visual context. We are currently running EEG studies using a similar procedure. Preliminary results suggest that visual ERPs such as the P1 and the face-selective N170 components might be differentially modulated depending on time position of the pictures relative to the auditory beats.

\title{
Keywords
}

Rhythms, synchrony, multisensory, behavior, adult, 100s of ms-secs

\section{The Fate of Temporal Expectations in Noisy Environments: Robust Extraction of Temporal Regularities Is Limited to Multisensory Events}

\author{
Felix Ball ${ }^{1,2}$, Lara E. Michels ${ }^{1}$ and Toemme Noesselt ${ }^{1,3}$ \\ 1Otto-von-Guericke University Magdeburg, Biological Psychology, Germany \\ ${ }^{2}$ Otto-von-Guericke University Magdeburg, Clinic for Neurology, Germany \\ ${ }^{3}$ Center for Behavioral Brain Science, Magdeburg, Germany
}

Optimization is crucial to cope with the continuous stream of complex multisensory information we perceive every day. There is converging evidence for several optimization mechanisms instrumental in integrating incoming information, among them are multisensory interplay (MSI) and temporal attention (TE). However, whether and how these two mechanisms interact is currently unknown. In a series of psychophysical experiments we tested whether MSI-induced behavioural benefits interact with TE-induced benefits and how these effects are affected by distinct experimental contexts. In all experiments auditory (A) and/or visual (V) stimulus sequences were presented either alone or simultaneously. Participants always discriminated visual and/or auditory frequencies of deviant target stimuli (high/low) within each sequence. Temporal expectation about 
time-of-target-occurrence was manipulated block-wise (early vs. late occurrence). Task difficulty was altered by using speakers ('same location', Exp. 1 \& 3) or headphones ('different location', Exp. $2 \& 4$ ), and by changing the predictability of target modality (predictable: Exp. 1 \& 2, unpredictable: Exp. 3 \& 4). We observed a robust interaction of MSI and TE; with TE effects being enhanced for multisensory relative to unisensory stimulation whereas TE effects for unisensory stimuli even vanished under high spatial uncertainty. Accordingly, computational modelling indicated that TE, target and spatial uncertainty govern multisensory behavioural benefits. Finally, matching expectation information at trial $n-1$ selectively improved performance in the multisensory condition. Together, the pattern of results indicate that multisensory stimulation has a protective and enhancing effect on generating temporal expectations especially in noisy environments, and allows for phasic as well as sustained information extraction to boost performance.

\title{
Keywords
}

Prediction, multisensory, behavior, adult, 100s of ms-secs

\section{Moving and Multisensory Stimulation for Enhancing Visual Rhythm Perception}

\author{
Nadia Paraskevoudi ${ }^{1}$ and Argiro Vatakis ${ }^{1,2}$ \\ ${ }^{1}$ Department of Philosophy and History of Science, University of Athens, Greece \\ ${ }^{2}$ Cognitive Systems Research Institute, Athens, Greece
}

Rhythm perception is affected by the modality in which it is conveyed (Barakat et al., 2015; Grahn, 2012). Despite the dominance of audition in temporal tasks, rhythm may in some cases be mediated by vision as well (Grahn, 2012). Furthermore, auditory rhythm perception can be enhanced by rhythmic visual movement (e.g., human movement) that couples the internal motor system, suggesting a multisensory benefit (Su, 2014). We, thus, aimed to investigate the contribution of multisensory training to rhythm processing. In Experiment 1, we assessed whether multisensory training with animate versus inanimate moving visual stimuli enhances the discrimination ability of visual rhythms utilizing a perceptual learning paradigm. During pre- and post-training, participants completed a rhythm discrimination task, where they had to respond whether the two visual rhythms (i.e., static circles of changing colour) differed or not. Participants were trained with audiovisual metric simple rhythms of two intervals with the visual stimuli being: a) abstractstatic (i.e., static circles; AVstat), b) dynamic-inanimate (i.e., moving bar; Avinan), or c) dynamic-animate (i.e., humanlike point-light figure; AVan) with an explicit beat occurring at regular intervals. Results showed that multisensory training improved post-training performance irrespective of the type of training stimulation. To assess whether this benefit was auditory driven, we introduced a visual- only training with a moving stimulus (Vinan; Exp. 2). Comparisons between AVinan and Vinan showed that despite the absence 
of auditory stimulation in Exp. 2, training with moving stimuli continued to enhance post-training performance. These results, however, could also be contributed to the low complexity of the rhythms presented. Thus, in Experiment 3, we are currently investigating whether multisensory training with moving stimuli (AVinan) benefits processing of both metric simple and complex visual rhythms. Overall, our findings suggest that perceptual learning with visual motion and multisensory stimulation enhances processing of simple visual rhythms.

\title{
Keywords
}

Multisensory, rhythms, behavior, adult, 10s-100s of ms

\section{References}

Barakat, B., Seitz, A.R., \& Shams, L. (2015). Visual rhythm perception improves through auditory but not visual training. Curr. Biol., 25, R60-R61.

Grahn, J.A. (2012). See what I hear? Beat perception in auditory and visual rhythms. Exp. Brain Res., 220, 51-61.

$\mathrm{Su}$, Y.-H. (2014). Visual enhancement of auditory beat perception across auditory interference levels. Brain Cogn., 90, 19-31.

\section{Temporal and Identity Predictions Shape Sensory Responses to Sound in Interaction with Attention}

\author{
Fleur L. Bouwer ${ }^{1,2}$, Heleen A. Slagter ${ }^{1,2}$ and Henkjan Honing ${ }^{1,3}$ \\ ${ }^{1}$ Amsterdam Brain and Cognition, University of Amsterdam, The Netherlands \\ ${ }^{2}$ Department of Psychology, University of Amsterdam, The Netherlands \\ ${ }^{3}$ Institute for Logic, Language and Computation, University of Amsterdam,
} The Netherlands

In the auditory environment, predicting the timing and identity of sounds allows us to focus resources. Such temporal and identity predictions may reduce the computational burden of the brain and optimize the efficiency of the perceptual system. Indeed, auditory evoked potentials (AEPs) are attenuated for sounds with predictable timing or identity (Lange, 2013). Yet, how temporal and identity predictions interact in affecting sensory processing, and whether their influence is mediated by attention, is still matter of debate (Schwartze et al., 2013). Using EEG, we examined the joint influence of temporal and identity predictions on sensory responses to sound, while manipulating task-relevance. We expected predictability of the identity of sounds to attenuate AEPs, regardless of task-relevance, as sound sequences can be learned implicitly. We expected this effect to be magnified by temporal predictability. We constructed sound sequences with a binary structure, in which all sounds in uneven positions were identical (predictable identity), while sounds in even positions were varied (unpredictable identity). Inter-onset intervals 
in the sequences were either regular (predictable timing) or irregular (unpredictable timing). P1 and N1 amplitudes for 34 participants were analyzed using repeated measures ANOVAs with temporal predictability, identity predictability, and task-relevance as withinsubject factors. Both in attended and unattended conditions, we found larger P1 responses to sounds of unpredictable than predictable identity, and this difference was larger for regular than irregular sequences. The same was true for the N1 component, but only when sounds were unattended. With attention, the $\mathrm{N} 1$ was only affected by identity predictions, in line with previous work showing that the attenuating effects of temporal predictability can be counteracted by attention (Todorovic et al., 2015). These findings reveal how temporal and identity predictions jointly influence early sensory processing and suggest that attention may modulate effects of predictions only at later stages of sensory processing.

\section{Keywords}

Prediction, rhythms, music, electrophysiology, adult, 100s of ms-secs

\section{References}

Lange, K. (2013). The ups and downs of temporal orienting: A review of auditory temporal orienting studies and a model associating the heterogeneous findings on the auditory N1 with opposite effects of attention and prediction. Front. Hum. Neurosci., 7, 263.

Schwartze, M., Farrugia, N., \& Kotz, S. A. (2013). Dissociation of formal and temporal predictability in early auditory evoked potentials. Neuropsychologia, 51, 320-325.

Todorovic, A., Schoffelen, J., Ede, F. Van, Maris, E., \& de Lange, F. P. (2015). Temporal expectation and attention jointly modulate auditory oscillatory activity in the beta band. PloS One, 10, e0120288.

\section{Why Can't We Perceive Whisper Speech from a Distance Compared to Normal Speech?}

\section{T. Sushmita, Sudarsana Reddy Kadiri and Suryakanth V. Gangashetty}

Speech Processing Laboratory, International Institute of Information Technology, Hyderabad, India

Whispering is a natural and secondary aspect of speech communication for most people. For some people, whisper may be primary mechanism of communication due to impaired speech production. Unimpaired speakers uses whisper, when communicating sensitive or private information or when speaking in locations such as classrooms, libraries, meetings etc. Whispered speech differ from normal voiced speech in terms of vowel duration, spectral tilt etc., with similar formants (vocal tract shape) information (Su et al., 2016). Existing studies shown that performance of whisper speech recognition reduces due to absence of pitch information. However, there remains a lack of good evidence for the whisper speech comprehension from a distance. In this study, we hypothesis that, this is mainly due to 
variations in the timing instants of the abrupt closure of vocal folds during speech production. In general, this abrupt closure reflects as an impulse-like discontinuity in the speech and it happens approximately for every $10 \mathrm{~ms}$ in male and $5 \mathrm{~ms}$ in female speakers (Murty \& Yegnanarayana, 2008). The hypothesis is validated through analysis by synthesis (AbS) experiments using linear prediction (LP) analysis. LP analysis decomposes the signal into LP residual and LP coefficients, where LP residual corresponds to the excitation source which consists of timing instants of abrupt closure of vocal folds and LP coefficients corresponds to vocal tract information. Initially, we investigated the LP residuals of normal and whisper speech and then $\mathrm{AbS}$ experiments are conducted by inserting and removing the timing instants of abrupt closure of the vocal folds in normal and whisper speech. From the experimental analysis, it was found that due to absence of the timing instants of vocal fold closure in whisper, we are not able to get the message by listening from a distance. This is also true for normal speech, if we remove the timing instants of vocal fold closure. The neural mechanism of human beings seem to have the ability of selectively processing these timing instants (Abrams et al., 2017), which is evident from Figures 8, 9 and 11 where timing instants of vocal fold closure are well matching with the multi-unit activities). This ability of responding to microlevel timing events may be responsible for extracting robust and reliable speech information even under severe degradations such as noise, reverberation and presence of multiple speakers. The analysis and results of the study will be demonstrated at the conference.

\title{
References
}

Murty, K. S. R., \& Yegnanarayana, B. (2008). Epoch extraction from speech signals. IEEE Transactions on Audio, Speech, and Language Processing, 16, 1602-1613.

Su, Q., Galvin, J. J., Zhang, G., Li, Y., \& Fu, Q.-J. (2016). Effects of within-talker variability on speech intelligibility in Mandarin-speaking adult and pediatric cochlear implant patients. Trends Hear., 20.

\section{Dynamic Prosodic Features in Bipolar Disorder: How Shifting Vocal Patterns in Verbal Fluency Tasks Can Aid the Detection of Mixed Symptoms}

\author{
Luisa Weiner ${ }^{12}$, Nicola Vanello ${ }^{3,4}$, Andrea Guidi ${ }^{3,4}$, \\ Enzo Pasquale Scilingo ${ }^{3,4}$, Nadège Doignon-Camus ${ }^{1}$, \\ Gilles Bertschy ${ }^{1,2}$ and Anne Giersch ${ }^{1}$ \\ ${ }^{1}$ INSERM 1114, Strasbourg, France \\ ${ }^{2}$ University Hospital of Strasbourg, France \\ ${ }^{3}$ Dipartimento di Ingegneria dell'Informazione, University of Pisa, Pisa, Italy \\ ${ }^{4}$ Research Center "E. Piaggio", University of Pisa, Pisa, Italy
}


Bipolar disorder is characterized by episodes of mania, depression, and mixed episodes whereby depressive and manic symptoms may co-occur. Although patients frequently experience mixed episodes, the clinical boundaries between mixed depression and typical depression, and mixed mania and typical mania, are still a matter of debate. Studies have shown that speech parameters could be used to obtain relevant information regarding patients' mood states. One such parameter is the fundamental frequency (F0), i.e., the lowest frequency of a periodic waveform. F0 contour in emotional speech perception and, more specifically, features related to the temporal prosodic dynamics have been found to provide more information than global, static prosodic features. The aim of this work is to investigate whether F0 dynamics are able to distinguish mixed states from typical depression and mania in patients with bipolar disorder. Nineteen patients in manic episode, 8 patients with mixed mania, 17 patients with mixed depression, and 12 patients with a depression participated in this study. Speech samples were acquired via nine verbal fluency tasks. Participants were asked to produce as many words as possible during 120s. Voiced segments were estimated so that single words could be highlighted. Prosodic features, derived from Taylor's tilt intonational model, were estimated on each voiced segment. Specifically, features were related to the shape of intonation and its temporal dynamics. Preliminary findings show that leave one out classification could successfully discriminate between mixed mania and mania (accuracy $88.8 \%$, specificity $94.4 \%$, sensitivity $77.7 \%$, F1 score $82 \%$ ), and mixed depression and depression (accuracy $72 \%$, specificity $70 \%$, sensitivity $74 \%$, F1 score $78 \%$ ). These results suggest that discrete prosodic modulations over time might be informative regarding the presence of mixed symptoms. Future analyses will focus on other time-related parameters, such as inter-words pauses, and their relationship to the speed of thoughts in bipolar disorder.

Keywords

Adults, bipolar disorder, language, neuropsychology, 100s of ms-secs

\title{
Rhythm, Harmony and Musical Training Interact to Affect the Sensation of Groove as well as Activation in Motor and Reward Areas
}

\author{
Tomas Matthews ${ }^{1}$, Maria Witek ${ }^{2,3}$, Ole Heggli² ${ }^{2}$, Torben Lund ${ }^{2}$, \\ Virginia Penhune ${ }^{1}$ and Peter Vuust ${ }^{2,3}$ \\ ${ }^{1}$ Concordia University, Canada \\ ${ }^{2}$ Aarhus University, Denmark \\ ${ }^{3}$ Royal Academy of Music, Denmark
}

Groove is defined as 'a pleasurable drive towards body-movement in response to rhythmically entraining music' (Vuust \& Witek, 2014). Recent work has shown that there is an inverted U-shaped relationship between rhythmic complexity, the desire to move and 
evoked pleasure (Witek et al., 2014). However, the role of factors beyond rhythmic complexity in contributing to the sensation of groove have yet to be clarified. Although the sensation of groove is thought to result from interactions between motor and reward systems, this interaction has yet to be shown explicitly. Two studies were implemented to investigate the neural basis of groove-related pleasure and determine whether harmony contributes to our enjoyment of groove music. In an online study, ratings of pleasure and wanting to move were collected for musical patterns that varied in both harmonic and rhythmic complexity. Results showed an inverted U-relationship between subjective ratings and rhythmic complexity, but not harmonic complexity. Although rhythmic complexity seems to dominate, harmonic complexity did affect the desire to move as well as pleasure. In another study, musicians and non-musicians rated a subset of the musical patterns used in the online study while undergoing functional magnetic resonance imaging (fMRI) and facial electromyography, in two separate sessions. Rating results replicated those of the online study. fMRI results showed that dorsal basal ganglia activity tracked rhythm complexity while ventral basal ganglia activity matched the pattern of subjective ratings, showing an interaction between harmonic and rhythmic complexity. Additionally, musicians showed greater motor activity while listening compared to non-musicians. These results show that harmony contributes to the sensation of groove in addition to rhythm. Furthermore, these results show that musical training may lead to a more actionbased processing of music and supports the putative link between motor and reward processes in generating the sensation of groove.

\section{Keywords}

Rhythms, music, MRI, behavior, adult, 100s of ms-secs

\section{References}

Vuust, P., \& Witek, M. A. G. (2014). Rhythmic complexity and predictive coding: A novel approach to modeling rhythm and meter perception in music. Front. Psychol., 5, 1-14.

Witek, M. a G., Clarke, E. F., Wallentin, M., Kringelbach, M. L., \& Vuust, P. (2014). Syncopation, body-movement and pleasure in groove music. PloS One, 9, e94446.

\section{Drum-Playing Modulates the Post-Auricular Muscle Response}

\section{Zoe Swann and Patrick Simen}

Neuroscience Department, Oberlin College, USA

The post auricular muscle, located behind the ear above the mastoid bone, is known to produce a brief, involuntary reflex roughly 10 milliseconds after high-frequency sounds in many (but not all) human participants. The electrical signature of this vestigial response, the post-auricular muscle response (PAMR), is a muscle action potential observable in 
electrodes placed above the mastoids (O'Beirne \& Patuzzi, 1999). We found that the PAMR was bilaterally modulated by task goal for equivalent sound stimuli (snare drum sounds). We sought to investigate the brain basis of inter-beat interval timing in rhythmic behavior. We used electroencephalography (EEG) to monitor neural and muscular signals while human participants actively produced sequences of drumbeats, with passive listening and non-rhythmic drumming tasks as control conditions. In each condition, participants produced or listened to rhythmic or non-rhythmic patterns of snare drumbeats at 70-100 beats per minute for two minutes. Resulting PAMR amplitudes, surprisingly, ranged from 5-200 microvolts $(\mu \mathrm{V})$ in active drumming, versus 3-70 $\mu \mathrm{V}$ in passive listening. Within participants, active drumming produced a dramatically larger average PAMR than passive listening, and rhythmic drumming/listening produced larger average PAMRs than corresponding non-rhythmic conditions. We further observed a distinct latency difference relative to stimulus time between active and passive trials, with active responses occurring approximately 8 milliseconds ( $\mathrm{ms}$ ) after the snare sound, and passive responses at approximately $12 \mathrm{~ms}$ after the sound. The latency of the PAMR suggests it is mediated by only a few synaptic connections between cochlea and motor neurons, yet modulation by task goal suggests a top- down effect in which attention to rhythmic production can functionally enhance this connectivity. PAMR magnitude and latency may therefore serve as useful tools for investigating the brain basis of timed, rhythmic behavior.

\title{
Keywords
}

Rhythms, music, electrophysiology, adult, 10s-100s of ms

\section{Reference}

O'Beirne, G. A., \& Patuzzi, R. B. (1999). Basic properties of the sound-evoked post-auricular muscle response (PAMR). Hear. Res., 138, 115-132.

\section{Mapping the Beat Bin: Effects of Rise Rime, Duration and Frequency Range on the Perceived Timing (P-Center) of Musical Sounds}

\author{
Anne Danielsen ${ }^{1}$, Justin London ${ }^{2}$ and Kristian Nymoen ${ }^{1}$ \\ ${ }^{1}$ University of Oslo, Norway \\ ${ }^{2}$ Carleton College, USA
}

The perceived temporal placement of a musical sound (i.e., its P-center) has received little attention in music research, but previous research has shown that the P-center is not equal to the sound's perceived onset (Wright, 2008), and is probably a flexible "bin" rather than a point in time (Danielsen et al., 2015). In this paper, we report on several experiments aimed at investigating the effect of rise time, frequency range and sustained versus decaying amplitude on the P-center of musical sounds. Stimuli consisted of eight 
recorded sounds of acoustical and electronic instruments with balanced combinations of features: short vs. long rise time, high vs. low frequency range, and sustained versus decaying amplitude (duration). All stimuli were presented at an ISI of $600 \mathrm{~ms}$ (100 bpm). The task was to align a click with the stimuli sound. The participants $(\mathrm{N}=20)$ were musically trained and performed the task $4 \times$ per stimulus. Mean click alignment varied from 1-23 ms after stimulus onset. The standard deviation (SD) from average click locations was $7-21 \mathrm{~ms}$. Repeated Measures ANOVAs show a main effect of rise time $(\mathrm{F}(1,19)=42.428$, $\mathrm{p}<0.001 ; \eta 2=0.170)$, duration $(\mathrm{F}(1,19)=9.702, \mathrm{p}=0.01 ; \eta 2=0.050)$ and frequency $(\mathrm{F}(1,19)=12.018, \mathrm{p}<0.01 ; \eta 2=0.031)$ on click alignment position. We also found a main effect of rise time $(\mathrm{F}(1,19)=37.026, \mathrm{p}<0.001 ; \eta 2=0.049)$ and duration $(\mathrm{F}(1,19)=$ 10.733, $\mathrm{p}<0.01 ; \eta 2=0.070)$ for the SD. The results show that slow rise times lead to later P-centers for a range of musical sounds, in accord with previous research into speech and artificial sounds (Villing, 2010). Interestingly, sustained amplitude and low frequency seem to have the same effect. Slow rise time and sustained amplitude also yield higher standard deviation and may point towards the timing window, or beat bin, being wider for these sounds. Trials are ongoing with additional stimuli; results of those experiments may also be reported.

\title{
Keywords
}

Rhythms, synchrony, behavior, adult, 10s-100s of ms

\section{References}

Danielsen, A., Haugen, M. R., \& Jensenius, A. R. (2015). Moving to the beat: Studying entrainment to micro-rhythmic changes in pulse by motion capture. Timing Time Percept., 3, 133-154.

Villing, R. C. (2010). Hearing the moment: Measures and models of the perceptual centre. Phd Thesis, Department of Electronic Engineering, National University of Ireland Maynooth.

Wright, M. J. (2008). The shape of an instant: Measuring and modeling perceptual attack time with probability density functions. Phd Thesis, Stanford.

\section{Exploring the Neural Basis of Musical Timing not Related to a Regular Beat}

\author{
Erik Christensen
}

Aalborg University, Denmark

Investigations of music not related to a regular beat may yield important contributions to understanding the neural basis of timing. Various types of music from different cultures indicate that the mind can organize temporal patterns without reference to a beat (Patel, 2008; Stevens \& Byron, 2016). Unpulsed music may be related to movements and gestures, graphics, speech, birdsongs, environmental sounds, artificial sounds, and temporal 
patterns created by composers. Indian ragas, which juxtapose music with and without a pulse, can provide ample material for investigations. I propose to conduct an fMRI study based on short excerpts of ragas, exploring the neural structures recruited by listening to 1) the unmetered part of the raga, 2) the metered part, and 3) the drone alone, by adapting the sparse sampling method developed by Teki et al. (2011). A group of Indian participants familiar with listening to ragas can be compared with a group of different participants. It is my proposition that music can evoke embodied sensations of time within three categories: time related to movements and events, time related to a regular pulse, and time related to apparent stasis. Further investigations may include music composed by Brian Eno, Morton Feldman, György Ligeti, Olivier Messiaen and Iannis Xenakis (Christensen, 2012).

\title{
References
}

Christensen, E. (2012). Music listening, music therapy, phenomenology and neuroscience.

PhD thesis, Aalborg University. http://www.mt-phd.aau.dk/phd-theses/.

Patel, A. D. (2008). Music, language, and the brain. Oxford University Press.

Stevens, C. J., \& Byron, T. (2016). Universals in music processing. The Oxford Handbook of Music Psychology, 19-30.

Teki, S. et al. (2011). Distinct neural substrates of duration-based and beat-based auditory timing.J. Neurosci., 31, 3805-3812.

\section{Rhythmic Perceptual Priors Revealed Cross-Culturally by Iterated Reproduction}

\author{
Nori Jacoby ${ }^{1,2}$ and Josh McDermott ${ }^{2}$ \\ ${ }^{1}$ The Center for Science and Society, Columbia University, New York, NY, USA \\ ${ }^{2}$ Department of Brain and Cognitive Sciences, Massachusetts Institute of Technology, \\ Cambridge, MA, USA
}

Probability distributions over external states (priors) are essential to the interpretation of sensory signals (Weiss et al., 2002). We propose a novel method to characterize perceptual priors and apply it to the study of simple rhythms. To characterize priors for this domain we developed a method based on iterated reproduction of random temporal sequences (Jacoby \& McDermott, 2017). Listeners were asked to reproduce random "seed" rhythms; their reproductions were fed back as the stimulus, and over time became dominated by internal biases, such that the prior could be estimated by applying the procedure multiple times. Using the method, we measured listeners' priors over the entire space of two- and three-interval rhythms, examining Westerners with different levels of musical expertise as well as members of the Tsimane, a native Amazonian society with very limited exposure to Western music. We found that priors in Westerners showed peaks at rhythms with simple integer ratios, but only those that are prevalent in Western music. Priors were similar for musicians and nonmusicians, suggesting that they are shaped primarily by passive 
exposure to the music of a culture. Priors in Amazonians also exhibited modes at integer ratios, but were otherwise qualitatively different from priors in Westerners, in ways that are consistent with the structures prevalent in their music. The results were similar for several different modes of reproduction (e.g., finger tapping and rhythmic vocalization of a repeated syllable), but did not extend to the reproduction of spoken phrases, indicating that integer ratio priors are at least somewhat specific to music. We consider the claim that rhythm perception exhibits universal cognitive constraints (Ravignani et al., 2017) and argue that our results indicate that any such constraints are strongly modulated by culturally dependent musical exposure.

\title{
Keywords
}

Rhythm, music, beat, comparative, cross-cultural

\section{References}

Jacoby, N., \& McDermott, J. H. (2017). Integer ratio priors on musical rhythm revealed cross-culturally by iterated reproduction. Curr. Biol.

Ravignani, A., Delgado, T., \& Kirby, S. (2016). Musical evolution in the lab exhibits rhythmic universals. Nat. Hum. Behav., 1, 0007.

Weiss, Y., Simoncelli, E. P., \& Adelson, E. H. (2002). Motion illusions as optimal percepts. Nat. Neurosci., 5, 598-604.

\section{In Search of Differential Enculturation and Entrainment Effects in Rhythmic Grouping}

\author{
Ece Kaya and Esra Mungan \\ CEA, DSV/I2BM, NeuroSpin; INSERM, U992, Cognitive Neuroimaging Unit; \\ Univ Paris-Sud, F-91191 Gif/Yvette, France
}

The aim of this study was to see if rhythmic grouping is influenced by enculturation or training. Participants who are encultured or entrained to the complex meters may reveal this by changing sensitivities to durational deviations in different sections of pulsed sequences. In our experiment, we used a gap-detection paradigm in which listeners had to detect slight tempo deviations within otherwise regularly pulsed sequences. We were interested in whether a pre-existing subgrouping of a regularly pulsed sequence (e.g., $3+2$ or $2+3$ for a $5 / 8$ meter or $3+3$ for a $6 / 8$ meter) could lead to lower detection rates of duration deviations occurring at rather than within sub-grouping boundaries. Thirty-six Turkish nonmusicians listened to a mixed list of $5 / 8$ and $6 / 8$ pulse sequences, each of which contained a single $\pm 0,20,25,30,35,40$, or 45 ms tempo deviation. Pulses were played in bongo timbre at $120 \mathrm{bpm}$. Critically, unlike XXXOOO type gap detection paradigms, our sequences were identical in terms of pitch, accent or the like. Listeners had to press an "equal", "early" or "late" button to report whether or what kind of a deviation they 
detected. Results showed that participants were less sensitive to deviations that occurred in the beginning or end (e.g., X_XXXX or XXXX_X) and more sensitive to deviations at potential subgrouping boundaries (e. g., XX_XXX or XXX_XX). Yet, nonmusicians might not be chunking at all when hearing psychoacoustically identical pulse sequences. We are currently collecting data from Turkish musicians (half of them percussionists highly trained in complex meters) and Western listeners encultured into duple and triple meters only. We also plan to extend our findings to frequently used meters in Turkish music such as $7 / 8$ and $9 / 8$. Results will be discussed with respect to local and global influences in rhythmic grouping.

\title{
Keywords
}

Prediction, rhythms, behavior, adult, 10s-100s of ms

\section{Collective Metacognition in Improvised Music Ensemble}

\author{
Pedro Barreiros ${ }^{1}$ and Renato Soeiro ${ }^{2}$ \\ ${ }^{1}$ Universidade de Aveiro - DECA, Portugal \\ ${ }^{2}$ INESC TEC, Portugal
}

In ensemble music created in real time, the proportion of improvisation is indexed to aesthetics (Pressing, 1984). However, all music interaction relies on synchronisation/ entrainment and, according to Repp and Keller (2004), adaptation to tempo changes rests on two processes: period and phase correction. Thus, a musician develops mainly two cognitive behaviours: a sequential memory, and a library with audio symbols. With improvisation the musician has the possibility of using this library to create new content "driving" or to change the context "backing" (soloist/ rhythmic and harmonic support). The goal of this work is creating improvisational contexts to study and develop these two roles. There are 9 participants forming 16 trios and 33 consecutive weekly sessions where one trio (voice, guitar, double-bass) performs 21 songs. In each song the drive/ backing roles are distributed over 5 distinct moments with a written plan for each musician to play both roles. A common digital music sheet is used to continuously present a visual reference with the dynamic localisation of the harmonic progression and tempo reference at measure resolution. Every session is recorded with video and multitrack audio, including the tempo reference. The collective musical metacognition is developed through this bi-modal entrainment context: musicians and digital music sheet. "Driving" requires a perception of the "backing" rhythmic flexibility, and "backing" requires an understanding of the "driving" music narrative. The visual reference information allows evaluating the collective tempo deviation.

\section{Keywords}

Music, behaviour, adult, 10s-100s of ms, entrainment 


\title{
References
}

Pressing, J. (1984). Cognitive processes in improvisation. Adv. Psychol., 19, 345-363.

Repp, B. H., \& Keller, P. E. (2004). Adaptation to tempo changes in sensorimotor synchronisation: Effects of intention, attention, and awareness. Q.J. Exp. Psychol., 57A, 499-521.

\section{Human Speed in Music}

\author{
Ned McGowan \\ Leiden University, The Netherlands
}

In this presentation, the experience of speed in music is dissected. Organic to all cultures, music is likely an emergent property of our biological existence, a manifestation of who we are, how we think and how we feel (Craig, 2009). Organic to music, on the other hand, is time, because without time there is no music. Nonetheless, the phenomenon of time with its broad implications in the sciences tells little about our experiences. The concept of speed, though, is full of enlightening character. Take some common terms to describe tempo in music: largo (broadly), adagio (slow and stately), allegro (fast, quickly and bright), vivacissimo (very fast and lively). Speed is relational and reveals how we think and feel. It is innately human. Short musical recordings explore the full range of musical speed, from sounds placed into stasis to entire symphonies repeated at a frequency beyond the range of human detection, demonstrating how the identity of music undergoes various metamorphoses as the temporal scale is altered. Temporal resolution in music is also explored, according to one of Olivier Messiaen's three laws of experienced duration: “... the more events in the present, the shorter our experience of duration for that moment in the present; the fewer events, the longer our experience of duration" (Delaere, 2009). Based on artistic and pedagogic experience, the arguments consider the embodiment of rhythm and duration as experienced by practicing musicians. Questions such as whether we can think in time and the relationship between emotions and speed are discussed from the musical perspective.

\section{Keywords}

Duration, music, resolution, neuropsychology, adult, 100s of ms-secs

\section{References}

Craig, A. (2009). Emotional moments across time: A possible neural basis for time perception in the anterior insula. Philos. Trans. R. Soc. Lond., B, Biol. Sci., 364, 1933-1942.

Delaere, M. (2009). Tempo, metre, rhythm. Time in twentieth-century music. Unfolding time: studies in temporality in twentieth-century music, 31. D. Crispin, (Ed.). Leuven: Leuven University Press. 


\title{
Is this Music, the Extraordinary Nature of a Single Drumming Bout Compared to Ordinary Noise Making in Chimpanzees
}

\author{
V. Dufour ${ }^{1}$, C. Pasquaretta ${ }^{1}$ and E. H. M. Sterck ${ }^{2}$ \\ ${ }^{1}$ IPHC, UMR7178, CNRS, University of Strasbourg, France \\ ${ }^{2}$ University of Utrecht, BPRC, The Netherlands
}

Here, I will present the unusual characteristics of the drumming performance of a chimpanzee named Barney. His sound production, several sequences of repeated drumming on an up-turned plastic barrel, shared several features of typical human musical drumming: it was rhythmical, decontextualized, and well controlled by the chimpanzee (Dufour et al., 2015). This type of performance raises questions about the origins of our musicality. Following this work we also recorded all events of sound production with objects that spontaneously occurred in Barney's colony. First we looked at the frequency and duration of sound making. We examined effects of context of sound making, the sex of the producer, as well as the medium and the degree of elaboration of the technique used. Then we filmed as many events as possible to increase our chances of recording sequences that would be musically similar to Barney's performance in the original study. Interestingly, properties of sound productions differed across contexts, sex, and techniques. We also filmed several long productions that were rhythmically interesting. However, none fully met the criteria of Barney's production in terms of musicality (Dufour et al., 2017). Nevertheless, the high frequency of occurrence of sound making with objects in the observed colony may have concurred to create favorable conditions leading to this "once in a lifetime" music making by Barney.

\section{Keywords}

Rhythm, music, behaviour, Chimpanzees, 100s of ms-secs, drumming

\section{References}

Dufour, V., Pasquaretta, C., Gayet, P., \& Sterck, E. H. M. (2017). The extraordinary nature of Barney's drumming: A complementary study of ordinary noise making in Chimpanzees. Front. Neurosci., 11, 2.

Dufour, V., Poulin, N., Charlotte Curé, \& Sterck, E. H. M. (2015). Chimpanzee drumming: A spontaneous performance with characteristics of human musical drumming. Sci. Rep., 5, 11320. 